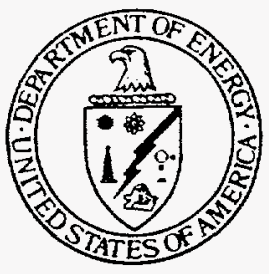

Department of Energy

Oak Ridge Operations

Weidon Spring Site

Remedial Action Project Office

7295 Highway 94 South

St. Charles, Missouri 63304
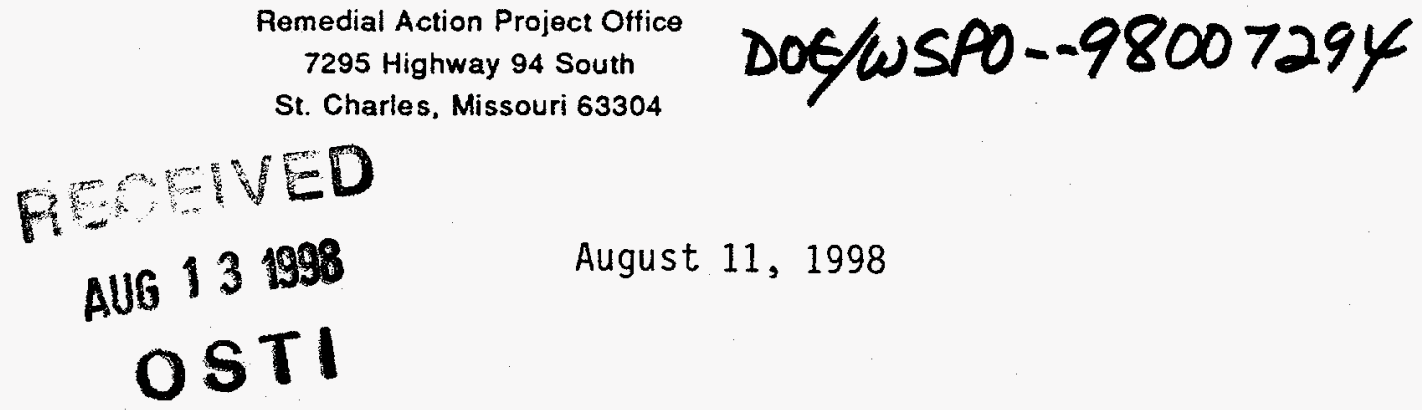

August 11, 1998

Distribution:

\title{
QUARTERLY ENVIRONMENTAL DATA SUMMARY FOR SECOND QUARTER 1998
}

In support of the Weldon Spring Site Remedial Action Project Federal Facilities Agreement, a copy of the Quarterly Environmental Data Summary (QEDS) for the second quarter of 1998 is enclosed.

The data presented in this letter and attachment constitutes the QEDS. The data were received from the contract laboratories, verified by the Weldon Spring Site verification group and, except for air monitoring data and site KPA generated data (uranium analyses), merged into the database during the second quarter of 1998. Air monitoring data presented are the most recent complete sets of quarterly data. Air data are not stored in the database and KPA data are not merged into the regular database.

Significant data, defined as data values that have exceeded defined above normal level 2 values as described below, are discussed in this letter for Environmental Monitoring Plan (EMP) generated data. Above normal level 2 values are based, in ES\&H procedures, on historical high values, DOE Derived Concentration Guides (DCGs), NPDES limits and other guidelines. The procedures also establish actions to be taken in the event that above normal data occur.

All data received and verified during the second quarter were within a permissible range of variability, except for those detailed below. Above normal occurrences are cited for groundwater, air, and NPDES data. There were no above normal occurrences for springs or surface water. The following discussion offers a brief summary of the data merged during the second quarter that exceeded the above normal criteria and updates previously reported above normal data. The attached tables present the most recent data for air and the data merged into the database during the second quarter 1998 for groundwater, NPDES, surface water, and springs.

Graphs showing concentrations of selected contaminants of concern at some of the critical locations have also been included in this QEDS. The graphs are discussed in the separate sections.

\section{NPDES}
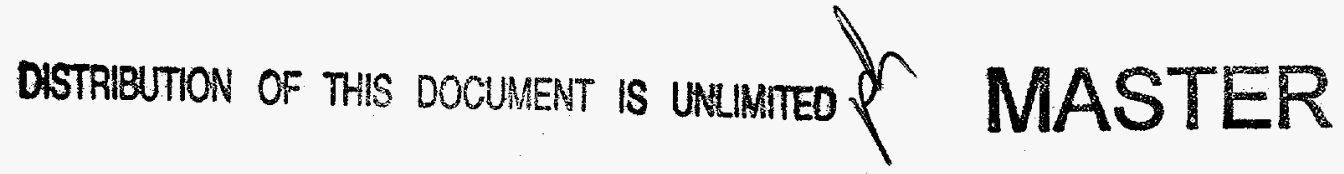

The uranium concentrations at the three major NPDES outfalls are graphed for April 1997 to June 1998. The graphs are located at the beginning of the NPDES tables. For comparison, the annual average for 1997 is also graphed. The derived concentration guide (DCG) of $600 \mathrm{pCi} / 1$ for uranium may also be used for comparison. The DCG is the concentration of a radionuclide in air or water that, under conditions of continuous exposure for one year by one exposure mode (i.e., ingestion of water), would result in an effective dose equivalent of $100 \mathrm{mrem}$. A graph of historical annual averages for the three outfalls is also attached.

Outfall NP-0002 was below the 1997 annual average for uranium (14 pCi/l) for each of the three months of the quarter. Most of the NP-0002 watershed has been remediated and uranium levels are expected to stay well below the pre-remediation levels. 


\section{DISCLAIMER}

This report was prepared as an account of work sponsored by an agency of the United States Government. Neither the United States Government nor any agency thereof, nor any of their employees, makes any warranty, express or implied, or assumes any legal liability or responsibility for the accuracy, completeness, or usefulness of any information, apparatus, product, or process disclosed, or represents that its use would not infringe privately owned rights. Reference herein to any specific commercial product, process, or service by trade name, trademark, manufacturer, or otherwise does not necessarily constitute or imply its endorsement, recommendation, or favoring by the United States Government or any agency thereof. The views and opinions of authors expressed herein do not necessarily state or reflect those of the United States Government or any agency thereof. 


\section{DISCLAIMER}

Portions of this document may be illegible in electronic image products. Images are produced from the best available original document. 
The uranium levels at NP-0003 were well below the 1997 annual average $(143 \mathrm{pCi} / \mathrm{l})$ for the three months of the quarter. While most of the Outfall NP-0003 watershed has been remediated, it contains Ash Pond where contaminated soils, concrete, etc., are being stored. This has probably contributed to the higher levels at NP-0003 for 1997 and early 1998 . Water is released from the Ash Pond area only if it is less than $600 \mathrm{pCi} / \mathrm{l}$; however it may still be higher than the previous Outfall NP-0003 annual average, which could cause an increase in the current annual average. The volume of runoff from Ash Pond is low in comparison to the remainder of the watershed. An upward trend for uranium is not expected because the surface area exposed to storm water is not expected to increase. Stabilization of soil piles stored in the Ash Pond area has helped reduce uranium levels in the storm water runoff. Material is now being removed for placement in the cell, which may cause uranium levels to fluctuate slightly.

Outfall NP-0004 was added to the NPDES permit during May of 1998 . The watershed that flows to the outfall includes the remediated section of Pit 4 . There was one discharge from the pit area during June. The uranium level was $4.7 \mathrm{pCi} / 1$, which is well below the DCG. Because NP-0004 is a minor outfall, it is not graphed.

Outfall NP-0005 was below the 1997 annual average for uranium (19 pCi/l) for the three months of the quarter. A large portion of the NP-0005 watershed has been remediated and uranium levels are expected to remain well below pre-remediation levels.

Outfall NP-0010, the CMSA sedimentation basin outfall, remained below the 1997 annual average $(2.7 \mathrm{pCi} / 1)$ for April for uranium. For May and June, uranium was slightly greater than the 1997 annual average but still much less than the DCG of $600 \mathrm{pCi} / 1$. The area has been remediated and uranium levels are expected to remain low, but the use of a small area for vehicle maintenance may increase uranium levels slightly. Because NP-0010 is a minor outfall, it is not graphed.

The above normal data merged during the second quarter and any above normal data that were not resolved in previous QEDS are discussed below.

\section{NP-0003-060498}

On June 4, 1998, a sample was collected at Outfall NP-0003 that had a settleable solids level of $125 \mathrm{ml} / 1 / \mathrm{hr}$. The limit for settleable solids is $1 \mathrm{ml} / 1 / \mathrm{hr}$. Over 1 inch of rain had fallen, starting the night before, and it was raining during the sample event. Work taking place on the clean-fill dike east of the diversion channel was determined to be the source of the sediment. Additional rock check dams were placed upstream of the sedimentation basin and additional erosion control measures will be investigated for implementation after the area dries. Maintenance measures, including sediment removal, for the sedimentation basin will also be investigated. A subsequent sample collected on June 9, 1998, was in compliance with settleable solids at a level of less than $0.1 \mathrm{ml} / 1 / \mathrm{hr}$.

This same sample also had a chromium level of $111 \mu \mathrm{g} / 1$. NPDES permit MO-0107701 special condition C.2. specifies that we notify the MDNR if any toxic pollutants exceed specified levels. In the case of chromium, that level is $100 \mu \mathrm{g} / 1$; therefore, this chromium result required a Level 2 notification Other metals were also elevated but not above the notification levels. Although soil and debris are being removed from the Ash Pond area, based on the analytical results and 
visual observation, we believe that the elevated chromium levels are related to the elevated solids levels. Actions were taken as noted above to reduce solids levels. Metals will also be analyzed in samples from NP-0003, Ash Pond, and diversion channel water in July storm water samples to help determine the source.

NP-0006-061698

A compliance sample for sewage treatment plant effluent was collected on July 16,1998 . The fecal coliform result was 1,200 colonies per $100 \mathrm{ml}$ with a duplicate result of 1,160 colonies per $100 \mathrm{ml}$. The permitted daily maximum is 1000 colonies per $100 \mathrm{ml}$.

All other parameters were in compliance and the total residual chlorine (TRC) level was 0.06 $\mathrm{mg} / \mathrm{l}$. Discussions with the Construction Engineer (CE) who oversees operation of the plant revealed that the plant was recovering from a minor upset where the $\mathrm{pH}$ may not have been at an optimum level for chlorination effectiveness. This information, along with the relatively low TRC level, suggests that the upset is the cause of the elevated level. The treatment plant is now operating properly and a sample will be collected during the week of August 10,1998, for fecal coliform analysis to ensure that proper disinfection is taking place. No degradation of the receiving stream was noted.

\section{GROUNDWATER}

\section{Chemical Plant}

Site Water Treatment Plant and Temporary Storage Area

The unfiltered samples from two monitoring locations continued to exceed baselines (chromium and lead at MW-2040, and lead at MW-2043); however, concentrations of metals in filtered samples did not exceed baseline values. The samples were filtered because baseline values were established using filtered sample data. The results of these comparisons, summarized below, indicate that there is no impact (due to elevated metals levels) on ground water at the site water treatment plant and temporary storage area.

\section{- Sample GW-2040-Q198}

The unfiltered sample for the first quarter of 1998 for this location was reported with concentrations of chromium (30.5 ug/l) and lead $(7.7 \mathrm{ug} / 1)$ above baseline values $(14.1 \mathrm{ug} / 1$ and $3.3 \mathrm{ug} / 1$, respectively); however, the concentrations for the filtered duplicate of this sample did not exceed the baseline values for either metal. The reported concentrations were $9.2 \mathrm{ug} / \mathrm{l}$ for chromium and no detection $(<0.90 \mathrm{ug} / 1)$ for lead.

\section{- Sample GW-2043}

The first quarter 1998 unfiltered sample was reported with a lead concentration $(3.2 \mathrm{ug} / \mathrm{l})$ exceeding baseline $(2.81 \mathrm{ug} / \mathrm{l})$ for this location. The filtered duplicate had no detection for lead $(<0.90 \mathrm{ug} / 1)$. 
VOC Monitoring

- Samples GW-2037-1197, GW-2037-1297, GW-2037-B197, GW-2038-1197, GW-20381297, GW-2038-B198, GW-3025-1197, GW-3025-1297, GW-3025-B198, MW-S004B198, MW-S021-1197, MW-S021-1297, and MW-S021-B198.

Monitoring for Volatile Organic Compounds (VOCs), specifically trichloroethene(TCE), south and west of Raffinate Pits 3 and 4 at WSSRAP locations and Department of Army locations continued through the second quarter of 1998 . Bimonthly monitoring for the same locations began during the first quarter of 1998 . No significant TCE concentration increases were observed during this quarter. Location MW-2038 continues to show steady, significant decreases in TCE concentrations (from above $1000 \mathrm{ug} / \mathrm{l}$ in late 1996 down to $230 \mathrm{ug} / 1$ in second quarter, 1998).

This indirectly suggests that the source is in the vicinity of the southern portion of Raffinate Pit 3 and that, since late 1995 or early 1996, the source is contributing to the groundwater contamination to a lesser extent.

Enhanced Raffinate Pit Area Monitoring

Enhanced Raffinate Pit area groundwater monitoring at selected locations in the southern and western portions of the site is intended to provide increased capability of detecting impacts on groundwater from disturbance of raffinate pit sludge and soil during ongoing remediation efforts. The monitoring includes monthly sampling for 11 groundwater monitoring locations (MW-2037, MW-2038, MW-2039, MW-3003, MW-3023, MW-3024, MW-3025, MW-3027, MW-4001, MW-4002, and MW-4006). The analytical parameters for the 11 locations include nitrate, sulfate, metals (Toxicity List), and total uranium (on-site KPA). No abnormal values were reported during the second quarter 1998 monitoring period.

\section{Disposal Cell Detection Monitoring}

Groundwater sampling for the first quarter 1998 Disposal Cell Detection Monitoring System (MW-2032, MW-2045, MW-2046, MW-2047, and MW-2048) was completed in January 1998. The analytical results from this event were within the expected ranges (based upon the previous year's sampling events) for all parameters.

The first groundwater detection monitoring event was completed during June 1998. When the data are available, the analytical results from this sampling event will be statistically compared to the baseline data. Any statistically significant excursions from baseline values will be reported in subsequent Quarterly Environmental Data Summary reports.

\section{WELDON SPRING QUARRY}

\section{Quarry Water Treatment Plant}

- Samples GW-1035-Q198, GW-1036-Q198, and GW-1040-Q497

Chloride concentrations at the above-listed monitoring locations exceeded baseline values during the first quarter 1998; however, chloride concentrations at these locations have been decreasing since the second quarter 1997. Second quarter 1998 data were not available for this report. 
- Sample GW-1037-Q198

Metal concentrations above baselines were reported in unfiltered samples from this location along the west side of the quarry water treatment plant equalization basin during the first quarter of 1998. Arsenic (12.0 ug/l), chromium ( $41.0 \mathrm{ug} / \mathrm{l})$, and lead $(23.9 \mathrm{ug} / \mathrm{l})$ were above baseline concentrations $(5.5 \mathrm{ug} / 1,7.57 \mathrm{ug} / \mathrm{l}$, and $2.06 \mathrm{ug} / \mathrm{l}$, respectively). Filtered replicates were collected during the sampling event and analytical results show no above-baseline values for any of the metals.

\section{Quarry Vicinity}

Groundwater quality in the quarry vicinity continues to show improvement at locations along the quarry rim for total uranium and nitroaromatic compounds. No above normal values or indications of any increasing contaminant concentrations were reported for the second quarter of 1998.

\section{- St. Charles County Well Field}

No elevated levels of contaminants originating from the Weldon Spring Quarry were reported during the second quarter 1998 data review. Uranium activity results from the last four sampling events at the St. Charles County well field are shown on the attached figure.

\section{SPRINGS}

No elevated contaminants were reported in samples from springs during the second quarter of 1998. Total uranium values at the Burgermeister Spring are plotted versus time on the attached figure.

\section{SURFACE WATER}

No surface water contaminants (at level 2 above normal concentrations) were detected in samples collected during the second quarter of 1998.

\section{AIR}

Data for the monitoring locations are attached in graphic and tabular form. There was one above-normal air monitoring result for the second quarter of 1998.

The gross alpha concentrations for air particulate monitoring for the second quarter 1998 are graphed with the background concentration, (which is based on the average concentration from the 2-year period from the third quarter 1996 thru the second quarter 1998) and background +3 standard deviations shown for comparison. The background location is AP-4012, at the Daniel Boone Elementary School in New Melle.

The gamma exposure from environmental TLD monitoring results for the fourth quarter 1998 are graphed with the background and the background plus 25 mrem quarterly effective dose equivalent (EDE) shown for comparison. The background level is based on monitoring conducted from the beginning of 1998 to the present. The $25 \mathrm{mrem}$ EDE is based on one-fourth of the annual 100 mrem EDE limit for the public established in DOE Order 5400.5. The background locations are TD-4005 (west of the Army site) and TD-4009 (Daniel Boone Elementary School, New Melle). 
The alpha track radon and thoron monitoring results are graphed for the first quarter of 1998 . The background level (based on 1997 monitoring) and the Derived Concentration Guide (DCG) for radon and thoron are shown for comparison. DCGs are reference values established for protection of the public and the environment and listed in DOE Order 5400.5. No monitoring locations had concentrations above the DCG. The background locations are RD-4005 (west of the Army site) and RD-4009 (Daniel Boone Elementary School, New Melle).

One above-normal result for air monitoring that occurred during the second quarter is discussed below.

\section{RAFFINATE PIT 4 AND TSA}

\section{AP-3004-Q298}

During the second quarter 1998, there was one data point considered above normal for site air particulate monitoring. The low volume air particulate (gross alpha) results for location AP3004 were $3.79 \mathrm{E}-15$ and $2.92 \mathrm{E}-15 \mu \mathrm{Ci} / \mathrm{ml}$ for the $22^{\text {nd }}$ and $23^{\text {rd }}$ weeks, respectively. Because these results exceeded the background concentration plus three standard deviations for two consecutive weeks, the above-normal reporting requirements of ES\&H Procedure 1.1.7 were triggered. Station AP-3004 is located south of Pit 4 and west of the Temporary Storage Area (TSA).

Waste handling activities at the TSA, along with a wind direction that was predominately northeasterly during the monitoring period, are the suspected causes for the elevated concentrations. Additional dust control measures at the TSA work area were implemented and the subsequent air monitoring results at AP-3004 have dropped to the normal range below the action level.

\section{SUMMARY}

The previously described data were highlighted as being above prescribed baseline values, varying from historical ranges or being above regulatory limits, and as a result, are subject to more focused attention by the WSSRAP Environmental Protection Group. Continuous trends are monitored to determine the need for additional possible action. Except for the highlighted data, all other indicators subject to reporting in the QEDS were within historic range or below reporting criteria.

If you have any questions, please contact the WSSRAP Community Relations Department at (314) 441-8086.

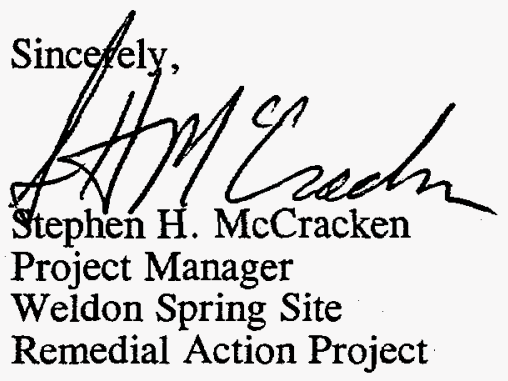





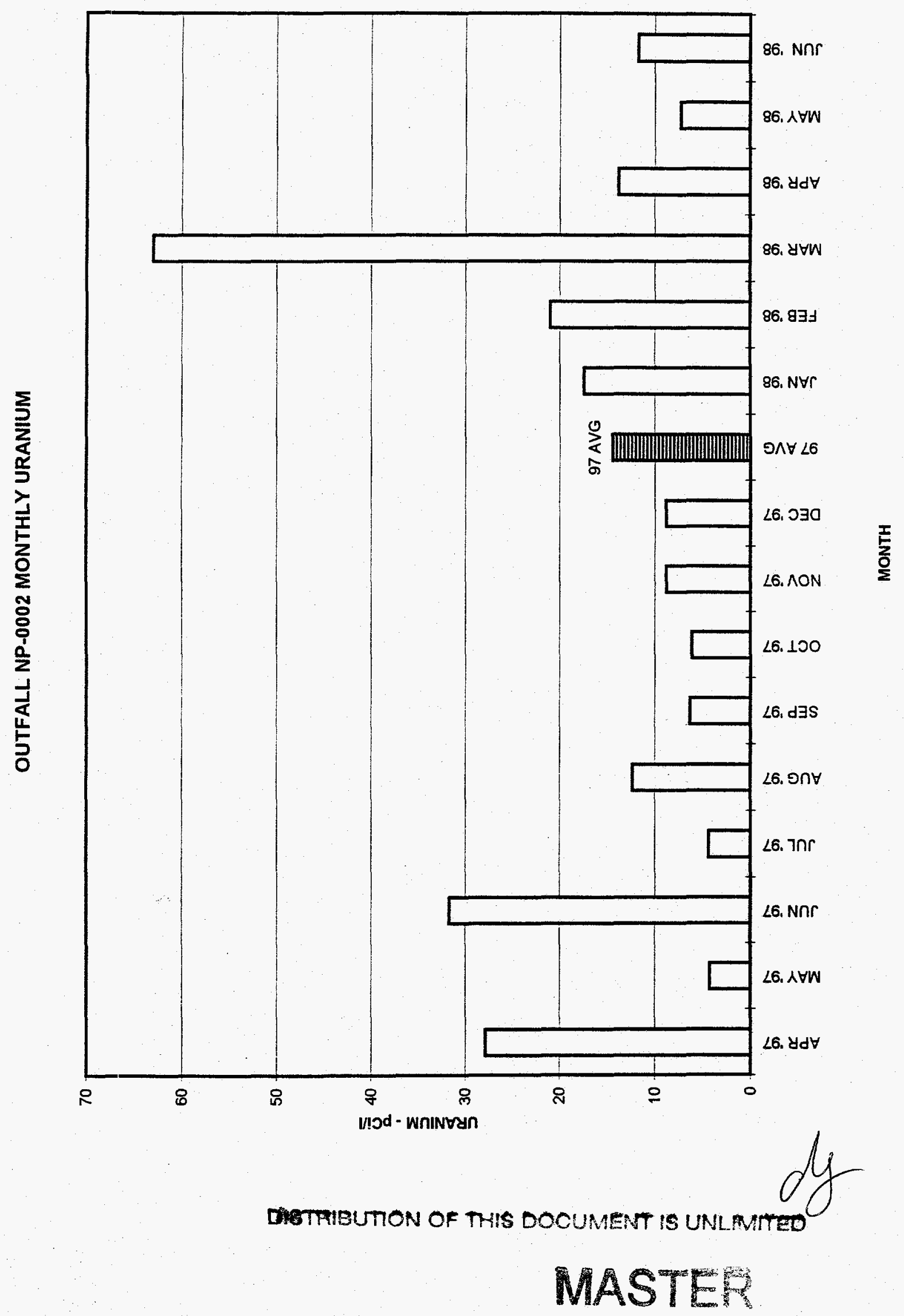




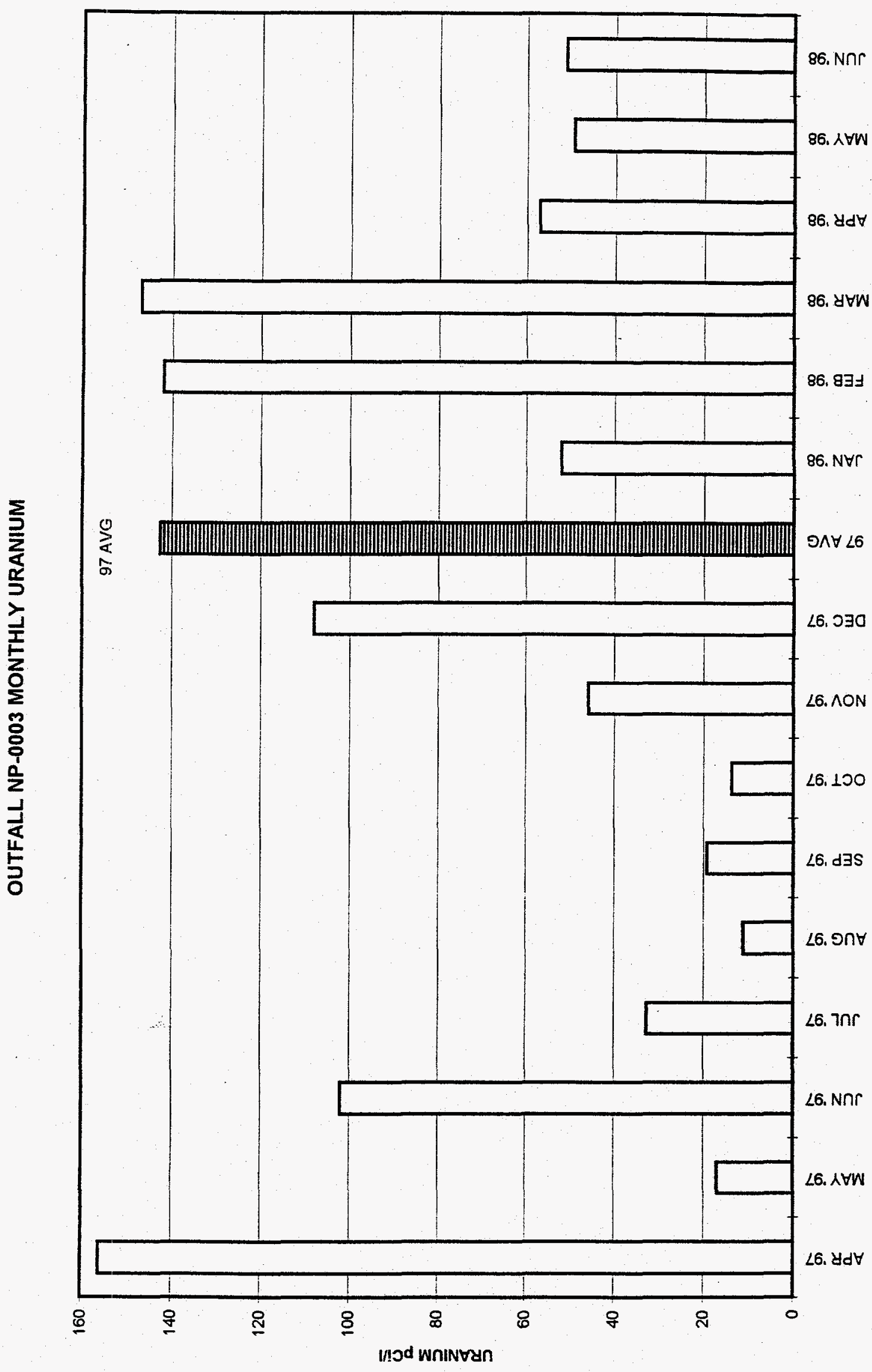

豆 


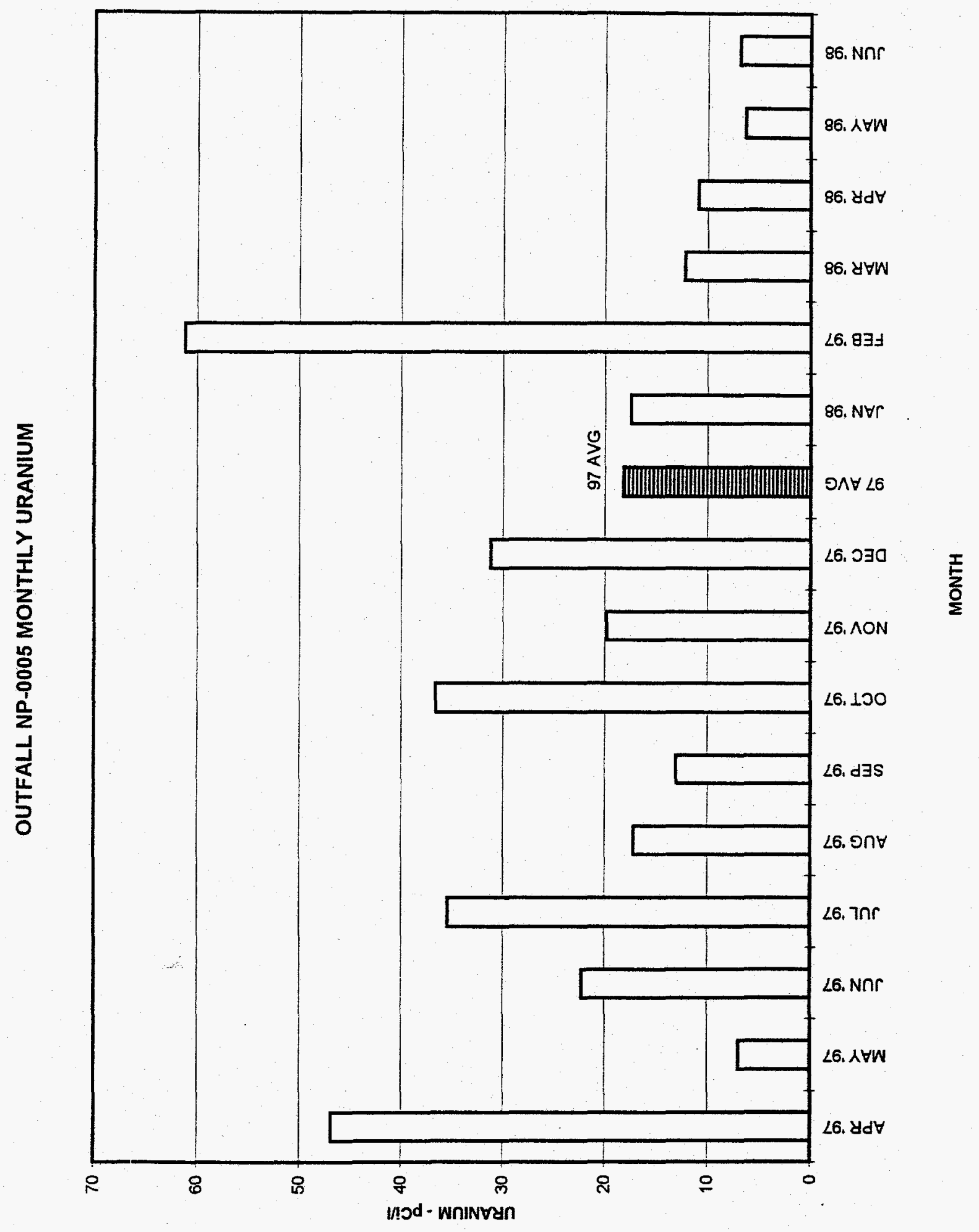


URANIUM ANNUAL AVERAGE

MAJOR NPDES STORM WATER OUTFALLS

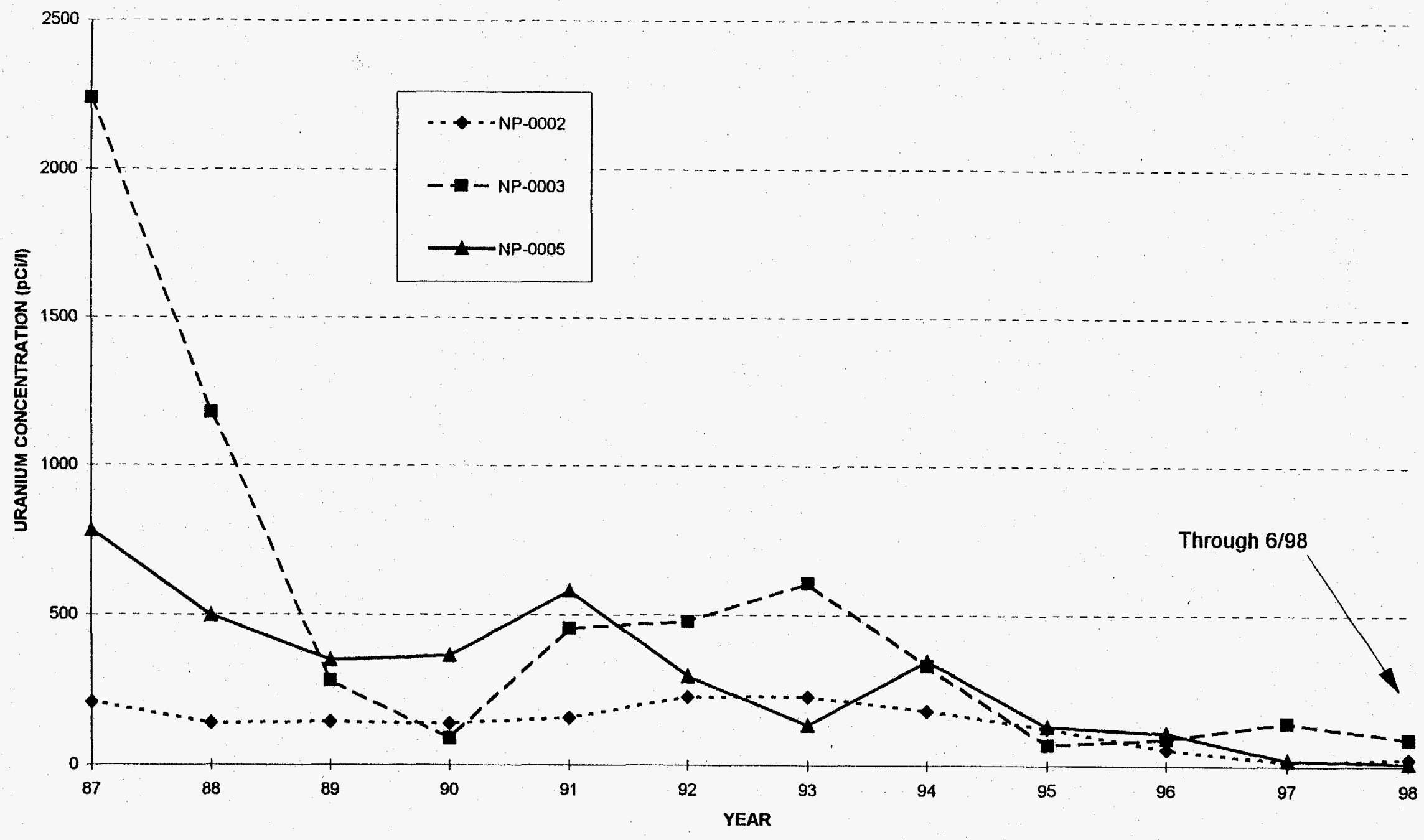


NPDES

DATA MERGED DURING SECOND QUARTER 1998

\begin{tabular}{|c|c|c|c|c|c|c|}
\hline WSSRAP_ID & DATE_.SAM & PARAMETER & CONC & $D L$ & UMITS & METHOD \\
\hline 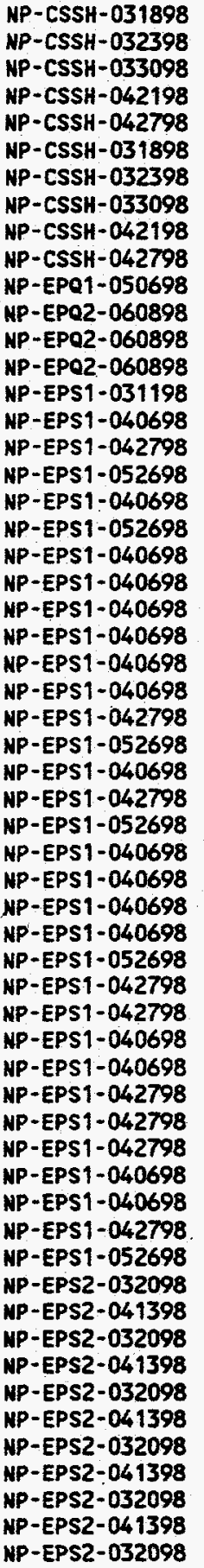 & $\begin{array}{l}03 / 18 / 98 \\
03 / 23 / 98 \\
03 / 30 / 98 \\
04 / 21 / 98 \\
04 / 27 / 98 \\
03 / 18 / 98 \\
03 / 23 / 98 \\
03 / 30 / 98 \\
04 / 21 / 98 \\
04 / 27 / 98 \\
05 / 06 / 98 \\
06 / 08 / 98 \\
06 / 08 / 98 \\
06 / 08 / 98 \\
03 / 11 / 98 \\
04 / 06 / 98 \\
04 / 27 / 98 \\
05 / 26 / 98 \\
04 / 06 / 98 \\
05 / 26 / 98 \\
04 / 06 / 98 \\
04 / 06 / 98 \\
04 / 06 / 98 \\
04 / 06 / 98 \\
04 / 06 / 98 \\
04 / 06 / 98 \\
04 / 27 / 98 \\
05 / 26 / 98 \\
04 / 06 / 98 \\
04 / 27 / 98 \\
05 / 26 / 98 \\
04 / 06 / 98 \\
04 / 06 / 98 \\
04 / 06 / 98 \\
04 / 06 / 98 \\
05 / 26 / 98 \\
04 / 27 / 98 \\
04 / 27 / 98 \\
04 / 06 / 98 \\
04 / 06 / 98 \\
04 / 27 / 98 \\
04 / 27 / 98 \\
04 / 27 / 98 \\
04 / 06 / 98 \\
04 / 06 / 98 \\
04 / 27 / 98 \\
05 / 26 / 98 \\
03 / 20 / 98 \\
04 / 13 / 98 \\
03 / 20 / 98 \\
04 / 13 / 98 \\
03 / 20 / 98 \\
04 / 13 / 98 \\
03 / 20 / 98 \\
04 / 13 / 98 \\
03 / 20 / 98 \\
04 / 13 / 98 \\
03 / 20 / 98\end{array}$ & 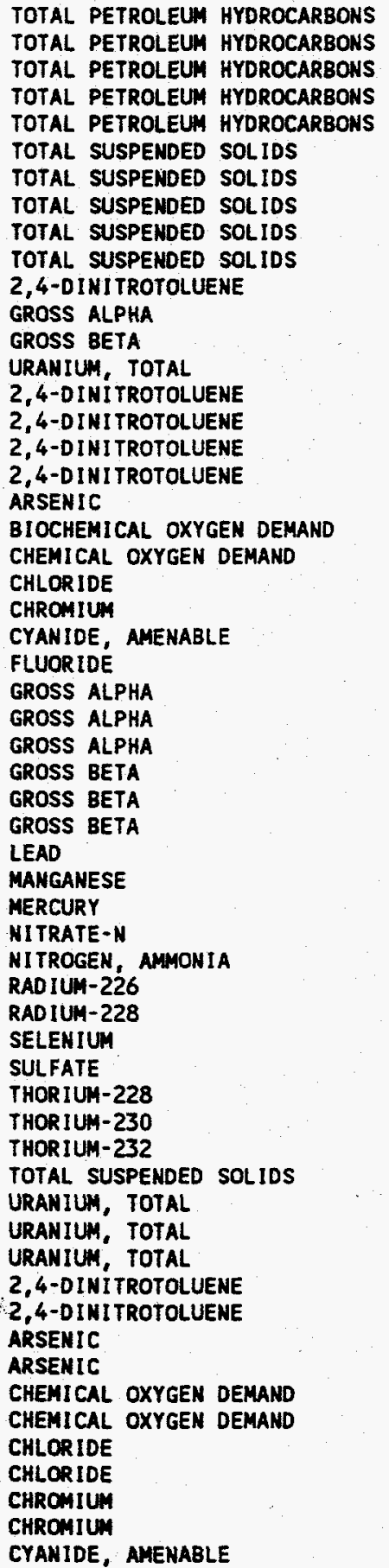 & $\begin{array}{l}\text { ND } \\
\text { ND } \\
\text { ND } \\
\text { ND } \\
\text { ND } \\
\text { ND } \\
\text { ND } \\
34.0 \\
1.0 \\
14 \\
\text { ND } \\
C 0.73 \\
3.33 \\
0.516 \\
\text { ND } \\
\text { ND } \\
\text { ND } \\
\text { ND } \\
\text { ND } \\
4 \\
5.00 \\
57.4 \\
2.0 \\
\text { ND } \\
1.28 \\
5.79 \\
(5.3) \\
(5.93 \\
9.68 \\
13 \\
36.2 \\
\text { ND } \\
1.0 \\
\text { ND } \\
13.3 \\
0.02 \\
0.569 \\
1.7 \\
4.8 \\
162 \\
0.1 \\
\text { ND } \\
0.05 \\
4.00 \\
\text { ND } \\
1.73 \\
2.71 \\
\text { ND } \\
\text { ND } \\
\text { ND } \\
\text { ND } \\
\text { ND } \\
\text { ND } \\
40.6 \\
58.9 \\
2.7 \\
2.3 \\
\text { ND }\end{array}$ & $\begin{array}{l}0.180 \\
0.174 \\
0.186 \\
0.50 \\
7.5 \\
4\end{array}$ & $\begin{array}{l}M G / L \\
M G / L \\
M G / L \\
M G / L \\
M G / L \\
M G / L \\
M G / L \\
M G / L \\
M G / L \\
M G / L \\
U G / L \\
P C I / L \\
P C I / L \\
P C I / L \\
U G / L \\
U G / L \\
U G / L \\
U G / L \\
U G / L \\
M G / L \\
M G / L \\
M G / L \\
U G / L \\
U G / L \\
M G / L \\
P C I / L \\
P C I / L \\
P C I / L \\
P C I / L \\
P C I / L \\
P C I / L \\
U G / L \\
U G / L \\
U G / L \\
M G / L \\
M G / L \\
P C I / L \\
P C I / L \\
U G / L \\
M G / L \\
P C I / L \\
P C I / L \\
P C I / L \\
M G / L \\
P C I / L \\
P C I / L \\
P C I / L \\
U G / L \\
U G / L \\
U G / L \\
U G / L \\
M G / L \\
M G / L \\
M G / L \\
M G / L \\
U G / L \\
U G / L \\
U G / L \\
\end{array}$ & $\begin{array}{l}\text { EPA } 418.1 \\
\text { EPA } 418.1 \\
\text { EPA } 418.1 \\
\text { EPA } 418.1 \\
\text { EPA } 418.1 \\
\text { EPA } 160.2 \\
\text { EPA } 160.2 \\
\text { EPA } 160.2 \\
\text { EPA } 160.2 \\
\text { EPA } 160.2 \\
\text { EPA } 8330 \\
\text { EPA } 900.0 \\
\text { EPA } 900.0 \\
\text { ASTM } 5174-91 \\
\text { SWB46 } 8330 \\
\text { EPA } 8330 \\
\text { EPA } 8330 \\
\text { EPA } 8330 \\
\text { EPA } 200.7 \\
\text { EPA } 405.1 \\
\text { EPA } 410.4 \\
\text { EPA } 300.0 \\
\text { EPA } 200.7 \\
\text { EPA } 9010 \\
\text { EPA } 300.0 \\
\text { EPA } 900.0 \\
\text { EPA } 900.0 \\
\text { EPA } 900.0 \\
\text { EPA } 900.0 \\
\text { EPA } 900.0 \\
\text { EPA } 900.0 \\
\text { EPA } 200.7 \\
\text { EPA } 200.7 \\
\text { EPA } 245.1 \\
\text { EPA } 353.1 \\
\text { EPA } 350.1 \\
\text { SM-705 } \\
\text { PERC/BRO0KS } \\
\text { EPA } 200.7 \\
\text { EPA } 300.0 \\
\text { USAEC } \\
\text { USAEC } \\
\text { USAEC } \\
\text { EPA } 160.2 \\
\text { ASTM } 5174-91 \\
\text { ASTM } 02907 \\
\text { ASTM } 5174-91 \\
\text { USATHAMA } \\
\text { USATHAMA } \\
\text { EPA } 200.7 \\
\text { EPA } 200.7 \\
\text { EPA } 410.4 \\
\text { EPA } 410.4 \\
\text { EPA } 300.0 \\
\text { EPA } 300.0 \\
\text { EPA } 200.7 \\
\text { EPA } 200.7 \\
\text { EPA } 9010 \\
\end{array}$ \\
\hline
\end{tabular}




\begin{tabular}{|c|c|c|c|c|c|c|}
\hline WSSRAP_ID & DATE_SAM & PARAMETER & CONC & $\mathrm{DL}$ & UNITS & METHOD \\
\hline 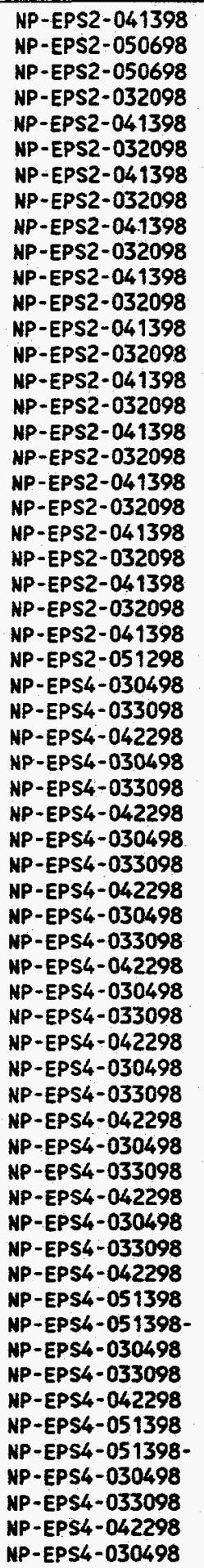 & $\begin{array}{l}04 / 13 / 98 \\
05 / 06 / 98 \\
05 / 06 / 98 \\
03 / 20 / 98 \\
04 / 13 / 98 \\
03 / 20 / 98 \\
04 / 13 / 98 \\
03 / 20 / 98 \\
04 / 13 / 98 \\
03 / 20 / 98 \\
04 / 13 / 98 \\
03 / 20 / 98 \\
04 / 13 / 98 \\
03 / 20 / 98 \\
04 / 13 / 98 \\
03 / 20 / 98 \\
04 / 13 / 98 \\
03 / 20 / 98 \\
04 / 13 / 98 \\
03 / 20 / 98 \\
04 / 13 / 98 \\
03 / 20 / 98 \\
04 / 13 / 98 \\
03 / 20 / 98 \\
04 / 13 / 98 \\
05 / 12 / 98 \\
03 / 04 / 98 \\
03 / 30 / 98 \\
04 / 22 / 98 \\
03 / 04 / 98 \\
03 / 30 / 98 \\
04 / 22 / 98 \\
03 / 04 / 98 \\
03 / 30 / 98 \\
04 / 22 / 98 \\
03 / 04 / 98 \\
03 / 30 / 98 \\
04 / 22 / 98 \\
03 / 04 / 98 \\
03 / 30 / 98 \\
04 / 22 / 98 \\
03 / 04 / 98 \\
03 / 30 / 98 \\
04 / 22 / 98 \\
03 / 04 / 98 \\
03 / 30 / 98 \\
04 / 22 / 98 \\
03 / 04 / 98 \\
03 / 30 / 98 \\
04 / 22 / 98 \\
05 / 13 / 98 \\
05 / 13 / 98 \\
03 / 04 / 98 \\
03 / 30 / 98 \\
04 / 22 / 98 \\
05 / 13 / 98 \\
05 / 13 / 98 \\
03 / 04 / 98 \\
03 / 30 / 98 \\
04 / 22 / 98 \\
03 / 04 / 98\end{array}$ & 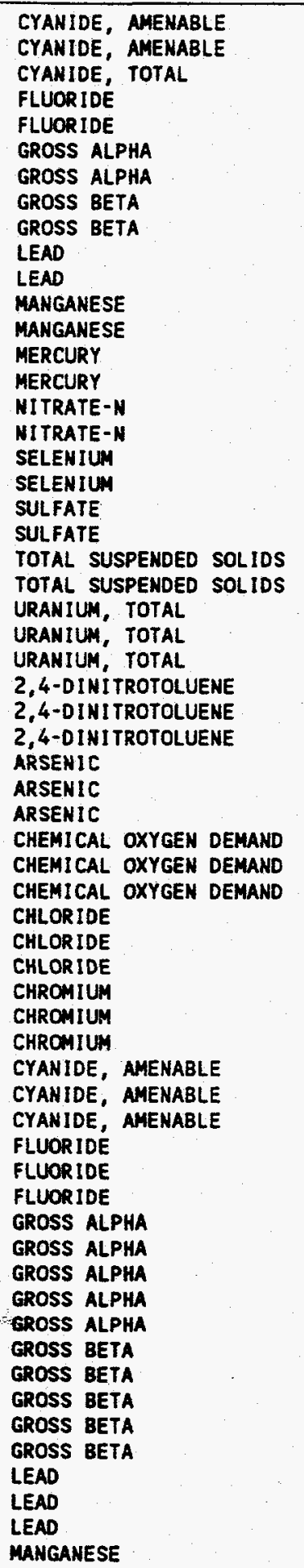 & $\begin{array}{l}\text { ND } \\
\text { ND } \\
\text { ND } \\
1.56 \\
1.20 \\
1.81 \\
6.56 \\
7.52 \\
13.2 \\
\text { ND } \\
\text { ND } \\
1.2 \\
1.4 \\
\text { ND } \\
\text { ND } \\
27.7 \\
13.6 \\
6.7 \\
6.5 \\
226 \\
242 \\
6.00 \\
2.00 \\
\text { ND } \\
\text { ND } \\
13.0 \\
0.04 \\
\text { ND } \\
\text { ND } \\
4.0 \\
\text { ND } \\
2.6 \\
\text { ND } \\
\text { ND } \\
5.0 \\
75 \\
44.1 \\
52.2 \\
2.6 \\
1.5 \\
2.4 \\
\text { ND } \\
\text { ND } \\
\text { ND } \\
1.7 \\
1.56 \\
1.71 \\
4.63 \\
4.51 \\
12.0 \\
57.2 \\
13.0 \\
13.0 \\
9.20 \\
17.0 \\
134 \\
36.1 \\
2.5 \\
1.0 \\
\text { ND }\end{array}$ & $\begin{array}{l}5.00 \\
5.00 \\
5.00 \\
0.10 \\
0.10 \\
0.891 \\
0.739 \\
0.829 \\
0.805 \\
0.90 \\
1.1 \\
0.30 \\
0.70 \\
0.10 \\
0.10 \\
2.50 \\
1.00 \\
2.2 \\
3.1 \\
10.0 \\
10.0 \\
1.00 \\
1.00 \\
0.67 \\
0.67 \\
0.02 \\
0.00 \\
0\end{array}$ & $\begin{array}{l}U G / L \\
U G / L \\
U G / L \\
M G / L \\
M G / L \\
P C I / L \\
P C I / L \\
P C I / L \\
P C I / L \\
U G / L \\
U G / L \\
U G / L \\
U G / L \\
U G / L \\
U G / L \\
M G / L \\
M G / L \\
U G / L \\
U G / L \\
M G / L \\
M G / L \\
M G / L \\
M G / L \\
P C I / L \\
P C I / L \\
P C I / L \\
U G / L \\
U G / L \\
U G / L \\
U G / L \\
U G / L \\
U G / L \\
M G / L \\
M G / L \\
M G / L \\
M G / L \\
M G / L \\
M G / L \\
U G / L \\
U G / L \\
U G / L \\
U G / L \\
U G / L \\
U G / L \\
M G / L \\
M G / L \\
M G / L \\
P C I / L \\
P C I / L \\
P C I / L \\
P C I / L \\
P C I / L \\
P C I / L \\
P C I / L \\
P C I / L \\
P C I / L \\
P C I / L \\
U G / L \\
U G / L \\
U G / L \\
U G / L\end{array}$ & $\begin{array}{l}\text { EPA } 9010 \\
\text { EPA } 9010 \\
\text { EPA } 335.2 \\
\text { EPA } 300.0 \\
\text { EPA } 300.0 \\
\text { EPA } 900.0 \\
\text { EPA } 900.0 \\
\text { EPA } 900.0 \\
\text { EPA } 900.0 \\
\text { EPA } 200.7 \\
\text { EPA } 200.7 \\
\text { EPA } 200.7 \\
\text { EPA } 200.7 \\
\text { EPA } 245.1 \\
\text { EPA } 245.1 \\
\text { EPA } 353.1 \\
\text { EPA } 353.1 \\
\text { EPA } 200.7 \\
\text { EPA } 200.7 \\
\text { EPA } 300.0 \\
\text { EPA } 300.0 \\
\text { EPA } 160.2 \\
\text { EPA } 160.2 \\
\text { ASTM } 5174-91 \\
\text { ASTM } 5174-91 \\
\text { ASTM } 5174-91 \\
\text { EPA } 8330 \\
\text { USATHAMA } \\
\text { EPA } 8330 \\
\text { EPA } 200.7 \\
\text { EPA } 6010 A \\
\text { EPA } 6010 A \\
\text { EPA } 410.0 \\
\text { EPA } 410.4 \\
\text { EPA } 410.4 \\
\text { EPA } 300.0 \\
\text { EPA } 300.0 \\
\text { EPA } 300.0 \\
\text { EPA } 200.7 \\
\text { EPA } 6010 A \\
\text { EPA } 6010 A \\
\text { EPA } 335.1 \\
\text { EPA } 9010 \\
\text { EPA } 9010 \\
\text { EPA } 300.0 \\
\text { EPA } 300.0 \\
\text { EPA } 300.0 \\
\text { EPA } 900.0 \\
\text { EPA } 900.0 \\
\text { EPA } 900.0 \\
\text { EPA } 900.0 \\
\text { EPA } 900.0 \\
\text { EPA } 900.0 \\
\text { EPA } 900.0 \\
\text { EPA } 900.0 \\
\text { EPA } 900.0 \\
\text { EPA } 900.0 \\
\text { EPA } 200.7 \\
\text { EPA } 6010 A \\
\text { EPA } 6010 A \\
\text { EPA } 200.7 \\
\end{array}$ \\
\hline
\end{tabular}




\begin{tabular}{|c|c|c|c|c|c|c|}
\hline HSSRAP_ID & DATE_SAM & PARAMETER & CONC & $D L$ & UNITS & METHOD \\
\hline $\begin{array}{l}\text { NP-EPS4-033098 } \\
\text { NP-EPS4-042298 } \\
\text { NP-EPS4-030498 } \\
\text { NP-EPS4-033098 } \\
\text { NP-EPS4-042298 } \\
\text { NP-EPS4-030498 } \\
\text { NP-EPS4-033098 } \\
\text { NP-EPS4-042298 } \\
\text { NP-EPS4-030498 } \\
\text { NP-EPS4-033098 } \\
\text { NP-EPS4-030498 } \\
\text { NP-EPS4-033098 } \\
\text { NP-EPS4-030498 } \\
\text { NP-EPS4-033098 } \\
\text { NP-EPS4-042298 } \\
\text { NP-EPS4-030498 } \\
\text { NP-EPS4-033098 } \\
\text { NP-EPS4-042298 } \\
\text { NP-EPS4-030498 } \\
\text { NP-EPS4-033098 } \\
\text { NP-EPS4-030498 } \\
\text { NP-EPS4-033098 } \\
\text { NP-EPS4-030498 } \\
\text { NP-EPS4-033098 } \\
\text { NP-EPS4-030498 } \\
\text { NP-EPS4-033098 } \\
\text { NP-EPS4-042298 } \\
\text { NP-EPS4-030498 } \\
\text { NP-EPS4-033098 } \\
\text { NP-EPS4-042298 } \\
\text { NP-EPS4-051398 } \\
\text { NP-EPS4-051398- }\end{array}$ & $\begin{array}{l}03 / 30 / 98 \\
04 / 22 / 98 \\
03 / 04 / 98 \\
03 / 30 / 98 \\
04 / 22 / 98 \\
03 / 04 / 98 \\
03 / 30 / 98 \\
04 / 22 / 98 \\
03 / 04 / 98 \\
03 / 30 / 98 \\
03 / 04 / 98 \\
03 / 30 / 98 \\
03 / 04 / 98 \\
03 / 30 / 98 \\
04 / 22 / 98 \\
03 / 04 / 98 \\
03 / 30 / 98 \\
04 / 22 / 98 \\
03 / 04 / 98 \\
03 / 30 / 98 \\
03 / 04 / 98 \\
03 / 30 / 98 \\
03 / 04 / 98 \\
03 / 30 / 98 \\
03 / 04 / 98 \\
03 / 30 / 98 \\
04 / 22 / 98 \\
03 / 04 / 98 \\
03 / 30 / 98 \\
04 / 22 / 98 \\
05 / 13 / 98 \\
05 / 13 / 98\end{array}$ & $\begin{array}{l}\text { MANGANESE } \\
\text { MANGANESE } \\
\text { MERCURY } \\
\text { MERCURY } \\
\text { MERCURY } \\
\text { NITRATE-N } \\
\text { NITRATE-N } \\
\text { NITRATE-N } \\
\text { RADIUM-226 } \\
\text { RADIUM-226 } \\
\text { RADIUM-228 } \\
\text { RADIUM-228 } \\
\text { SELENIUM } \\
\text { SELENIUM } \\
\text { SELENIUM } \\
\text { SULFATE } \\
\text { SULFATE } \\
\text { SULFATE } \\
\text { THORIUM-228 } \\
\text { THORIUM-228 } \\
\text { THORIUM-230 } \\
\text { THORIUA-230 } \\
\text { THORIUM-232 } \\
\text { THORIUM-232 } \\
\text { TOTAL SUSPENDED SOLIDS } \\
\text { TOTAL SUSPENDED SOLIDS } \\
\text { TOTAL SUSPENDED SOLIDS } \\
\text { URANIUM, TOTAL } \\
\text { URANIUM, TOTAL } \\
\text { URANIUM, TOTAL } \\
\text { URANIUM, TOTAL } \\
\text { URANIUM, TOTAL }\end{array}$ & $\begin{array}{l}1.1 \\
1.8 \\
\text { ND } \\
\text { ND } \\
\text { ND } \\
46 \\
18 \\
19.6 \\
1.56 \\
1.25 \\
2.22 \\
3.19 \\
16 \\
3.2 \\
6.1 \\
430 \\
134 \\
162 \\
10.07 \\
10.05 \\
0.203 \\
60.08 \\
\text { ND } \\
60.00 \\
\text { ND } \\
2.00 \\
2.0 \\
\text { ND } \\
\text { ND } \\
0.71 \\
8.40 \\
8.35\end{array}$ & $\begin{array}{l}0.04 \\
0.7 \\
0.1 \\
0.1 \\
0.10 \\
0.1 \\
1.25 \\
1.25 \\
0.137 \\
0.103 \\
0.370 \\
0.504 \\
3.4 \\
0.52 \\
3.1 \\
2.0 \\
5.00 \\
10.0 \\
0.082 \\
0.126 \\
0.040 \\
0.118 \\
0.040 \\
0.092 \\
4.0 \\
1.00 \\
1.00 \\
0.677 \\
0.677 \\
0.677 \\
0.0203 \\
0.0203\end{array}$ & $\begin{array}{l}U G / L \\
U G / L \\
U G / L \\
U G / L \\
U G / L \\
M G / L \\
M G / L \\
M G / L \\
P C I / L \\
P C I / L \\
P C I / L \\
P C I / L \\
U G / L \\
U G / L \\
U G / L \\
M G / L \\
M G / L \\
M G / L \\
P C I / L \\
P C I / L \\
P C I / L \\
P C I / L \\
P C I / L \\
P C I / L \\
M G / L \\
M G / L \\
M G / L \\
P C I / L \\
P C I / L \\
P C I / L \\
P C I / L \\
P C I / L\end{array}$ & $\begin{array}{l}\text { EPA } 6010 A \\
\text { EPA } 6010 A \\
\text { EPA } 245.1 \\
\text { EPA } 7470 A \\
\text { EPA } 245.1 \\
\text { EPA } 300.0 \\
\text { EPA } 353.1 \\
\text { EPA } 353.1 \\
\text { EPA } 904.0 \\
\text { EPA } 904.0 \\
\text { EPA } 904.0 \\
\text { EPA } 904.0 \\
\text { EPA } 200.7 \\
\text { EPA } 6010 A \\
\text { EPA } 6010 A \\
\text { EPA } 300.0 \\
\text { EPA } 300.0 \\
\text { EPA } 300.0 \\
\text { NAS-NS-3004 } \\
\text { NAS-NS-3004 } \\
\text { NAS-NS-3004 } \\
\text { NAS-NS-3004 } \\
\text { NAS-NS-3004 } \\
\text { NAS-NS-3004 } \\
\text { EPA } 160.2 \\
\text { EPA } 160.2 \\
\text { EPA } 160.2 \\
\text { ASTM } 5174-91 \\
\text { ASTM } 5174-91 \\
\text { ASTM } 5174-91 \\
\text { ASTM } 5174-91 \\
\text { ASTM } 5174\end{array}$ \\
\hline
\end{tabular}




\section{GROUNDWATER}


TOTAL URANIUM ACTIVITY, UPGRADIENT OF PUMPING WELLS AND AT THE ST.

CHARLES COUNTY WATER TREATMENT FACILITY

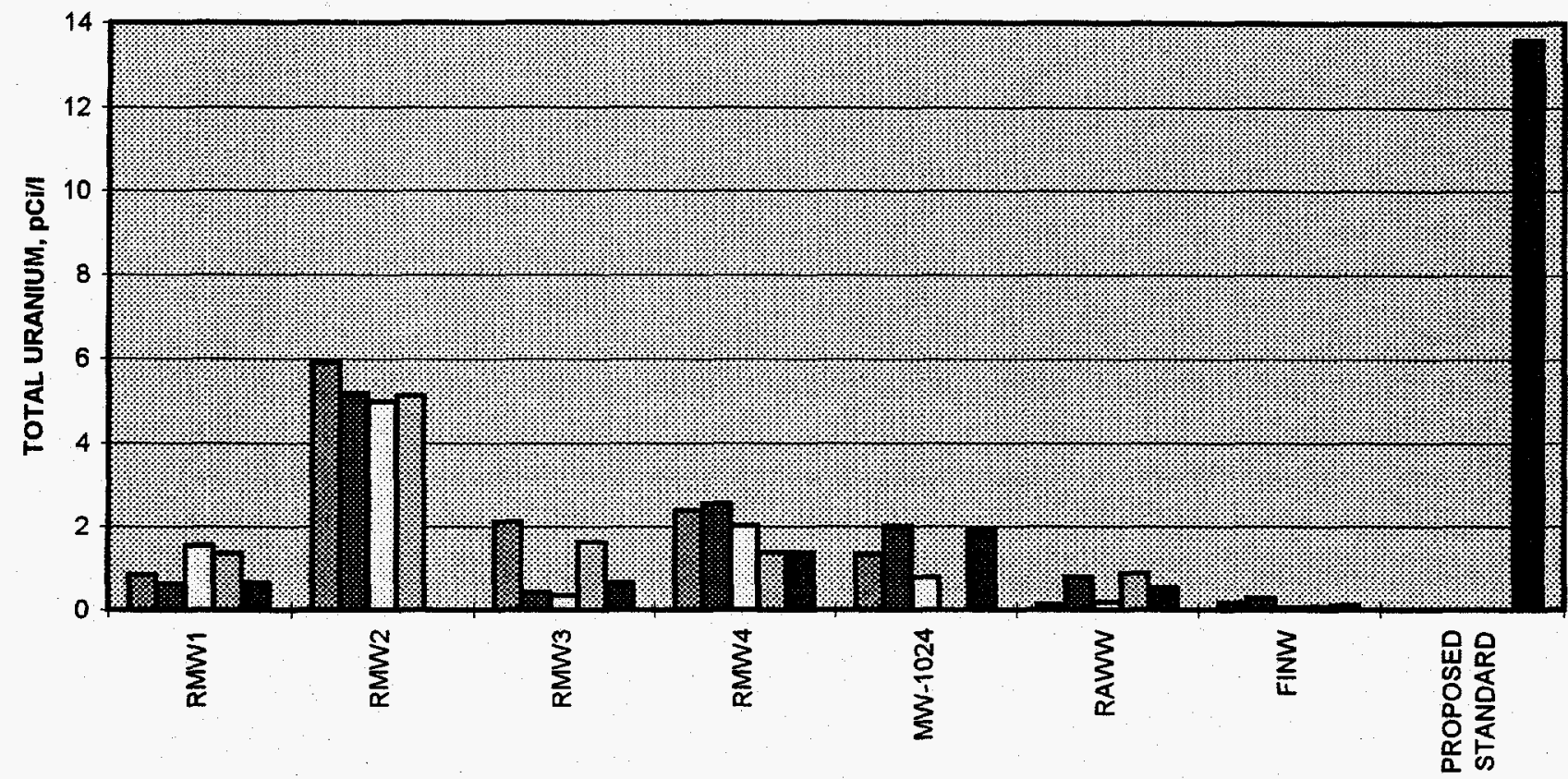

MONITORING LOCATION

TOTAL URANIUM ACTIVITY, ST. CHARLES COUNTY WATER PRODUCTION WELLS

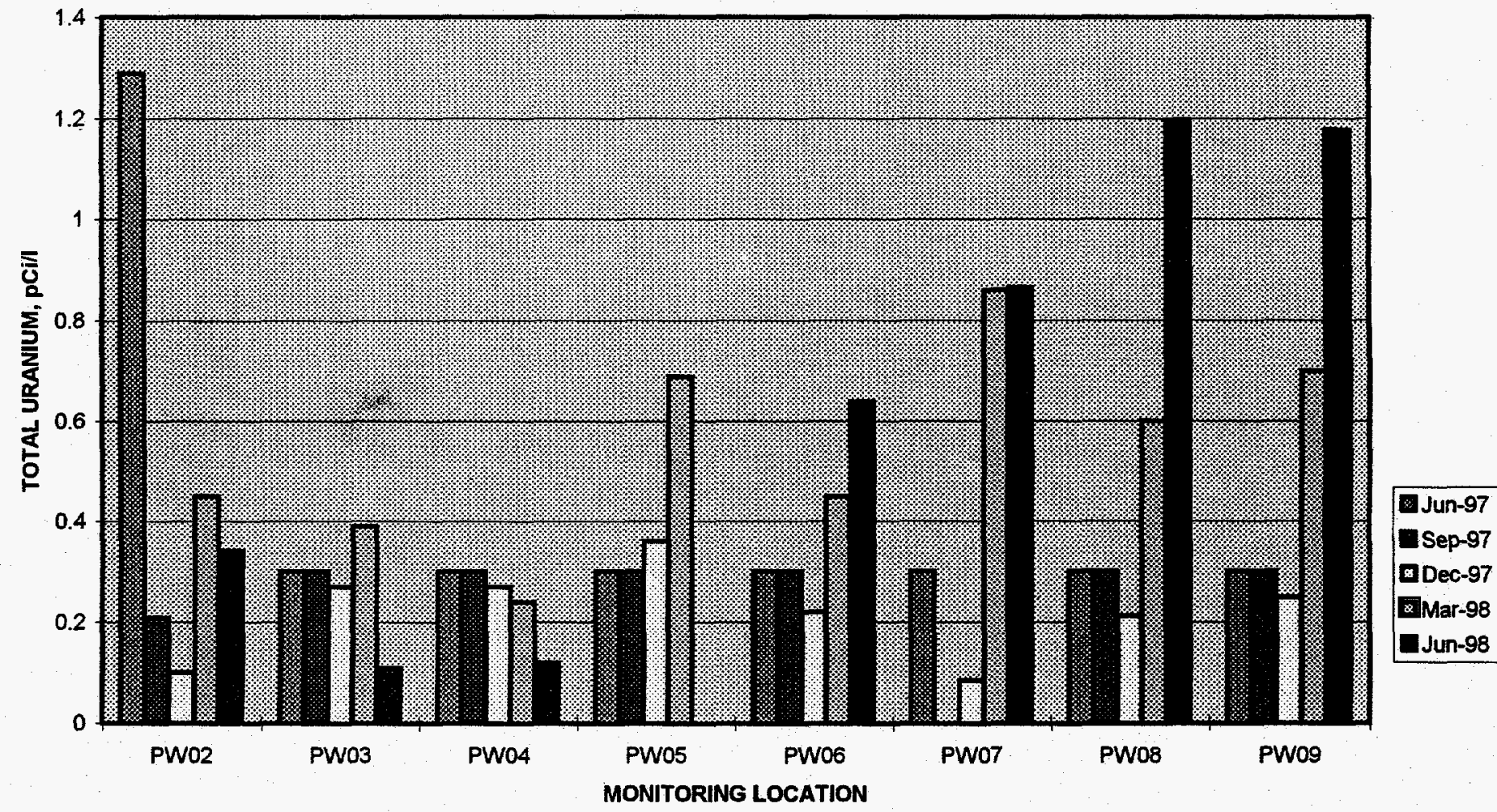


GROUNDWATER

DATA MERGED DURING SECOND QUARTER 1998

\begin{tabular}{|c|c|c|c|c|c|c|}
\hline WSSRAP_ID & DATE_SAM & PARAMETER & CONC & DL & UNITS & METHOD \\
\hline $\begin{array}{l}G W-1002-0298 \\
G W-1002-0298 \\
G W-1002-0298 \\
G W-1002-0298 \\
G W-1002-0298 \\
G W-1002-0298 \\
G W-1004-0298 \\
G W-1004-0298 \\
G W-1004-0298 \\
G W-1004-0298 \\
G W-1004-0298 \\
G W-1004-0298 \\
G W-1006-0298 \\
G W-1006-0298 \\
G W-1006-0298 \\
G W-1006-0298 \\
G W-1006-0298 \\
G W-1006-0298 \\
G W-1007-0298 \\
G W-1007-0298 \\
G W-1007-0298 \\
G W-1007-0298 \\
G W-1007-0298 \\
G W-1007-0298 \\
G W-1008-0298 \\
G W-1008-0298 \\
G W-1008-0298 \\
G W-1008-0298 \\
G W-1008-0298 \\
G W-1008-0298 \\
G W-1009-0298 \\
G W-1009-0298 \\
G W-1009-0298 \\
G W-1009-0298 \\
G W-1009-0298 \\
G W-1009-0298 \\
G W-1013-0298 \\
G W-1013-0298 \\
G W-1013-0298 \\
G W-1013-0298 \\
G W-1013-02298 \\
G W-1013-02298 \\
G W-1014-00298 \\
G W-1014-0298 \\
G W-1014-0298 \\
G W-1014-02988 \\
G H-1014-00298 \\
G W-1014-0298 \\
G W-1024-0198 \\
G W-1027-0198 \\
G W-1028-0298 \\
G W-1028-02298 \\
G W-1028-00298 \\
G W-1028-0298 \\
G W-1028-0298 \\
G W-1028-0298 \\
G W-1029-0298 \\
G W-1029-02298\end{array}$ & $\begin{array}{l}04 / 27 / 98 \\
04 / 27 / 98 \\
04 / 27 / 98 \\
04 / 27 / 98 \\
04 / 27 / 98 \\
04 / 27 / 98 \\
04 / 27 / 98 \\
04 / 27 / 98 \\
04 / 27 / 98 \\
04 / 27 / 98 \\
04 / 27 / 98 \\
04 / 27 / 98 \\
04 / 28 / 98 \\
04 / 28 / 98 \\
04 / 28 / 98 \\
04 / 28 / 98 \\
04 / 28 / 98 \\
04 / 28 / 98 \\
04 / 28 / 98 \\
04 / 28 / 98 \\
04 / 28 / 98 \\
04 / 28 / 98 \\
04 / 28 / 98 \\
04 / 28 / 98 \\
04 / 23 / 98 \\
04 / 23 / 98 \\
04 / 23 / 98 \\
04 / 23 / 98 \\
04 / 23 / 98 \\
04 / 23 / 98 \\
04 / 23 / 98 \\
04 / 23 / 98 \\
04 / 23 / 98 \\
04 / 23 / 98 \\
04 / 23 / 98 \\
04 / 23 / 98 \\
04 / 23 / 98 \\
04 / 23 / 98 \\
04 / 23 / 98 \\
04 / 23 / 98 \\
04 / 23 / 98 \\
04 / 23 / 98 \\
04 / 23 / 98 \\
04 / 23 / 98 \\
04 / 23 / 98 \\
04 / 23 / 98 \\
04 / 23 / 98 \\
04 / 23 / 98 \\
03 / 24 / 98 \\
01 / 27 / 98 \\
04 / 23 / 98 \\
04 / 23 / 98 \\
04 / 23 / 98 \\
04 / 23 / 98 \\
04 / 23 / 98 \\
04 / 23 / 98 \\
04 / 27 / 98 \\
04 / 27 / 98\end{array}$ & 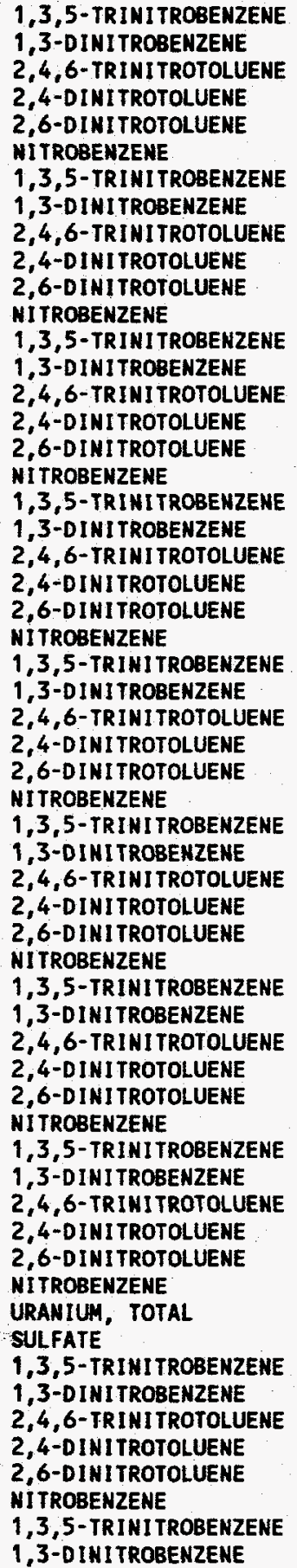 & 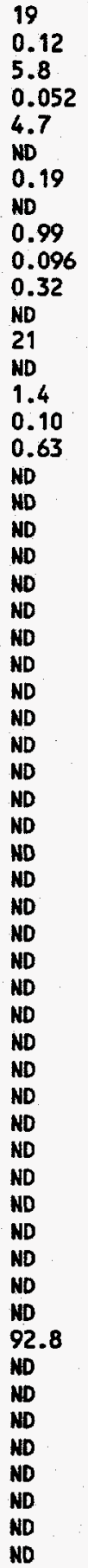 & $\begin{array}{l}0.030 \\
0.090 \\
0.030 \\
0.030 \\
0.010 \\
0.030 \\
0.030 \\
0.090 \\
0.030 \\
0.030 \\
0.010 \\
0.030 \\
0.030 \\
0.090 \\
0.030 \\
0.030 \\
0.010 \\
0.030 \\
0.030 \\
0.090 \\
0.030 \\
0.030 \\
0.010 \\
0.030 \\
0.030 \\
0.090 \\
0.030 \\
0.030 \\
0.010 \\
0.030 \\
0.030 \\
0.090 \\
0.030 \\
0.030 \\
0.010 \\
0.030 \\
0.030 \\
0.090 \\
0.030 \\
0.030 \\
0.010 \\
0.030 \\
0.030 \\
0.090 \\
0.030 \\
0.030 \\
0.010 \\
0.030 \\
0.677 \\
5.00 \\
0.030 \\
0.090 \\
0.030 \\
0.030 \\
0.010 \\
0.030 \\
0.030 \\
0.090\end{array}$ & 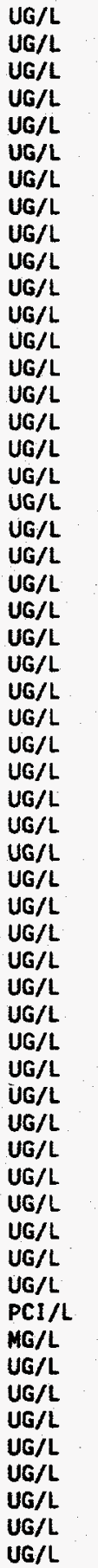 & $\begin{array}{l}\text { USATHAMA } \\
\text { USATHAMA } \\
\text { USATHAMA } \\
\text { USATHAMA } \\
\text { USATHAMA } \\
\text { USATHAMA } \\
\text { USATHAMA } \\
\text { USATHAMA } \\
\text { USATHAMA } \\
\text { USATHAMA } \\
\text { USATHAMA } \\
\text { USATHAMA } \\
\text { USATHAMA } \\
\text { USATHAMA } \\
\text { USATHAMA } \\
\text { USATHAMA } \\
\text { USATHAMA } \\
\text { USATHAMA } \\
\text { USATHAMA } \\
\text { USATHAMA } \\
\text { USATHAMA } \\
\text { USATHAMA } \\
\text { USATHAMA } \\
\text { USATHAMA } \\
\text { USATHAMA } \\
\text { USATHAMA } \\
\text { USATHAMA } \\
\text { USATHAMA } \\
\text { USATHAMA } \\
\text { USATHAMA } \\
\text { USATHAMA } \\
\text { USATHAMA } \\
\text { USATHAMA } \\
\text { USATHAMA } \\
\text { USATHAMA } \\
\text { USATHAMA } \\
\text { USATHAMA } \\
\text { USATHAMA } \\
\text { USATHAMA } \\
\text { USATHAMA } \\
\text { USATHAMA } \\
\text { USATHAMA } \\
\text { USATHAMA } \\
\text { USATHAMA } \\
\text { USATHAMA } \\
\text { USATHAMA } \\
\text { USATHAMA } \\
\text { USATHAMA } \\
\text { ASTH } 5174-91 \\
\text { EPA } 300.0 \\
\text { USATHAMA } \\
\text { USATHAMA } \\
\text { USATHAMA } \\
\text { USATHAMA } \\
\text { USATHAMA } \\
\text { USATHAMA } \\
\text { USATHAMA } \\
\text { USATHAMA } \\
\end{array}$ \\
\hline
\end{tabular}




\begin{tabular}{|c|c|c|c|c|c|c|c|}
\hline USSRAP_ID & DATE_SAM & PARAMETER & CONC & $\mathrm{DL}$ & UNITS & METHOD & \\
\hline 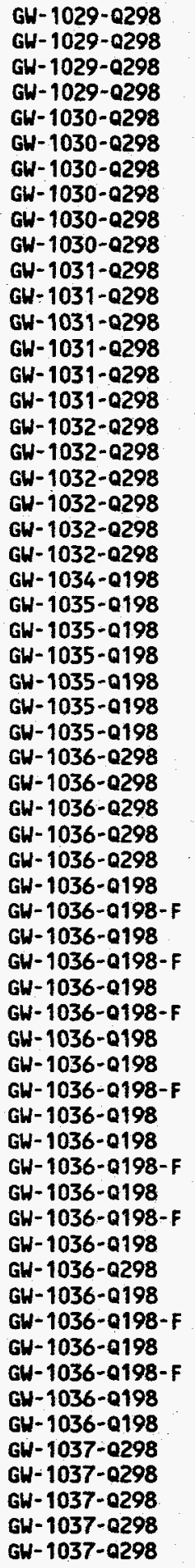 & $\begin{array}{l}04 / 27 / 98 \\
04 / 27 / 98 \\
04 / 27 / 98 \\
04 / 27 / 98 \\
04 / 27 / 98 \\
04 / 27 / 98 \\
04 / 27 / 98 \\
04 / 27 / 98 \\
04 / 27 / 98 \\
04 / 27 / 98 \\
04 / 23 / 98 \\
04 / 23 / 98 \\
04 / 23 / 98 \\
04 / 23 / 98 \\
04 / 23 / 98 \\
04 / 23 / 98 \\
04 / 28 / 98 \\
04 / 28 / 98 \\
04 / 28 / 98 \\
04 / 28 / 98 \\
04 / 28 / 98 \\
04 / 28 / 98 \\
01 / 28 / 98 \\
01 / 23 / 98 \\
01 / 23 / 98 \\
01 / 23 / 98 \\
01 / 23 / 98 \\
01 / 23 / 98 \\
01 / 23 / 98 \\
04 / 21 / 98 \\
04 / 21 / 98 \\
04 / 21 / 98 \\
04 / 21 / 98 \\
04 / 21 / 98 \\
01 / 27 / 98 \\
01 / 27 / 98 \\
01 / 27 / 98 \\
01 / 27 / 98 \\
01 / 27 / 98 \\
01 / 27 / 98 \\
01 / 27 / 98 \\
01 / 27 / 98 \\
01 / 27 / 98 \\
01 / 27 / 98 \\
01 / 27 / 98 \\
01 / 27 / 98 \\
01 / 27 / 98 \\
01 / 27 / 98 \\
01 / 27 / 98 \\
04 / 21 / 98 \\
01 / 27 / 98 \\
01 / 27 / 98 \\
01 / 27 / 98 \\
01 / 27 / 98 \\
01 / 27 / 98 \\
01 / 27 / 98 \\
04 / 21 / 98 \\
04 / 21 / 98 \\
04 / 21 / 98 \\
04 / 21 / 98 \\
04 / 21 / 98\end{array}$ & 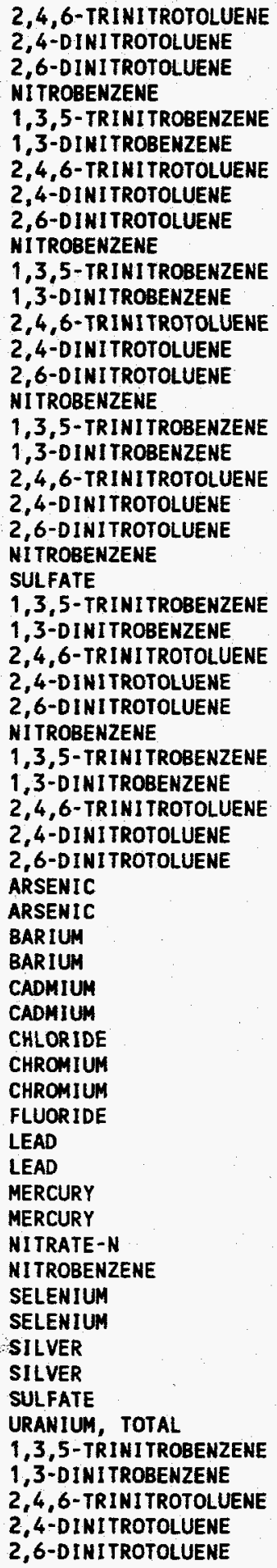 & $\begin{array}{l}\text { ND } \\
\text { ND } \\
\text { ND } \\
\text { ND } \\
\text { ND } \\
\text { ND } \\
\text { ND } \\
\text { ND } \\
\text { ND } \\
\text { ND } \\
\text { ND } \\
\text { ND } \\
\text { ND } \\
\text { ND } \\
\text { ND } \\
\text { ND } \\
\text { ND } \\
\text { S2.6 } \\
\text { ND } \\
\text { ND } \\
\text { ND } \\
\text { ND } \\
\text { ND } \\
\text { ND } \\
\text { ND } \\
\text { ND } \\
\text { ND } \\
\text { ND } \\
\text { ND } \\
3.0 \\
2.0 \\
197 \\
162 \\
\text { ND } \\
\text { ND } \\
\text { ND } \\
\text { ND } \\
\text { ND } \\
3.0 \\
0.31 \\
\text { ND } \\
\text { ND } \\
\text { ND } \\
\text { ND } \\
0.078 \\
\text { ND } \\
\text { ND } \\
\text { ND } \\
\text { ND } \\
\text { ND } \\
\text { ND } \\
\text { ND } \\
\text { ND }\end{array}$ & $\begin{array}{l}0.030 \\
0.030 \\
0.010 \\
0.030 \\
0.030 \\
0.090 \\
0.030 \\
0.030 \\
0.010 \\
0.030 \\
0.030 \\
0.090 \\
0.030 \\
0.030 \\
0.010 \\
0.030 \\
0.030 \\
0.090 \\
0.030 \\
0.030 \\
0.010 \\
0.030 \\
2.50 \\
0.030 \\
0.090 \\
0.030 \\
0.030 \\
0.010 \\
0.030 \\
0.030 \\
0.090 \\
0.030 \\
0.030 \\
0.010 \\
1.3 \\
1.3 \\
0.40 \\
0.40 \\
3.3 \\
3.3 \\
8.00 \\
2.7 \\
2.7 \\
0.20 \\
0.90 \\
0.90 \\
0.10 \\
0.10 \\
0.050 \\
0.030 \\
2.2 \\
2.2 \\
6.0 \\
6.0 \\
2.50 \\
0.677 \\
0.030 \\
0.090 \\
0.030 \\
0.030 \\
0.010\end{array}$ & 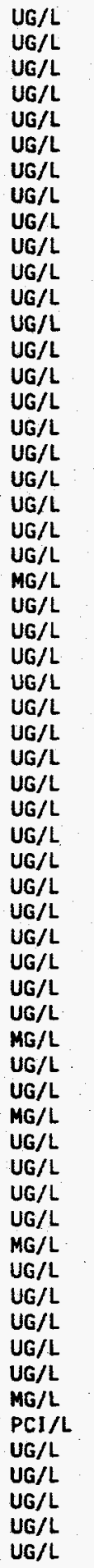 & $\begin{array}{l}\text { USATHAMA } \\
\text { USATHAMA } \\
\text { USATHAMA } \\
\text { USATHAMA } \\
\text { USATHAMA } \\
\text { USATHAMA } \\
\text { USATHAMA } \\
\text { USATHAMA } \\
\text { USATHAMA } \\
\text { USATHAMA } \\
\text { USATHAMA } \\
\text { USATHAMA } \\
\text { USATHAMA } \\
\text { USATHAMA } \\
\text { USATHAMA } \\
\text { USATHAMA } \\
\text { USATHAMA } \\
\text { USATHAMA } \\
\text { USATHAMA } \\
\text { USATHAMA } \\
\text { USATHAMA } \\
\text { USATHAMA } \\
\text { EPA } 300.0 \\
\text { USATHAMA } \\
\text { USATHAMA } \\
\text { USATHAMA } \\
\text { USATHAMA } \\
\text { USATHAMA } \\
\text { USATHAMA } \\
\text { USATHAMA } \\
\text { USATHAMA } \\
\text { USATHAMA } \\
\text { USATHAMA } \\
\text { USATHAMA } \\
\text { EPA CLP } \\
\text { EPA CLP } \\
\text { EPA CLP } \\
\text { EPA CLP } \\
\text { EPA CLP } \\
\text { EPA CLP } \\
\text { EPA } 300.0 \\
\text { EPA CLP } \\
\text { EPA CLP } \\
\text { EPA } 300.0 \\
\text { EPA CLP } \\
\text { EPA CLP } \\
\text { EPA CLP } \\
\text { EPA CLP } \\
\text { EPA } 353.1 \\
\text { USATHAMA } \\
\text { EPA CLP } \\
\text { EPA CLP } \\
\text { EPA CLP } \\
\text { EPA CLP } \\
\text { EPA 300.0 } \\
\text { ASTM } 5174-91 \\
\text { USATHAMA } \\
\text { USATHAMA } \\
\text { USATHAMA } \\
\text { USATHAMA } \\
\text { USATHAMA } \\
\text { SSTH }\end{array}$ & \\
\hline
\end{tabular}




\begin{tabular}{|c|c|c|c|c|c|c|}
\hline WSSRAP_ID & DATE_SAM & PARAMETER & CONC & $D L$ & UNITS & METHOD \\
\hline $\begin{array}{l}G W-1037-0198 \\
G W-1037-0198-F \\
G W-1037-0198 \\
G W-1037-0198-F \\
G W-1037-0198 \\
G W-1037-0198-F \\
G W-1037-0198 \\
G W-1037-0198 \\
G W-1037-0198-F \\
G W-1037-0198 \\
G W-1037-0198 \\
G H-1037-0198-F \\
G W-1037-0198 \\
G W-1037-0198-F \\
G W-1037-0198 \\
G W-1037-0298 \\
G W-1037-0198 \\
G W-1037-0198-F \\
G W-1037-0198 \\
G W-1037-0198-F \\
G W-1037-0198 \\
G W-1037-0198 \\
G W-1038-0198 \\
G W-1039-0198 \\
G W-1040-0298 \\
G W-1040-0298 \\
G W-1040-0298 \\
G W-1040-0298 \\
G W-1040-0298 \\
G W-1040-0198 \\
G W-1040-0198-F \\
G W-1040-0198 \\
G W-1040-0198-F \\
G W-1040-0198 \\
G W-1040-0198-F \\
G W-1040-0198 \\
G W-1040-0198 \\
G W-1040-0198-F \\
G W-1040-0198 \\
G W-1040-0198 \\
G W-1040-0198-F \\
G W-1040-0198 \\
G W-1040-0198-F \\
G W-1040-0198 \\
G W-1040-0298 \\
G W-1040-0198 \\
G W-1040-0198-F \\
G W-1040-0198 \\
G W-1040-0198-F \\
G W-1040-0198 \\
G W-1040-0198 \\
G W-1041-0298 \\
G W-1041-0298 \\
G W-1041-0298 \\
G W-1041-0298 \\
G W-1041-0298 \\
G W-1041-0198 \\
G W-1041-0198-F \\
G W-1041-0198 \\
G W-1041-0198-F \\
G W-1041-0198\end{array}$ & $\begin{array}{l}01 / 27 / 98 \\
01 / 27 / 98 \\
01 / 27 / 98 \\
01 / 27 / 98 \\
01 / 27 / 98 \\
01 / 27 / 98 \\
01 / 27 / 98 \\
01 / 27 / 98 \\
01 / 27 / 98 \\
01 / 27 / 98 \\
01 / 27 / 98 \\
01 / 27 / 98 \\
01 / 27 / 98 \\
01 / 27 / 98 \\
01 / 27 / 98 \\
04 / 21 / 98 \\
01 / 27 / 98 \\
01 / 27 / 98 \\
01 / 27 / 98 \\
01 / 27 / 98 \\
01 / 27 / 98 \\
01 / 27 / 98 \\
01 / 27 / 98 \\
01 / 27 / 98 \\
04 / 22 / 98 \\
04 / 22 / 98 \\
04 / 22 / 98 \\
04 / 22 / 98 \\
04 / 22 / 98 \\
01 / 27 / 98 \\
01 / 27 / 98 \\
01 / 27 / 98 \\
01 / 27 / 98 \\
01 / 27 / 98 \\
01 / 27 / 98 \\
01 / 27 / 98 \\
01 / 27 / 98 \\
01 / 27 / 98 \\
01 / 27 / 98 \\
01 / 27 / 98 \\
01 / 27 / 98 \\
01 / 27 / 98 \\
01 / 27 / 98 \\
01 / 27 / 98 \\
04 / 22 / 98 \\
01 / 27 / 98 \\
01 / 27 / 98 \\
01 / 27 / 98 \\
01 / 27 / 98 \\
01 / 27 / 98 \\
011 / 27 / 98 \\
04 / 22 / 98 \\
04 / 22 / 98 \\
04 / 22 / 98 \\
04 / 22 / 98 \\
04 / 22 / 98 \\
01 / 27 / 98 \\
01 / 27 / 98 \\
01 / 27 / 98 \\
01 / 27 / 98 \\
01 / 27 / 98\end{array}$ & 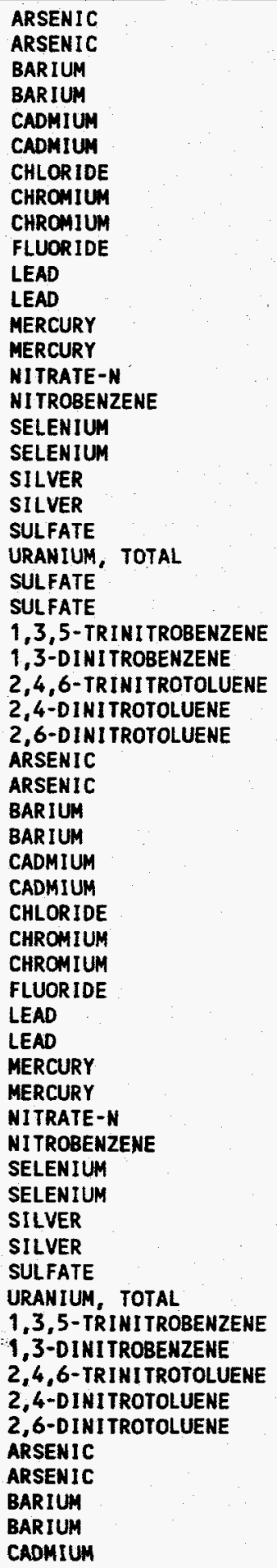 & $\begin{array}{l}12.0 \\
1.9 \\
571 \\
93.2 \\
\text { ND } \\
\text { ND } \\
3.57 \\
41.0 \\
\text { ND } \\
0.39 \\
23.9 \\
\text { ND } \\
\text { ND } \\
\text { ND } \\
0.20 \\
\text { ND } \\
\text { ND } \\
\text { ND } \\
\text { ND } \\
\text { ND } \\
217 \\
1.82 \\
248 \\
145 \\
\text { ND } \\
\text { ND } \\
\text { ND } \\
\text { ND } \\
\text { ND } \\
2.5 \\
\text { ND } \\
205 \\
182 \\
\text { ND } \\
\text { ND } \\
24.2 \\
\text { ND } \\
\text { ND } \\
0.36 \\
\text { ND } \\
\text { ND } \\
\text { ND } \\
\text { ND } \\
\text { ND } \\
\text { ND } \\
\text { ND } \\
\text { ND } \\
\text { ND } \\
\text { ND } \\
266 \\
6.96 \\
\text { ND } \\
\text { ND } \\
\text { ND } \\
\text { ND } \\
1.4 \\
\text { ND } \\
357 \\
360 \\
\text { ND }\end{array}$ & $\begin{array}{l}1.3 \\
1.3 \\
0.40 \\
0.40 \\
3.3 \\
3.3 \\
0.20 \\
2.7 \\
2.7 \\
0.20 \\
0.90 \\
0.90 \\
0.10 \\
0.10 \\
0.050 \\
0.030 \\
2.2 \\
2.2 \\
6.0 \\
6.0 \\
10.0 \\
0.677 \\
10.0 \\
5.00 \\
0.030 \\
0.090 \\
0.030 \\
0.030 \\
0.010 \\
1.3 \\
1.3 \\
0.40 \\
0.40 \\
3.3 \\
3.3 \\
1.00 \\
2.7 \\
2.7 \\
0.20 \\
0.90 \\
0.90 \\
0.10 \\
0.10 \\
0.050 \\
0.030 \\
2.2 \\
2.2 \\
6.0 \\
6.0 \\
10.0 \\
0.677 \\
0.030 \\
0.090 \\
0.030 \\
0.030 \\
0.010 \\
1.3 \\
1.3 \\
0.40 \\
0.40 \\
3.3 \\
\end{array}$ & $\begin{array}{l}U G / L \\
U G / L \\
U G / L \\
U G / L \\
U G / L \\
U G / L \\
M G / L \\
U G / L \\
U G / L \\
M G / L \\
U G / L \\
U G / L \\
U G / L \\
U G / L \\
M G / L \\
U G / L \\
U G / L \\
U G / L \\
U G / L \\
U G / L \\
M G / L \\
P C I / L \\
M G / L \\
M G / L \\
U G / L \\
U G / L \\
U G / L \\
U G / L \\
U G / L \\
U G / L \\
U G / L \\
U G / L \\
U G / L \\
U G / L \\
U G / L \\
M G / L \\
U G / L \\
U G / L \\
M G / L \\
U G / L \\
U G / L \\
U G / L \\
U G / L \\
M G / L \\
U G / L \\
U G / L \\
U G / L \\
U G / L \\
U G / L \\
M G / L \\
P C I / L \\
U G / L \\
U G / L \\
U G / L \\
U G / L \\
U G / L \\
U G / L \\
U G / L \\
U G / L \\
U G / L \\
U G / L \\
U\end{array}$ & $\begin{array}{l}\text { EPA CLP } \\
\text { EPA CLP } \\
\text { EPA CLP } \\
\text { EPA CLP } \\
\text { EPA CLP } \\
\text { EPA CLP } \\
\text { EPA } 300.0 \\
\text { EPA CLP } \\
\text { EPA CLP } \\
\text { EPA } 300.0 \\
\text { EPA CLP } \\
\text { EPA CLP } \\
\text { EPA CLP } \\
\text { EPA CLP } \\
\text { EPA } 353.1 \\
\text { USATHAMA } \\
\text { EPA CLP } \\
\text { EPA CLP } \\
\text { EPA CLP } \\
\text { EPA CLP } \\
\text { EPA } 300.0 \\
\text { ASTM } 5174-91 \\
\text { EPA } 300.0 \\
\text { EPA } 300.0 \\
\text { USATHAMA } \\
\text { USATHAMA } \\
\text { USATHAMA } \\
\text { USATHAMA } \\
\text { USATHAMA } \\
\text { EPA CLP } \\
\text { EPA CLP } \\
\text { EPA CLP } \\
\text { EPA CLP } \\
\text { EPA CLP } \\
\text { EPA CLP } \\
\text { EPA } 300.0 \\
\text { EPA CLP } \\
\text { EPA CLP } \\
\text { EPA } 300.0 \\
\text { EPA CLP } \\
\text { EPA CLP } \\
\text { EPA CLP } \\
\text { EPA CLP } \\
\text { EPA } 353.1 \\
\text { USATHAMA } \\
\text { EPA CLP } \\
\text { EPA CLP } \\
\text { EPA CLP } \\
\text { EPA CLP } \\
\text { EPA } 300.0 \\
\text { ASTM } 5174-91 \\
\text { USATHAMA } \\
\text { USATHAMA } \\
\text { USATHAMA } \\
\text { USATHAMA } \\
\text { USATHAMA } \\
\text { EPA CLP } \\
\text { EPA CLP } \\
\text { EPA CLP } \\
\text { EPA CLP } \\
\text { EPA CLP } \\
\text { EPA }\end{array}$ \\
\hline
\end{tabular}




\begin{tabular}{|c|c|c|c|c|c|c|}
\hline WSSRAP_ID & DATE_SAM & PARAMETER & CONC & DL & UNITS & METHOO \\
\hline 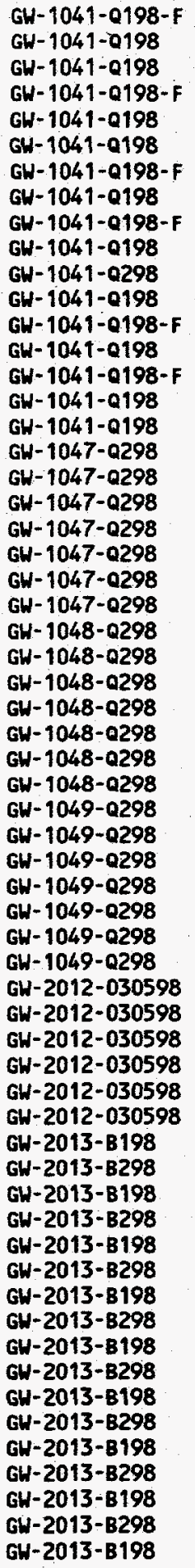 & $\begin{array}{l}01 / 27 / 98 \\
01 / 27 / 98 \\
01 / 27 / 98 \\
01 / 27 / 98 \\
01 / 27 / 98 \\
01 / 27 / 98 \\
01 / 27 / 98 \\
01 / 27 / 98 \\
01 / 27 / 98 \\
01 / 27 / 98 \\
04 / 22 / 98 \\
01 / 27 / 98 \\
01 / 27 / 98 \\
01 / 27 / 98 \\
01 / 27 / 98 \\
01 / 27 / 98 \\
01 / 27 / 98 \\
04 / 29 / 98 \\
04 / 29 / 98 \\
04 / 29 / 98 \\
04 / 29 / 98 \\
04 / 29 / 98 \\
04 / 29 / 98 \\
04 / 29 / 98 \\
04 / 29 / 98 \\
04 / 29 / 98 \\
04 / 29 / 98 \\
04 / 29 / 98 \\
04 / 29 / 98 \\
04 / 29 / 98 \\
04 / 29 / 98 \\
04 / 29 / 98 \\
04 / 29 / 98 \\
04 / 29 / 98 \\
04 / 29 / 98 \\
04 / 29 / 98 \\
04 / 29 / 98 \\
04 / 29 / 98 \\
03 / 05 / 98 \\
03 / 05 / 98 \\
03 / 05 / 98 \\
03 / 05 / 98 \\
03 / 05 / 98 \\
03 / 05 / 98 \\
01 / 29 / 98 \\
03 / 25 / 98 \\
01 / 29 / 98 \\
03 / 25 / 98 \\
01 / 29 / 98 \\
03 / 25 / 98 \\
01 / 29 / 98 \\
03 / 25 / 98 \\
01 / 29 / 98 \\
03 / 25 / 98 \\
01 / 29 / 98 \\
03 / 25 / 98 \\
01 / 29 / 98 \\
03 / 25 / 98 \\
01 / 29 / 98 \\
03 / 25 / 98 \\
01 / 29 / 98\end{array}$ & 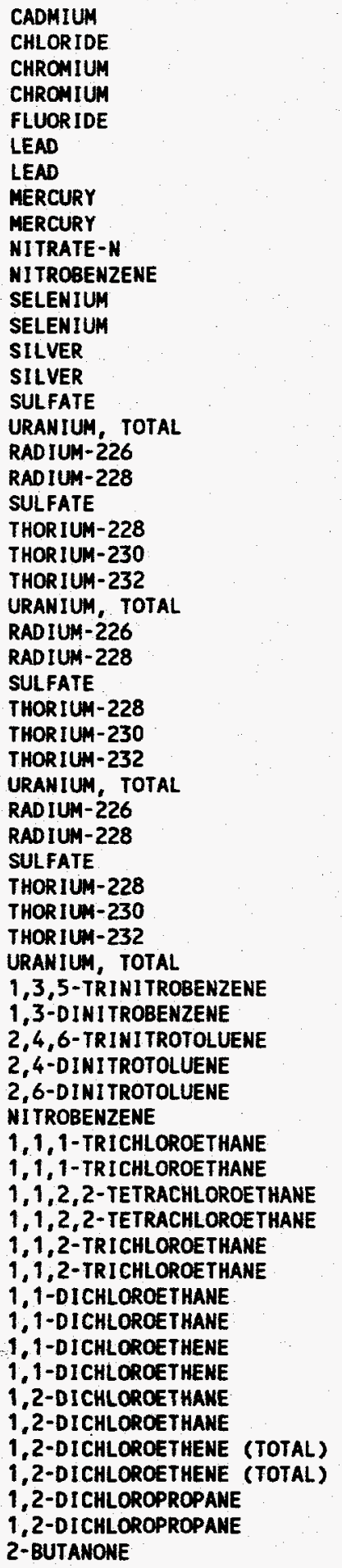 & $\begin{array}{l}\text { ND } \\
5.59 \\
\text { ND } \\
\text { ND } \\
0.24 \\
\text { ND } \\
\text { ND } \\
\text { ND } \\
\text { ND } \\
0.054 \\
\text { ND } \\
\text { ND } \\
\text { ND } \\
\text { ND } \\
\text { ND } \\
50.7 \\
6.01 \\
0.410 \\
0.356 \\
183 \\
0.314 \\
0.219 \\
0.253 \\
3.70 \\
0.426 \\
0.424 \\
75.0 \\
0.150 \\
(0.04 \\
0.100 \\
613 \\
0.486 \\
0.770 \\
19.0 \\
60.01 \\
60.03 \\
(0.00 \\
\text { ND } \\
7.2 \\
\text { ND } \\
25 \\
1.5 \\
8.7 \\
\text { ND } \\
\text { ND } \\
\text { ND } \\
\text { ND } \\
\text { ND } \\
\text { ND } \\
\text { ND } \\
\text { ND } \\
\text { ND } \\
\text { ND } \\
\text { ND } \\
\text { ND } \\
\text { ND } \\
(8) \\
(6) \\
\text { ND } \\
\text { ND } \\
\text { ND }\end{array}$ & $\begin{array}{l}3.3 \\
1.00 \\
2.7 \\
2.7 \\
0.20 \\
0.90 \\
0.90 \\
0.10 \\
0.10 \\
0.050 \\
0.030 \\
2.2 \\
2.2 \\
6.0 \\
6.0 \\
2.50 \\
0.677 \\
0.110 \\
0.243 \\
5.00 \\
0.099 \\
0.071 \\
0.071 \\
0.677 \\
0.100 \\
0.230 \\
5.00 \\
0.107 \\
0.078 \\
0.064 \\
6.77 \\
0.127 \\
0.275 \\
1.00 \\
0.081 \\
0.160 \\
0.086 \\
0.677 \\
0.030 \\
0.090 \\
0.030 \\
0.030 \\
0.010 \\
0.030 \\
10 \\
10 \\
10 \\
10 \\
10 \\
10 \\
10 \\
10 \\
10 \\
10 \\
10 \\
10 \\
10 \\
10 \\
10 \\
10 \\
10 \\
\end{array}$ & 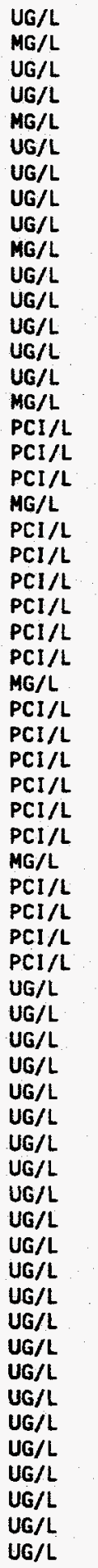 & $\begin{array}{l}\text { EPA CLP } \\
\text { EPA } 300: 0 \\
\text { EPA CLP } \\
\text { EPA CLP } \\
\text { EPA } 300.0 \\
\text { EPA CLP } \\
\text { EPA CLP } \\
\text { EPA CLP } \\
\text { EPA CLP } \\
\text { EPA } 353.1 \\
\text { USATHAMA } \\
\text { EPA CLP } \\
\text { EPA CLP } \\
\text { EPA CLP } \\
\text { EPA CLP } \\
\text { EPA } 300.0 \\
\text { ASTM } 5174-91 \\
\text { EPA } 904.0 \\
\text { EPA } 904.0 \\
\text { EPA } 300.0 \\
\text { NAS-NS-3004 } \\
\text { NAS-NS-3004 } \\
\text { NAS-NS-3004 } \\
\text { ASTM } 5174-91 \\
\text { EPA } 904.0 \\
\text { EPA. } 904.0 \\
\text { EPA } 300.0 \\
\text { NAS-NS-3004 } \\
\text { NAS-NS-3004 } \\
\text { NAS-NS-3004 } \\
\text { ASTM } 5174-91 \\
\text { EPA } 904.0 \\
\text { EPA } 904.0 \\
\text { EPA } 300.0 \\
\text { NAS-NS-3004 } \\
\text { NAS-NS-3004 } \\
\text { NAS-NS-3004 } \\
\text { ASTM } 5174-91 \\
\text { USATHAMA } \\
\text { USATHAMA } \\
\text { USATHAMA } \\
\text { USATHAMA } \\
\text { USATHAMA } \\
\text { USATHAMA } \\
\text { EPA CLP } \\
\text { EPA CLP } \\
\text { EPA CLP } \\
\text { EPA CLP } \\
\text { EPA CLP } \\
\text { EPA CLP } \\
\text { EPA CLP } \\
\text { EPA CLP } \\
\text { EPA CLP } \\
\text { EPA CLP } \\
\text { EPA CLP } \\
\text { EPA CLP } \\
\text { EPA CLP } \\
\text { EPA CLP } \\
\text { EPA CLP } \\
\text { EPA CLP } \\
\text { EPA CLP } \\
\text { EPA }\end{array}$ \\
\hline
\end{tabular}




\begin{tabular}{|c|c|c|c|c|c|c|}
\hline WSSRAP_ID & DATE_SAM & PARAMETER & CONC & DL & UNITS & METHOD \\
\hline 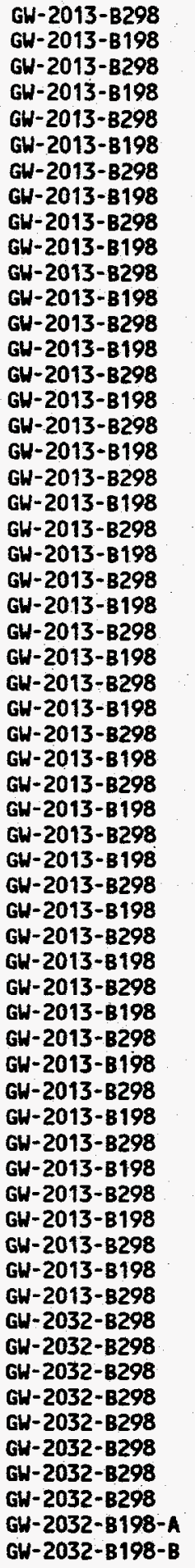 & $\begin{array}{l}03 / 25 / 98 \\
01 / 29 / 98 \\
03 / 25 / 98 \\
01 / 29 / 98 \\
03 / 25 / 98 \\
01 / 29 / 98 \\
03 / 25 / 98 \\
01 / 29 / 98 \\
03 / 25 / 98 \\
01 / 29 / 98 \\
03 / 25 / 98 \\
01 / 29 / 98 \\
03 / 25 / 98 \\
01 / 29 / 98 \\
03 / 25 / 98 \\
01 / 29 / 98 \\
03 / 25 / 98 \\
01 / 29 / 98 \\
03 / 25 / 98 \\
01 / 29 / 98 \\
03 / 25 / 98 \\
01 / 29 / 98 \\
03 / 25 / 98 \\
01 / 29 / 98 \\
03 / 25 / 98 \\
01 / 29 / 98 \\
03 / 25 / 98 \\
01 / 29 / 98 \\
03 / 25 / 98 \\
01 / 29 / 98 \\
03 / 25 / 98 \\
01 / 29 / 98 \\
03 / 25 / 98 \\
01 / 29 / 98 \\
03 / 25 / 98 \\
01 / 29 / 98 \\
03 / 25 / 98 \\
01 / 29 / 98 \\
03 / 25 / 98 \\
01 / 29 / 98 \\
03 / 25 / 98 \\
01 / 29 / 98 \\
03 / 25 / 98 \\
01 / 29 / 98 \\
03 / 25 / 98 \\
01 / 29 / 98 \\
03 / 25 / 98 \\
01 / 29 / 98 \\
03 / 25 / 98 \\
01 / 29 / 98 \\
03 / 25 / 98 \\
03 / 17 / 98 \\
03 / 17 / 98 \\
03 / 17 / 98 \\
03 / 17 / 98 \\
03 / 17 / 98 \\
03 / 17 / 98 \\
03 / 17 / 98 \\
03 / 17 / 98 \\
01 / 19 / 98 \\
01 / 21 / 98\end{array}$ & 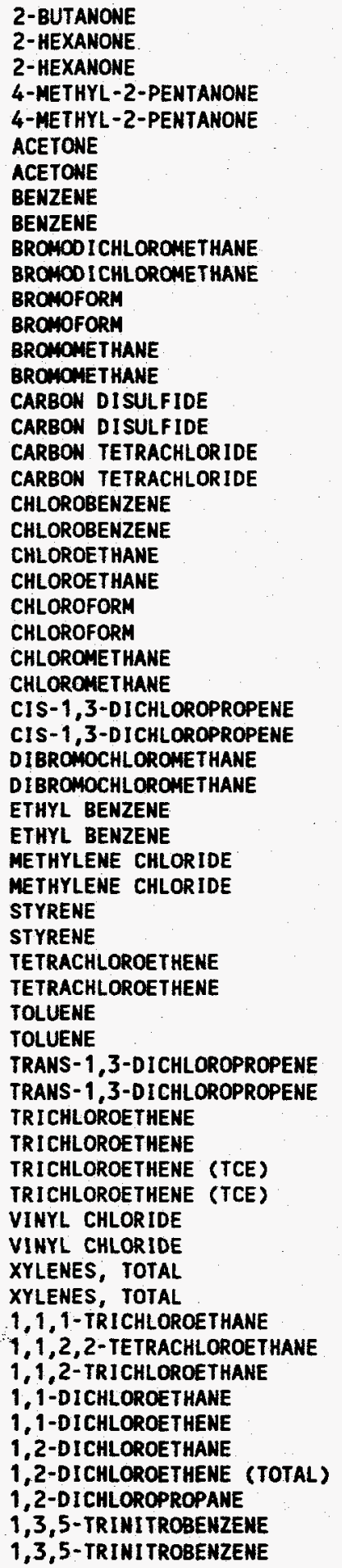 & 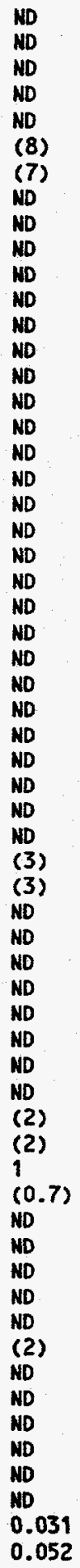 & $\begin{array}{l}10 \\
10 \\
10 \\
10 \\
10 \\
10 \\
10 \\
10 \\
10 \\
10 \\
10 \\
10 \\
10 \\
10 \\
10 \\
10 \\
10 \\
10 \\
10 \\
10 \\
10 \\
10 \\
10 \\
10 \\
10 \\
10 \\
10 \\
10 \\
10 \\
10 \\
10 \\
10 \\
10 \\
10 \\
10 \\
10 \\
10 \\
10 \\
10 \\
10 \\
10 \\
10 \\
10 \\
10 \\
10 \\
1 \\
1 \\
10 \\
10 \\
10 \\
10 \\
10 \\
10 \\
10 \\
10 \\
10 \\
10 \\
10 \\
10 \\
0.030 \\
0.030\end{array}$ & $\begin{array}{l}U G / L \\
U G / L \\
U G / L \\
U G / L \\
U G / L \\
U G / L \\
U G / L \\
U G / L \\
U G / L \\
U G / L \\
U G / L \\
U G / L \\
U G / L \\
U G / L \\
U G / L \\
U G / L \\
U G / L \\
U G / L \\
U G / L \\
U G / L \\
U G / L \\
U G / L \\
U G / L \\
U G / L \\
U G / L \\
U G / L \\
U G / L \\
U G / L \\
U G / L \\
U G / L \\
U G / L \\
U G / L \\
U G / L \\
U G / L \\
U G / L \\
U G / L \\
U G / L \\
U G / L \\
U G / L \\
U G / L \\
U G / L \\
U G / L \\
U G / L \\
U G / L \\
U G / L \\
U G / L \\
U G / L \\
U G / L \\
U G / L \\
U G / L \\
U G / L \\
U G / L \\
U G / L \\
U G / L \\
U G / L \\
U G / L \\
U G / L \\
U G / L \\
U G / L \\
U G / L \\
U G / L \\
U\end{array}$ & $\begin{array}{l}\text { EPA CLP } \\
\text { EPA CLP } \\
\text { EPA CLP } \\
\text { EPA CLP } \\
\text { EPA CLP } \\
\text { EPA CLP } \\
\text { EPA CLP } \\
\text { EPA CLP } \\
\text { EPA CLP } \\
\text { EPA CLP } \\
\text { EPA CLP } \\
\text { EPA CLP } \\
\text { EPA CLP } \\
\text { EPA CLP } \\
\text { EPA CLP } \\
\text { EPA CLP } \\
\text { EPA CLP } \\
\text { EPA CLP } \\
\text { EPA CLP } \\
\text { EPA CLP } \\
\text { EPA CLP } \\
\text { EPA CLP } \\
\text { EPA CLP } \\
\text { EPA CLP } \\
\text { EPA CLP } \\
\text { EPA CLP } \\
\text { EPA CLP } \\
\text { EPA CLP } \\
\text { EPA CLP } \\
\text { EPA CLP } \\
\text { EPA CLP } \\
\text { EPA CLP } \\
\text { EPA CLP } \\
\text { EPA CLP } \\
\text { EPA CLP } \\
\text { EPA CLP } \\
\text { EPA CLP } \\
\text { EPA CLP } \\
\text { EPA CLP } \\
\text { EPA CLP } \\
\text { EPA CLP } \\
\text { EPA CLP } \\
\text { EPA CLP } \\
\text { EPA CLP } \\
\text { EPA CLP } \\
\text { EPA } 8260 \\
\text { EPA } 8260 \\
\text { EPA CLP } \\
\text { EPA CLP } \\
\text { EPA CLP } \\
\text { EPA CLP } \\
\text { EPA CLP } \\
\text { EPA CLP } \\
\text { EPA CLP } \\
\text { EPA CLP } \\
\text { EPA CLP } \\
\text { EPA CLP } \\
\text { EPA CLP } \\
\text { EPA CLP } \\
\text { USATHAMA } \\
\text { USATHAMA }\end{array}$ \\
\hline
\end{tabular}




\begin{tabular}{|c|c|c|c|c|c|c|}
\hline WSSRAP_ID & DATE_SAM & PARAMETER & CONC & DL & UNITS & METHOD \\
\hline 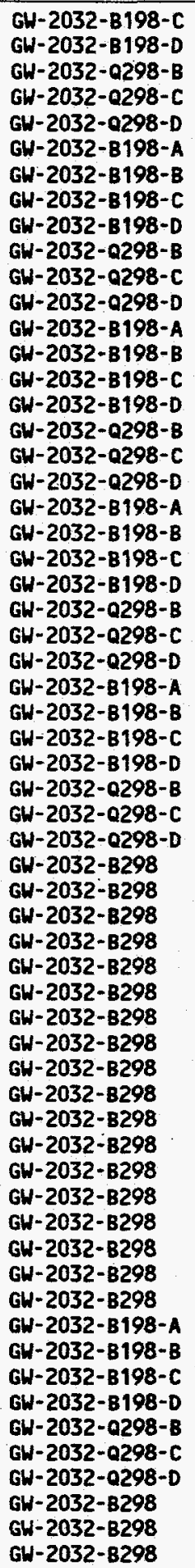 & $\begin{array}{l}01 / 22 / 98 \\
01 / 23 / 98 \\
04 / 08 / 98 \\
04 / 09 / 98 \\
04 / 10 / 98 \\
01 / 19 / 98 \\
01 / 21 / 98 \\
01 / 22 / 98 \\
01 / 23 / 98 \\
04 / 08 / 98 \\
04 / 09 / 98 \\
04 / 10 / 98 \\
01 / 19 / 98 \\
01 / 21 / 98 \\
01 / 22 / 98 \\
01 / 23 / 98 \\
04 / 08 / 98 \\
04 / 09 / 98 \\
04 / 10 / 98 \\
01 / 19 / 98 \\
01 / 21 / 98 \\
01 / 22 / 98 \\
01 / 23 / 98 \\
04 / 08 / 98 \\
04 / 09 / 98 \\
04 / 10 / 98 \\
01 / 19 / 98 \\
01 / 21 / 98 \\
01 / 22 / 98 \\
01 / 23 / 98 \\
04 / 08 / 98 \\
04 / 09 / 98 \\
04 / 10 / 98 \\
03 / 17 / 98 \\
03 / 17 / 98 \\
03 / 17 / 98 \\
03 / 17 / 98 \\
03 / 17 / 98 \\
03 / 17 / 98 \\
03 / 17 / 98 \\
03 / 17 / 98 \\
03 / 17 / 98 \\
03 / 17 / 98 \\
03 / 17 / 98 \\
03 / 17 / 98 \\
03 / 17 / 98 \\
03 / 17 / 98 \\
03 / 17 / 98 \\
03 / 17 / 98 \\
03 / 17 / 98 \\
03 / 17 / 98 \\
01 / 19 / 98 \\
01 / 21 / 98 \\
01 / 22 / 98 \\
01 / 23 / 98 \\
04 / 08 / 98 \\
04 / 09 / 98 \\
04 / 10 / 98 \\
03 / 17 / 98 \\
03 / 17 / 98 \\
03 / 17 / 98\end{array}$ & 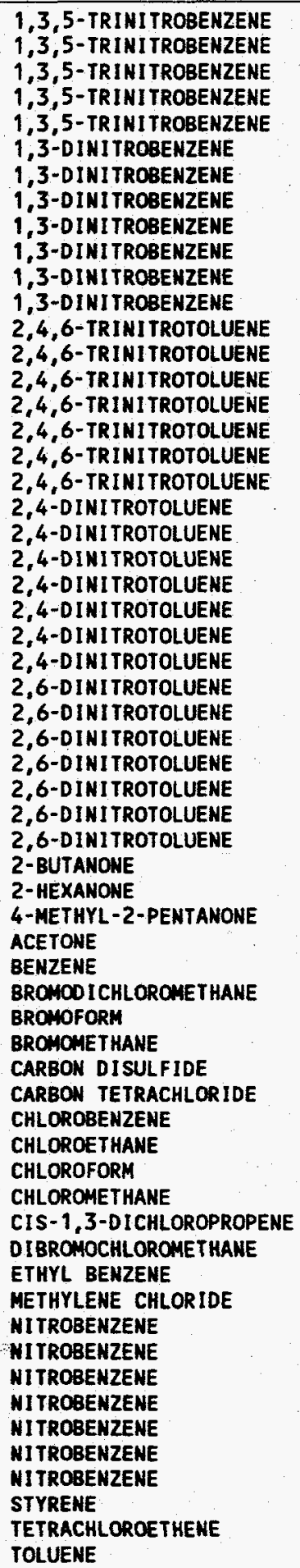 & $\begin{array}{l}0.057 \\
0.051 \\
0.094 \\
0.085 \\
0.086 \\
\text { ND } \\
\text { ND } \\
\text { ND } \\
\text { ND } \\
\text { ND } \\
\text { ND } \\
0.061 \\
0.16 \\
0.17 \\
0.15 \\
0.24 \\
0.23 \\
0.24 \\
\text { Co. } \\
0.043 \\
0.043 \\
0.039 \\
0.073 \\
0.062 \\
0.065 \\
0.061 \\
0.15 \\
0.14 \\
0.13 \\
0.21 \\
0.18 \\
0.19 \\
\text { ND } \\
\text { ND } \\
\text { ND } \\
11 \\
\text { ND } \\
\text { ND } \\
(2) \\
\text { ND } \\
\text { ND } \\
\text { ND } \\
\text { ND } \\
\text { ND } \\
\text { ND } \\
\text { ND } \\
\text { ND } \\
(1) \\
\text { ND } \\
\text { NB } \\
\text { ND } \\
\text { ND } \\
\text { ND } \\
\text { ND } \\
\text { ND } \\
\text { ND } \\
\text { ND } \\
\text { ND } \\
\text { ND }\end{array}$ & $\begin{array}{l}0.030 \\
0.030 \\
0.030 \\
0.030 \\
0.030 \\
0.090 \\
0.090 \\
0.090 \\
0.090 \\
0.090 \\
0.090 \\
0.090 \\
0.030 \\
0.030 \\
0.030 \\
0.030 \\
0.030 \\
0.030 \\
0.030 \\
0.030 \\
0.030 \\
0.030 \\
0.030 \\
0.030 \\
0.030 \\
0.030 \\
0.010 \\
0.010 \\
0.010 \\
0.010 \\
0.010 \\
0.010 \\
0.010 \\
10 \\
10 \\
10 \\
10 \\
10 \\
10 \\
10 \\
10 \\
10 \\
10 \\
10 \\
10 \\
10 \\
10 \\
10 \\
10 \\
10 \\
10 \\
0.030 \\
0.030 \\
0.030 \\
0.030 \\
0.030 \\
0.030 \\
0.030 \\
10 \\
10 \\
10 \\
0\end{array}$ & $\begin{array}{l}\text { UG/L } \\
U G / L \\
U G / L \\
U G / L \\
U G / L \\
U G / L \\
U G / L \\
U G / L \\
U G / L \\
U G / L \\
U G / L \\
U G / L \\
U G / L \\
U G / L \\
U G / L \\
U G / L \\
U G / L \\
U G / L \\
U G / L \\
U G / L \\
U G / L \\
U G / L \\
U G / L \\
U G / L \\
U G / L \\
U G / L \\
U G / L \\
U G / L \\
U G / L \\
U G / L \\
U G / L \\
U G / L \\
U G / L \\
U G / L \\
U G / L \\
U G / L \\
U G / L \\
U G / L \\
U G / L \\
U G / L \\
U G / L \\
U G / L \\
U G / L \\
U G / L \\
U G / L \\
U G / L \\
U G / L \\
U G / L \\
U G / L \\
U G / L \\
U G / L \\
U G / L \\
U G / L \\
U G / L \\
U G / L \\
U G / L \\
U G / L \\
U G / L \\
U G / L \\
U G / L \\
U G / L\end{array}$ & $\begin{array}{l}\text { USATHAMA } \\
\text { USATHAMA } \\
\text { USATHAMA } \\
\text { USATHAMA } \\
\text { USATHAMA } \\
\text { USATHAMA } \\
\text { USATHAMA } \\
\text { USATHAMA } \\
\text { USATHAMA } \\
\text { USATHAMA } \\
\text { USATHAMA } \\
\text { USATHAMA } \\
\text { USATHAMA } \\
\text { USATHAMA } \\
\text { USATHAMA } \\
\text { USATHAMA } \\
\text { USATHAMA } \\
\text { USATHAMA } \\
\text { USATHAMA } \\
\text { USATHAMA } \\
\text { USATHAMA } \\
\text { USATHAMA } \\
\text { USATHAMA } \\
\text { USATHAMA } \\
\text { USATHAMA } \\
\text { USATHAMA } \\
\text { USATHAMA } \\
\text { USATHAMA } \\
\text { USATHAMA } \\
\text { USATHAMA } \\
\text { USATHAMA } \\
\text { USATHAMA } \\
\text { USATHAMA } \\
\text { EPA CLP } \\
\text { EPA CLP } \\
\text { EPA CLP } \\
\text { EPA CLP } \\
\text { EPA CLP } \\
\text { EPA CLP } \\
\text { EPA CLP } \\
\text { EPA CLP } \\
\text { EPA CLP } \\
\text { EPA CLP } \\
\text { EPA CLP } \\
\text { EPA CLP } \\
\text { EPA CLP } \\
\text { EPA CLP } \\
\text { EPA CLP } \\
\text { EPA CLP } \\
\text { EPA CLP } \\
\text { EPA CLP } \\
\text { USATHAMA } \\
\text { USATHAMA } \\
\text { USATHAMA } \\
\text { USATHAMA } \\
\text { USATHAMA } \\
\text { USATHAMA } \\
\text { USATHAMA } \\
\text { EPA CLP } \\
\text { EPA CLP } \\
\text { EPA }\end{array}$ \\
\hline
\end{tabular}




\begin{tabular}{|c|c|c|c|c|c|c|}
\hline WSSRAP_ID & DATE_SAM & PARAMETER & CONC & DL & UNITS & METHOD \\
\hline 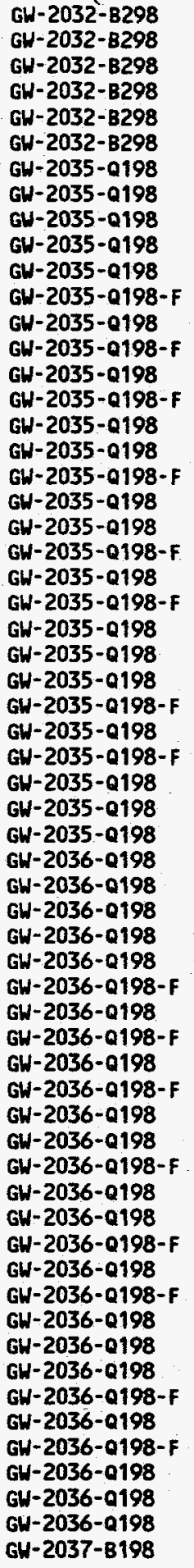 & $\begin{array}{l}03 / 17 / 98 \\
03 / 17 / 98 \\
03 / 17 / 98 \\
03 / 17 / 98 \\
03 / 17 / 98 \\
03 / 17 / 98 \\
02 / 16 / 98 \\
02 / 16 / 98 \\
02 / 16 / 98 \\
02 / 16 / 98 \\
02 / 16 / 98 \\
02 / 16 / 98 \\
02 / 16 / 98 \\
02 / 16 / 98 \\
02 / 16 / 98 \\
02 / 16 / 98 \\
02 / 16 / 98 \\
02 / 16 / 98 \\
02 / 16 / 98 \\
02 / 16 / 98 \\
02 / 16 / 98 \\
02 / 16 / 98 \\
02 / 16 / 98 \\
02 / 16 / 98 \\
02 / 16 / 98 \\
02 / 16 / 98 \\
02 / 16 / 98 \\
02 / 16 / 98 \\
02 / 16 / 98 \\
02 / 16 / 98 \\
02 / 16 / 98 \\
02 / 16 / 98 \\
02 / 16 / 98 \\
02 / 16 / 98 \\
02 / 16 / 98 \\
02 / 16 / 98 \\
02 / 16 / 98 \\
02 / 16 / 98 \\
02 / 16 / 98 \\
02 / 16 / 98 \\
02 / 16 / 98 \\
02 / 16 / 98 \\
02 / 16 / 98 \\
02 / 16 / 98 \\
02 / 16 / 98 \\
02 / 16 / 98 \\
02 / 16 / 98 \\
02 / 16 / 98 \\
02 / 16 / 98 \\
02 / 16 / 98 \\
02 / 16 / 98 \\
02 / 16 / 98 \\
02 / 16 / 98 \\
02 / 16 / 98 \\
02 / 16 / 98 \\
02 / 16 / 98 \\
02 / 16 / 98 \\
02 / 16 / 98 \\
02 / 16 / 98 \\
02 / 16 / 98 \\
02 / 17 / 98\end{array}$ & 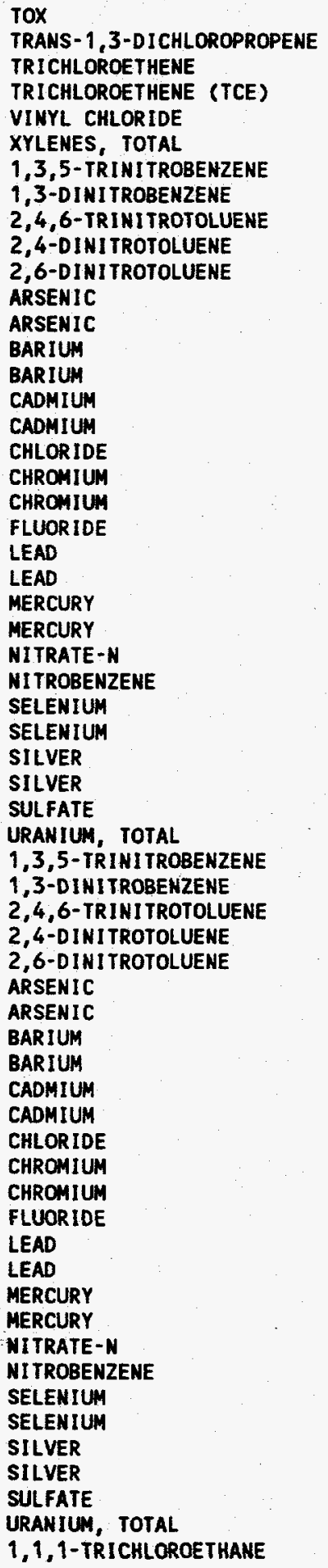 & 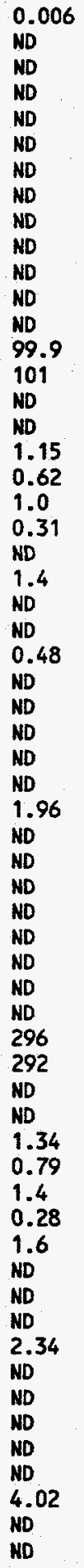 & $\begin{array}{l}0.0050 \\
10 \\
10 \\
1 \\
10 \\
10 \\
10 \\
0.030 \\
0.090 \\
0.030 \\
0 \\
0.030 \\
0.010 \\
1.3 \\
1.3 \\
\mid \\
0.40 \\
0\end{array}$ & $\begin{array}{l}M G / L \\
U G / L \\
U G / L \\
U G / L \\
U G / L \\
U G / L \\
U G / L \\
U G / L \\
U G / L \\
U G / L \\
U G / L \\
U G / L \\
U G / L \\
U G / L \\
U G / L \\
U G / L \\
U G / L \\
M G / L \\
U G / L \\
U G / L \\
M G / L \\
U G / L \\
U G / L \\
U G / L \\
U G / L \\
M G / L \\
U G / L \\
U G / L \\
U G / L \\
U G / L \\
U G / L \\
M G / L \\
P C I / L \\
U G / L \\
U G / L \\
U G / L \\
U G / L \\
U G / L \\
U G / L \\
U G / L \\
U G / L \\
U G / L \\
U G / L \\
U G / L \\
U G / L \\
U G / L \\
U G / L \\
U G / L \\
U G / L \\
U G / L \\
U G / L \\
U G / L \\
U G / L \\
U G / L \\
U G / L \\
U G \\
U G \\
U G \\
U G\end{array}$ & $\begin{array}{l}\text { EPA } 9020 \\
\text { EPA CLP } \\
\text { EPA CLP } \\
\text { EPA } 8260 \\
\text { EPA CLP } \\
\text { EPA CLP } \\
\text { USATHAMA } \\
\text { USATHAMA } \\
\text { USATHAMA } \\
\text { USATHAMA } \\
\text { USATHAMA } \\
\text { EPA CLP } \\
\text { EPA CLP } \\
\text { EPA CLP } \\
\text { EPA CLP } \\
\text { EPA CLP } \\
\text { EPA CLP } \\
\text { EPA } 300.0 \\
\text { EPA CLP } \\
\text { EPA CLP } \\
\text { EPA } 300.0 \\
\text { EPA CLP } \\
\text { EPA CLP } \\
\text { EPA CLP } \\
\text { EPA CLP } \\
\text { EPA } 353.1 \\
\text { USATHAMA } \\
\text { EPA CLP } \\
\text { EPA CLP } \\
\text { EPA CLP } \\
\text { EPA CLP } \\
\text { EPA } 300.0 \\
\text { ASTM } 5174-91 \\
\text { USATHAMA } \\
\text { USATHAMA } \\
\text { USATHAMA } \\
\text { USATHAMA } \\
\text { USATHAMA } \\
\text { EPA CLP } \\
\text { EPA CLP } \\
\text { EPA CLP } \\
\text { EPA CLP } \\
\text { EPA CLP } \\
\text { EPA CLP } \\
\text { EPA } 300.0 \\
\text { EPA CLP } \\
\text { EPA CLP } \\
\text { EPA } 300.0 \\
\text { EPA CLP } \\
\text { EPA CLP } \\
\text { EPA CLP } \\
\text { EPA CLP } \\
\text { EPA } 353.1 \\
\text { USATHAMA } \\
\text { EPA CLP } \\
\text { EPA CLP } \\
\text { EPA CLP } \\
\text { EPA CLP } \\
\text { EPA } 300.0 \\
\text { ASTM } 5174-91 \\
\text { EPA CLP } \\
\end{array}$ \\
\hline
\end{tabular}




\begin{tabular}{|c|c|c|c|c|c|c|}
\hline WSSRAP_ID & DATE_SAM & PARAMETER & CONC & DL & UNITS & METHOD \\
\hline 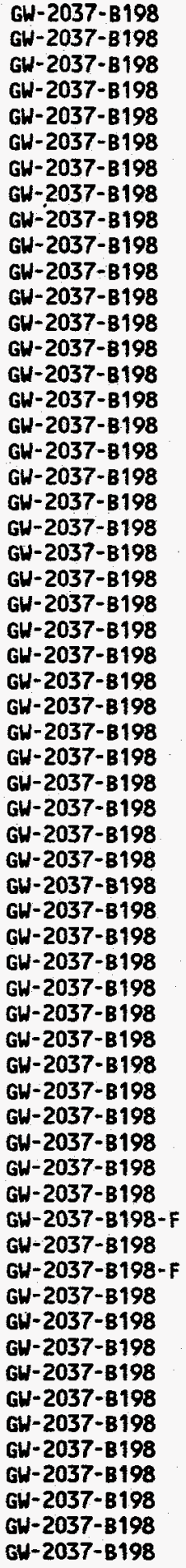 & $\begin{array}{l}02 / 17 / 98 \\
02 / 17 / 98 \\
02 / 17 / 98 \\
02 / 17 / 98 \\
02 / 17 / 98 \\
02 / 17 / 98 \\
02 / 17 / 98 \\
02 / 17 / 98 \\
02 / 17 / 98 \\
02 / 17 / 98 \\
02 / 17 / 98 \\
02 / 17 / 98 \\
02 / 17 / 98 \\
02 / 17 / 98 \\
02 / 17 / 98 \\
02 / 17 / 98 \\
02 / 17 / 98 \\
02 / 17 / 98 \\
02 / 17 / 98 \\
02 / 17 / 98 \\
02 / 17 / 98 \\
02 / 17 / 98 \\
02 / 17 / 98 \\
02 / 17 / 98 \\
02 / 17 / 98 \\
02 / 17 / 98 \\
02 / 17 / 98 \\
02 / 17 / 98 \\
02 / 17 / 98 \\
02 / 17 / 98 \\
02 / 17 / 98 \\
02 / 17 / 98 \\
02 / 17 / 98 \\
02 / 17 / 98 \\
02 / 17 / 98 \\
02 / 17 / 98 \\
02 / 17 / 98 \\
02 / 17 / 98 \\
02 / 17 / 98 \\
02 / 17 / 98 \\
02 / 17 / 98 \\
02 / 17 / 98 \\
02 / 17 / 98 \\
02 / 17 / 98 \\
02 / 17 / 98 \\
02 / 17 / 98 \\
02 / 17 / 98 \\
02 / 17 / 98 \\
02 / 17 / 98 \\
02 / 17 / 98 \\
02 / 17 / 98 \\
02 / 17 / 98 \\
02 / 17 / 98 \\
02 / 17 / 98 \\
02 / 17 / 98 \\
02 / 17 / 98 \\
02 / 17 / 98 \\
02 / 17 / 98 \\
02 / 17 / 98 \\
02 / 17 / 98 \\
02 / 17 / 98\end{array}$ & 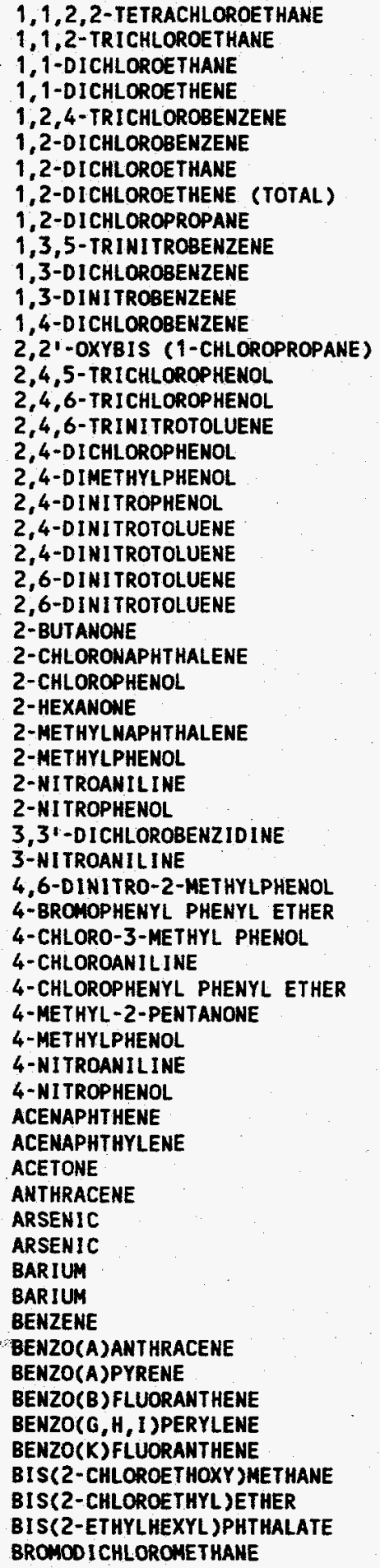 & $\begin{array}{l}\text { ND } \\
\text { ND } \\
\text { ND } \\
\text { ND } \\
\text { ND } \\
\text { ND } \\
\text { C18) } \\
\text { ND } \\
\text { O.20 } \\
\text { ND } \\
\text { C.08 } \\
\text { ND } \\
\text { ND } \\
\text { ND } \\
\text { ND } \\
\text { ND } \\
\text { ND } \\
\text { ND } \\
\text { ND } \\
\text { ND } \\
0.69 \\
\text { ND } \\
\text { O. } 13 \\
\text { ND } \\
\text { ND } \\
\text { ND } \\
\text { ND } \\
\text { ND } \\
\text { ND } \\
\text { ND } \\
\text { ND } \\
\text { ND } \\
\text { ND } \\
\text { ND } \\
\text { ND } \\
\text { ND } \\
\text { ND } \\
\text { ND } \\
\text { ND } \\
\text { ND } \\
\text { ND } \\
\text { ND } \\
\text { ND } \\
\text { ND } \\
\text { 470 } \\
\text { ND } \\
\text { ND } \\
\text { ND } \\
87.0 \\
92.1 \\
\text { ND } \\
\text { ND } \\
\text { ND } \\
\text { ND } \\
\text { ND } \\
\text { ND } \\
\text { ND } \\
\text { ND } \\
\text { ND } \\
\text { ND }\end{array}$ & $\begin{array}{l}100 \\
100 \\
100 \\
100 \\
10 \\
10 \\
100 \\
100 \\
100 \\
0.030 \\
10 \\
0.090 \\
10 \\
10 \\
10 \\
10 \\
0.030 \\
10 \\
10 \\
50 \\
10 \\
0.030 \\
10 \\
0.010 \\
100 \\
10 \\
10 \\
100 \\
10 \\
10 \\
50 \\
10 \\
10 \\
50 \\
50 \\
10 \\
10 \\
10 \\
10 \\
100 \\
10 \\
50 \\
50 \\
10 \\
10 \\
100 \\
10 \\
1.3 \\
1.3 \\
0.40 \\
0.40 \\
100 \\
10 \\
10 \\
10 \\
10 \\
10 \\
10 \\
10 \\
10 \\
100 \\
\\
0 \\
0\end{array}$ & $\begin{array}{l}U G / L \\
U G / L \\
U G / L \\
U G / L \\
U G / L \\
U G / L \\
U G / L \\
U G / L \\
U G / L \\
U G / L \\
U G / L \\
U G / L \\
U G / L \\
U G / L \\
U G / L \\
U G / L \\
U G / L \\
U G / L \\
U G / L \\
U G / L \\
U G / L \\
U G / L \\
U G / L \\
U G / L \\
U G / L \\
U G / L \\
U G / L \\
U G / L \\
U G / L \\
U G / L \\
U G / L \\
U G / L \\
U G / L \\
U G / L \\
U G / L \\
U G / L \\
U G / L \\
U G / L \\
U G / L \\
U G / L \\
U G / L \\
U G / L \\
U G / L \\
U G / L \\
U G / L \\
U G / L \\
U G / L \\
U G / L \\
U G / L \\
U G / L \\
U G / L \\
U G / L \\
U G / L \\
U G / L \\
U G / L \\
U G / L \\
U G / L \\
U G / L \\
U G / L \\
U G / L \\
U G / L \\
\text { U. } \\
U\end{array}$ & $\begin{array}{l}\text { EPA CLP } \\
\text { EPA CLP } \\
\text { EPA CLP } \\
\text { EPA CLP } \\
\text { EPA CLP } \\
\text { EPA CLP } \\
\text { EPA CLP } \\
\text { EPA CLP } \\
\text { EPA CLP } \\
\text { USATHAMA } \\
\text { EPA CLP } \\
\text { USATHAMA } \\
\text { EPA CLP } \\
\text { EPA CLP } \\
\text { EPA CLP } \\
\text { EPA CLP } \\
\text { USATHAMA } \\
\text { EPA CLP } \\
\text { EPA CLP } \\
\text { EPA CLP } \\
\text { EPA CLP } \\
\text { USATHAMA } \\
\text { EPA CLP } \\
\text { USATHAMA } \\
\text { EPA CLP } \\
\text { EPA CLP } \\
\text { EPA CLP } \\
\text { EPA CLP } \\
\text { EPA CLP } \\
\text { EPA CLP } \\
\text { EPA CLP } \\
\text { EPA CLP } \\
\text { EPA CLP } \\
\text { EPA CLP } \\
\text { EPA CLP } \\
\text { EPA CLP } \\
\text { EPA CLP } \\
\text { EPA CLP } \\
\text { EPA CLP } \\
\text { EPA CLP } \\
\text { EPA CLP } \\
\text { EPA CLP } \\
\text { EPA CLP } \\
\text { EPA CLP } \\
\text { EPA CLP } \\
\text { EPA CLP } \\
\text { EPA CLP } \\
\text { EPA CLP } \\
\text { EPA CLP } \\
\text { EPA CLP } \\
\text { EPA CLP } \\
\text { EPA CLP } \\
\text { EPA CLP } \\
\text { EPA CLP } \\
\text { EPA CLP } \\
\text { EPA CLP } \\
\text { EPA CLP } \\
\text { EPA CLP } \\
\text { EPA CLP } \\
\text { EPA CLP } \\
\text { EPA CLP }\end{array}$ \\
\hline
\end{tabular}




\begin{tabular}{|c|c|c|c|c|c|c|}
\hline USSRAP_ID & DATE_SAM & PARAMETER & CONC & DL & UNITS & METHOD \\
\hline 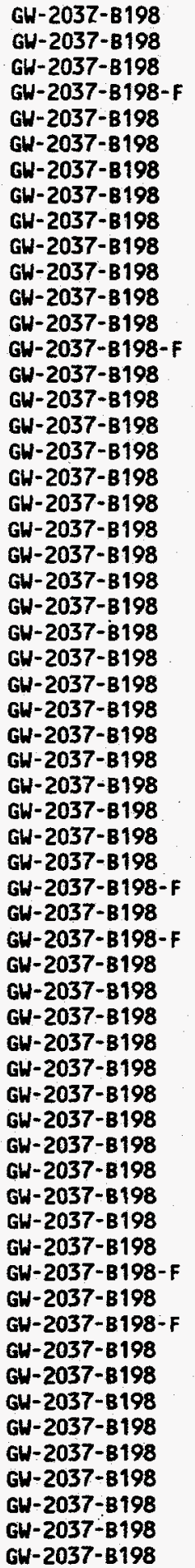 & $\begin{array}{l}02 / 17 / 98 \\
02 / 17 / 98 \\
02 / 17 / 98 \\
02 / 17 / 98 \\
02 / 17 / 98 \\
02 / 17 / 98 \\
02 / 17 / 98 \\
02 / 17 / 98 \\
02 / 17 / 98 \\
02 / 17 / 98 \\
02 / 17 / 98 \\
02 / 17 / 98 \\
02 / 17 / 98 \\
02 / 17 / 98 \\
02 / 17 / 98 \\
02 / 17 / 98 \\
02 / 17 / 98 \\
02 / 17 / 98 \\
02 / 17 / 98 \\
02 / 17 / 98 \\
02 / 17 / 98 \\
02 / 17 / 98 \\
02 / 17 / 98 \\
02 / 17 / 98 \\
02 / 17 / 98 \\
02 / 17 / 98 \\
02 / 17 / 98 \\
02 / 17 / 98 \\
02 / 17 / 98 \\
02 / 17 / 98 \\
02 / 17 / 98 \\
02 / 17 / 98 \\
02 / 17 / 98 \\
02 / 17 / 98 \\
02 / 17 / 98 \\
02 / 17 / 98 \\
02 / 17 / 98 \\
02 / 17 / 98 \\
02 / 17 / 98 \\
02 / 17 / 98 \\
02 / 17 / 98 \\
02 / 17 / 98 \\
02 / 17 / 98 \\
02 / 17 / 98 \\
02 / 17 / 98 \\
02 / 17 / 98 \\
02 / 17 / 98 \\
02 / 17 / 98 \\
02 / 17 / 98 \\
02 / 17 / 98 \\
02 / 17 / 98 \\
02 / 17 / 98 \\
02 / 17 / 98 \\
02 / 17 / 98 \\
02 / 17 / 98 \\
02 / 17 / 98 \\
02 / 17 / 98 \\
02 / 17 / 98 \\
02 / 17 / 98 \\
02 / 17 / 98 \\
02 / 17 / 98\end{array}$ & 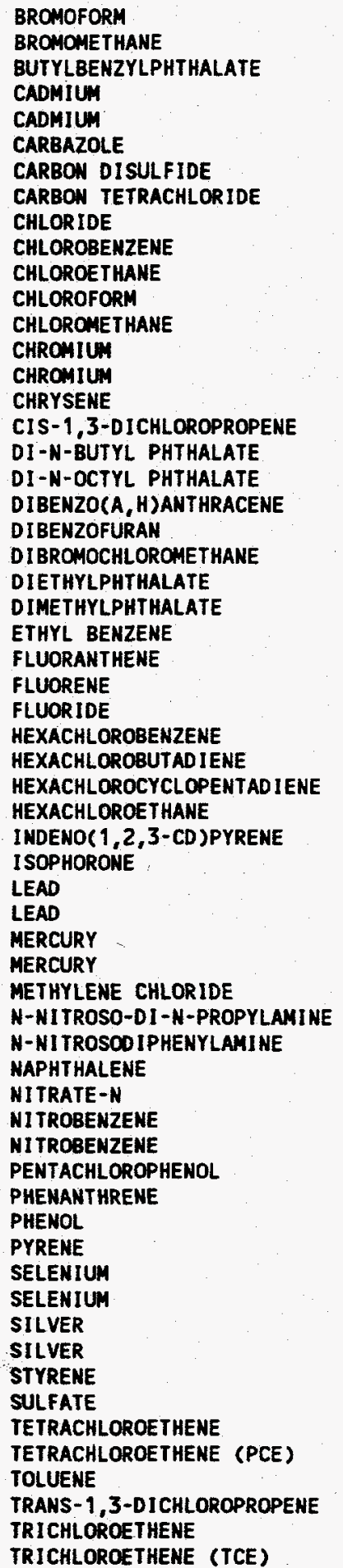 & $\begin{array}{l}\text { ND } \\
\text { ND } \\
\text { ND } \\
\text { ND } \\
\text { ND } \\
\text { ND } \\
\text { ND } \\
\text { ND } \\
28.4 \\
\text { ND } \\
\text { ND } \\
\text { ND } \\
\text { ND } \\
1.6 \\
1.1 \\
\text { ND } \\
\text { ND } \\
\text { ND } \\
\text { ND } \\
\text { ND } \\
\text { ND } \\
\text { ND } \\
\text { ND } \\
\text { ND } \\
\text { ND } \\
\text { ND } \\
\text { ND } \\
\text { ND } \\
\text { ND } \\
\text { ND } \\
\text { ND } \\
\text { ND } \\
\text { ND } \\
\text { ND } \\
\text { ND } \\
\text { ND } \\
1.3 \\
3.7 \\
550 \\
\text { ND } \\
\text { ND } \\
\text { ND } \\
324 \\
\text { ND } \\
\text { ND } \\
\text { ND } \\
\text { ND } \\
\text { ND } \\
\text { ND } \\
3.6 \\
2.7 \\
\text { ND } \\
\text { ND } \\
\text { ND } \\
115 \\
\text { ND } \\
1 \\
\text { ND } \\
\text { ND } \\
850 \\
960\end{array}$ & $\begin{array}{l}100 \\
100 \\
10 \\
0.40 \\
0.40 \\
10 \\
100 \\
100 \\
2.00 \\
100 \\
100 \\
100 \\
100 \\
0.60 \\
0.60 \\
10 \\
100 \\
10 \\
10 \\
10 \\
10 \\
100 \\
10 \\
10 \\
100 \\
10 \\
10 \\
0.50 \\
10 \\
10 \\
10 \\
10 \\
10 \\
10 \\
0.90 \\
0.90 \\
0.10 \\
0.10 \\
100 \\
10 \\
10 \\
10 \\
25.0 \\
10 \\
0.030 \\
50 \\
10 \\
10 \\
10 \\
2.2 \\
2.2 \\
0.80 \\
0.80 \\
100 \\
5.00 \\
100 \\
1 \\
100 \\
100 \\
100 \\
50 \\
0\end{array}$ & 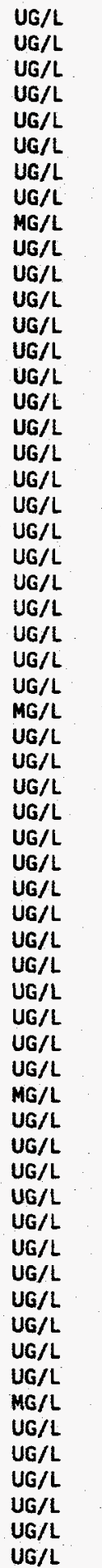 & 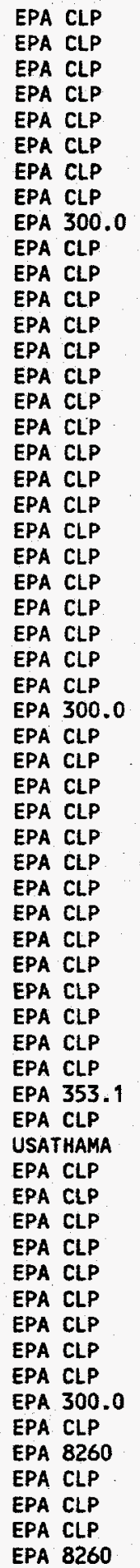 \\
\hline
\end{tabular}




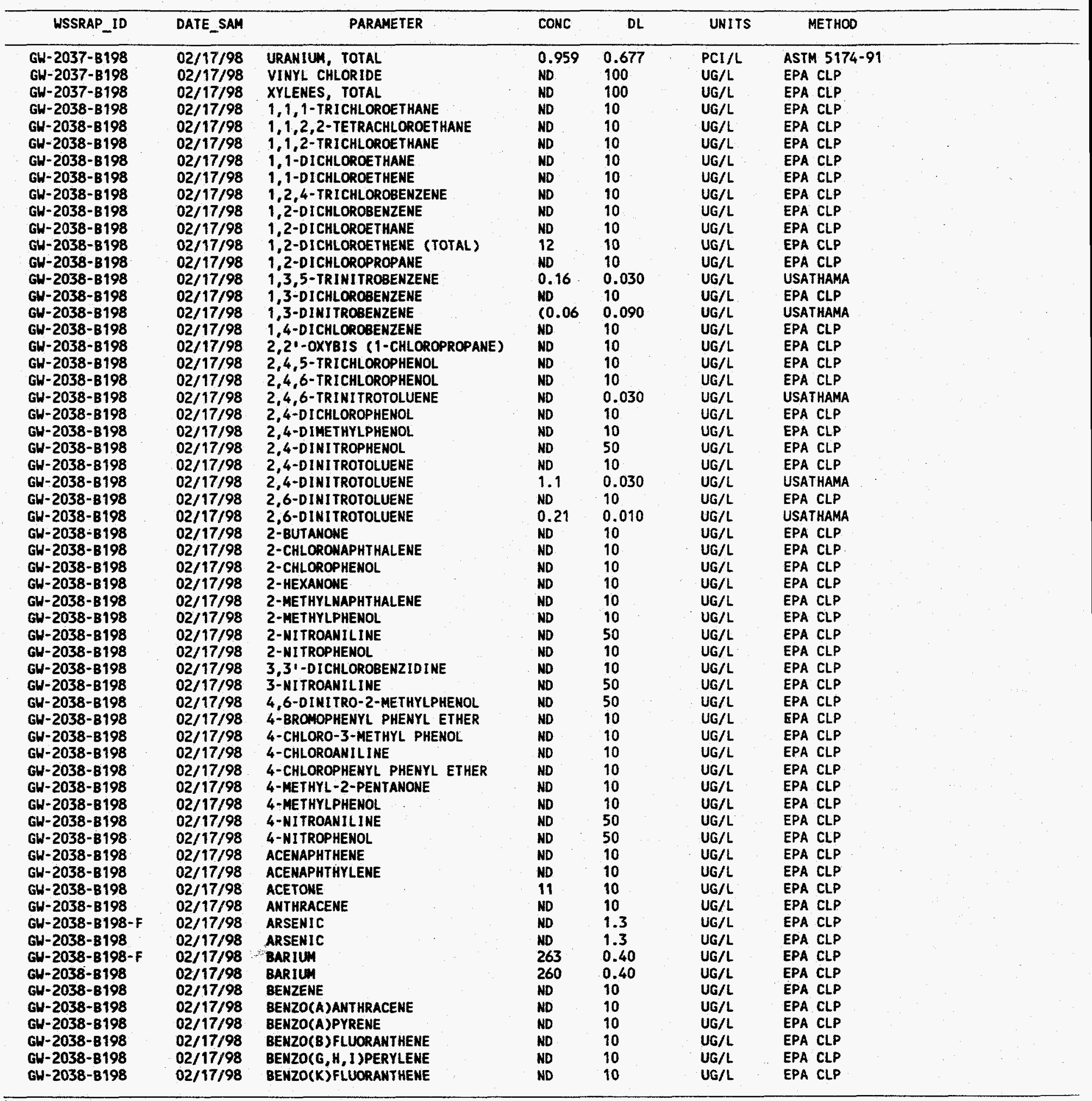




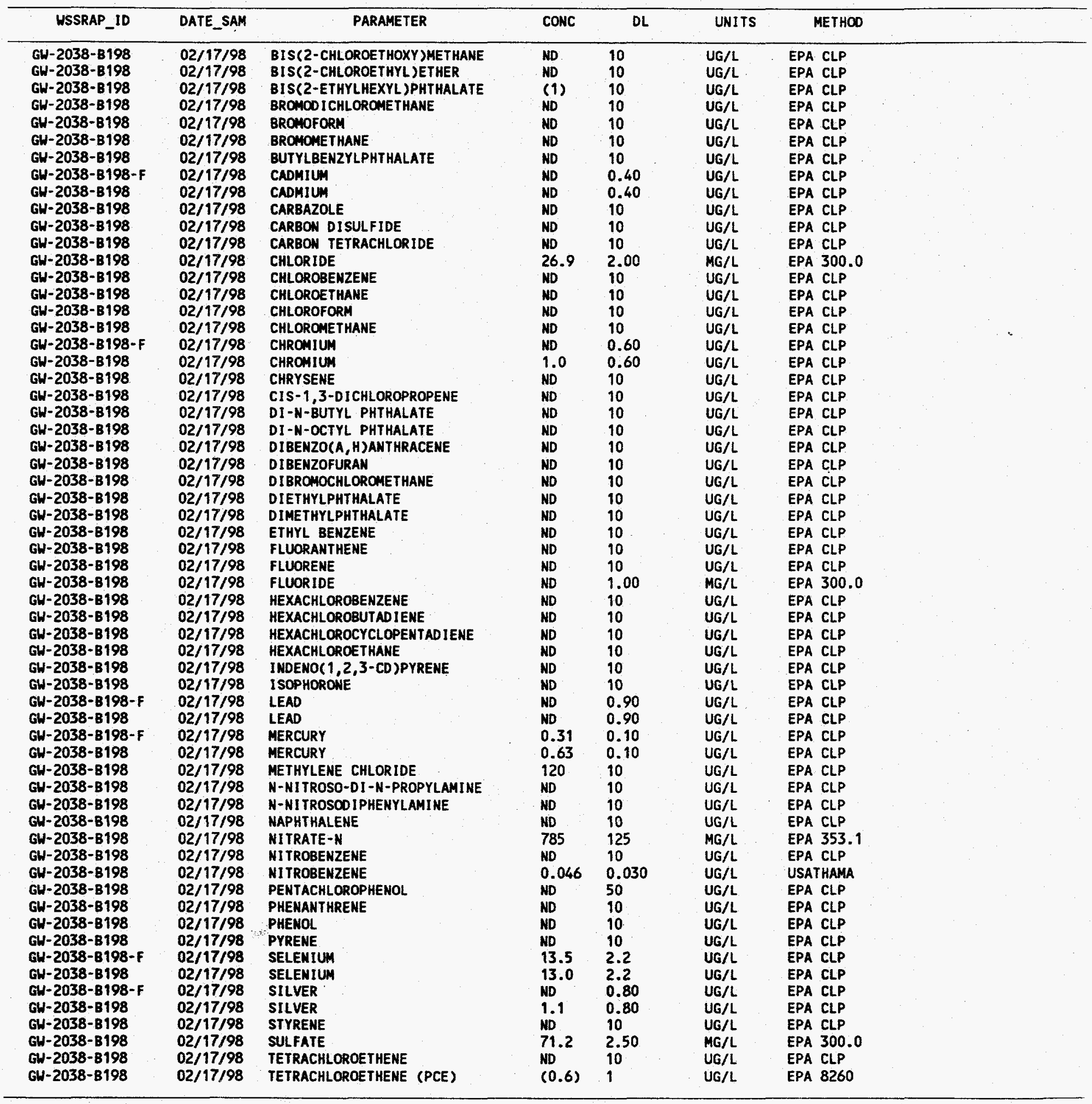




\begin{tabular}{|c|c|c|c|c|c|c|}
\hline WSSRAP_ID & DATE_SAM & PARAMETER & CONC & $D L$ & UNITS & METHOD \\
\hline 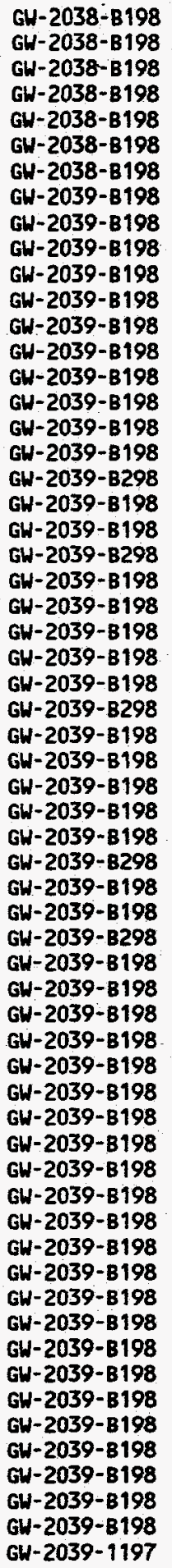 & $\begin{array}{l}02 / 17 / 98 \\
02 / 17 / 98 \\
02 / 17 / 98 \\
02 / 17 / 98 \\
02 / 17 / 98 \\
02 / 17 / 98 \\
02 / 17 / 98 \\
02 / 17 / 98 \\
02 / 17 / 98 \\
02 / 17 / 98 \\
02 / 17 / 98 \\
02 / 17 / 98 \\
02 / 17 / 98 \\
02 / 17 / 98 \\
02 / 17 / 98 \\
02 / 17 / 98 \\
02 / 17 / 98 \\
02 / 17 / 98 \\
04 / 10 / 98 \\
02 / 17 / 98 \\
02 / 17 / 98 \\
04 / 10 / 98 \\
02 / 17 / 98 \\
02 / 17 / 98 \\
02 / 17 / 98 \\
02 / 17 / 98 \\
02 / 17 / 98 \\
04 / 10 / 98 \\
02 / 17 / 98 \\
02 / 17 / 98 \\
02 / 17 / 98 \\
02 / 17 / 98 \\
02 / 17 / 98 \\
04 / 10 / 98 \\
02 / 17 / 98 \\
02 / 17 / 98 \\
04 / 10 / 98 \\
02 / 17 / 98 \\
02 / 17 / 98 \\
02 / 17 / 98 \\
02 / 17 / 98 \\
02 / 17 / 98 \\
02 / 17 / 98 \\
02 / 17 / 98 \\
02 / 17 / 98 \\
02 / 17 / 98 \\
02 / 17 / 98 \\
02 / 17 / 98 \\
02 / 17 / 98 \\
02 / 17 / 98 \\
02 / 17 / 98 \\
02 / 17 / 98 \\
02 / 17 / 98 \\
02 / 17 / 98 \\
02 / 17 / 98 \\
02 / 17 / 98 \\
02 / 17 / 98 \\
02 / 17 / 98 \\
02 / 17 / 98 \\
02 / 17 / 98 \\
11 / 19 / 97\end{array}$ & 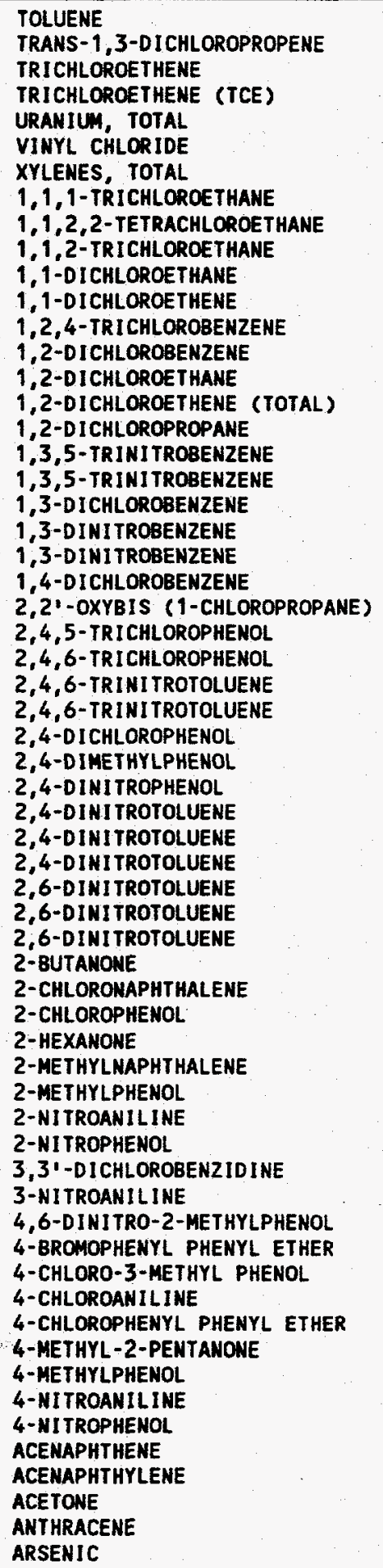 & $\begin{array}{l}\text { ND } \\
\text { ND } \\
380 \\
410 \\
1.48 \\
\text { ND } \\
\text { ND } \\
\text { ND } \\
\text { ND } \\
\text { ND } \\
\text { ND } \\
\text { ND } \\
\text { ND } \\
\text { ND } \\
\text { ND } \\
\text { ND } \\
\text { ND } \\
\text { ND } \\
\text { ND } \\
\text { ND } \\
\text { ND } \\
\text { ND } \\
\text { ND } \\
\text { ND } \\
\text { ND } \\
\text { ND } \\
\text { ND } \\
\text { ND } \\
\text { ND } \\
\text { ND } \\
\text { ND } \\
\text { ND } \\
\text { ND } \\
\text { ND } \\
\text { ND } \\
\text { ND } \\
\text { ND } \\
\text { ND } \\
\text { ND } \\
\text { ND } \\
\text { ND } \\
\text { ND } \\
\text { ND } \\
\text { ND } \\
\text { ND } \\
\text { ND } \\
\text { ND } \\
\text { ND } \\
\text { ND } \\
\text { ND } \\
\text { ND } \\
\text { ND } \\
\text { ND } \\
\text { ND } \\
\text { ND } \\
\text { ND } \\
\text { ND } \\
\text { ND } \\
\text { C9) } \\
\text { ND } \\
\text { ND }\end{array}$ & $\begin{array}{l}10 \\
10 \\
50 \\
50 \\
0.677 \\
10 \\
10 \\
10 \\
10 \\
10 \\
10 \\
10 \\
10 \\
10 \\
10 \\
10 \\
10 \\
0.030 \\
0.030 \\
10 \\
0.090 \\
0.090 \\
10 \\
10 \\
10 \\
10 \\
0.030 \\
0.030 \\
10 \\
10 \\
50 \\
10 \\
0.030 \\
0.030 \\
10 \\
0.010 \\
0.010 \\
10 \\
10 \\
10 \\
10 \\
10 \\
10 \\
50 \\
10 \\
10 \\
50 \\
50 \\
10 \\
10 \\
10 \\
10 \\
10 \\
10 \\
50 \\
50 \\
10 \\
10 \\
10 \\
10 \\
3.00\end{array}$ & $\begin{array}{l}\text { UG } / L \\
U G / L \\
U G / L \\
U G / L \\
P C I / L \\
U G / L \\
U G / L \\
U G / L \\
U G / L \\
U G / L \\
U G / L \\
U G / L \\
U G / L \\
U G / L \\
U G / L \\
U G / L \\
U G / L \\
U G / L \\
U G / L \\
U G / L \\
U G / L \\
U G / L \\
U G / L \\
U G / L \\
U G / L \\
U G / L \\
U G / L \\
U G / L \\
U G / L \\
U G / L \\
U G / L \\
U G / L \\
U G / L \\
U G / L \\
U G / L \\
U G / L \\
U G / L \\
U G / L \\
U G / L \\
U G / L \\
U G / L \\
U G / L \\
U G / L \\
U G / L \\
U G / L \\
U G / L \\
U G / L \\
U G / L \\
U G / L \\
U G / L \\
U G / L \\
U G / L \\
U G / L \\
U G / L \\
U G / L \\
U G / L \\
U G / L \\
U G / L \\
U G / L \\
U G / L \\
U G / L \\
\text { US } / L\end{array}$ & $\begin{array}{l}\text { EPA CLP } \\
\text { EPA CLP } \\
\text { EPA CLP } \\
\text { EPA } 8260 \\
\text { ASTM } 5174-91 \\
\text { EPA CLP } \\
\text { EPA CLP } \\
\text { EPA CLP } \\
\text { EPA CLP } \\
\text { EPA CLP } \\
\text { EPA CLP } \\
\text { EPA CLP } \\
\text { EPA CLP } \\
\text { EPA CLP } \\
\text { EPA CLP } \\
\text { EPA CLP } \\
\text { EPA CLP } \\
\text { USATHAMA } \\
\text { USATHAMA } \\
\text { EPA CLP } \\
\text { USATHAMA } \\
\text { USATHAMA } \\
\text { EPA CLP } \\
\text { EPA CLP } \\
\text { EPA CLP } \\
\text { EPA CLP } \\
\text { USATHAMA } \\
\text { USATHAMA } \\
\text { EPA CLP } \\
\text { EPA CLP } \\
\text { EPA CLP } \\
\text { EPA CLP } \\
\text { USATHAMA } \\
\text { USATHAMA } \\
\text { EPA CLP } \\
\text { USATHAMA } \\
\text { USATHAMA } \\
\text { EPA CLP } \\
\text { EPA CLP } \\
\text { EPA CLP } \\
\text { EPA CLP } \\
\text { EPA CLP } \\
\text { EPA CLP } \\
\text { EPA CLP } \\
\text { EPA CLP } \\
\text { EPA CLP } \\
\text { EPA CLP } \\
\text { EPA CLP } \\
\text { EPA CLP } \\
\text { EPA CLP } \\
\text { EPA CLP } \\
\text { EPA CLP } \\
\text { EPA CLP } \\
\text { EPA CLP } \\
\text { EPA CLP } \\
\text { EPA CLP } \\
\text { EPA CLP } \\
\text { EPA CLP } \\
\text { EPA CLP } \\
\text { EPA CLP } \\
\text { EPA CLP } \\
\end{array}$ \\
\hline
\end{tabular}




\begin{tabular}{|c|c|c|c|c|c|c|}
\hline WSSRAP_ID & DATE_SAM & PARAMETER & CONC & DL & UNITS & METHOD \\
\hline 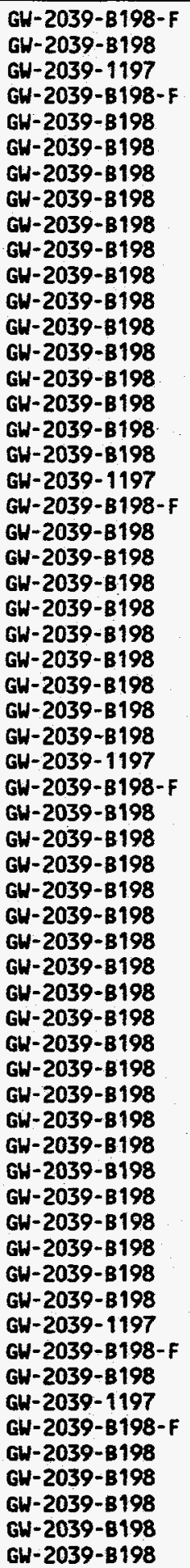 & $\begin{array}{l}02 / 17 / 98 \\
02 / 17 / 98 \\
11 / 19 / 97 \\
02 / 17 / 98 \\
02 / 17 / 98 \\
02 / 17 / 98 \\
02 / 17 / 98 \\
02 / 17 / 98 \\
02 / 17 / 98 \\
02 / 17 / 98 \\
02 / 17 / 98 \\
02 / 17 / 98 \\
02 / 17 / 98 \\
02 / 17 / 98 \\
02 / 17 / 98 \\
02 / 17 / 98 \\
02 / 17 / 98 \\
02 / 17 / 98 \\
11 / 19 / 97 \\
02 / 17 / 98 \\
02 / 17 / 98 \\
02 / 17 / 98 \\
02 / 17 / 98 \\
02 / 17 / 98 \\
02 / 17 / 98 \\
02 / 17 / 98 \\
02 / 17 / 98 \\
02 / 17 / 98 \\
02 / 17 / 98 \\
11 / 19 / 97 \\
02 / 17 / 98 \\
02 / 17 / 98 \\
02 / 17 / 98 \\
02 / 17 / 98 \\
02 / 17 / 98 \\
02 / 17 / 98 \\
02 / 17 / 98 \\
02 / 17 / 98 \\
02 / 17 / 98 \\
02 / 17 / 98 \\
02 / 17 / 98 \\
02 / 17 / 98 \\
02 / 17 / 98 \\
02 / 17 / 98 \\
02 / 17 / 98 \\
02 / 17 / 98 \\
02 / 17 / 98 \\
02 / 17 / 98 \\
02 / 17 / 98 \\
02 / 17 / 98 \\
02 / 17 / 98 \\
11 / 19 / 97 \\
02 / 17 / 98 \\
02 / 17 / 98 \\
11 / 19 / 97 \\
02 / 17 / 98 \\
02 / 17 / 98 \\
02 / 17 / 98 \\
02 / 17 / 98 \\
02 / 17 / 98 \\
02 / 17 / 98\end{array}$ & 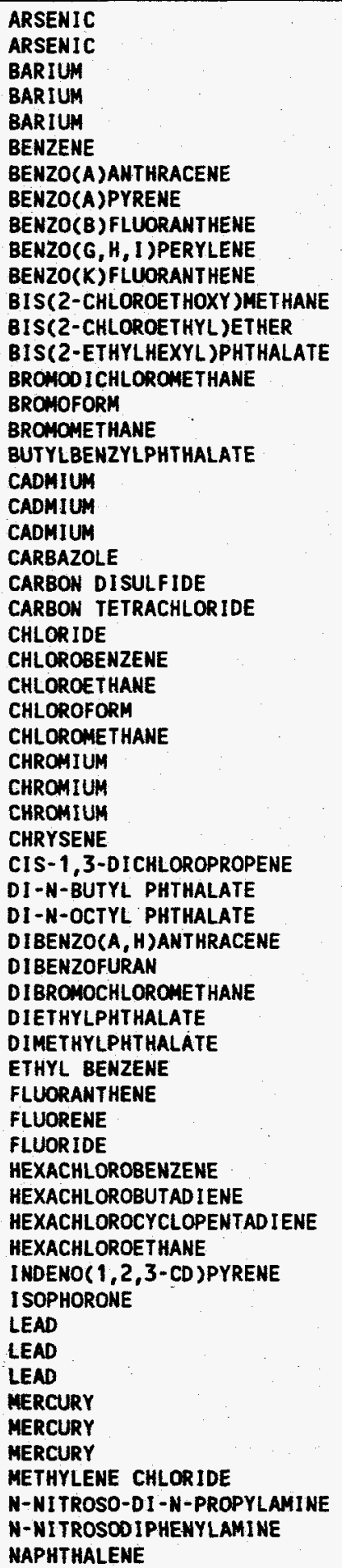 & $\begin{array}{l}\text { ND } \\
\text { ND } \\
341 \\
334 \\
332 \\
\text { ND } \\
\text { ND } \\
\text { ND } \\
\text { ND } \\
\text { ND } \\
\text { ND } \\
\text { ND } \\
\text { ND } \\
\text { ND } \\
\text { ND } \\
\text { ND } \\
\text { ND } \\
\text { ND } \\
\text { ND } \\
\text { ND } \\
\text { ND } \\
\text { ND } \\
\text { ND } \\
\text { ND } \\
46.3 \\
\text { ND } \\
\text { ND } \\
\text { ND } \\
\text { ND } \\
5.00 \\
3.1 \\
3.1 \\
\text { ND } \\
\text { ND } \\
\text { (3) } \\
\text { ND } \\
\text { ND } \\
\text { ND } \\
\text { ND } \\
\text { ND } \\
\text { ND } \\
\text { ND } \\
\text { ND } \\
\text { ND } \\
0.31 \\
\text { ND } \\
\text { ND } \\
\text { ND } \\
\text { ND } \\
\text { ND } \\
\text { ND } \\
\text { ND } \\
\text { ND } \\
\text { ND } \\
\text { ND } \\
\text { ND } \\
1200 \\
\text { ND } \\
\text { ND } \\
\text { ND } \\
\text { ND } \\
\text { ND } \\
\text { No }\end{array}$ & $\begin{array}{l}1.3 \\
1.3 \\
1.00 \\
0.40 \\
0.40 \\
10 \\
10 \\
10 \\
10 \\
10 \\
10 \\
10 \\
10 \\
10 \\
10 \\
10 \\
10 \\
10 \\
10 \\
1.00 \\
0.40 \\
0.40 \\
10 \\
10 \\
10 \\
4.00 \\
10 \\
10 \\
10 \\
10 \\
10 \\
20 \\
2.00 \\
0.60 \\
0.60 \\
10\end{array}$ & $\begin{array}{l}U G / L \\
U G / L \\
U G / L \\
U G / L \\
U G / L \\
U G / L \\
U G / L \\
U G / L \\
U G / L \\
U G / L \\
U G / L \\
U G / L \\
U G / L \\
U G / L \\
U G / L \\
U G / L \\
U G / L \\
U G / L \\
U G / L \\
U G / L \\
U G / L \\
U G / L \\
U G / L \\
U G / L \\
U G / L \\
U G / L \\
U G / L \\
U G / L \\
U G / L \\
U G / L \\
U G / L \\
U G / L \\
U G / L \\
U G / L \\
U G / L \\
U G / L \\
U G / L \\
U G / L \\
U G / L \\
U G / L \\
U G / L \\
U G / L \\
U G / L \\
U G / L \\
U G / L \\
U G / L \\
U G / L \\
U G / L \\
U G / L \\
U G / L \\
U G / L \\
U G / L \\
U G / L \\
U G / L \\
U G / L \\
U G / L \\
U G / L \\
U G / L \\
U G / L \\
U G / L \\
U G / L \\
U\end{array}$ & $\begin{array}{l}\text { EPA CLP } \\
\text { EPA CLP } \\
\text { EPA CLP } \\
\text { EPA CLP } \\
\text { EPA CLP } \\
\text { EPA CLP } \\
\text { EPA CLP } \\
\text { EPA CLP } \\
\text { EPA CLP } \\
\text { EPA CLP } \\
\text { EPA CLP } \\
\text { EPA CLP } \\
\text { EPA CLP } \\
\text { EPA CLP } \\
\text { EPA CLP } \\
\text { EPA CLP } \\
\text { EPA CLP } \\
\text { EPA CLP } \\
\text { EPA CLP } \\
\text { EPA CLP } \\
\text { EPA CLP } \\
\text { EPA CLP } \\
\text { EPA CLP } \\
\text { EPA CLP } \\
\text { EPA } 300.0 \\
\text { EPA CLP } \\
\text { EPA CLP } \\
\text { EPA CLP } \\
\text { EPA CLP } \\
\text { EPA CLP } \\
\text { EPA CLP } \\
\text { EPA CLP } \\
\text { EPA CLP } \\
\text { EPA CLP } \\
\text { EPA CLP } \\
\text { EPA CLP } \\
\text { EPA CLP } \\
\text { EPA CLP } \\
\text { EPA CLP } \\
\text { EPA CLP } \\
\text { EPA CLP } \\
\text { EPA CLP } \\
\text { EPA CLP } \\
\text { EPA CLP } \\
\text { EPA } 300.0 \\
\text { EPA CLP } \\
\text { EPA CLP } \\
\text { EPA CLP } \\
\text { EPA CLP } \\
\text { EPA CLP } \\
\text { EPA CLP } \\
\text { EPA CLP } \\
\text { EPA CLP } \\
\text { EPA CLP } \\
\text { EPA CLP } \\
\text { EPA CLP } \\
\text { EPA CLP } \\
\text { EPA CLP } \\
\text { EPA CLP } \\
\text { EPA CLP } \\
\text { EPA CLPP }\end{array}$ \\
\hline
\end{tabular}




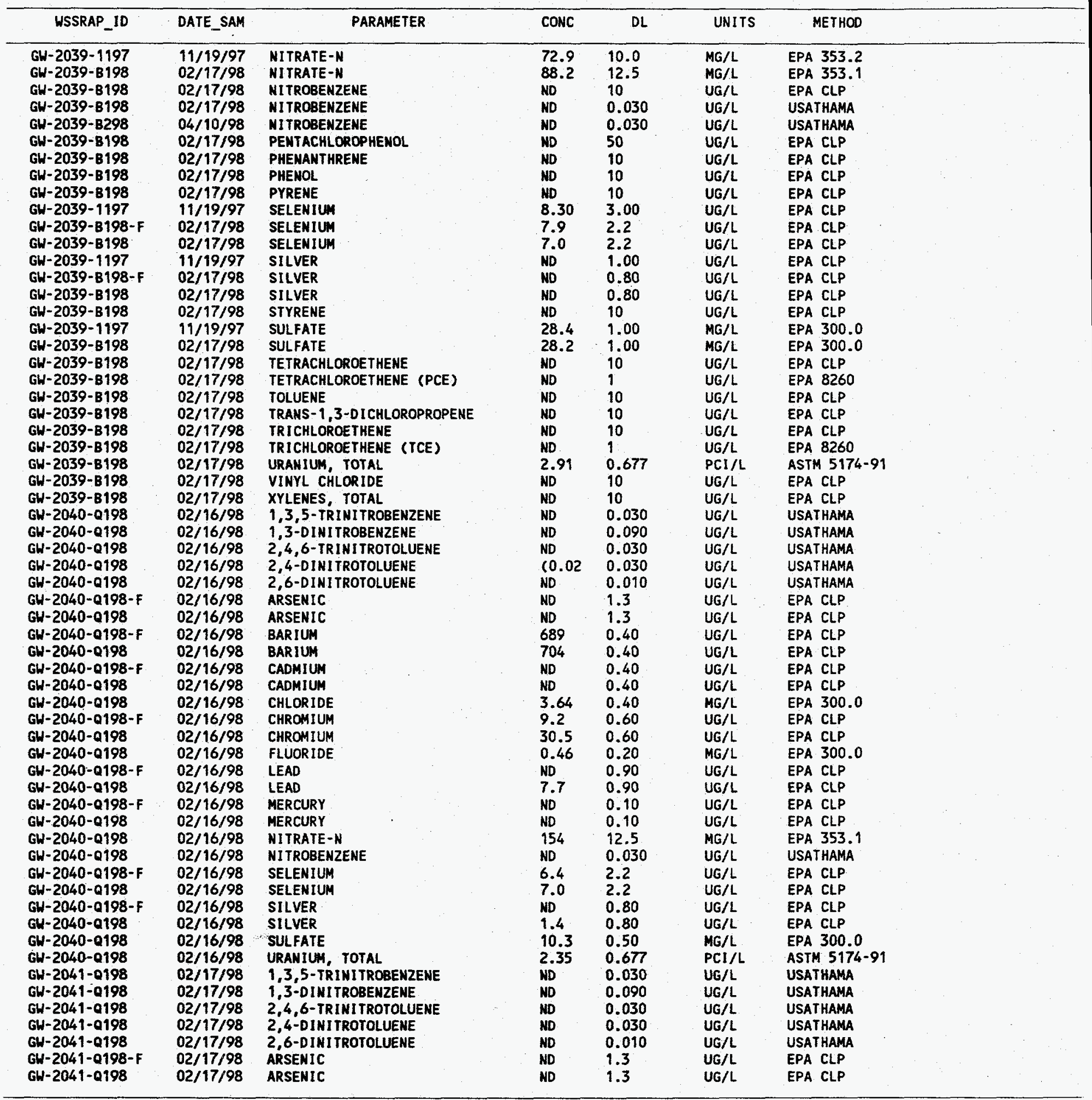




\begin{tabular}{|c|c|c|c|c|c|c|}
\hline WSSRAP_ID & DATE_SAM & PARAMETER & CONC & $D L$ & UNITS & METHOD \\
\hline 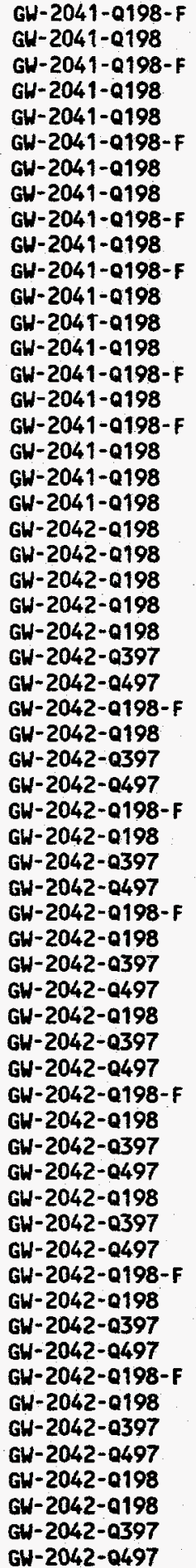 & $\begin{array}{l}02 / 17 / 98 \\
02 / 17 / 98 \\
02 / 17 / 98 \\
02 / 17 / 98 \\
02 / 17 / 98 \\
02 / 17 / 98 \\
02 / 17 / 98 \\
02 / 17 / 98 \\
02 / 17 / 98 \\
02 / 17 / 98 \\
02 / 17 / 98 \\
02 / 17 / 98 \\
02 / 17 / 98 \\
02 / 17 / 98 \\
02 / 17 / 98 \\
02 / 17 / 98 \\
02 / 17 / 98 \\
02 / 17 / 98 \\
02 / 17 / 98 \\
02 / 17 / 98 \\
02 / 16 / 98 \\
02 / 16 / 98 \\
02 / 16 / 98 \\
02 / 16 / 98 \\
02 / 16 / 98 \\
09 / 22 / 97 \\
11 / 19 / 97 \\
02 / 16 / 98 \\
02 / 16 / 98 \\
09 / 22 / 97 \\
11 / 19 / 97 \\
02 / 16 / 98 \\
02 / 16 / 98 \\
09 / 22 / 97 \\
11 / 19 / 97 \\
02 / 16 / 98 \\
02 / 16 / 98 \\
09 / 22 / 97 \\
11 / 19 / 97 \\
02 / 16 / 98 \\
09 / 22 / 97 \\
11 / 19 / 97 \\
02 / 16 / 98 \\
02 / 16 / 98 \\
09 / 22 / 97 \\
11 / 19 / 97 \\
02 / 16 / 98 \\
09 / 22 / 97 \\
11 / 19 / 97 \\
02 / 16 / 98 \\
02 / 16 / 98 \\
09 / 22 / 97 \\
11 / 19 / 97 \\
02 / 16 / 98 \\
02 / 16 / 98 \\
09 / 22 / 97 \\
11 / 19 / 97 \\
02 / 16 / 98 \\
02 / 16 / 98 \\
09 / 22 / 97 \\
11 / 19 / 97\end{array}$ & 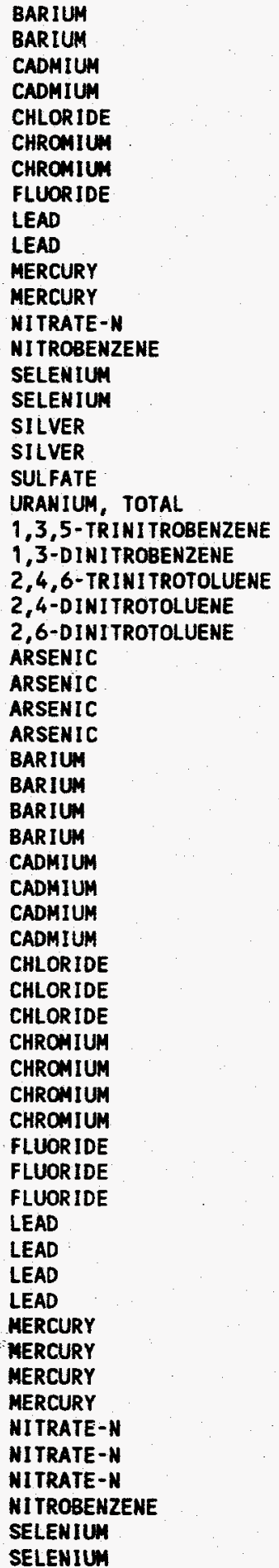 & 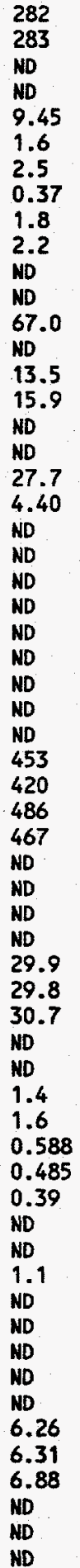 & $\begin{array}{l}0.40 \\
0.40 \\
0.40 \\
0.40 \\
0.40 \\
0.60 \\
0.60 \\
0.20 \\
0.90 \\
0.90 \\
0.10 \\
0.10 \\
5.00 \\
0.030 \\
2.2 \\
2.2 \\
0.80 \\
0.80 \\
1.00 \\
0.677 \\
0.030 \\
0.090 \\
0.030 \\
0.030 \\
0.010 \\
2.00 \\
3.00 \\
1.3 \\
1.3 \\
5.00 \\
1.00 \\
0.40 \\
0.40 \\
5.00 \\
1.00 \\
0.40 \\
0.40 \\
0.250 \\
0.250 \\
2.00 \\
10.0 \\
2.00 \\
0.60 \\
0.60 \\
0.200 \\
0.200 \\
0.20 \\
1.50 \\
2.00 \\
0.90 \\
0.90 \\
0.100 \\
0.100 \\
0.10 \\
0.10 \\
1.00 \\
0.400 \\
1.00 \\
0.030 \\
2.50 \\
3.00\end{array}$ & $\begin{array}{l}U G / L \\
U G / L \\
U G / L \\
U G / L \\
M G / L \\
U G / L \\
U G / L \\
M G / L \\
U G / L \\
U G / L \\
U G / L \\
U G / L \\
M G / L \\
U G / L \\
U G / L \\
U G / L \\
U G / L \\
U G / L \\
M G / L \\
P C I / L \\
U G / L \\
U G / L \\
U G / L \\
U G / L \\
U G / L \\
U G / L \\
U G / L \\
U G / L \\
U G / L \\
U G / L \\
U G / L \\
U G / L \\
U G / L \\
U G / L \\
U G / L \\
U G / L \\
U G / L \\
M G / L \\
M G / L \\
M G / L \\
U G / L \\
U G / L \\
U G / L \\
U G / L \\
M G / L \\
M G / L \\
M G / L \\
U G / L \\
U G / L \\
U G / L \\
U G / L \\
U G / L \\
U G / L \\
U G / L \\
U G / L \\
M G / L \\
M G / L \\
M G / L \\
U G / L \\
U G / L \\
U G / L\end{array}$ & $\begin{array}{l}\text { EPA CLP } \\
\text { EPA CLP } \\
\text { EPA CLP } \\
\text { EPA CLP } \\
\text { EPA } 300.0 \\
\text { EPA CLP } \\
\text { EPA CLP } \\
\text { EPA } 300.0 \\
\text { EPA CLP } \\
\text { EPA CLP } \\
\text { EPA CLP } \\
\text { EPA CLP } \\
\text { EPA } 353.1 \\
\text { USATHAMA } \\
\text { EPA CLP } \\
\text { EPA CLP } \\
\text { EPA CLP } \\
\text { EPA CLP } \\
\text { EPA } 300.0 \\
\text { ASTM } 5174-91 \\
\text { USATHAMA } \\
\text { USATHAMA } \\
\text { USATHAMA } \\
\text { USATHAMA } \\
\text { USATHAMA } \\
\text { EPA CLP } \\
\text { EPA CLP } \\
\text { EPA CLP } \\
\text { EPA CLP } \\
\text { EPA CLP } \\
\text { EPA CLP } \\
\text { EPA CLP } \\
\text { EPA CLP } \\
\text { EPA CLP } \\
\text { EPA CLP } \\
\text { EPA CLP } \\
\text { EPA CLP } \\
\text { EPA } 300.0 \\
\text { EPA } 300.0 \\
\text { EPA } 300.0 \\
\text { EPA CLP } \\
\text { EPA CLP } \\
\text { EPA CLP } \\
\text { EPA CLP } \\
\text { EPA } 300.0 \\
\text { EPA } 300.0 \\
\text { EPA } 300.0 \\
\text { EPA CLP } \\
\text { EPA CLP } \\
\text { EPA CLP } \\
\text { EPA CLP } \\
\text { EPA CLP } \\
\text { EPA CLP } \\
\text { EPA CLP } \\
\text { EPA CLP } \\
\text { EPA } 353.2 \\
\text { EPA } 353.2 \\
\text { EPA } 353.1 \\
\text { USATHAMA } \\
\text { EPA CLP } \\
\text { EPA CLP }\end{array}$ \\
\hline
\end{tabular}




\begin{tabular}{|c|c|c|c|c|c|c|}
\hline WSSRAP_ID & DATE_SAM & PARAMETER & CONC & $\mathrm{DL}$ & UNITS & METHOD \\
\hline $\begin{array}{l}\text { GW-2042-Q198-F } \\
G W-2042-0198 \\
G W-2042-0397 \\
G W-2042-0497 \\
G W-2042-0198-F \\
G W-2042-Q 198 \\
G W-2042-0397 \\
G W-2042-0497 \\
G W-2042-0198 \\
G W-2042-0397 \\
G W-2042-0497 \\
G W-2042-0198 \\
G W-2043-0198 \\
G W-2043-0198 \\
G W-2043-0198 \\
G W-2043-0198 \\
G W-2043-0198 \\
G W-2043-0397 \\
G W-2043-0497 \\
G W-2043-0198-F \\
G W-2043-0198 \\
G W-2043-0397 \\
G W-2043-0497 \\
G W-2043-0198-F \\
G W-2043-0198 \\
G W-2043-0397 \\
G H-2043-0497 \\
G W-2043-0198-F \\
G W-2043-0198 \\
G W-2043-0397 \\
G W-2043-0497 \\
G W-2043-0198 \\
G W-2043-0397 \\
G W-2043-0497 \\
G W-2043-0198-F \\
G W-2043-0198 \\
G W-2043-0397 \\
G W-2043-0497 \\
G W-2043-0198 \\
G W-2043-0397 \\
G W-2043-0497 \\
G W-2043-0198-F \\
G W-2043-0198 \\
G W-2043-0397 \\
G W-2043-0497 \\
G W-2043-0198-F \\
G W-2043-0198 \\
G W-2043-0397 \\
G W-2043-0497 \\
G W-2043-0198 \\
G W-2043-0198 \\
G W-2043-0397 \\
G W-2043-0497 \\
G W-2043-0198-F \\
G W-2043-Q 198 \\
G W-2043-0397 \\
G W-2043-0497 \\
G W-2043-0198-F \\
G W-2043-0198 \\
G W-2043-0397 \\
G W-2043-0497\end{array}$ & $\begin{array}{l}02 / 16 / 98 \\
02 / 16 / 98 \\
09 / 22 / 97 \\
11 / 19 / 97 \\
02 / 16 / 98 \\
02 / 16 / 98 \\
09 / 22 / 97 \\
11 / 19 / 97 \\
02 / 16 / 98 \\
09 / 22 / 97 \\
11 / 19 / 97 \\
02 / 16 / 98 \\
02 / 16 / 98 \\
02 / 16 / 98 \\
02 / 16 / 98 \\
02 / 16 / 98 \\
02 / 16 / 98 \\
09 / 22 / 97 \\
11 / 19 / 97 \\
02 / 16 / 98 \\
02 / 16 / 98 \\
09 / 22 / 97 \\
11 / 19 / 97 \\
02 / 16 / 98 \\
02 / 16 / 98 \\
09 / 22 / 97 \\
11 / 19 / 97 \\
02 / 16 / 98 \\
02 / 16 / 98 \\
09 / 22 / 97 \\
11 / 19 / 97 \\
02 / 16 / 98 \\
09 / 22 / 97 \\
11 / 19 / 97 \\
02 / 16 / 98 \\
02 / 16 / 98 \\
09 / 22 / 97 \\
11 / 19 / 97 \\
02 / 16 / 98 \\
09 / 22 / 97 \\
11 / 19 / 97 \\
02 / 16 / 98 \\
02 / 16 / 98 \\
09 / 22 / 97 \\
11 / 19 / 97 \\
02 / 16 / 98 \\
02 / 16 / 98 \\
09 / 22 / 97 \\
11 / 19 / 97 \\
02 / 16 / 98 \\
02 / 16 / 98 \\
09 / 22 / 97 \\
11 / 19 / 97 \\
02 / 16 / 98 \\
02 / 16 / 98 \\
09 / 22 / 97 \\
11 / 19 / 97 \\
02 / 16 / 98 \\
02 / 16 / 98 \\
09 / 22 / 97 \\
11 / 119 / 97\end{array}$ & 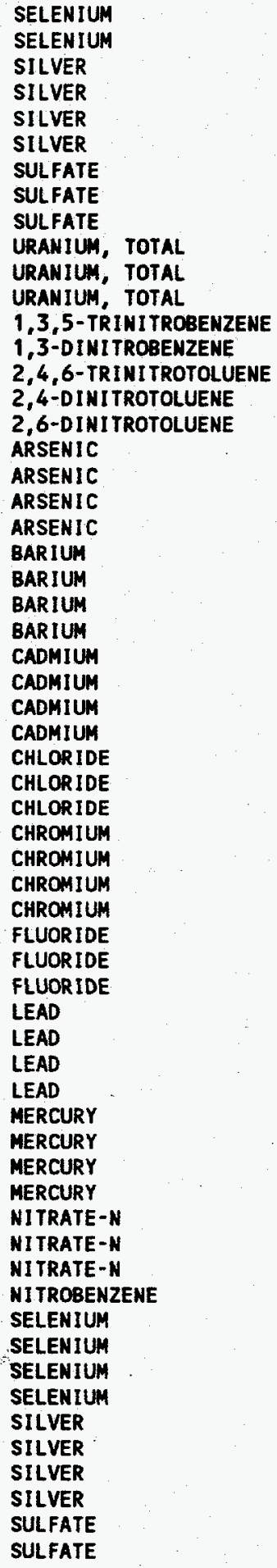 & $\begin{array}{l}3.4 \\
\text { ND } \\
\text { ND } \\
\text { ND } \\
\text { ND } \\
31.9 \\
32.0 \\
34.0 \\
2.71 \\
2.78 \\
2.64 \\
\text { ND.01 } \\
\text { ND } \\
0.077 \\
\text { ND } \\
\text { ND } \\
\text { ND } \\
\text { ND } \\
\text { ND } \\
336 \\
340 \\
353 \\
362 \\
\text { ND } \\
\text { ND } \\
\text { ND } \\
\text { ND } \\
4.66 \\
6.83 \\
5.02 \\
\text { ND } \\
3.80 \\
2.3 \\
3.6 \\
0.482 \\
\text { ND } \\
0.32 \\
\text { ND } \\
\text { ND } \\
\text { ND } \\
3.2 \\
\text { ND } \\
\text { ND } \\
\text { ND } \\
\text { ND } \\
6.61 \\
494 \\
6.83 \\
\text { ND } \\
2.90 \\
4.00 \\
3.2 \\
2.5 \\
\text { ND } \\
\text { ND } \\
\text { ND } \\
\text { ND } \\
14.6 \\
10.0\end{array}$ & 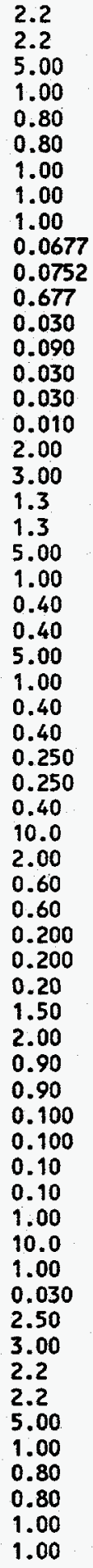 & $\begin{array}{l}U G / L \\
U G / L \\
U G / L \\
U G / L \\
U G / L \\
U G / L \\
M G / L \\
M G / L \\
M G / L \\
P C I / L \\
P C I / L \\
P C I / L \\
U G / L \\
U G / L \\
U G / L \\
U G / L \\
U G / L \\
U G / L \\
U G / L \\
U G / L \\
U G / L \\
U G / L \\
U G / L \\
U G / L \\
U G / L \\
U G / L \\
U G / L \\
U G / L \\
U G / L \\
M G / L \\
M G / L \\
M G / L \\
U G / L \\
U G / L \\
U G / L \\
U G / L \\
M G / L \\
M G / L \\
M G / L \\
U G / L \\
U G / L \\
U G / L \\
U G / L \\
U G / L \\
U G / L \\
U G / L \\
U G / L \\
M G / L \\
M G / L \\
U G / L \\
U G / L \\
U G / L \\
U G / L \\
U G / L \\
U G / L \\
U G / L \\
U G / L \\
U G / L \\
U G / L \\
M G / L \\
M G / L \\
U\end{array}$ & $\begin{array}{l}\text { EPA CLP } \\
\text { EPA CLP } \\
\text { EPA CLP } \\
\text { EPA CLP } \\
\text { EPA CLP } \\
\text { EPA CLP } \\
\text { EPA } 300.0 \\
\text { EPA } 300.0 \\
\text { EPA } 300.0 \\
\text { EPA } 6020 \\
\text { EPA } 6020 \\
\text { ASTM } 5174-91 \\
\text { USATHAMA } \\
\text { USATHAMA } \\
\text { USATHAMA } \\
\text { USATHAMA } \\
\text { USATHAMA } \\
\text { EPA CLP } \\
\text { EPA CLP } \\
\text { EPA CLP } \\
\text { EPA CLP } \\
\text { EPA CLP } \\
\text { EPA CLP } \\
\text { EPA CLP } \\
\text { EPA CLP } \\
\text { EPA CLP } \\
\text { EPA CLP } \\
\text { EPA CLP } \\
\text { EPA CLP } \\
\text { EPA } 300.0 \\
\text { EPA } 300.0 \\
\text { EPA } 300.0 \\
\text { EPA CLP } \\
\text { EPA CLP } \\
\text { EPA CLP } \\
\text { EPA CLP } \\
\text { EPA } 300.0 \\
\text { EPA } 300.0 \\
\text { EPA } 300.0 \\
\text { EPA CLP } \\
\text { EPA CLP } \\
\text { EPA CLP } \\
\text { EPA CLP } \\
\text { EPA CLP } \\
\text { EPA CLP } \\
\text { EPA CLP } \\
\text { EPA CLP } \\
\text { EPA } 353.2 \\
\text { EPA } 353.2 \\
\text { EPA } 353.1 \\
\text { USATHAMA } \\
\text { EPA CLP } \\
\text { EPA CLP } \\
\text { EPA CLP } \\
\text { EPA CLP } \\
\text { EPA CLP } \\
\text { EPA CLP } \\
\text { EPA CLP } \\
\text { EPA CLP } \\
\text { EPA } 300.0 \\
\text { EPA } 300.0\end{array}$ \\
\hline
\end{tabular}




\begin{tabular}{|c|c|c|c|c|c|c|}
\hline WSSRAP_ID & DATE_SAM & PARAMETER & CONC & DL & UNITS & METHOD \\
\hline $\begin{array}{l}G W-2043-0198 \\
G W-2043-0397 \\
G W-2043-0497 \\
G W-2043-0198 \\
G W-2045-Q 198-C 1 \\
G W-2045-0198-D \\
G W-2045-0198-C 1 \\
G W-2045-0198-D \\
G W-2045-Q 198-C 1 \\
G W-2045-0198-D \\
G W-2045-0198-C 1 \\
G W-2045-0198-D \\
G W-2045-0198-C 1 \\
G W-2045-0198-D \\
G W-2045-0198-C 1 \\
G W-2045-0198-D \\
G W-2045-0198-C 1 \\
G W-2045-Q 198-D \\
G W-2045-0198-C 1 \\
G W-2045-0198-D \\
G W-2045-0198-A \\
G W-2045-0198-B \\
G W-2045-0198-D \\
G W-2045-0298-B \\
G W-2045-0298-C \\
G W-2045-0198-A \\
G W-2045-0198-B \\
G W-2045-0198-D \\
G W-2045-0298-B \\
G W-2045-0298-C \\
G W-2045-0198-A \\
G W-2045-0198-B \\
G W-2045-Q 198-D \\
G W-2045-0298-B \\
G W-2045-0298-C \\
G W-2045-0198-A \\
G W-2045-0198-B \\
G W-2045-0198-D \\
G W-2045-0298-B \\
G W-2045-0298-C \\
G W-2045-0198-A \\
G W-2045-0198-B \\
G W-2045-0198-D \\
G W-2045-0298-B \\
G W-2045-0298-C \\
G W-2045-0198-C 1 \\
G W-2045-0198-D \\
G W-2045-0198-C 1 \\
G W-2045-0198-D \\
G W-2045-0198-C 1 \\
G W-2045-0198-D \\
G W-2045-0198-C 1 \\
G W-2045-0198-D \\
G W-2045-0198-C 1 \\
G W-2045-0198-D \\
G W-2045-0198-C 1 \\
G W-2045-0198-D \\
G W-2045-0198-C \\
G W-2045-0198-D 1 \\
G W-2045-0198-C 1 \\
G W-2045-0198-D\end{array}$ & $\begin{array}{l}02 / 16 / 98 \\
09 / 22 / 97 \\
11 / 19 / 97 \\
02 / 16 / 98 \\
01 / 28 / 98 \\
01 / 29 / 98 \\
01 / 28 / 98 \\
01 / 29 / 98 \\
01 / 28 / 98 \\
01 / 29 / 98 \\
01 / 28 / 98 \\
01 / 29 / 98 \\
01 / 28 / 98 \\
01 / 29 / 98 \\
01 / 28 / 98 \\
01 / 29 / 98 \\
01 / 28 / 98 \\
01 / 29 / 98 \\
01 / 28 / 98 \\
01 / 29 / 98 \\
01 / 21 / 98 \\
01 / 23 / 98 \\
01 / 29 / 98 \\
04 / 08 / 98 \\
04 / 10 / 98 \\
01 / 21 / 98 \\
01 / 23 / 98 \\
01 / 29 / 98 \\
04 / 08 / 98 \\
04 / 10 / 98 \\
01 / 21 / 98 \\
01 / 23 / 98 \\
01 / 29 / 98 \\
04 / 08 / 98 \\
04 / 10 / 98 \\
01 / 21 / 98 \\
01 / 23 / 98 \\
01 / 29 / 98 \\
04 / 08 / 98 \\
04 / 10 / 98 \\
01 / 21 / 98 \\
01 / 23 / 98 \\
01 / 29 / 98 \\
04 / 08 / 98 \\
04 / 10 / 98 \\
01 / 28 / 98 \\
01 / 29 / 98 \\
01 / 28 / 98 \\
01 / 29 / 98 \\
01 / 28 / 98 \\
01 / 29 / 98 \\
01 / 28 / 98 \\
01 / 29 / 98 \\
01 / 28 / 98 \\
01 / 29 / 98 \\
01 / 28 / 98 \\
01 / 29 / 98 \\
01 / 27 / 98 \\
02 / 02 / 98 \\
01 / 28 / 98 \\
01 / 29 / 98\end{array}$ & 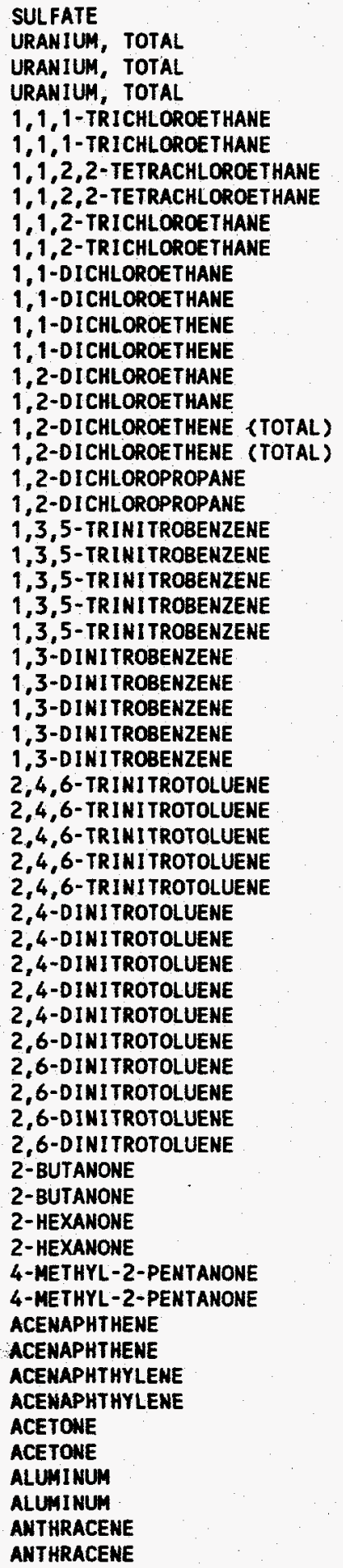 & $\begin{array}{l}14.8 \\
1.81 \\
1.96 \\
1.69 \\
\text { ND } \\
\text { ND } \\
\text { ND } \\
\text { ND } \\
\text { ND } \\
\text { ND } \\
\text { ND } \\
\text { ND } \\
\text { ND } \\
\text { ND } \\
\text { ND } \\
\text { ND } \\
\text { ND } \\
\text { ND } \\
\text { ND } \\
\text { ND } \\
\text { C.02 } \\
C 0.02 \\
0.034 \\
0.031 \\
0.038 \\
0.18 \\
0.13 \\
0.16 \\
0.15 \\
0.15 \\
\text { ND } \\
\text { ND } \\
\text { ND } \\
\text { ND } \\
\text { ND } \\
0.13 \\
0.12 \\
0.14 \\
0.13 \\
0.13 \\
0.65 \\
0.74 \\
0.74 \\
0.68 \\
0.73 \\
\text { ND } \\
\text { ND } \\
\text { ND } \\
\text { ND } \\
\text { ND } \\
\text { ND } \\
\text { ND } \\
\text { ND } \\
\text { ND } \\
\text { ND } \\
(5) \\
\text { C15) } \\
51.8 \\
37.6 \\
\text { ND } \\
\text { ND }\end{array}$ & $\begin{array}{l}0.50 \\
0.0677 \\
0.0752 \\
0.077 \\
10 \\
20 \\
10 \\
10 \\
20 \\
10 \\
20 \\
10 \\
10 \\
20 \\
10 \\
20 \\
10 \\
10 \\
20 \\
10 \\
20 \\
10 \\
10 \\
20 \\
0.030 \\
0.030 \\
0\end{array}$ & $\begin{array}{l}M G / L \\
P C I / L \\
P C I / L \\
P C I / L \\
U G / L \\
U G / L \\
U G / L \\
U G / L \\
U G / L \\
U G / L \\
U G / L \\
U G / L \\
U G / L \\
U G / L \\
U G / L \\
U G / L \\
U G / L \\
U G / L \\
U G / L \\
U G / L \\
U G / L \\
U G / L \\
U G / L \\
U G / L \\
U G / L \\
U G / L \\
U G / L \\
U G / L \\
U G / L \\
U G / L \\
U G / L \\
U G / L \\
U G / L \\
U G / L \\
U G / L \\
U G / L \\
U G / L \\
U G / L \\
U G / L \\
U G / L \\
U G / L \\
U G / L \\
U G / L \\
U G / L \\
U G / L \\
U G / L \\
U G / L \\
U G / L \\
U G / L \\
U G / L \\
U G / L \\
U G / L \\
U G / L \\
U G / L \\
U G / L \\
U G / L \\
U G / L \\
U G / L \\
U G / L \\
U G / L \\
U G / L \\
U\end{array}$ & $\begin{array}{l}\text { EPA } 300.0 \\
\text { EPA } 6020 \\
\text { EPA } 6020 \\
\text { ASTM } 5174-91 \\
\text { EPA CLP } \\
\text { EPA CLP } \\
\text { EPA CLP } \\
\text { EPA CLP } \\
\text { EPA CLP } \\
\text { EPA CLP } \\
\text { EPA CLP } \\
\text { EPA CLP } \\
\text { EPA CLP } \\
\text { EPA CLP } \\
\text { EPA CLP } \\
\text { EPA CLP } \\
\text { EPA CLP } \\
\text { EPA CLP } \\
\text { EPA CLP } \\
\text { EPA CLP } \\
\text { USATHAMA } \\
\text { USATHAMA } \\
\text { USATHAMA } \\
\text { USATHAMA } \\
\text { USATHAMA } \\
\text { USATHAMA } \\
\text { USATHAMA } \\
\text { USATHAMA } \\
\text { USATHAMA } \\
\text { USATHAMA } \\
\text { USATHAMA } \\
\text { USATHAMA } \\
\text { USATHAMA } \\
\text { USATHAMA } \\
\text { USATHAMA } \\
\text { USATHAMA } \\
\text { USATHAMA } \\
\text { USATHAMA } \\
\text { USATHAMA } \\
\text { USATHAMA } \\
\text { USATHAMA } \\
\text { USATHAMA } \\
\text { USATHAMA } \\
\text { USATHAMA } \\
\text { USATHAMA } \\
\text { EPA CLP } \\
\text { EPA CLP } \\
\text { EPA CLP } \\
\text { EPA CLP } \\
\text { EPA CLP } \\
\text { EPA CLP } \\
\text { EPA } 8310 \\
\text { EPA } 8310 \\
\text { EPA 8310 } \\
\text { EPA } 8310 \\
\text { EPA CLP } \\
\text { EPA CLP } \\
\text { EPA CLP } \\
\text { EPA CLP } \\
\text { EPA } 8310 \\
\text { EPA } 8310 \\
\end{array}$ \\
\hline
\end{tabular}




\begin{tabular}{|c|c|c|c|c|c|c|}
\hline WSSRAP_ID & DATE_SAM & PARAMETER & CONC & DL & UNITS & METHOD \\
\hline 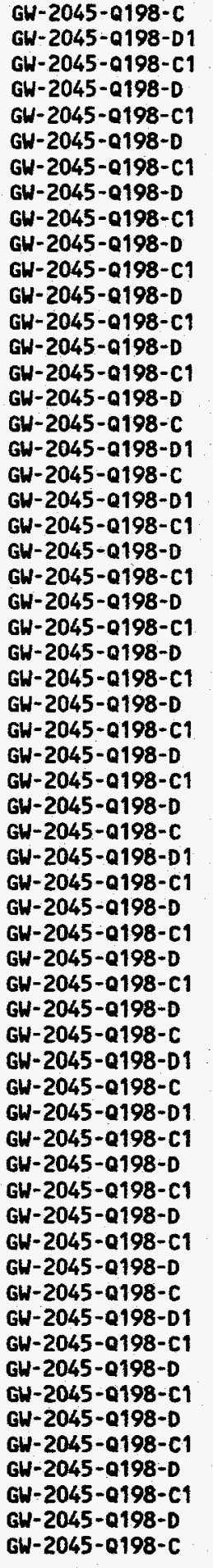 & $\begin{array}{l}01 / 27 / 98 \\
02 / 02 / 98 \\
01 / 28 / 98 \\
01 / 29 / 98 \\
01 / 28 / 98 \\
01 / 29 / 98 \\
01 / 28 / 98 \\
01 / 29 / 98 \\
01 / 28 / 98 \\
01 / 29 / 98 \\
01 / 28 / 98 \\
01 / 29 / 98 \\
01 / 28 / 98 \\
01 / 29 / 98 \\
01 / 28 / 98 \\
01 / 29 / 98 \\
01 / 27 / 98 \\
02 / 02 / 98 \\
01 / 27 / 98 \\
02 / 02 / 98 \\
01 / 28 / 98 \\
01 / 29 / 98 \\
01 / 28 / 98 \\
01 / 29 / 98 \\
01 / 28 / 98 \\
01 / 29 / 98 \\
01 / 28 / 98 \\
01 / 29 / 98 \\
01 / 28 / 98 \\
011 / 29 / 98 \\
01 / 28 / 98 \\
01 / 29 / 98 \\
01 / 27 / 98 \\
02 / 02 / 98 \\
011 / 28 / 98 \\
01 / 29 / 98 \\
01 / 28 / 98 \\
01 / 29 / 98 \\
01 / 28 / 98 \\
01 / 29 / 98 \\
011 / 27 / 98 \\
02 / 02 / 98 \\
01 / 27 / 98 \\
02 / 02 / 98 \\
011 / 28 / 98 \\
01 / 29 / 98 \\
01 / 28 / 98 \\
01 / 29 / 98 \\
011 / 28 / 98 \\
01 / 29 / 98 \\
01 / 27 / 98 \\
02 / 02 / 98 \\
01 / 28 / 98 \\
011 / 29 / 98 \\
01 / 28 / 98 \\
01 / 29 / 98 \\
01 / 28 / 98 \\
01 / 29 / 98 \\
011 / 28 / 98 \\
01 / 29 / 98 \\
01 / 27 / 98\end{array}$ & 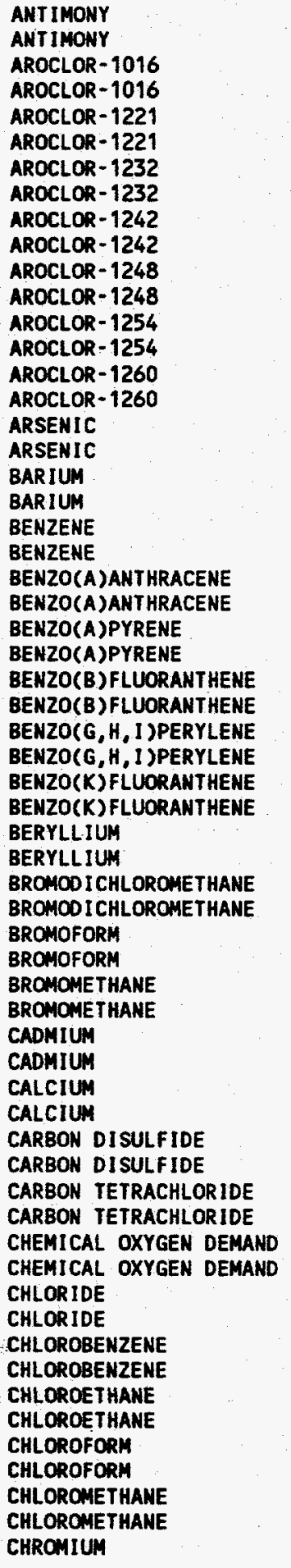 & $\begin{array}{l}\text { ND } \\
\text { ND } \\
\text { ND } \\
\text { ND } \\
\text { ND } \\
\text { ND } \\
\text { ND } \\
\text { ND } \\
\text { ND } \\
\text { ND } \\
\text { ND } \\
\text { ND } \\
\text { ND } \\
\text { ND } \\
\text { 198 } \\
\text { 188 } \\
\text { ND } \\
\text { ND } \\
\text { ND } \\
\text { ND } \\
\text { ND } \\
\text { ND } \\
\text { ND } \\
\text { ND } \\
\text { ND } \\
\text { ND } \\
\text { ND } \\
\text { ND } \\
\text { O. } \\
0.53 \\
\text { ND } \\
\text { ND } \\
\text { ND } \\
\text { ND } \\
\text { ND } \\
\text { ND } \\
\text { ND } \\
\text { ND } \\
10500 \\
\text { NB } \\
\text { ND } \\
\text { ND } \\
\text { ND } \\
\text { ND } \\
\text { ND } \\
\text { ND } \\
16.0 \\
60.5 \\
\text { ND } \\
\text { NO }\end{array}$ & $\begin{array}{l}42.5 \\
42.5 \\
1.0 \\
1.0 \\
1.0 \\
1.0 \\
1.0 \\
1.0 \\
1.0 \\
1.0 \\
1.0 \\
1.0 \\
1.0 \\
1.0 \\
1.0 \\
1.0 \\
1.3 \\
1.3 \\
0.40 \\
0.40 \\
10 \\
20 \\
5.0 \\
5.0 \\
5.0 \\
5.0 \\
5.0 \\
5.0 \\
5.0 \\
5.0 \\
5.0 \\
5.0 \\
0.40 \\
0.40 \\
10 \\
20 \\
10 \\
20 \\
10 \\
20 \\
20 \\
20 \\
20 \\
10 \\
3.0 \\
3.3 \\
3.3 \\
15.9 \\
15.9 \\
10 \\
20 \\
10 \\
20 \\
5.00 \\
5.00 \\
4.00 \\
\end{array}$ & $\begin{array}{l}U G / L \\
U G / L \\
U G / L \\
U G / L \\
U G / L \\
U G / L \\
U G / L \\
U G / L \\
U G / L \\
U G / L \\
U G / L \\
U G / L \\
U G / L \\
U G / L \\
U G / L \\
U G / L \\
U G / L \\
U G / L \\
U G / L \\
U G / L \\
U G / L \\
U G / L \\
U G / L \\
U G / L \\
U G / L \\
U G / L \\
U G / L \\
U G / L \\
U G / L \\
U G / L \\
U G / L \\
U G / L \\
U G / L \\
U G / L \\
U G / L \\
U G / L \\
U G / L \\
U G / L \\
U G / L \\
U G / L \\
U G / L \\
U G / L \\
U G / L \\
U G / L \\
U G / L \\
U G / L \\
U G / L \\
U G / L \\
M G / L \\
U G / L \\
M G / L \\
U G / L \\
U G / L \\
U G / L \\
U G / L \\
U G / L \\
U G / L \\
U G / L \\
U G / L \\
U G / L \\
U G / L \\
U G\end{array}$ & 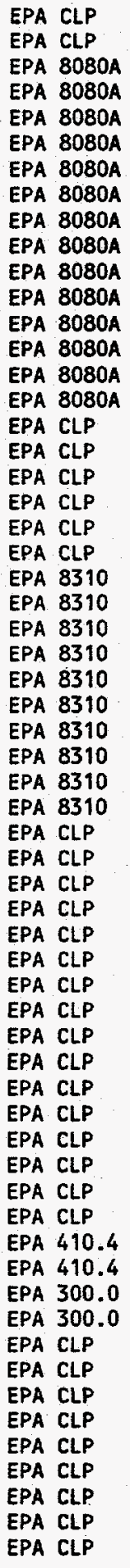 \\
\hline
\end{tabular}




\begin{tabular}{|c|c|c|c|c|c|c|}
\hline WSSRAP_ID & DATE_SAM & PARAMETER & CONC & DL & UNITS & METHOD \\
\hline $\begin{array}{l}G W-2045-Q 198-D 1 \\
G W-2045-0198-C 1 \\
G H-2045-0198-D \\
G W-2045-0198-C 1 \\
G W-2045-0198-D \\
G W-2045-0198-C \\
G W-2045-0198-D 1 \\
G W-2045-0198-C \\
G W-2045-0198-D 1 \\
G W-2045-0198-C \\
G W-2045-0198-D 1 \\
G W-2045-0198-C 1 \\
G W-2045-0198-D \\
G W-2045-0198-C 1 \\
G W-2045-0198-D \\
G W-2045-0198-C 1 \\
G W-2045-0198-D \\
G W-2045-0198-C 1 \\
G W-2045-0198-D \\
G W-2045-0198-C 1 \\
G W-2045-0198-D \\
G H-2045-0198-C \\
G W-2045-0198-D 1 \\
G W-2045-0198-C 1 \\
G W-2045-0198-D \\
G W-2045-0198-C \\
G W-2045-0198-D 1 \\
G W-2045-0198-C \\
G W-2045-0198-D 1 \\
G W-2045-0198-C \\
G W-2045-0198-D 1 \\
G W-2045-0198-C \\
G W-2045-0198-D 1 \\
G W-2045-0198-C \\
G W-2045-0198-D 1 \\
G W-2045-0198-C \\
G W-2045-0198-D 1 \\
G W-2045-0198-C 1 \\
G W-2045-0198-D \\
G W-2045-0198-C \\
G W-2045-0198-D 1 \\
G W-2045-0198-C 1 \\
G W-2045-0198-D \\
G W-2045-0198-C \\
G W-2045-0198-D 1 \\
G W-2045-0198-C 1 \\
G W-2045-0198-D \\
G W-2045-0198-A \\
G W-2045-0198-B \\
G W-2045-0198-D \\
G W-2045-0298-B \\
G W-2045-0298-C \\
G W-2045-0198-C 1 \\
G W-2045-0198-D \\
G W-2045-0198-C \\
G W-2045-0198-D 1 \\
G W-2045-0198-C 1 \\
G W-2045-0198-D \\
G W-2045-0198-C \\
G W-2045-0198-D 1 \\
G W-2045-0198-C \\
\end{array}$ & $\begin{array}{l}02 / 02 / 98 \\
01 / 28 / 98 \\
01 / 29 / 98 \\
01 / 28 / 98 \\
01 / 29 / 98 \\
01 / 27 / 98 \\
02 / 02 / 98 \\
01 / 27 / 98 \\
02 / 02 / 98 \\
01 / 27 / 98 \\
02 / 02 / 98 \\
01 / 28 / 98 \\
01 / 29 / 98 \\
01 / 28 / 98 \\
01 / 29 / 98 \\
01 / 28 / 98 \\
01 / 29 / 98 \\
01 / 28 / 98 \\
01 / 29 / 98 \\
01 / 28 / 98 \\
01 / 29 / 98 \\
01 / 27 / 98 \\
02 / 02 / 98 \\
01 / 28 / 98 \\
01 / 29 / 98 \\
01 / 27 / 98 \\
02 / 02 / 98 \\
01 / 27 / 98 \\
02 / 02 / 98 \\
01 / 27 / 98 \\
02 / 02 / 98 \\
01 / 27 / 98 \\
02 / 02 / 98 \\
01 / 27 / 98 \\
02 / 02 / 98 \\
01 / 27 / 98 \\
02 / 02 / 98 \\
01 / 28 / 98 \\
01 / 29 / 98 \\
01 / 27 / 98 \\
02 / 02 / 98 \\
01 / 28 / 98 \\
01 / 29 / 98 \\
01 / 27 / 98 \\
02 / 02 / 98 \\
01 / 28 / 98 \\
01 / 29 / 98 \\
01 / 21 / 98 \\
01 / 23 / 98 \\
01 / 29 / 98 \\
04 / 08 / 98 \\
04 / 10 / 98 \\
01 / 28 / 98 \\
01 / 29 / 98 \\
01 / 27 / 98 \\
02 / 02 / 98 \\
01 / 28 / 98 \\
01 / 29 / 98 \\
01 / 27 / 98 \\
02 / 02 / 98 \\
01 / 27 / 98\end{array}$ & 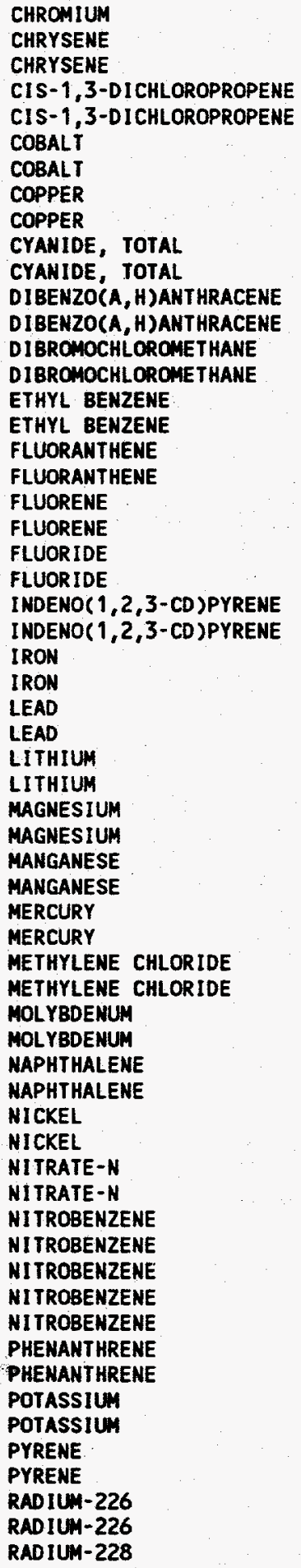 & 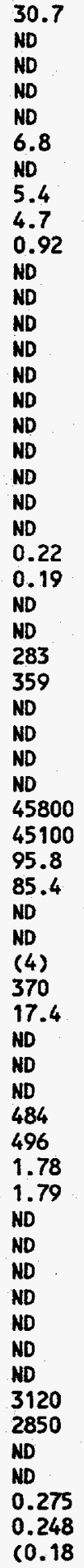 & $\begin{array}{l}2.7 \\
5.0 \\
5.0 \\
10 \\
20 \\
4.3 \\
4.3 \\
4.1 \\
4.1 \\
0.55 \\
0.55 \\
5.0 \\
5.0 \\
10 \\
20 \\
10 \\
20 \\
5.0 \\
5.0 \\
5.0 \\
5.0 \\
0.10 \\
0.10 \\
5.0 \\
5.0 \\
5.9 \\
5.9 \\
0.90 \\
0.90 \\
4.1 \\
4.1 \\
54.5 \\
54.5 \\
1.0 \\
1.0 \\
0.10 \\
0.10 \\
10 \\
20 \\
11.1 \\
11.1 \\
5.0 \\
5.0 \\
14.6 \\
14.6 \\
0.25 \\
0.50 \\
0.030 \\
0.030 \\
0.030 \\
0.030 \\
0.030 \\
5.0 \\
5.0 \\
2180 \\
2180 \\
5.0 \\
5.0 \\
0.032 \\
0.040 \\
0.322\end{array}$ & $\begin{array}{l}U G / L \\
U G / L \\
U G / L \\
U G / L \\
U G / L \\
U G / L \\
U G / L \\
U G / L \\
U G / L \\
U G / L \\
U G / L \\
U G / L \\
U G / L \\
U G / L \\
U G / L \\
U G / L \\
U G / L \\
U G / L \\
U G / L \\
U G / L \\
U G / L \\
M G / L \\
M G / L \\
U G / L \\
U G / L \\
U G / L \\
U G / L \\
U G / L \\
U G / L \\
U G / L \\
U G / L \\
U G / L \\
U G / L \\
U G / L \\
U G / L \\
U G / L \\
U G / L \\
U G / L \\
U G / L \\
U G / L \\
U G / L \\
U G / L \\
U G / L \\
U G / L \\
U G / L \\
M G / L \\
U G / L \\
U G / L \\
U G / L \\
U G / L \\
U G / L \\
U G / L \\
U G / L \\
U G / L \\
U G / L \\
U G / L \\
U G / L \\
P C I / L \\
P C I / L \\
\text { U. }\end{array}$ & 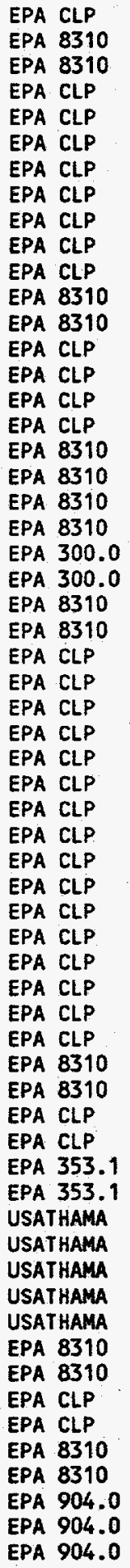 \\
\hline
\end{tabular}




\begin{tabular}{|c|c|c|c|c|c|c|}
\hline WSSRAP_ID & DATE_.SAM & PARAMETER & CONC & $D L$ & UNITS & METHOD \\
\hline 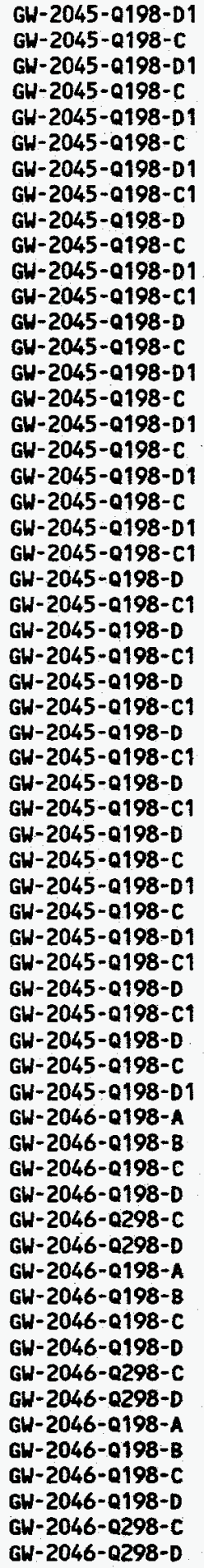 & $\begin{array}{l}02 / 02 / 98 \\
01 / 27 / 98 \\
02 / 02 / 98 \\
01 / 27 / 98 \\
02 / 02 / 98 \\
01 / 27 / 98 \\
02 / 02 / 98 \\
01 / 28 / 98 \\
01 / 29 / 98 \\
01 / 27 / 98 \\
02 / 02 / 98 \\
01 / 28 / 98 \\
01 / 29 / 98 \\
01 / 27 / 98 \\
02 / 02 / 98 \\
01 / 27 / 98 \\
02 / 02 / 98 \\
01 / 27 / 98 \\
02 / 02 / 98 \\
01 / 27 / 98 \\
02 / 02 / 98 \\
01 / 28 / 98 \\
01 / 29 / 98 \\
01 / 28 / 98 \\
01 / 29 / 98 \\
01 / 28 / 98 \\
01 / 29 / 98 \\
01 / 28 / 98 \\
01 / 29 / 98 \\
01 / 28 / 98 \\
01 / 29 / 98 \\
01 / 28 / 98 \\
01 / 29 / 98 \\
01 / 27 / 98 \\
02 / 02 / 98 \\
01 / 27 / 98 \\
02 / 02 / 98 \\
01 / 28 / 98 \\
01 / 29 / 98 \\
01 / 28 / 98 \\
01 / 29 / 98 \\
01 / 27 / 98 \\
02 / 02 / 98 \\
01 / 21 / 98 \\
01 / 22 / 98 \\
01 / 23 / 98 \\
01 / 26 / 98 \\
04 / 08 / 98 \\
04 / 09 / 98 \\
01 / 21 / 98 \\
01 / 22 / 98 \\
01 / 23 / 98 \\
01 / 26 / 98 \\
04 / 08 / 98 \\
04 / 09 / 98 \\
01 / 21 / 98 \\
01 / 22 / 98 \\
01 / 23 / 98 \\
01 / 26 / 98 \\
04 / 08 / 98 \\
04 / 09 / 98\end{array}$ & 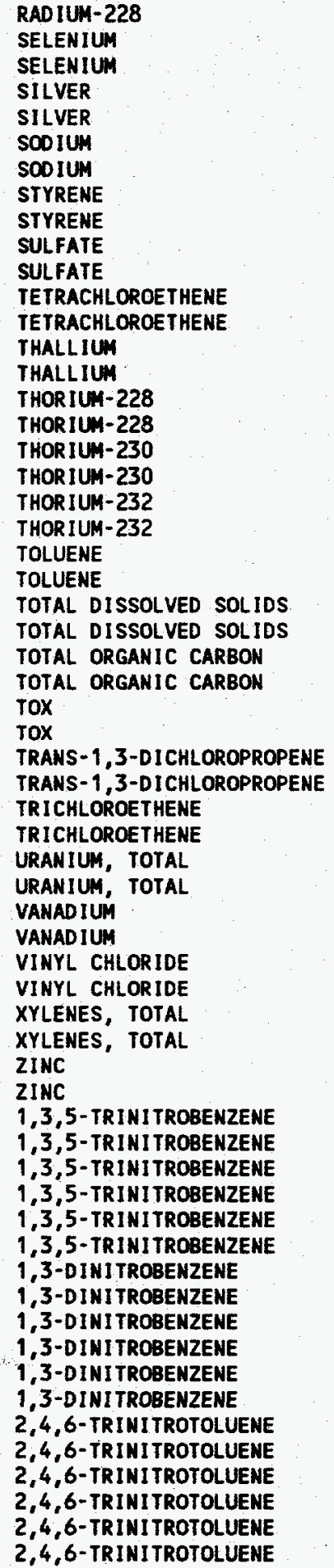 & 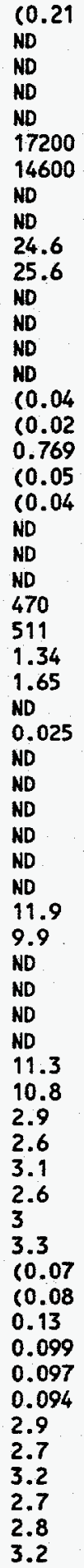 & 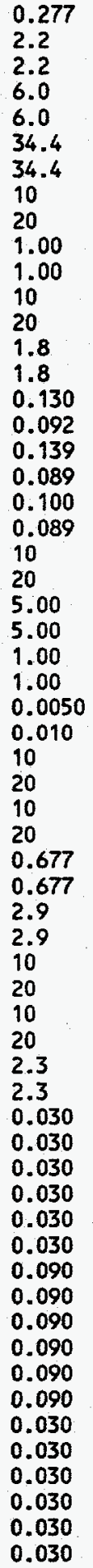 & $\begin{array}{l}P C I / L \\
U G / L \\
U G / L \\
U G / L \\
U G / L \\
U G / L \\
U G / L \\
U G / L \\
U G / L \\
M G / L \\
M G / L \\
U G / L \\
U G / L \\
U G / L \\
U G / L \\
P C I / L \\
P C I / L \\
P C I / L \\
P C I / L \\
P C I / L \\
P C I / L \\
U G / L \\
U G / L \\
M G / L \\
M G / L \\
M G / L \\
M G / L \\
M G / L \\
M G / L \\
U G / L \\
U G / L \\
U G / L \\
U G / L \\
P C I / L \\
P C I / L \\
U G / L \\
U G / L \\
U G / L \\
U G / L \\
U G / L \\
U G / L \\
U G / L \\
U G / L \\
U G / L \\
U G / L \\
U G / L \\
U G / L \\
U G / L \\
U G / L \\
U G / L \\
U G / L \\
U G / L \\
U G / L \\
U G / L \\
U G / L \\
U G / L \\
U G / L \\
U G / L \\
U G / L \\
U G / L \\
U G / L \\
\text { US }\end{array}$ & $\begin{array}{l}\text { EPA } 904.0 \\
\text { EPA CLP } \\
\text { EPA CLP } \\
\text { EPA CLP } \\
\text { EPA CLP } \\
\text { EPA CLP } \\
\text { EPA CLP } \\
\text { EPA CLP } \\
\text { EPA CLP } \\
\text { EPA } 300.0 \\
\text { EPA } 300.0 \\
\text { EPA CLP } \\
\text { EPA CLP } \\
\text { EPA CLP } \\
\text { EPA CLP } \\
\text { NAS-NS-3004 } \\
\text { NAS-NS-3004 } \\
\text { NAS-NS-3004 } \\
\text { NAS-NS-3004 } \\
\text { NAS-NS-3004 } \\
\text { NAS-NS-3004 } \\
\text { EPA CLP } \\
\text { EPA CLP } \\
\text { EPA } 160.1 \\
\text { EPA } 160.1 \\
\text { EPA } 415.1 \\
\text { EPA } 415.1 \\
\text { EPA } 9020 \\
\text { EPA 9020 } \\
\text { EPA CLP } \\
\text { EPA CLP } \\
\text { EPA CLP } \\
\text { EPA CLP } \\
\text { ASTM } 5174-91 \\
\text { ASTM } 5174-91 \\
\text { EPA CLP } \\
\text { EPA CLP } \\
\text { EPA CLP } \\
\text { EPA CLP } \\
\text { EPA CLP } \\
\text { EPA CLP } \\
\text { EPA CLP } \\
\text { EPA CLP } \\
\text { USATHAMA } \\
\text { USATHAMA } \\
\text { USATHAMA } \\
\text { USATHAMA } \\
\text { USATHAMA } \\
\text { USATHAMA } \\
\text { USATHAMA } \\
\text { USATHAMA } \\
\text { USATHAMA } \\
\text { USATHAMA } \\
\text { USATHAMA } \\
\text { USATHAMA } \\
\text { USATHAMA } \\
\text { USATHAMA } \\
\text { USATHAMA } \\
\text { USATHAMA } \\
\text { USATHAMA } \\
\text { USATHAMA } \\
\text { USA }\end{array}$ \\
\hline
\end{tabular}




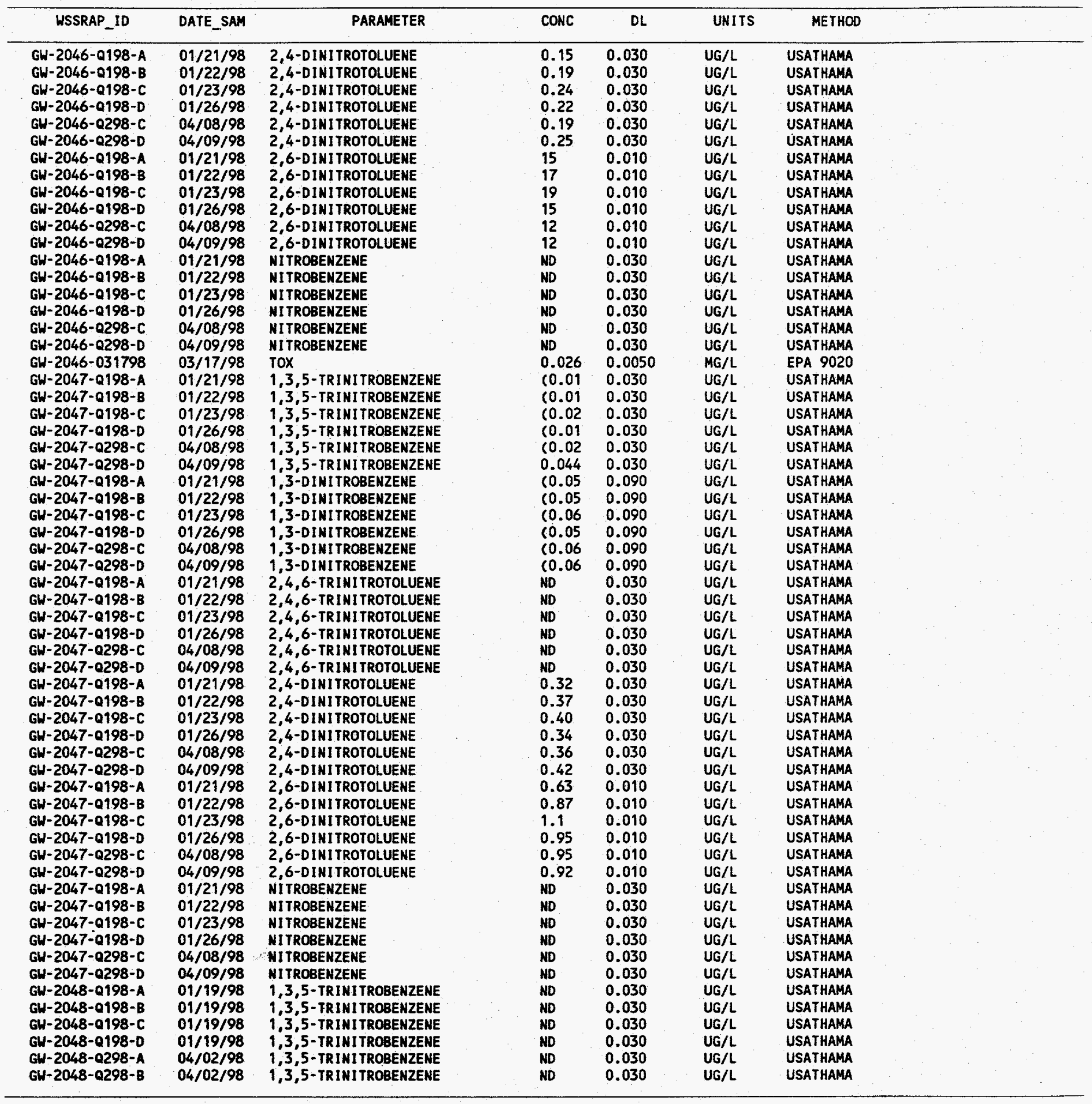




\begin{tabular}{|c|c|c|c|c|c|c|}
\hline WSSRAP ID & DATE_SAM & PARAMETER. & CONC & $\mathrm{DL}$ & UNITS & METHOD \\
\hline 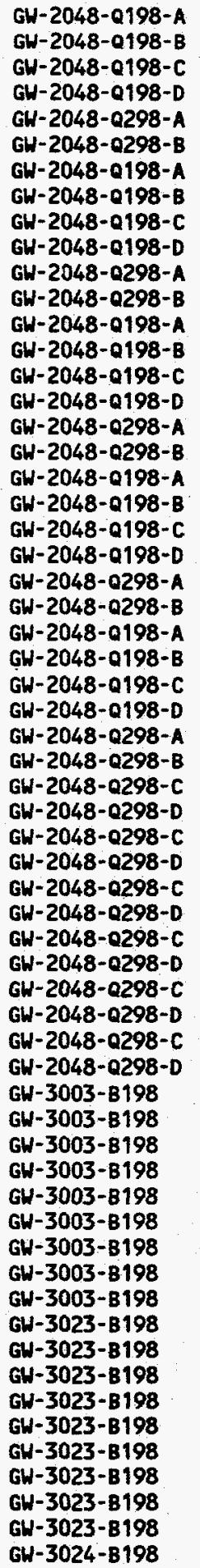 & $\begin{array}{l}01 / 19 / 98 \\
01 / 19 / 98 \\
01 / 19 / 98 \\
01 / 19 / 98 \\
04 / 02 / 98 \\
04 / 02 / 98 \\
01 / 19 / 98 \\
01 / 19 / 98 \\
01 / 19 / 98 \\
01 / 19 / 98 \\
04 / 02 / 98 \\
04 / 02 / 98 \\
01 / 19 / 98 \\
01 / 19 / 98 \\
01 / 19 / 98 \\
01 / 19 / 98 \\
04 / 02 / 98 \\
04 / 02 / 98 \\
01 / 19 / 98 \\
01 / 19 / 98 \\
01 / 19 / 98 \\
01 / 19 / 98 \\
04 / 02 / 98 \\
04 / 02 / 98 \\
01 / 19 / 98 \\
01 / 19 / 98 \\
01 / 19 / 98 \\
01 / 19 / 98 \\
04 / 02 / 98 \\
04 / 02 / 98 \\
04 / 02 / 98 \\
04 / 02 / 98 \\
04 / 02 / 98 \\
04 / 02 / 98 \\
04 / 02 / 98 \\
04 / 02 / 98 \\
04 / 02 / 98 \\
04 / 02 / 98 \\
04 / 02 / 98 \\
04 / 02 / 98 \\
04 / 02 / 98 \\
04 / 02 / 98 \\
02 / 18 / 98 \\
02 / 18 / 98 \\
02 / 18 / 98 \\
02 / 18 / 98 \\
02 / 18 / 98 \\
02 / 18 / 98 \\
02 / 18 / 98 \\
02 / 18 / 98 \\
02 / 18 / 98 \\
02 / 18 / 98 \\
02 / 18 / 98 \\
02 / 18 / 98 \\
02 / 18 / 98 \\
02 / 18 / 98 \\
02 / 18 / 98 \\
02 / 18 / 98 \\
02 / 18 / 98 \\
02 / 18 / 98 \\
01 / 28 / 98\end{array}$ & 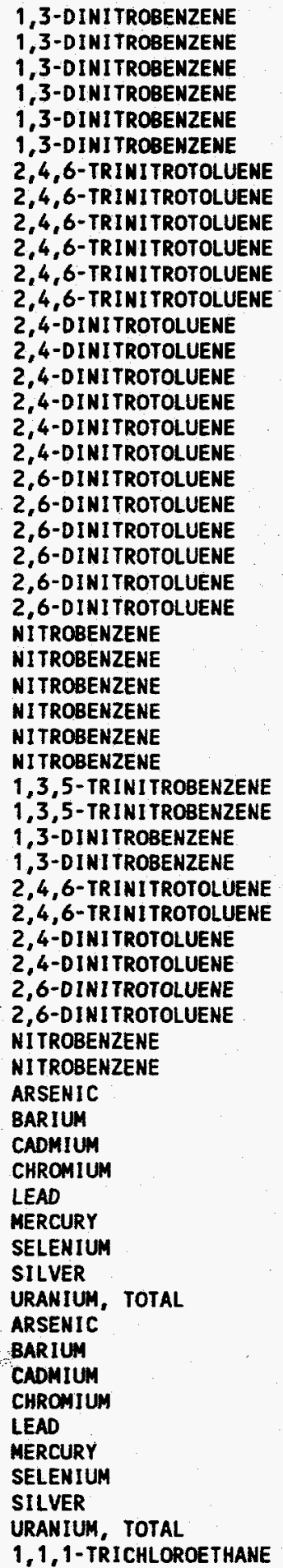 & 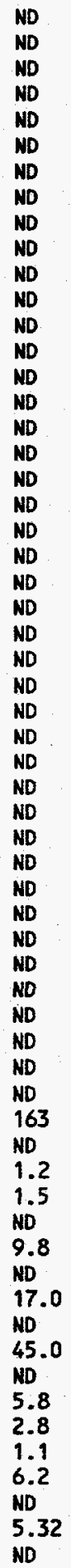 & $\begin{array}{l}0.090 \\
0.090 \\
0.090 \\
0.090 \\
0.090 \\
0.090 \\
0.030 \\
0.030 \\
0.030 \\
0.030 \\
0.030 \\
0.030 \\
0.030 \\
0.030 \\
0.030 \\
0.030 \\
0.030 \\
0.030 \\
0.010 \\
0.010 \\
0.010 \\
0.010 \\
0.010 \\
0.010 \\
0.030 \\
0.030 \\
0.030 \\
0.030 \\
0.030 \\
0.030 \\
0.030 \\
0.030 \\
0.090 \\
0.090 \\
0.030 \\
0.030 \\
0.030 \\
0.030 \\
0.010 \\
0.010 \\
0.030 \\
0.030 \\
1.3 \\
0.40 \\
0.40 \\
0.60 \\
0.90 \\
0.10 \\
2.2 \\
0.80 \\
0.677 \\
1.3 \\
0.40 \\
0.40 \\
0.60 \\
0.90 \\
0.10 \\
2.2 \\
0.80 \\
0.677 \\
10\end{array}$ & $\begin{array}{l}U G / L \\
U G / L \\
U G / L \\
U G / L \\
U G / L \\
U G / L \\
U G / L \\
U G / L \\
U G / L \\
U G / L \\
U G / L \\
U G / L \\
U G / L \\
U G / L \\
U G / L \\
U G / L \\
U G / L \\
U G / L \\
U G / L \\
U G / L \\
U G / L \\
U G / L \\
U G / L \\
U G / L \\
U G / L \\
U G / L \\
U G / L \\
U G / L \\
U G / L \\
U G / L \\
U G / L \\
U G / L \\
U G / L \\
U G / L \\
U G / L \\
U G / L \\
U G / L \\
U G / L \\
U G / L \\
U G / L \\
U G / L \\
U G / L \\
U G / L \\
U G / L \\
U G / L \\
U G / L \\
U G / L \\
U G / L \\
U G / L \\
U G / L \\
P C I / L \\
U G / L \\
U G / L \\
U G / L \\
U G / L \\
U G / L \\
U G / L \\
U G / L \\
U G / L \\
P C I / L \\
U G / L\end{array}$ & $\begin{array}{l}\text { USATHAMA } \\
\text { USATHAMA } \\
\text { USATHAMA } \\
\text { USATHAMA } \\
\text { USATHAMA } \\
\text { USATHAMA } \\
\text { USATHAMA } \\
\text { USATHAMA } \\
\text { USATHAMA } \\
\text { USATHAMA } \\
\text { USATHAMA } \\
\text { USATHAMA } \\
\text { USATHAMA } \\
\text { USATHAMA } \\
\text { USATHAMA } \\
\text { USATHAMA } \\
\text { USATHAMA } \\
\text { USATHAMA } \\
\text { USATHAMA } \\
\text { USATHAMA } \\
\text { USATHAMA } \\
\text { USATHAMA } \\
\text { USATHAMA } \\
\text { USATHAMA } \\
\text { USATHAMA } \\
\text { USATHAMA } \\
\text { USATHAMA } \\
\text { USATHAMA } \\
\text { USATHAMA } \\
\text { USATHAMA } \\
\text { USATHAMA } \\
\text { USATHAMA } \\
\text { USATHAMA } \\
\text { USATHAMA } \\
\text { USATHAMA } \\
\text { USATHAMA } \\
\text { USATHAMA } \\
\text { USATHAMA } \\
\text { USATHAMA } \\
\text { USATHAMA } \\
\text { USATHAMA } \\
\text { USATHAMA } \\
\text { EPA CLP } \\
\text { EPA CLP } \\
\text { EPA CLP } \\
\text { EPA CLP } \\
\text { EPA CLP } \\
\text { EPA CLP } \\
\text { EPA CLP } \\
\text { EPA CLP } \\
\text { ASTM 5174-91 } \\
\text { EPA CLP } \\
\text { EPA CLP } \\
\text { EPA CLP } \\
\text { EPA CLP } \\
\text { EPA CLP } \\
\text { EPA CLP } \\
\text { EPA CLP } \\
\text { EPA CLP } \\
\text { ASTM 5174-91 } \\
\text { EPA CLP } \\
\end{array}$ \\
\hline
\end{tabular}




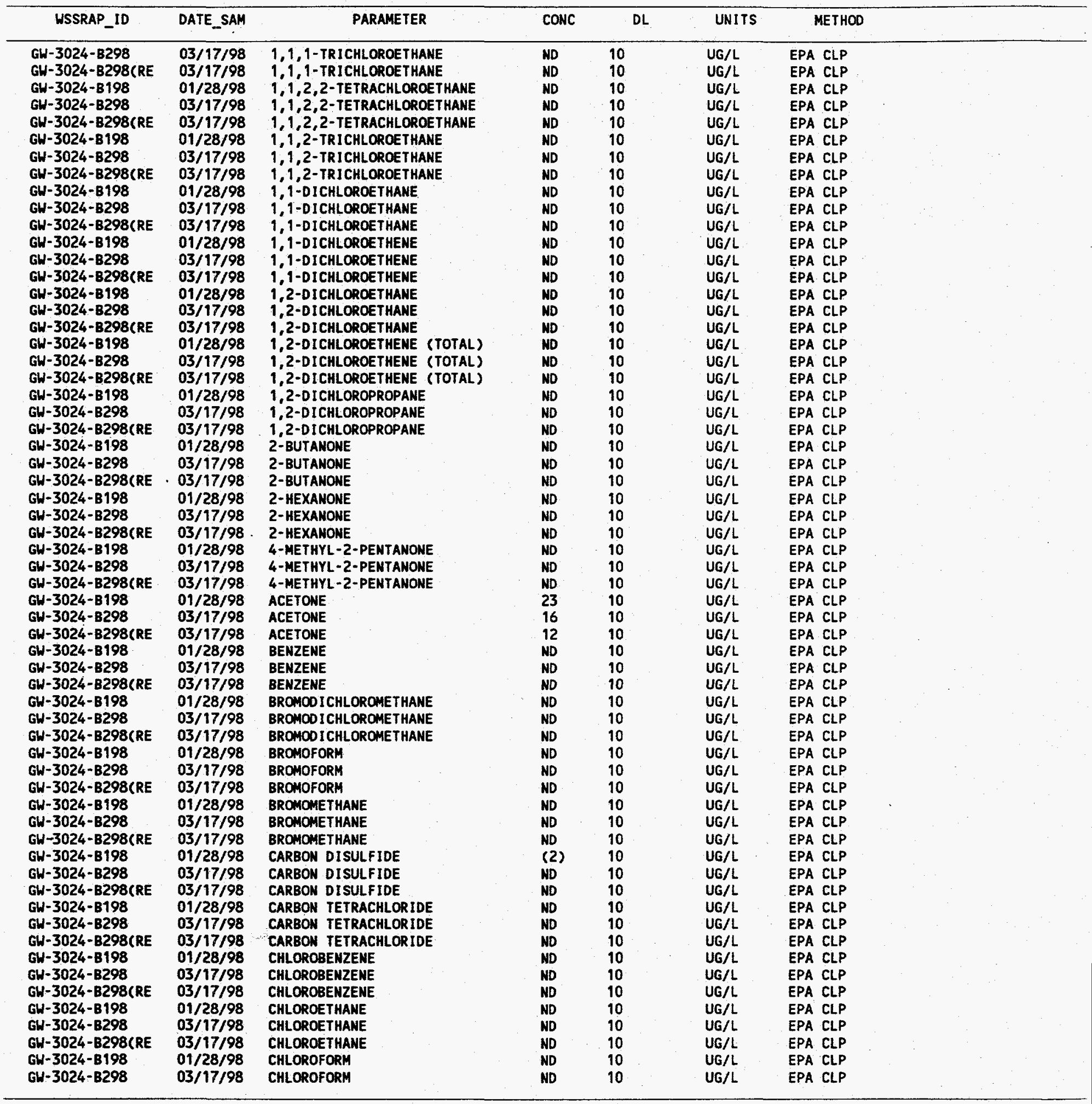




\begin{tabular}{|c|c|c|c|c|c|c|}
\hline WSSRAP_ID & DATE_SAM & PARAMETER & CONC & DL & UNITS & METHOD \\
\hline 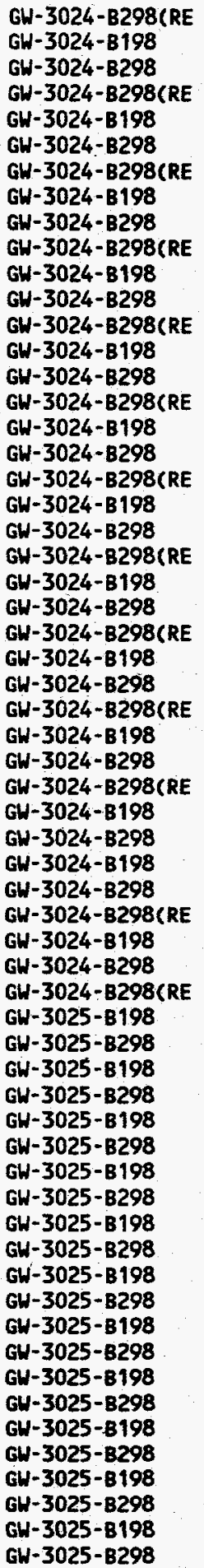 & $\begin{array}{l}03 / 17 / 98 \\
01 / 28 / 98 \\
03 / 17 / 98 \\
03 / 17 / 98 \\
01 / 28 / 98 \\
03 / 17 / 98 \\
03 / 17 / 98 \\
01 / 28 / 98 \\
03 / 17 / 98 \\
03 / 17 / 98 \\
01 / 28 / 98 \\
03 / 17 / 98 \\
03 / 17 / 98 \\
01 / 28 / 98 \\
03 / 17 / 98 \\
03 / 17 / 98 \\
01 / 28 / 98 \\
03 / 17 / 98 \\
03 / 17 / 98 \\
01 / 28 / 98 \\
03 / 17 / 98 \\
03 / 17 / 98 \\
01 / 28 / 98 \\
03 / 17 / 98 \\
03 / 17 / 98 \\
01 / 28 / 98 \\
03 / 17 / 98 \\
03 / 17 / 98 \\
01 / 28 / 98 \\
03 / 17 / 98 \\
03 / 17 / 98 \\
01 / 28 / 98 \\
03 / 17 / 98 \\
01 / 28 / 98 \\
03 / 17 / 98 \\
03 / 17 / 98 \\
01 / 28 / 98 \\
03 / 17 / 98 \\
03 / 17 / 98 \\
01 / 28 / 98 \\
03 / 10 / 98 \\
01 / 28 / 98 \\
03 / 10 / 98 \\
01 / 28 / 98 \\
03 / 10 / 98 \\
01 / 28 / 98 \\
03 / 10 / 98 \\
01 / 28 / 98 \\
03 / 10 / 98 \\
01 / 28 / 98 \\
03 / 10 / 98 \\
01 / 28 / 98 \\
03 / 10 / 98 \\
01 / 28 / 98 \\
03 / 10 / 98 \\
01 / 28 / 98 \\
03 / 10 / 98 \\
01 / 28 / 98 \\
03 / 10 / 98 \\
01 / 28 / 98 \\
03 / 10 / 98\end{array}$ & 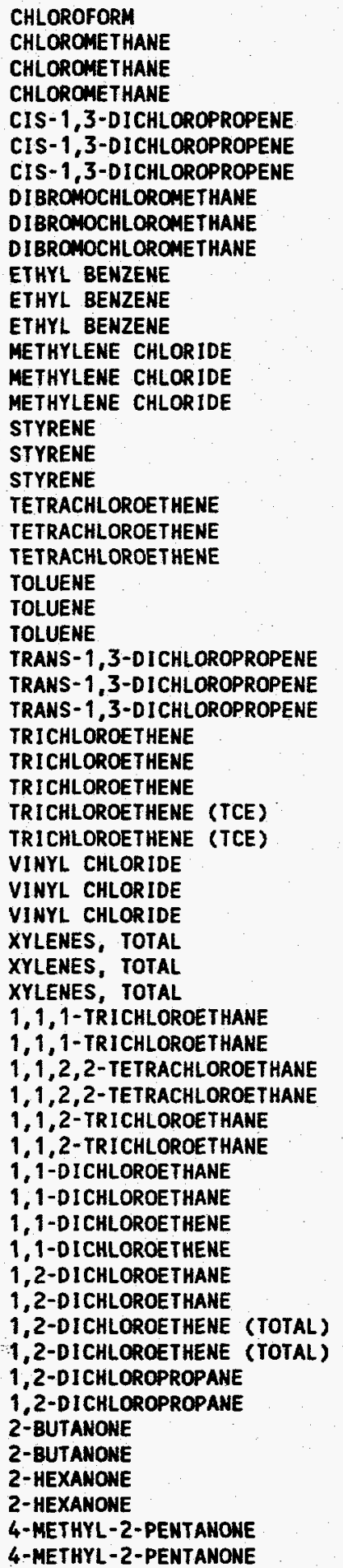 & $\begin{array}{l}\text { ND } \\
\text { ND } \\
\text { ND } \\
\text { ND } \\
\text { ND } \\
\text { ND } \\
\text { ND } \\
\text { ND } \\
\text { ND } \\
\text { ND } \\
\text { ND } \\
\text { ND } \\
\text { ND } \\
(4) \\
\text { (3) } \\
\text { C5) } \\
\text { ND } \\
\text { ND } \\
\text { ND } \\
\text { ND } \\
\text { ND } \\
\text { ND } \\
(2) \\
\text { (1) } \\
\text { (1) } \\
\text { ND } \\
\text { ND } \\
\text { ND } \\
\text { ND } \\
\text { ND } \\
\text { ND } \\
\text { ND } \\
\text { ND } \\
\text { ND } \\
\text { ND } \\
\text { ND } \\
\text { ND } \\
\text { ND } \\
\text { ND } \\
\text { ND } \\
\text { ND } \\
\text { ND } \\
\text { ND } \\
\text { ND } \\
\text { ND } \\
\text { ND } \\
\text { ND } \\
\text { ND } \\
\text { ND } \\
\text { ND } \\
\text { ND } \\
\text { ND } \\
\text { (2) } \\
\text { ND } \\
\text { ND } \\
\text { ND } \\
\text { ND } \\
\text { ND } \\
\text { ND } \\
\text { ND } \\
\text { ND }\end{array}$ & $\begin{array}{l}10 \\
10 \\
10 \\
10 \\
10 \\
10 \\
10 \\
10 \\
10 \\
10 \\
10 \\
10 \\
10 \\
10 \\
10 \\
10 \\
10 \\
10 \\
10 \\
10 \\
10 \\
10 \\
10 \\
10 \\
10 \\
10 \\
10 \\
10 \\
10 \\
10 \\
10 \\
1 \\
1 \\
10 \\
10 \\
10 \\
10 \\
10 \\
10 \\
10 \\
10 \\
10 \\
10 \\
10 \\
10 \\
10 \\
10 \\
10 \\
10 \\
10 \\
10 \\
10 \\
10 \\
10 \\
10 \\
10 \\
10 \\
10 \\
10 \\
10 \\
10\end{array}$ & $\begin{array}{l}U G / L \\
U G / L \\
U G / L \\
U G / L \\
U G / L \\
U G / L \\
U G / L \\
U G / L \\
U G / L \\
U G / L \\
U G / L \\
U G / L \\
U G / L \\
U G / L \\
U G / L \\
U G / L \\
U G / L \\
U G / L \\
U G / L \\
U G / L \\
U G / L \\
U G / L \\
U G / L \\
U G / L \\
U G / L \\
U G / L \\
U G / L \\
U G / L \\
U G / L \\
U G / L \\
U G / L \\
U G / L \\
U G / L \\
U G / L \\
U G / L \\
U G / L \\
U G / L \\
U G / L \\
U G / L \\
U G / L \\
U G / L \\
U G / L \\
U G / L \\
U G / L \\
U G / L \\
U G / L \\
U G / L \\
U G / L \\
U G / L \\
U G / L \\
U G / L \\
U G / L \\
U G / L \\
U G / L \\
U G / L \\
U G / L \\
U G / L \\
U G / L \\
U G / L \\
U G / L \\
U\end{array}$ & $\begin{array}{l}\text { EPA CLP } \\
\text { EPA CLP } \\
\text { EPA CLP } \\
\text { EPA CLP } \\
\text { EPA CLP } \\
\text { EPA CLP } \\
\text { EPA CLP } \\
\text { EPA CLP } \\
\text { EPA CLP } \\
\text { EPA CLP } \\
\text { EPA CLP } \\
\text { EPA CLP } \\
\text { EPA CLP } \\
\text { EPA CLP } \\
\text { EPA CLP } \\
\text { EPA CLP } \\
\text { EPA CLP } \\
\text { EPA CLP } \\
\text { EPA CLP } \\
\text { EPA CLP } \\
\text { EPA CLP } \\
\text { EPA CLP } \\
\text { EPA CLP } \\
\text { EPA CLP } \\
\text { EPA CLP } \\
\text { EPA CLP } \\
\text { EPA CLP } \\
\text { EPA CLP } \\
\text { EPA CLP } \\
\text { EPA CLP } \\
\text { EPA CLP } \\
\text { EPA } 8260 \\
\text { EPA } 8260 \\
\text { EPA CLP } \\
\text { EPA CLP } \\
\text { EPA CLP } \\
\text { EPA CLP } \\
\text { EPA CLP } \\
\text { EPA CLP } \\
\text { EPA CLP } \\
\text { EPA CLP } \\
\text { EPA CLP } \\
\text { EPA CLP } \\
\text { EPA CLP } \\
\text { EPA CLP } \\
\text { EPA CLP } \\
\text { EPA CLP } \\
\text { EPA CLP } \\
\text { EPA CLP } \\
\text { EPA CLP } \\
\text { EPA CLP } \\
\text { EPA CLP } \\
\text { EPA CLP } \\
\text { EPA CLP } \\
\text { EPA CLP } \\
\text { EPA CLP } \\
\text { EPA CLP } \\
\text { EPA CLP } \\
\text { EPA CLP } \\
\text { EPA CLP } \\
\text { EPA CLP }\end{array}$ \\
\hline
\end{tabular}




\begin{tabular}{|c|c|c|c|c|c|c|}
\hline WSSRAP_ID & DATE_SAM & PARAMETER & CONC & $D L$ & UNITS & METHOD \\
\hline 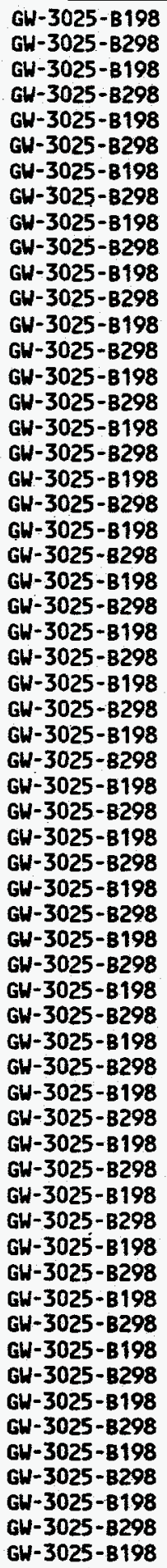 & $\begin{array}{l}01 / 28 / 98 \\
03 / 10 / 98 \\
01 / 28 / 98 \\
03 / 10 / 98 \\
01 / 28 / 98 \\
03 / 10 / 98 \\
01 / 28 / 98 \\
03 / 10 / 98 \\
01 / 28 / 98 \\
03 / 10 / 98 \\
01 / 28 / 98 \\
03 / 10 / 98 \\
01 / 28 / 98 \\
03 / 10 / 98 \\
01 / 28 / 98 \\
03 / 10 / 98 \\
01 / 28 / 98 \\
03 / 10 / 98 \\
01 / 28 / 98 \\
03 / 10 / 98 \\
01 / 28 / 98 \\
03 / 10 / 98 \\
01 / 28 / 98 \\
03 / 10 / 98 \\
01 / 28 / 98 \\
03 / 10 / 98 \\
01 / 28 / 98 \\
03 / 10 / 98 \\
01 / 28 / 98 \\
03 / 10 / 98 \\
01 / 28 / 98 \\
03 / 10 / 98 \\
01 / 28 / 98 \\
03 / 10 / 98 \\
01 / 28 / 98 \\
03 / 10 / 98 \\
01 / 28 / 98 \\
03 / 10 / 98 \\
01 / 28 / 98 \\
03 / 10 / 98 \\
01 / 28 / 98 \\
03 / 10 / 98 \\
01 / 28 / 98 \\
03 / 10 / 98 \\
01 / 28 / 98 \\
03 / 10 / 98 \\
01 / 28 / 98 \\
03 / 10 / 98 \\
01 / 28 / 98 \\
03 / 10 / 98 \\
01 / 28 / 98 \\
03 / 10 / 98 \\
01 / 28 / 98 \\
03 / 10 / 98 \\
01 / 28 / 98 \\
03 / 10 / 98 \\
01 / 28 / 98 \\
03 / 10 / 98 \\
01 / 28 / 98 \\
03 / 10 / 98 \\
01 / 28 / 98\end{array}$ & 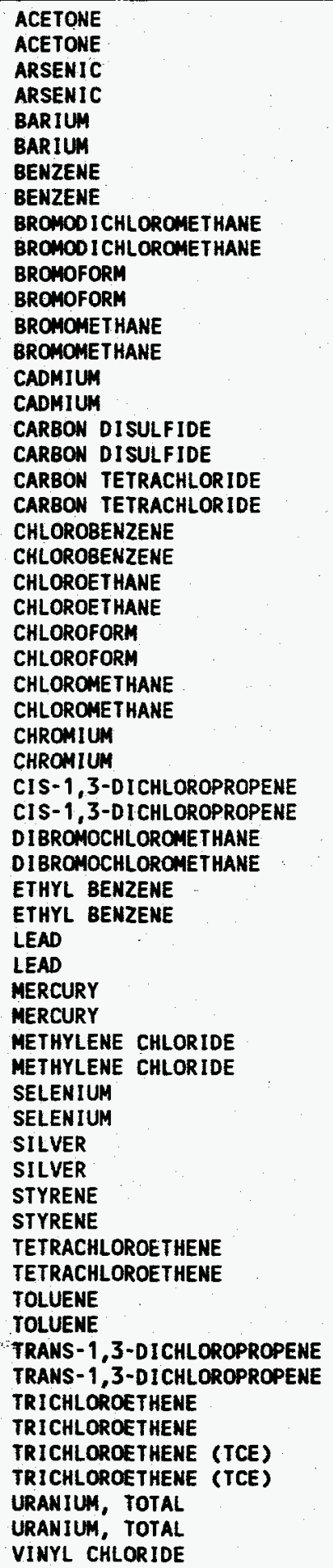 & $\begin{array}{l}(8) \\
\text { (9) } \\
\text { ND } \\
\text { ND } \\
331 \\
311 \\
\text { ND } \\
\text { ND } \\
\text { ND } \\
\text { ND } \\
\text { ND } \\
\text { ND } \\
\text { ND } \\
\text { ND } \\
\text { ND } \\
\text { ND } \\
\text { ND } \\
\text { ND } \\
\text { ND } \\
\text { ND } \\
\text { ND } \\
\text { ND } \\
\text { ND } \\
\text { ND } \\
\text { ND } \\
\text { (2) } \\
\text { ND } \\
\text { ND } \\
\text { ND } \\
1.4 \\
\text { ND } \\
\text { ND } \\
\text { ND } \\
\text { ND } \\
\text { ND } \\
\text { ND } \\
\text { ND } \\
\text { ND } \\
\text { O.13 } \\
\text { ND } \\
(4) \\
(4) \\
10.6 \\
10.2 \\
\text { ND } \\
\text { ND } \\
\text { ND } \\
\text { ND } \\
\text { ND } \\
\text { ND } \\
\text { ND } \\
\text { ND } \\
\text { ND } \\
\text { ND } \\
\text { (5) } \\
\text { 24 } \\
\text { 28 } \\
\text { (0.6) } \\
3.03 \\
3.00 \\
\text { ND }\end{array}$ & $\begin{array}{l}10 \\
10 \\
1.3 \\
1.3 \\
0.40 \\
0.40 \\
10 \\
10 \\
10 \\
10 \\
10 \\
10 \\
10 \\
10 \\
3.3 \\
0.40 \\
10 \\
10 \\
10 \\
10 \\
10 \\
10 \\
10 \\
10 \\
10 \\
10 \\
10 \\
10 \\
2.7 \\
0.60 \\
10 \\
10 \\
10 \\
10 \\
10 \\
10 \\
0.90 \\
0.90 \\
0.10 \\
0.10 \\
10 \\
10 \\
2.2 \\
2.2 \\
6.0 \\
0.80 \\
10 \\
10 \\
10 \\
10 \\
10 \\
10 \\
10 \\
10 \\
10 \\
10 \\
1 \\
1 \\
0.677 \\
0.677 \\
10\end{array}$ & $\begin{array}{l}\text { UG/L } \\
U G / L \\
U G / L \\
U G / L \\
U G / L \\
U G / L \\
U G / L \\
U G / L \\
U G / L \\
U G / L \\
U G / L \\
U G / L \\
U G / L \\
U G / L \\
U G / L \\
U G / L \\
U G / L \\
U G / L \\
U G / L \\
U G / L \\
U G / L \\
U G / L \\
U G / L \\
U G / L \\
U G / L \\
U G / L \\
U G / L \\
U G / L \\
U G / L \\
U G / L \\
U G / L \\
U G / L \\
U G / L \\
U G / L \\
U G / L \\
U G / L \\
U G / L \\
U G / L \\
U G / L \\
U G / L \\
U G / L \\
U G / L \\
U G / L \\
U G / L \\
U G / L \\
U G / L \\
U G / L \\
U G / L \\
U G / L \\
U G / L \\
U G / L \\
U G / L \\
U G / L \\
U G / L \\
U G / L \\
U G / L \\
U G / L \\
U G / L \\
P C I / L \\
P C I / L \\
U G / L \\
\text { US }\end{array}$ & $\begin{array}{l}\text { EPA CLP } \\
\text { EPA CLP } \\
\text { EPA CLP } \\
\text { EPA CLP } \\
\text { EPA CLP } \\
\text { EPA CLP } \\
\text { EPA CLP } \\
\text { EPA CLP } \\
\text { EPA CLP } \\
\text { EPA CLP } \\
\text { EPA CLP } \\
\text { EPA CLP } \\
\text { EPA CLP } \\
\text { EPA CLP } \\
\text { EPA CLP } \\
\text { EPA CLP } \\
\text { EPA CLP } \\
\text { EPA CLP } \\
\text { EPA CLP } \\
\text { EPA CLP } \\
\text { EPA CLP } \\
\text { EPA CLP } \\
\text { EPA CLP } \\
\text { EPA CLP } \\
\text { EPA CLP } \\
\text { EPA CLP } \\
\text { EPA CLP } \\
\text { EPA CLP } \\
\text { EPA CLP } \\
\text { EPA CLP } \\
\text { EPA CLP } \\
\text { EPA CLP } \\
\text { EPA CLP } \\
\text { EPA CLP } \\
\text { EPA CLP } \\
\text { EPA CLP } \\
\text { EPA CLP } \\
\text { EPA CLP } \\
\text { EPA CLP } \\
\text { EPA CLP } \\
\text { EPA CLP } \\
\text { EPA CLP } \\
\text { EPA CLP } \\
\text { EPA CLP } \\
\text { EPA CLP } \\
\text { EPA CLP } \\
\text { EPA CLP } \\
\text { EPA CLP } \\
\text { EPA CLP } \\
\text { EPA CLP } \\
\text { EPA CLP } \\
\text { EPA CLP } \\
\text { EPA CLP } \\
\text { EPA CLP } \\
\text { EPA CLP } \\
\text { EPA CLP } \\
\text { EPA } 8260 \\
\text { EPA } 8260 \\
\text { ASTM } 5174-91 \\
\text { ASTM } 5174-91 \\
\text { EPA CLP } \\
\end{array}$ \\
\hline
\end{tabular}




\begin{tabular}{|c|c|c|c|c|c|c|}
\hline WSSRAP_ID & DATE_SAM & PARAMETER & CONC & $D L$ & UNITS & METHOD \\
\hline 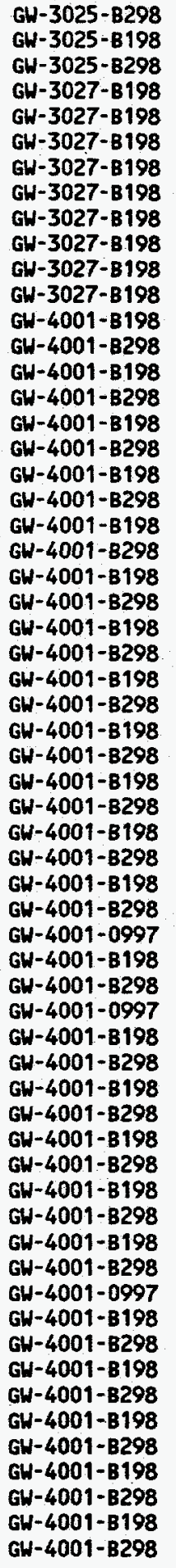 & $\begin{array}{l}03 / 10 / 98 \\
01 / 28 / 98 \\
03 / 10 / 98 \\
02 / 18 / 98 \\
02 / 18 / 98 \\
02 / 18 / 98 \\
02 / 18 / 98 \\
02 / 18 / 98 \\
02 / 18 / 98 \\
02 / 18 / 98 \\
02 / 18 / 98 \\
02 / 18 / 98 \\
01 / 29 / 98 \\
03 / 26 / 98 \\
01 / 29 / 98 \\
03 / 26 / 98 \\
01 / 29 / 98 \\
03 / 26 / 98 \\
01 / 29 / 98 \\
03 / 26 / 98 \\
01 / 29 / 98 \\
03 / 26 / 98 \\
01 / 29 / 98 \\
03 / 26 / 98 \\
01 / 29 / 98 \\
03 / 26 / 98 \\
01 / 29 / 98 \\
03 / 26 / 98 \\
01 / 29 / 98 \\
03 / 26 / 98 \\
01 / 29 / 98 \\
03 / 26 / 98 \\
01 / 29 / 98 \\
03 / 26 / 98 \\
01 / 29 / 98 \\
03 / 26 / 98 \\
09 / 22 / 97 \\
01 / 29 / 98 \\
03 / 26 / 98 \\
09 / 22 / 97 \\
01 / 29 / 98 \\
03 / 26 / 98 \\
01 / 29 / 98 \\
03 / 26 / 98 \\
01 / 29 / 98 \\
03 / 26 / 98 \\
01 / 29 / 98 \\
03 / 26 / 98 \\
01 / 29 / 98 \\
03 / 26 / 98 \\
09 / 22 / 97 \\
01 / 29 / 98 \\
03 / 26 / 98 \\
01 / 29 / 98 \\
03 / 26 / 98 \\
01 / 29 / 98 \\
03 / 26 / 98 \\
01 / 29 / 98 \\
03 / 26 / 98 \\
01 / 29 / 98 \\
03 / 26 / 98\end{array}$ & 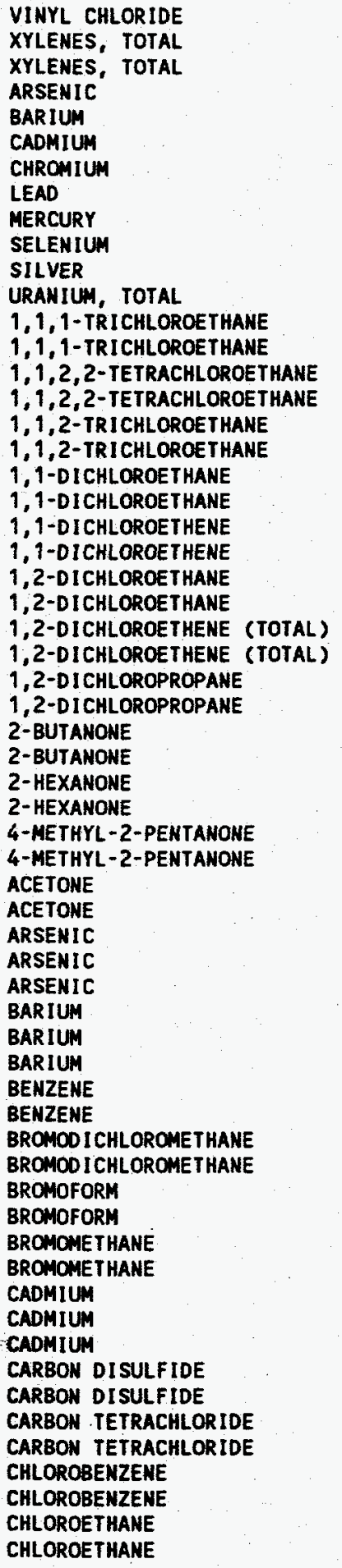 & $\begin{array}{l}\text { ND } \\
\text { ND } \\
\text { ND } \\
\text { ND } \\
751 \\
\text { ND } \\
2.4 \\
2.0 \\
\text { ND } \\
2.3 \\
\text { ND } \\
1.06 \\
\text { ND } \\
\text { ND } \\
\text { ND } \\
\text { ND } \\
\text { ND } \\
\text { ND } \\
\text { ND } \\
\text { ND } \\
\text { ND } \\
\text { ND } \\
\text { ND } \\
\text { ND } \\
\text { ND } \\
\text { ND } \\
\text { ND } \\
\text { ND } \\
\text { ND } \\
\text { ND } \\
\text { ND } \\
\text { ND } \\
\text { ND } \\
\text { ND } \\
\text { (5) } \\
\text { ND } \\
\text { ND } \\
\text { ND } \\
\text { ND } \\
83.8 \\
92.6 \\
97.5 \\
\text { ND } \\
\text { ND } \\
\text { ND } \\
\text { ND } \\
\text { ND } \\
\text { ND } \\
\text { ND } \\
\text { ND } \\
\text { ND } \\
\text { ND } \\
\text { ND } \\
\text { ND } \\
\text { ND } \\
\text { ND } \\
\text { ND } \\
\text { ND } \\
\text { ND } \\
\text { ND }\end{array}$ & $\begin{array}{l}10 \\
10 \\
10 \\
1.3 \\
0.40 \\
0.40 \\
0.60 \\
0.90 \\
0.10 \\
2.2 \\
0.80 \\
0.677 \\
10 \\
10 \\
10 \\
10 \\
10 \\
10 \\
10 \\
10 \\
10 \\
10 \\
10 \\
10 \\
10 \\
10 \\
10 \\
10 \\
10 \\
10 \\
10 \\
10 \\
10 \\
10 \\
10 \\
10 \\
2.00 \\
1.3 \\
1.9 \\
5.00 \\
0.40 \\
1.0 \\
10 \\
10 \\
10 \\
10 \\
10 \\
10 \\
10 \\
10 \\
5.00 \\
0.40 \\
0.60 \\
10 \\
10 \\
10 \\
10 \\
10 \\
10 \\
10 \\
10 \\
\\
10 \\
\end{array}$ & $\begin{array}{l}U G / L \\
U G / L \\
U G / L \\
U G / L \\
U G / L \\
U G / L \\
U G / L \\
U G / L \\
U G / L \\
U G / L \\
U G / L \\
P C I / L \\
U G / L \\
U G / L \\
U G / L \\
U G / L \\
U G / L \\
U G / L \\
U G / L \\
U G / L \\
U G / L \\
U G / L \\
U G / L \\
U G / L \\
U G / L \\
U G / L \\
U G / L \\
U G / L \\
U G / L \\
U G / L \\
U G / L \\
U G / L \\
U G / L \\
U G / L \\
U G / L \\
U G / L \\
U G / L \\
U G / L \\
U G / L \\
U G / L \\
U G / L \\
U G / L \\
U G / L \\
U G / L \\
U G / L \\
U G / L \\
U G / L \\
U G / L \\
U G / L \\
U G / L \\
U G / L \\
U G / L \\
U G / L \\
U G / L \\
U G / L \\
U G / L \\
U G / L \\
U G / L \\
U G / L \\
U G / L \\
U G / L \\
\text { US }\end{array}$ & $\begin{array}{l}\text { EPA CLP } \\
\text { EPA CLP } \\
\text { EPA CLP } \\
\text { EPA CLP } \\
\text { EPA CLP } \\
\text { EPA CLP } \\
\text { EPA CLP } \\
\text { EPA CLP } \\
\text { EPA CLP } \\
\text { EPA CLP } \\
\text { EPA CLP } \\
\text { ASTM } 5174-91 \\
\text { EPA CLP } \\
\text { EPA CLP } \\
\text { EPA CLP } \\
\text { EPA CLP } \\
\text { EPA CLP } \\
\text { EPA CLP } \\
\text { EPA CLP } \\
\text { EPA CLP } \\
\text { EPA CLP } \\
\text { EPA CLP } \\
\text { EPA CLP } \\
\text { EPA CLP } \\
\text { EPA CLP } \\
\text { EPA CLP } \\
\text { EPA CLP } \\
\text { EPA CLP } \\
\text { EPA CLP } \\
\text { EPA CLP } \\
\text { EPA CLP } \\
\text { EPA CLP } \\
\text { EPA CLP } \\
\text { EPA CLP } \\
\text { EPA CLP } \\
\text { EPA CLP } \\
\text { EPA CLP } \\
\text { EPA CLP } \\
\text { EPA CLP } \\
\text { EPA CLP } \\
\text { EPA CLP } \\
\text { EPA CLP } \\
\text { EPA CLP } \\
\text { EPA CLP } \\
\text { EPA CLP } \\
\text { EPA CLP } \\
\text { EPA CLP } \\
\text { EPA CLP } \\
\text { EPA CLP } \\
\text { EPA CLP } \\
\text { EPA CLP } \\
\text { EPA CLP } \\
\text { EPA CLP } \\
\text { EPA CLP } \\
\text { EPA CLP } \\
\text { EPA CLP } \\
\text { EPA CLP } \\
\text { EPA CLP } \\
\text { EPA CLP } \\
\text { EPA CLP } \\
\text { EPA CLP } \\
\end{array}$ \\
\hline
\end{tabular}




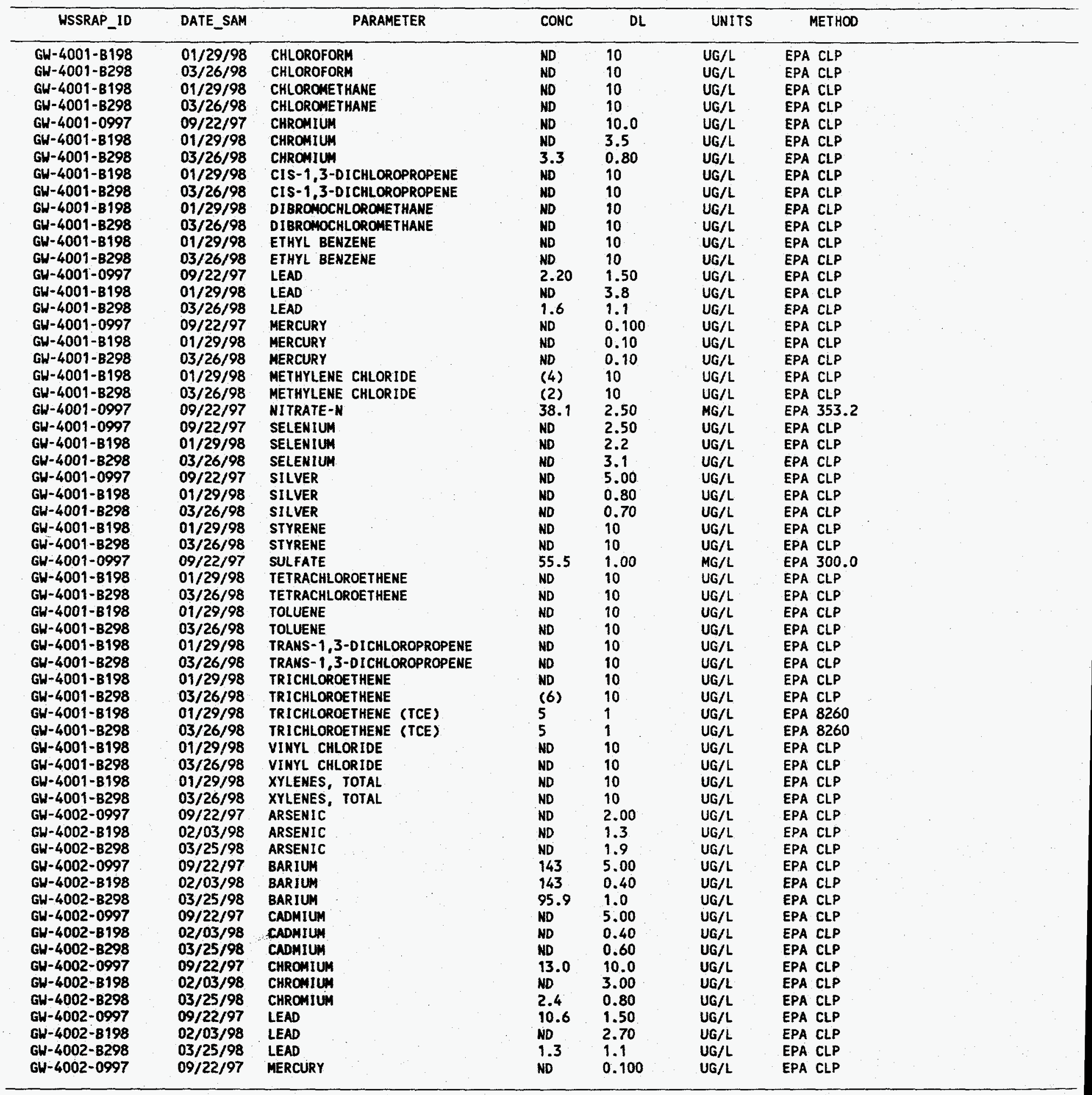




\begin{tabular}{|c|c|c|c|c|c|c|}
\hline WSSRAP_ID & DATE_SAM & PARAMETER & CONC & $\mathrm{DL}$ & UNITS & METHOD \\
\hline 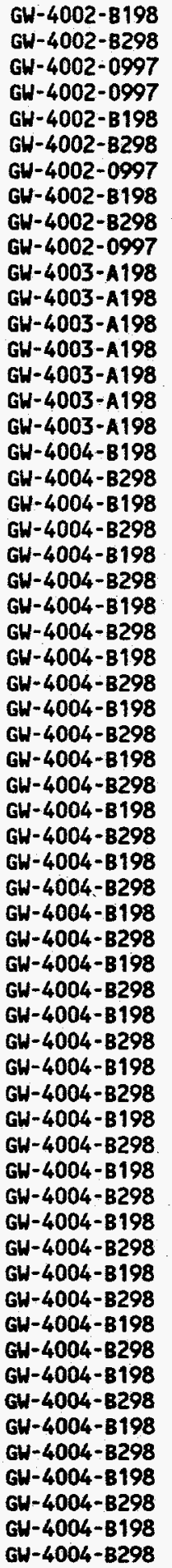 & $\begin{array}{l}02 / 03 / 98 \\
03 / 25 / 98 \\
09 / 22 / 97 \\
09 / 22 / 97 \\
02 / 03 / 98 \\
03 / 25 / 98 \\
09 / 22 / 97 \\
02 / 03 / 98 \\
03 / 25 / 98 \\
09 / 22 / 97 \\
03 / 25 / 98 \\
03 / 25 / 98 \\
03 / 25 / 98 \\
03 / 25 / 98 \\
03 / 25 / 98 \\
03 / 25 / 98 \\
03 / 25 / 98 \\
01 / 29 / 98 \\
03 / 25 / 98 \\
01 / 29 / 98 \\
03 / 25 / 98 \\
01 / 29 / 98 \\
03 / 25 / 98 \\
01 / 29 / 98 \\
03 / 25 / 98 \\
01 / 29 / 98 \\
03 / 25 / 98 \\
01 / 29 / 98 \\
03 / 25 / 98 \\
01 / 29 / 98 \\
03 / 25 / 98 \\
01 / 29 / 98 \\
03 / 25 / 98 \\
01 / 29 / 98 \\
03 / 25 / 98 \\
01 / 29 / 98 \\
03 / 25 / 98 \\
01 / 29 / 98 \\
03 / 25 / 98 \\
01 / 29 / 98 \\
03 / 25 / 98 \\
01 / 29 / 98 \\
03 / 25 / 98 \\
01 / 29 / 98 \\
03 / 25 / 98 \\
01 / 29 / 98 \\
03 / 25 / 98 \\
01 / 29 / 98 \\
03 / 25 / 98 \\
01 / 29 / 98 \\
03 / 25 / 98 \\
01 / 29 / 98 \\
03 / 25 / 98 \\
01 / 29 / 98 \\
03 / 25 / 98 \\
01 / 29 / 98 \\
03 / 25 / 98 \\
01 / 29 / 98 \\
03 / 25 / 98 \\
01 / 29 / 98 \\
03 / 25 / 98\end{array}$ & 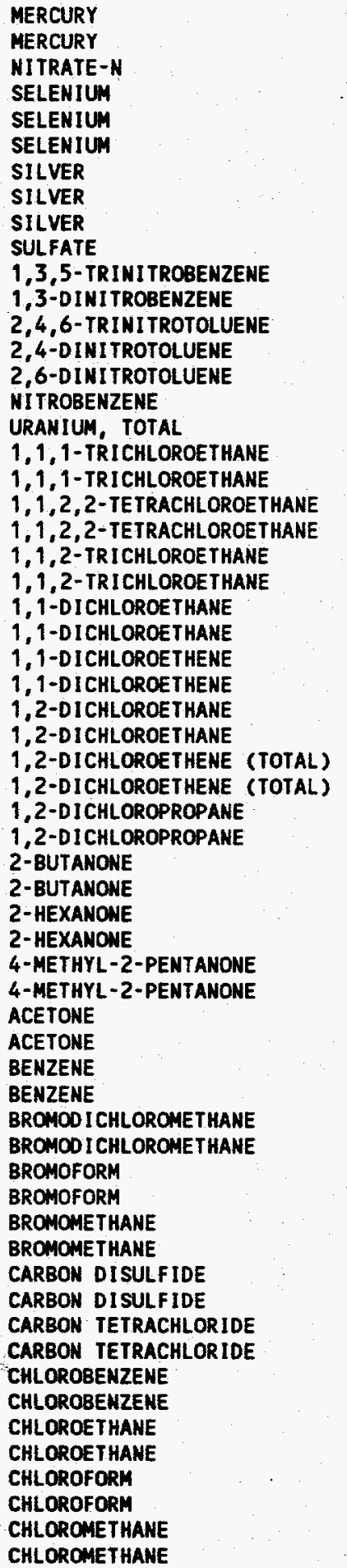 & 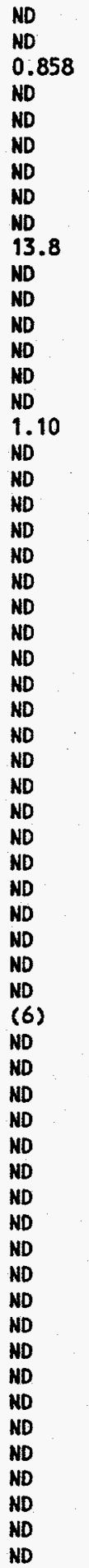 & 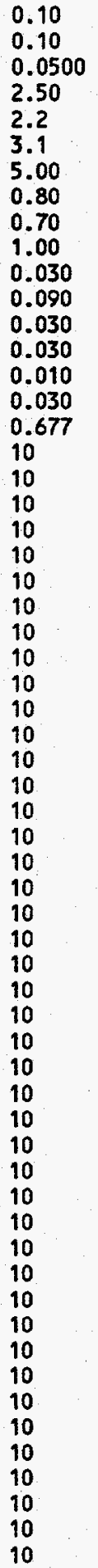 & 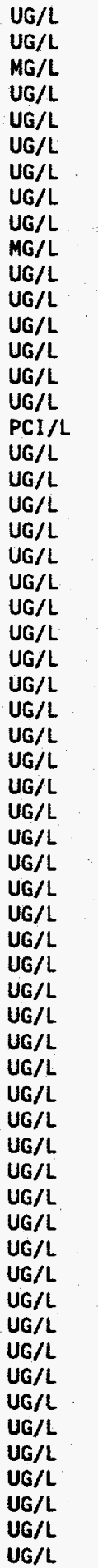 & $\begin{array}{l}\text { EPA CLP } \\
\text { EPA CLP } \\
\text { EPA } 353.2 \\
\text { EPA CLP } \\
\text { EPA CLP } \\
\text { EPA CLP } \\
\text { EPA CLP } \\
\text { EPA CLP } \\
\text { EPA CLP } \\
\text { EPA } 300.0 \\
\text { USATHAMA } \\
\text { USATHAMA } \\
\text { USATHAMA } \\
\text { USATHAMA } \\
\text { USATHAMA } \\
\text { USATHAMA } \\
\text { ASTM } 5174-91 \\
\text { EPA CLP } \\
\text { EPA CLP } \\
\text { EPA CLP } \\
\text { EPA CLP } \\
\text { EPA CLP } \\
\text { EPA CLP } \\
\text { EPA CLP } \\
\text { EPA CLP } \\
\text { EPA CLP } \\
\text { EPA CLP } \\
\text { EPA CLP } \\
\text { EPA CLP } \\
\text { EPA CLP } \\
\text { EPA CLP } \\
\text { EPA CLP } \\
\text { EPA CLP } \\
\text { EPA CLP } \\
\text { EPA CLP } \\
\text { EPA CLP } \\
\text { EPA CLP } \\
\text { EPA CLP } \\
\text { EPA CLP } \\
\text { EPA CLP } \\
\text { EPA CLP } \\
\text { EPA CLP } \\
\text { EPA CLP } \\
\text { EPA CLP } \\
\text { EPA CLP } \\
\text { EPA CLP } \\
\text { EPA CLP } \\
\text { EPA CLP } \\
\text { EPA CLP } \\
\text { EPA CLP } \\
\text { EPA CLP } \\
\text { EPA CLP } \\
\text { EPA CLP } \\
\text { EPA CLP } \\
\text { EPA CLP } \\
\text { EPA CLP } \\
\text { EPA CLP } \\
\text { EPA CLP } \\
\text { EPA CLP } \\
\text { EPA CLP } \\
\text { EPA CLP } \\
\end{array}$ \\
\hline
\end{tabular}




\begin{tabular}{|c|c|c|c|c|c|c|}
\hline WSSRAP_ID & DATE_SAM & PARAMETER & CONC & $\mathrm{DL}$ & UNITS & METHOD \\
\hline 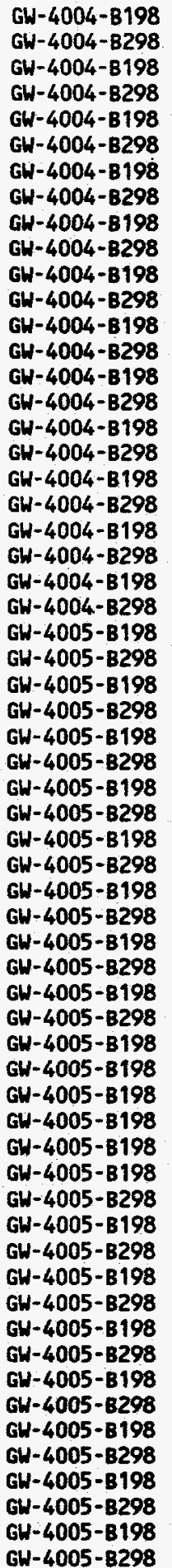 & $\begin{array}{l}01 / 29 / 98 \\
03 / 25 / 98 \\
01 / 29 / 98 \\
03 / 25 / 98 \\
01 / 29 / 98 \\
03 / 25 / 98 \\
01 / 29 / 98 \\
03 / 25 / 98 \\
01 / 29 / 98 \\
03 / 25 / 98 \\
01 / 29 / 98 \\
03 / 25 / 98 \\
01 / 29 / 98 \\
03 / 25 / 98 \\
01 / 29 / 98 \\
03 / 25 / 98 \\
01 / 29 / 98 \\
03 / 25 / 98 \\
01 / 29 / 98 \\
03 / 25 / 98 \\
01 / 29 / 98 \\
03 / 25 / 98 \\
01 / 29 / 98 \\
03 / 25 / 98 \\
02 / 02 / 98 \\
03 / 25 / 98 \\
02 / 02 / 98 \\
03 / 25 / 98 \\
02 / 02 / 98 \\
03 / 25 / 98 \\
02 / 02 / 98 \\
03 / 25 / 98 \\
02 / 02 / 98 \\
03 / 25 / 98 \\
02 / 02 / 98 \\
03 / 25 / 98 \\
02 / 02 / 98 \\
03 / 25 / 98 \\
02 / 02 / 98 \\
03 / 25 / 98 \\
02 / 02 / 98 \\
02 / 02 / 98 \\
02 / 02 / 98 \\
02 / 02 / 98 \\
02 / 02 / 98 \\
02 / 02 / 98 \\
03 / 25 / 98 \\
02 / 02 / 98 \\
03 / 25 / 98 \\
02 / 02 / 98 \\
03 / 25 / 98 \\
02 / 02 / 98 \\
03 / 25 / 98 \\
02 / 02 / 98 \\
03 / 25 / 98 \\
02 / 02 / 98 \\
03 / 25 / 98 \\
02 / 02 / 98 \\
03 / 25 / 98 \\
02 / 02 / 98 \\
03 / 25 / 98\end{array}$ & 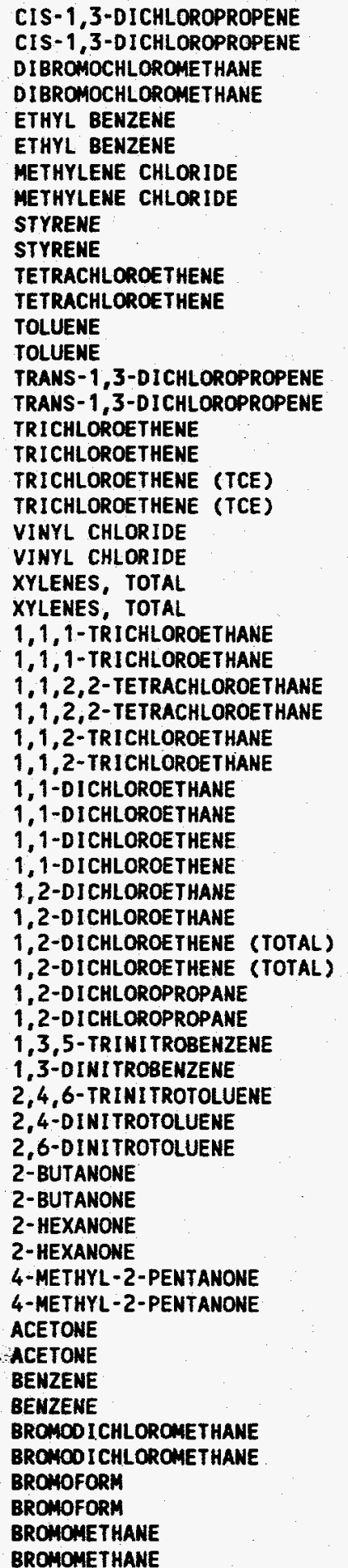 & $\begin{array}{l}\text { ND } \\
\text { ND } \\
\text { ND } \\
\text { ND } \\
\text { ND } \\
\text { ND } \\
\text { (4) } \\
\text { ND } \\
\text { ND } \\
\text { ND } \\
\text { ND } \\
\text { ND } \\
\text { ND } \\
\text { ND } \\
\text { ND } \\
\text { ND } \\
\text { ND } \\
\text { ND } \\
\text { ND } \\
\text { ND } \\
\text { ND } \\
\text { ND } \\
\text { ND } \\
\text { ND } \\
\text { ND } \\
\text { ND } \\
\text { ND } \\
\text { ND } \\
\text { ND } \\
\text { ND } \\
\text { ND } \\
\text { ND } \\
\text { ND } \\
\text { ND } \\
\text { ND } \\
\text { ND } \\
\text { ND } \\
\text { ND } \\
\text { ND } \\
\text { ND } \\
\text { ND } \\
\text { ND } \\
\text { ND } \\
\text { ND } \\
\text { ND } \\
\text { ND } \\
\text { ND } \\
\text { ND } \\
\text { ND } \\
\text { ND } \\
\text { ND } \\
\text { (2) } \\
\text { ND } \\
\text { ND } \\
\text { ND } \\
\text { ND } \\
\text { ND } \\
\text { ND } \\
\text { ND } \\
\text { ND }\end{array}$ & $\begin{array}{l}10 \\
10 \\
10 \\
10 \\
10 \\
10 \\
10 \\
10 \\
10 \\
10 \\
10 \\
10 \\
10 \\
10 \\
10 \\
10 \\
10 \\
10 \\
1 \\
1 \\
10 \\
10 \\
10 \\
10 \\
10 \\
10 \\
10 \\
10 \\
10 \\
10 \\
10 \\
10 \\
10 \\
10 \\
10 \\
10 \\
10 \\
10 \\
10 \\
10 \\
0.030 \\
0.090 \\
0.030 \\
0.030 \\
0.010 \\
10 \\
10 \\
10 \\
10 \\
10 \\
10 \\
10 \\
10 \\
10 \\
10 \\
10 \\
10 \\
10 \\
10 \\
10 \\
10 \\
\\
10 \\
\\
10 \\
\\
10 \\
\end{array}$ & $\begin{array}{l}\text { UG/L } \\
U G / L \\
U G / L \\
U G / L \\
U G / L \\
U G / L \\
U G / L \\
U G / L \\
U G / L \\
U G / L \\
U G / L \\
U G / L \\
U G / L \\
U G / L \\
U G / L \\
U G / L \\
U G / L \\
U G / L \\
U G / L \\
U G / L \\
U G / L \\
U G / L \\
U G / L \\
U G / L \\
U G / L \\
U G / L \\
U G / L \\
U G / L \\
U G / L \\
U G / L \\
U G / L \\
U G / L \\
U G / L \\
U G / L \\
U G / L \\
U G / L \\
U G / L \\
U G / L \\
U G / L \\
U G / L \\
U G / L \\
U G / L \\
U G / L \\
U G / L \\
U G / L \\
U G / L \\
U G / L \\
U G / L \\
U G / L \\
U G / L \\
U G / L \\
U G / L \\
U G / L \\
U G / L \\
U G / L \\
U G / L \\
U G / L \\
U G / L \\
U G / L \\
U G / L \\
U G / L\end{array}$ & $\begin{array}{l}\text { EPA CLP } \\
\text { EPA CLP } \\
\text { EPA CLP } \\
\text { EPA CLP } \\
\text { EPA CLP } \\
\text { EPA CLP } \\
\text { EPA CLP } \\
\text { EPA CLP } \\
\text { EPA CLP } \\
\text { EPA CLP } \\
\text { EPA CLP } \\
\text { EPA CLP } \\
\text { EPA CLP } \\
\text { EPA CLP } \\
\text { EPA CLP } \\
\text { EPA CLP } \\
\text { EPA CLP } \\
\text { EPA CLP } \\
\text { EPA } 8260 \\
\text { EPA } 8260 \\
\text { EPA CLP } \\
\text { EPA CLP } \\
\text { EPA CLP } \\
\text { EPA CLP } \\
\text { EPA CLP } \\
\text { EPA CLP } \\
\text { EPA CLP } \\
\text { EPA CLP } \\
\text { EPA CLP } \\
\text { EPA CLP } \\
\text { EPA CLP } \\
\text { EPA CLP } \\
\text { EPA CLP } \\
\text { EPA CLP } \\
\text { EPA CLP } \\
\text { EPA CLP } \\
\text { EPA CLP } \\
\text { EPA CLP } \\
\text { EPA CLP } \\
\text { EPA CLP } \\
\text { USATHAMA } \\
\text { USATHAMA } \\
\text { USATHAMA } \\
\text { USATHAMA } \\
\text { USATHAMA } \\
\text { EPA CLP } \\
\text { EPA CLP } \\
\text { EPA CLP } \\
\text { EPA CLP } \\
\text { EPA CLP } \\
\text { EPA CLP } \\
\text { EPA CLP } \\
\text { EPA CLP } \\
\text { EPA CLP } \\
\text { EPA CLP } \\
\text { EPA CLP } \\
\text { EPA CLP } \\
\text { EPA CLP } \\
\text { EPA CLP } \\
\text { EPA CLP } \\
\text { EPAP }\end{array}$ \\
\hline
\end{tabular}




\begin{tabular}{|c|c|c|c|c|c|c|}
\hline WSSRAP_ID & DATE_SAM & PARAMETER & CONC & DL & UNITS & METHOD \\
\hline $\begin{array}{l}\text { GW-4005-B198 } \\
G W-4005-8298 \\
G W-4005-8198 \\
G W-4005-B 298 \\
G W-4005-B 198 \\
G W-4005-B 298 \\
G W-4005-B 198 \\
G W-4005-8298 \\
G W-4005-B 198 \\
G W-4005-B 298 \\
G W-4005-B 198 \\
G W-4005-B 298 \\
G W-4005-8198 \\
G W-4005-B 298 \\
G W-4005-B 198 \\
G W-4005-B 298 \\
G W-4005-B 198 \\
G W-4005-8298 \\
G W-4005-B 198 \\
G W-4005-B 298 \\
G W-4005-B 198 \\
G W-4005-B 198 \\
G W-4005-B 298 \\
G W-4005-B 198 \\
G W-4005-B 298 \\
G W-4005-B 198 \\
G W-4005-B 298 \\
G W-4005-B 198 \\
G W-4005-B 298 \\
G W-4005-B 198 \\
G W-4005-B 298 \\
G W-4005-B 198 \\
G W-4005-B 298 \\
G W-4005-B 198 \\
G W-4005-8298 \\
G W-4005-B 198 \\
G W-4005-B 298 \\
G W-4006-0997 \\
G W-4006-B 198 \\
G W-4006-8298 \\
G W-4006-0997 \\
G W-4006-B 198 \\
G W-4006-B 298 \\
G W-4006-0997 \\
G W-4006-8198 \\
G W-4006-B 298 \\
G W-4006-0997 \\
G W-4006-8198 \\
G W-4006-B 298 \\
G W-4006-0997 \\
G W-4006-B 198 \\
G W-4006-B 298 \\
G W-4006-0997 \\
G W-4006-8198 \\
G W-4006-8298 \\
G W-4006-09997 \\
G W-4006-0997 \\
G W-4006-8198 \\
G W-4006-8298 \\
G W-4006-09997 \\
G W-4006-B 198\end{array}$ & $\begin{array}{l}02 / 02 / 98 \\
03 / 25 / 98 \\
02 / 02 / 98 \\
03 / 25 / 98 \\
02 / 02 / 98 \\
03 / 25 / 98 \\
02 / 02 / 98 \\
03 / 25 / 98 \\
02 / 02 / 98 \\
03 / 25 / 98 \\
02 / 02 / 98 \\
03 / 25 / 98 \\
02 / 02 / 98 \\
03 / 25 / 98 \\
02 / 02 / 98 \\
03 / 25 / 98 \\
02 / 02 / 98 \\
03 / 25 / 98 \\
02 / 02 / 98 \\
03 / 25 / 98 \\
02 / 02 / 98 \\
02 / 02 / 98 \\
03 / 25 / 98 \\
02 / 02 / 98 \\
03 / 25 / 98 \\
02 / 02 / 98 \\
03 / 25 / 98 \\
02 / 02 / 98 \\
03 / 25 / 98 \\
02 / 02 / 98 \\
03 / 25 / 98 \\
02 / 02 / 98 \\
03 / 25 / 98 \\
02 / 02 / 98 \\
03 / 25 / 98 \\
02 / 02 / 98 \\
03 / 25 / 98 \\
09 / 23 / 97 \\
02 / 03 / 98 \\
03 / 27 / 98 \\
09 / 23 / 97 \\
02 / 03 / 98 \\
03 / 27 / 98 \\
09 / 23 / 97 \\
02 / 03 / 98 \\
03 / 27 / 98 \\
09 / 23 / 97 \\
02 / 03 / 98 \\
03 / 27 / 98 \\
09 / 23 / 97 \\
02 / 03 / 98 \\
03 / 27 / 98 \\
09 / 23 / 97 \\
02 / 03 / 98 \\
03 / 27 / 98 \\
09 / 23 / 97 \\
09 / 23 / 97 \\
02 / 03 / 98 \\
03 / 27 / 98 \\
09 / 23 / 97 \\
02 / 03 / 98\end{array}$ & 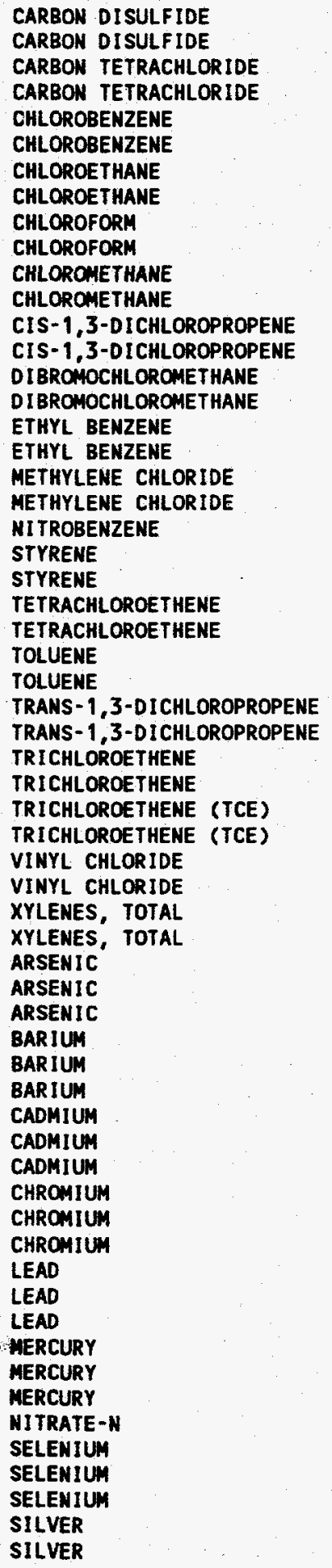 & $\begin{array}{l}\text { ND } \\
\text { ND } \\
\text { ND } \\
\text { ND } \\
\text { ND } \\
\text { ND } \\
\text { ND } \\
\text { ND } \\
\text { ND } \\
\text { ND } \\
\text { ND } \\
\text { ND } \\
\text { ND } \\
\text { ND } \\
\text { ND } \\
\text { ND } \\
\text { ND } \\
\text { (2) } \\
\text { (3) } \\
\text { ND } \\
\text { ND } \\
\text { ND } \\
\text { ND } \\
\text { ND } \\
\text { ND } \\
\text { ND } \\
\text { ND } \\
\text { ND } \\
\text { ND } \\
\text { ND } \\
\text { ND } \\
\text { ND } \\
\text { ND } \\
\text { ND } \\
\text { ND } \\
\text { ND } \\
\text { ND } \\
\text { ND } \\
\text { ND } \\
215 \\
222 \\
233 \\
\text { ND } \\
\text { ND } \\
\text { ND } \\
\text { ND } \\
\text { ND } \\
8.5 \\
5.10 \\
\text { ND } \\
\text { ND.4 } \\
\text { ND } \\
\text { ND } \\
\text { ND } \\
\text { ND } \\
\text { ND } \\
\text { ND } \\
\text { ND } \\
\text { ND }\end{array}$ & $\begin{array}{l}10 \\
10 \\
10 \\
10 \\
10 \\
10 \\
10 \\
10 \\
10 \\
10 \\
10 \\
10 \\
10 \\
10 \\
10 \\
10 \\
10 \\
10 \\
10 \\
10 \\
0.030 \\
10 \\
10 \\
10 \\
10 \\
10 \\
10 \\
10 \\
10 \\
10 \\
10 \\
5 \\
1 \\
10 \\
10 \\
10 \\
10 \\
2.00 \\
1.3 \\
1.9 \\
5.00 \\
0.40 \\
1.0 \\
5.00 \\
0.40 \\
0.60 \\
10.0 \\
4.70 \\
0.80 \\
1.50 \\
6.40 \\
1.1 \\
0.100 \\
0.10 \\
0.10 \\
2.50 \\
2.50 \\
2.2 \\
3.1 \\
5.00 \\
0.80 \\
\end{array}$ & $\begin{array}{l}U G / L \\
U G / L \\
U G / L \\
U G / L \\
U G / L \\
U G / L \\
U G / L \\
U G / L \\
U G / L \\
U G / L \\
U G / L \\
U G / L \\
U G / L \\
U G / L \\
U G / L \\
U G / L \\
U G / L \\
U G / L \\
U G / L \\
U G / L \\
U G / L \\
U G / L \\
U G / L \\
U G / L \\
U G / L \\
U G / L \\
U G / L \\
U G / L \\
U G / L \\
U G / L \\
U G / L \\
U G / L \\
U G / L \\
U G / L \\
U G / L \\
U G / L \\
U G / L \\
U G / L \\
U G / L \\
U G / L \\
U G / L \\
U G / L \\
U G / L \\
U G / L \\
U G / L \\
U G / L \\
U G / L \\
U G / L \\
U G / L \\
U G / L \\
U G / L \\
U G / L \\
U G / L \\
U G / L \\
U G / L \\
U G / L \\
U G / L \\
U G / L \\
U G / L \\
U G / L \\
U G / L \\
U\end{array}$ & $\begin{array}{l}\text { EPA CLP } \\
\text { EPA CLP } \\
\text { EPA CLP } \\
\text { EPA CLP } \\
\text { EPA CLP } \\
\text { EPA CLP } \\
\text { EPA CLP } \\
\text { EPA CLP } \\
\text { EPA CLP } \\
\text { EPA CLP } \\
\text { EPA CLP } \\
\text { EPA CLP } \\
\text { EPA CLP } \\
\text { EPA CLP } \\
\text { EPA CLP } \\
\text { EPA CLP } \\
\text { EPA CLP } \\
\text { EPA CLP } \\
\text { EPA CLP } \\
\text { EPA CLP } \\
\text { USATHAMA } \\
\text { EPA CLP } \\
\text { EPA CLP } \\
\text { EPA CLP } \\
\text { EPA CLP } \\
\text { EPA CLP } \\
\text { EPA CLP } \\
\text { EPA CLP } \\
\text { EPA CLP } \\
\text { EPA CLP } \\
\text { EPA CLP } \\
\text { EPA } 8260 \\
\text { EPA } 8260 \\
\text { EPA CLP } \\
\text { EPA CLP } \\
\text { EPA CLP } \\
\text { EPA CLP } \\
\text { EPA CLP } \\
\text { EPA CLP } \\
\text { EPA CLP } \\
\text { EPA CLP } \\
\text { EPA CLP } \\
\text { EPA CLP } \\
\text { EPA CLP } \\
\text { EPA CLP } \\
\text { EPA CLP } \\
\text { EPA CLP } \\
\text { EPA CLP } \\
\text { EPA CLP } \\
\text { EPA CLP } \\
\text { EPA CLP } \\
\text { EPA CLP } \\
\text { EPA CLP } \\
\text { EPA CLP } \\
\text { EPA CLP } \\
\text { EPA } 353.2 \\
\text { EPA CLP } \\
\text { EPA CLP } \\
\text { EPA CLP } \\
\text { EPA CLP } \\
\text { EPA CLP }\end{array}$ \\
\hline
\end{tabular}




\begin{tabular}{|c|c|c|c|c|c|c|}
\hline WSSRAP_ID & DATE_SAM & PARAMETER & CONC & $D L$ & UNITS & METHOD \\
\hline 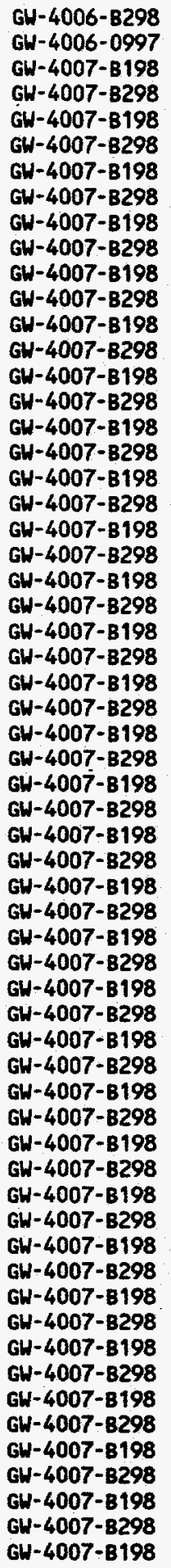 & $\begin{array}{l}03 / 27 / 98 \\
09 / 23 / 97 \\
02 / 02 / 98 \\
03 / 26 / 98 \\
02 / 02 / 98 \\
03 / 26 / 98 \\
02 / 02 / 98 \\
03 / 26 / 98 \\
02 / 02 / 98 \\
03 / 26 / 98 \\
02 / 02 / 98 \\
03 / 26 / 98 \\
02 / 02 / 98 \\
03 / 26 / 98 \\
02 / 02 / 98 \\
03 / 26 / 98 \\
02 / 02 / 98 \\
03 / 26 / 98 \\
02 / 02 / 98 \\
03 / 26 / 98 \\
02 / 02 / 98 \\
03 / 26 / 98 \\
02 / 02 / 98 \\
03 / 26 / 98 \\
02 / 02 / 98 \\
03 / 26 / 98 \\
02 / 02 / 98 \\
03 / 26 / 98 \\
02 / 02 / 98 \\
03 / 26 / 98 \\
02 / 02 / 98 \\
03 / 26 / 98 \\
02 / 02 / 98 \\
03 / 26 / 98 \\
02 / 02 / 98 \\
03 / 26 / 98 \\
02 / 02 / 98 \\
03 / 26 / 98 \\
02 / 02 / 98 \\
03 / 26 / 98 \\
02 / 02 / 98 \\
03 / 26 / 98 \\
02 / 02 / 98 \\
03 / 26 / 98 \\
02 / 02 / 98 \\
03 / 26 / 98 \\
02 / 02 / 98 \\
03 / 26 / 98 \\
02 / 02 / 98 \\
03 / 26 / 98 \\
02 / 02 / 98 \\
03 / 26 / 98 \\
02 / 02 / 98 \\
03 / 26 / 98 \\
02 / 02 / 98 \\
03 / 26 / 98 \\
02 / 02 / 98 \\
03 / 26 / 98 \\
02 / 02 / 98 \\
03 / 26 / 98 \\
02 / 02 / 98\end{array}$ & 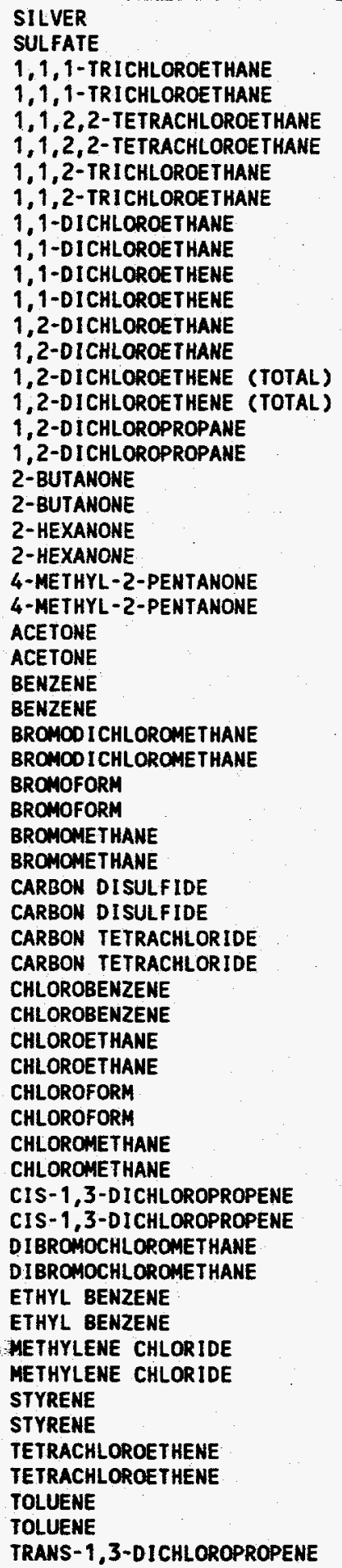 & 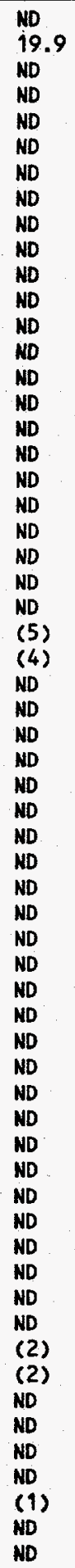 & $\begin{array}{l}0 \\
1.70 \\
10 \\
10 \\
10 \\
10 \\
10 \\
10 \\
10 \\
10 \\
10 \\
10 \\
10 \\
10 \\
10 \\
10 \\
10 \\
10 \\
10 \\
10 \\
10 \\
10 \\
10 \\
10 \\
10 \\
10 \\
10 \\
10 \\
10 \\
10 \\
10 \\
10 \\
10 \\
10 \\
10 \\
10 \\
10 \\
10 \\
10\end{array}$ & $\begin{array}{l}\text { UG/L } \\
\text { MG/L } \\
U G / L \\
U G / L \\
U G / L \\
U G / L \\
U G / L \\
U G / L \\
U G / L \\
U G / L \\
U G / L \\
U G / L \\
U G / L \\
U G / L \\
U G / L \\
U G / L \\
U G / L \\
U G / L \\
U G / L \\
U G / L \\
U G / L \\
U G / L \\
U G / L \\
U G / L \\
U G / L \\
U G / L \\
U G / L \\
U G / L \\
U G / L \\
U G / L \\
U G / L \\
U G / L \\
U G / L \\
U G / L \\
U G / L \\
U G / L \\
U G / L \\
U G / L \\
U G / L \\
U G / L \\
U G / L \\
U G / L \\
U G / L \\
U G / L \\
U G / L \\
U G / L \\
U G / L \\
U G / L \\
U G / L \\
U G / L \\
U G / L \\
U G / L \\
U G / L \\
U G / L \\
U G / L \\
U G / L \\
U G / L \\
U G / L \\
U G / L \\
U\end{array}$ & $\begin{array}{l}\text { EPA CLP } \\
\text { EPA } 300.0 \\
\text { EPA CLP } \\
\text { EPA CLP } \\
\text { EPA CLP } \\
\text { EPA CLP } \\
\text { EPA CLP } \\
\text { EPA CLP } \\
\text { EPA CLP } \\
\text { EPA CLP } \\
\text { EPA CLP } \\
\text { EPA CLP } \\
\text { EPA CLP } \\
\text { EPA CLP } \\
\text { EPA CLP } \\
\text { EPA CLP } \\
\text { EPA CLP } \\
\text { EPA CLP } \\
\text { EPA CLP } \\
\text { EPA CLP } \\
\text { EPA CLP } \\
\text { EPA CLP } \\
\text { EPA CLP } \\
\text { EPA CLP } \\
\text { EPA CLP } \\
\text { EPA CLP } \\
\text { EPA CLP } \\
\text { EPA CLP } \\
\text { EPA CLP } \\
\text { EPA CLP } \\
\text { EPA CLP } \\
\text { EPA CLP } \\
\text { EPA CLP } \\
\text { EPA CLP } \\
\text { EPA CLP } \\
\text { EPA CLP } \\
\text { EPA CLP } \\
\text { EPA CLP } \\
\text { EPA CLP } \\
\text { EPA CLP } \\
\text { EPA CLP } \\
\text { EPA CLP } \\
\text { EPA CLP } \\
\text { EPA CLP } \\
\text { EPA CLP } \\
\text { EPA CLP } \\
\text { EPA CLP } \\
\text { EPA CLP } \\
\text { EPA CLP } \\
\text { EPA CLP } \\
\text { EPA CLP } \\
\text { EPA CLP } \\
\text { EPA CLP } \\
\text { EPA CLP } \\
\text { EPA CLP } \\
\text { EPA CLP } \\
\text { EPA CLPP } \\
\text { EPA CLP } \\
\text { EPA CLP } \\
\text { EPA CLP } \\
\text { EPA CLP }\end{array}$ \\
\hline
\end{tabular}




\begin{tabular}{|c|c|c|c|c|c|c|}
\hline WSSRAP_ID & DATE_SAM & PARAMETER & CONC & DL & UNITS & METHOD \\
\hline 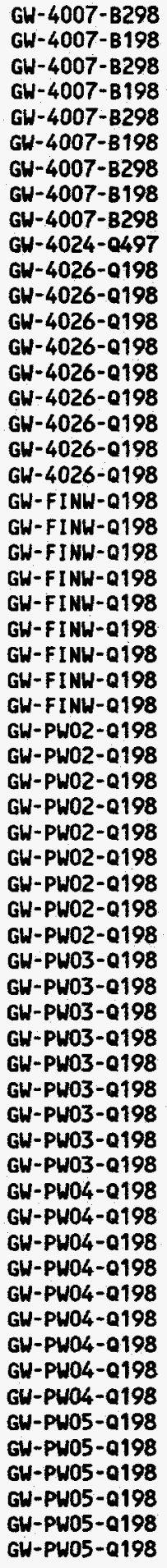 & $\begin{array}{l}03 / 26 / 98 \\
02 / 02 / 98 \\
03 / 26 / 98 \\
02 / 02 / 98 \\
03 / 26 / 98 \\
02 / 02 / 98 \\
03 / 26 / 98 \\
02 / 02 / 98 \\
03 / 26 / 98 \\
11 / 19 / 97 \\
03 / 18 / 98 \\
03 / 18 / 98 \\
03 / 18 / 98 \\
03 / 18 / 98 \\
03 / 18 / 98 \\
03 / 18 / 98 \\
03 / 18 / 98 \\
03 / 18 / 98 \\
03 / 18 / 98 \\
03 / 24 / 98 \\
03 / 24 / 98 \\
03 / 24 / 98 \\
03 / 24 / 98 \\
03 / 24 / 98 \\
03 / 24 / 98 \\
03 / 24 / 98 \\
03 / 24 / 98 \\
03 / 24 / 98 \\
03 / 24 / 98 \\
03 / 24 / 98 \\
03 / 24 / 98 \\
03 / 24 / 98 \\
03 / 24 / 98 \\
03 / 24 / 98 \\
03 / 24 / 98 \\
03 / 24 / 98 \\
03 / 24 / 98 \\
03 / 24 / 98 \\
03 / 24 / 98 \\
03 / 24 / 98 \\
03 / 24 / 98 \\
03 / 24 / 98 \\
03 / 24 / 98 \\
03 / 24 / 98 \\
03 / 24 / 98 \\
03 / 24 / 98 \\
03 / 24 / 98 \\
03 / 24 / 98 \\
03 / 24 / 98 \\
03 / 24 / 98 \\
03 / 24 / 98 \\
03 / 24 / 98 \\
03 / 24 / 98 \\
03 / 24 / 98 \\
03 / 24 / 98 \\
03 / 24 / 98 \\
03 / 24 / 98 \\
03 / 24 / 98 \\
03 / 24 / 98 \\
03 / 24 / 98 \\
03 / 24 / 98\end{array}$ & 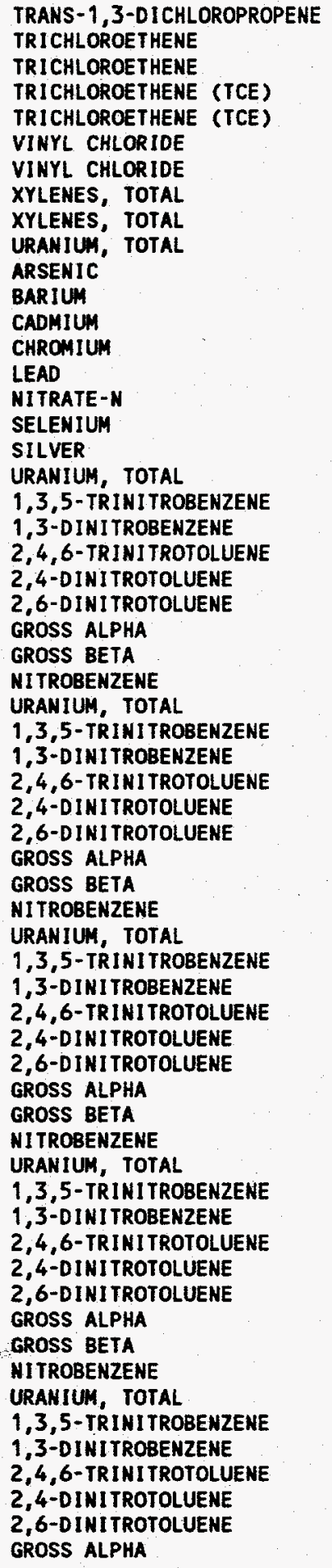 & $\begin{array}{l}\text { ND } \\
\text { ND } \\
\text { ND } \\
\text { ND } \\
\text { ND } \\
\text { ND } \\
\text { ND } \\
\text { ND } \\
6.16 \\
\text { ND } \\
1130 \\
\text { ND } \\
\text { ND } \\
\text { ND } \\
\text { O.095 } \\
\text { ND } \\
\text { ND } \\
\text { ND } \\
\text { ND } \\
\text { ND } \\
\text { ND } \\
\text { ND } \\
\text { ND } \\
3.07 \\
6.20 \\
\text { ND } \\
\text { ND } \\
\text { ND } \\
\text { ND } \\
\text { ND } \\
\text { ND } \\
\text { ND } \\
3.91 \\
7.52 \\
\text { ND } \\
\text { ND } \\
\text { ND } \\
\text { ND } \\
\text { ND } \\
\text { ND } \\
\text { ND } \\
3.65 \\
6.37 \\
\text { ND } \\
\text { ND } \\
\text { ND } \\
\text { ND } \\
\text { ND } \\
\text { ND } \\
\text { ND } \\
3.08 \\
7.08 \\
\text { ND } \\
\text { ND } \\
\text { HD } \\
\text { ND } \\
\text { ND } \\
\text { ND } \\
\text { ND } \\
3.23 \\
\end{array}$ & $\begin{array}{l}10 \\
10 \\
10 \\
5 \\
1 \\
10 \\
10 \\
10 \\
10 \\
10 \\
10 \\
0.0732 \\
1.9 \\
1.0 \\
0 \mid\end{array}$ & $\begin{array}{l}U G / L \\
U G / L \\
U G / L \\
U G / L \\
U G / L \\
U G / L \\
U G / L \\
U G / L \\
U G / L \\
P C I / L \\
U G / L \\
U G / L \\
U G / L \\
U G / L \\
U G / L \\
M G / L \\
U G / L \\
U G / L \\
P C I / L \\
U G / L \\
U G / L \\
U G / L \\
U G / L \\
U G / L \\
P C I / L \\
P C I / L \\
U G / L \\
P C I / L \\
U G / L \\
U G / L \\
U G / L \\
U G / L \\
U G / L \\
P C I / L \\
P C I / L \\
U G / L \\
P C I / L \\
U G / L \\
U G / L \\
U G / L \\
U G / L \\
U G / L \\
P C I / L \\
P C I / L \\
U G / L \\
P C I / L \\
U G / L \\
U G / L \\
U G / L \\
U G / L \\
U G / L \\
P C I / L \\
P C I / L \\
U G / L \\
P C I / L \\
U G / L \\
U G / L \\
U G / L \\
U G / L \\
U G / L \\
P C I / L \\
\text { US }\end{array}$ & $\begin{array}{l}\text { EPA CLP } \\
\text { EPA CLP } \\
\text { EPA CLP } \\
\text { EPA } 8260 \\
\text { EPA } 8260 \\
\text { EPA CLP } \\
\text { EPA CLP } \\
\text { EPA CLP } \\
\text { EPA CLP } \\
\text { EPA 6020 } \\
\text { EPA CLP } \\
\text { EPA CLP } \\
\text { EPA CLP } \\
\text { EPA CLP } \\
\text { EPA CLP } \\
\text { EPA } 353.1 \\
\text { EPA CLP } \\
\text { EPA CLP } \\
\text { ASTM } 5174-91 \\
\text { USATHAMA } \\
\text { USATHAMA } \\
\text { USATHAMA } \\
\text { USATHAMA } \\
\text { USATHAMA } \\
\text { EPA 900.0 } \\
\text { EPA 900.0 } \\
\text { USATHAMA } \\
\text { ASTM } 5174-91 \\
\text { USATHAMA } \\
\text { USATHAMA } \\
\text { USATHAMA } \\
\text { USATHAMA } \\
\text { USATHAMA } \\
\text { EPA 900.0 } \\
\text { EPA 900.0 } \\
\text { USATHAMA } \\
\text { ASTM } 5174-91 \\
\text { USATHAMA } \\
\text { USATHAMA } \\
\text { USATHAMA } \\
\text { USATHAMA } \\
\text { USATHAMA } \\
\text { EPA 900.0 } \\
\text { EPA 900.0 } \\
\text { USATHAMA } \\
\text { ASTM } 5174-91 \\
\text { USATHAMA } \\
\text { USATHAMA } \\
\text { USATHAMA } \\
\text { USATHAMA } \\
\text { USATHAMA } \\
\text { EPA 900.0 } \\
\text { EPA } 900.0 \\
\text { USATHAMA } \\
\text { ASTM } 5174-91 \\
\text { USATHAMA } \\
\text { USATHAMA } \\
\text { USATHAMA } \\
\text { USATHAMA } \\
\text { USATHAMA } \\
\text { EPA 900.0 }\end{array}$ \\
\hline
\end{tabular}




\begin{tabular}{|c|c|c|c|c|c|c|}
\hline WSSRAP_ID & DATE_SAM & PARAMETER & CONC & DL & UNITS & METHOD \\
\hline 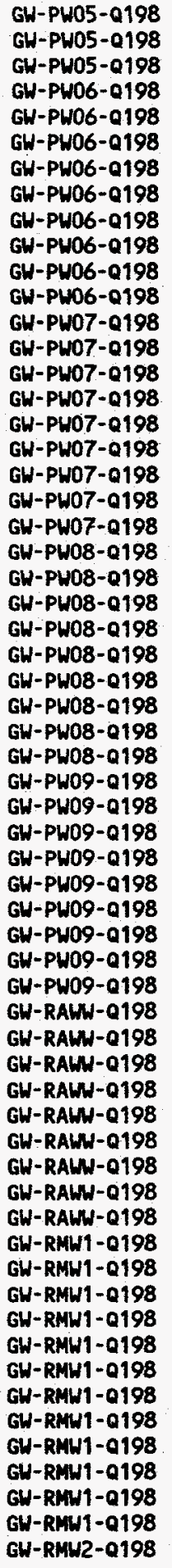 & $\begin{array}{l}03 / 24 / 98 \\
03 / 24 / 98 \\
03 / 24 / 98 \\
03 / 24 / 98 \\
03 / 24 / 98 \\
03 / 24 / 98 \\
03 / 24 / 98 \\
03 / 24 / 98 \\
03 / 24 / 98 \\
03 / 24 / 98 \\
03 / 24 / 98 \\
03 / 24 / 98 \\
03 / 24 / 98 \\
03 / 24 / 98 \\
03 / 24 / 98 \\
03 / 24 / 98 \\
03 / 24 / 98 \\
03 / 24 / 98 \\
03 / 24 / 98 \\
03 / 24 / 98 \\
03 / 24 / 98 \\
03 / 24 / 98 \\
03 / 24 / 98 \\
03 / 24 / 98 \\
03 / 24 / 98 \\
03 / 24 / 98 \\
03 / 24 / 98 \\
03 / 24 / 98 \\
03 / 24 / 98 \\
03 / 24 / 98 \\
03 / 24 / 98 \\
03 / 24 / 98 \\
03 / 24 / 98 \\
03 / 24 / 98 \\
03 / 24 / 98 \\
03 / 24 / 98 \\
03 / 24 / 98 \\
03 / 24 / 98 \\
03 / 24 / 98 \\
03 / 24 / 98 \\
03 / 24 / 98 \\
03 / 24 / 98 \\
03 / 24 / 98 \\
03 / 24 / 98 \\
03 / 24 / 98 \\
03 / 24 / 98 \\
03 / 24 / 98 \\
03 / 24 / 98 \\
03 / 24 / 98 \\
03 / 24 / 98 \\
03 / 24 / 98 \\
03 / 24 / 98 \\
03 / 24 / 98 \\
03 / 24 / 98 \\
03 / 24 / 98 \\
03 / 24 / 98 \\
03 / 24 / 98 \\
03 / 24 / 98 \\
03 / 24 / 98 \\
03 / 24 / 98 \\
03 / 27 / 98\end{array}$ & 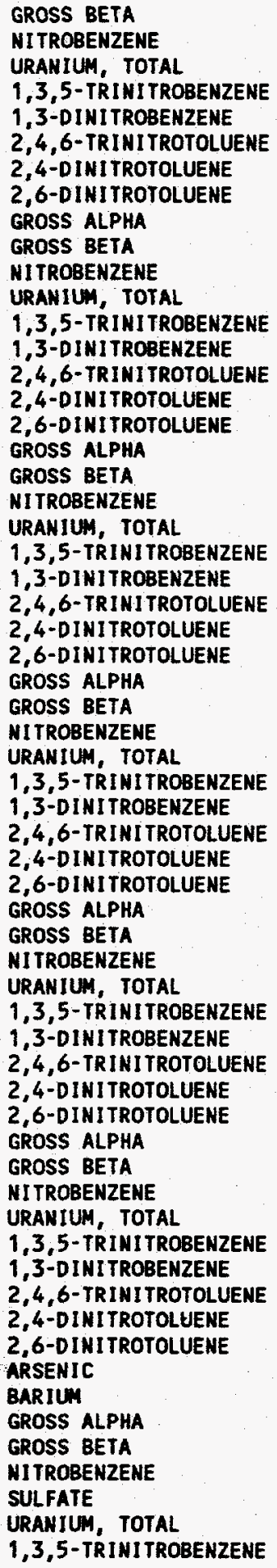 & $\begin{array}{l}6.25 \\
\text { ND } \\
\text { ND } \\
\text { ND } \\
\text { ND } \\
\text { ND } \\
\text { ND } \\
3.70 \\
7.05 \\
\text { ND } \\
\text { ND } \\
\text { ND } \\
\text { ND } \\
\text { ND } \\
\text { ND } \\
\text { ND } \\
3.77 \\
6.04 \\
\text { ND } \\
\text { ND } \\
\text { ND } \\
\text { ND } \\
\text { ND } \\
\text { ND } \\
\text { ND } \\
4.20 \\
4.92 \\
\text { ND } \\
\text { ND } \\
\text { ND } \\
\text { ND } \\
\text { ND } \\
\text { ND } \\
\text { ND } \\
4.78 \\
4.91 \\
\text { ND } \\
\text { ND } \\
\text { ND } \\
\text { ND } \\
\text { ND } \\
\text { ND } \\
\text { ND } \\
3.56 \\
5.70 \\
\text { ND } \\
\text { ND } \\
\text { ND } \\
\text { ND } \\
\text { ND } \\
\text { ND } \\
\text { ND } \\
5.7 \\
\text { NA1 } \\
3.96 \\
\text { ND } \\
\text { ND } \\
\text { ND } \\
\text { ND }\end{array}$ & $\begin{array}{l}0.810 \\
0.030 \\
0.677 \\
0.030 \\
0.090 \\
0.030 \\
0.030 \\
0.010 \\
0.979 \\
0.805 \\
0.030 \\
0.677 \\
0.030 \\
0.090 \\
0.030 \\
0.030 \\
0.010 \\
0.649 \\
0.837 \\
0.030 \\
0.677 \\
0.030 \\
0.090 \\
0.030 \\
0.030 \\
0.010 \\
0.898 \\
0.766 \\
0.030 \\
0.677 \\
0.030 \\
0.090 \\
0.030 \\
0.030 \\
0.010 \\
0.949 \\
0.763 \\
0.030 \\
0.677 \\
0.030 \\
0.090 \\
0.030 \\
0.030 \\
0.010 \\
1.12 \\
0.791 \\
0.030 \\
0.677 \\
0.030 \\
0.090 \\
0.030 \\
0.030 \\
0.010 \\
1.9 \\
1.0 \\
0.990 \\
0.742 \\
0.030 \\
1.00 \\
0.677 \\
0.030\end{array}$ & $\begin{array}{l}P C I / L \\
U G / L \\
P C I / L \\
U G / L \\
U G / L \\
U G / L \\
U G / L \\
U G / L \\
P C I / L \\
P C I / L \\
U G / L \\
P C I / L \\
U G / L \\
U G / L \\
U G / L \\
U G / L \\
U G / L \\
P C I / L \\
P C I / L \\
U G / L \\
P C I / L \\
U G / L \\
U G / L \\
U G / L \\
U G / L \\
U G / L \\
P C I / L \\
P C I / L \\
U G / L \\
P C I / L \\
U G / L \\
U G / L \\
U G / L \\
U G / L \\
U G / L \\
P C I / L \\
P C I / L \\
U G / L \\
P C I / L \\
U G / L \\
U G / L \\
U G / L \\
U G / L \\
U G / L \\
P C I / L \\
P C I / L \\
U G / L \\
P C I / L \\
U G / L \\
U G / L \\
U G / L \\
U G / L \\
U G / L \\
U G / L \\
U G / L \\
P C I / L \\
P C I / L \\
U G / L \\
U G / L \\
P C I / L \\
U G / L \\
\end{array}$ & $\begin{array}{l}\text { EPA } 900.0 \\
\text { USATHAMA } \\
\text { ASTM } 5174-91 \\
\text { USATHAMA } \\
\text { USATHAMA } \\
\text { USATHAMA } \\
\text { USATHAMA } \\
\text { USATHAMA } \\
\text { EPA } 900.0 \\
\text { EPA } 900.0 \\
\text { USATHAMA } \\
\text { ASTM } 5174-91 \\
\text { USATHAMA } \\
\text { USATHAMA } \\
\text { USATHAMA } \\
\text { USATHAMA } \\
\text { USATHAMA } \\
\text { EPA } 900.0 \\
\text { EPA } 900.0 \\
\text { USATHAMA } \\
\text { ASTM } 5174-91 \\
\text { USATHAMA } \\
\text { USATHAMA } \\
\text { USATHAMA } \\
\text { USATHAMA } \\
\text { USATHAMA } \\
\text { EPA 900.0 } \\
\text { EPA } 900.0 \\
\text { USATHAMA } \\
\text { ASTM } 5174-91 \\
\text { USATHAMA } \\
\text { USATHAMA } \\
\text { USATHAMA } \\
\text { USATHAMA } \\
\text { USATHAMA } \\
\text { EPA 900.0 } \\
\text { EPA } 900.0 \\
\text { USATHAMA } \\
\text { ASTM } 5174-91 \\
\text { USATHAMA } \\
\text { USATHAMA } \\
\text { USATHAMA } \\
\text { USATHAMA } \\
\text { USATHAMA } \\
\text { EPA 900.0 } \\
\text { EPA 900.0 } \\
\text { USATHAMA } \\
\text { ASTM } 5174-91 \\
\text { USATHAMA } \\
\text { USATHAMA } \\
\text { USATHAMA } \\
\text { USATHAMA } \\
\text { USATHAMA } \\
\text { EPA CLP } \\
\text { EPA CLP } \\
\text { EPA } 900.0 \\
\text { EPA } 900.0 \\
\text { USATHAMA } \\
\text { EPA } 300.0 \\
\text { ASTM } 5174-91 \\
\text { USATHAMA } \\
\end{array}$ \\
\hline
\end{tabular}




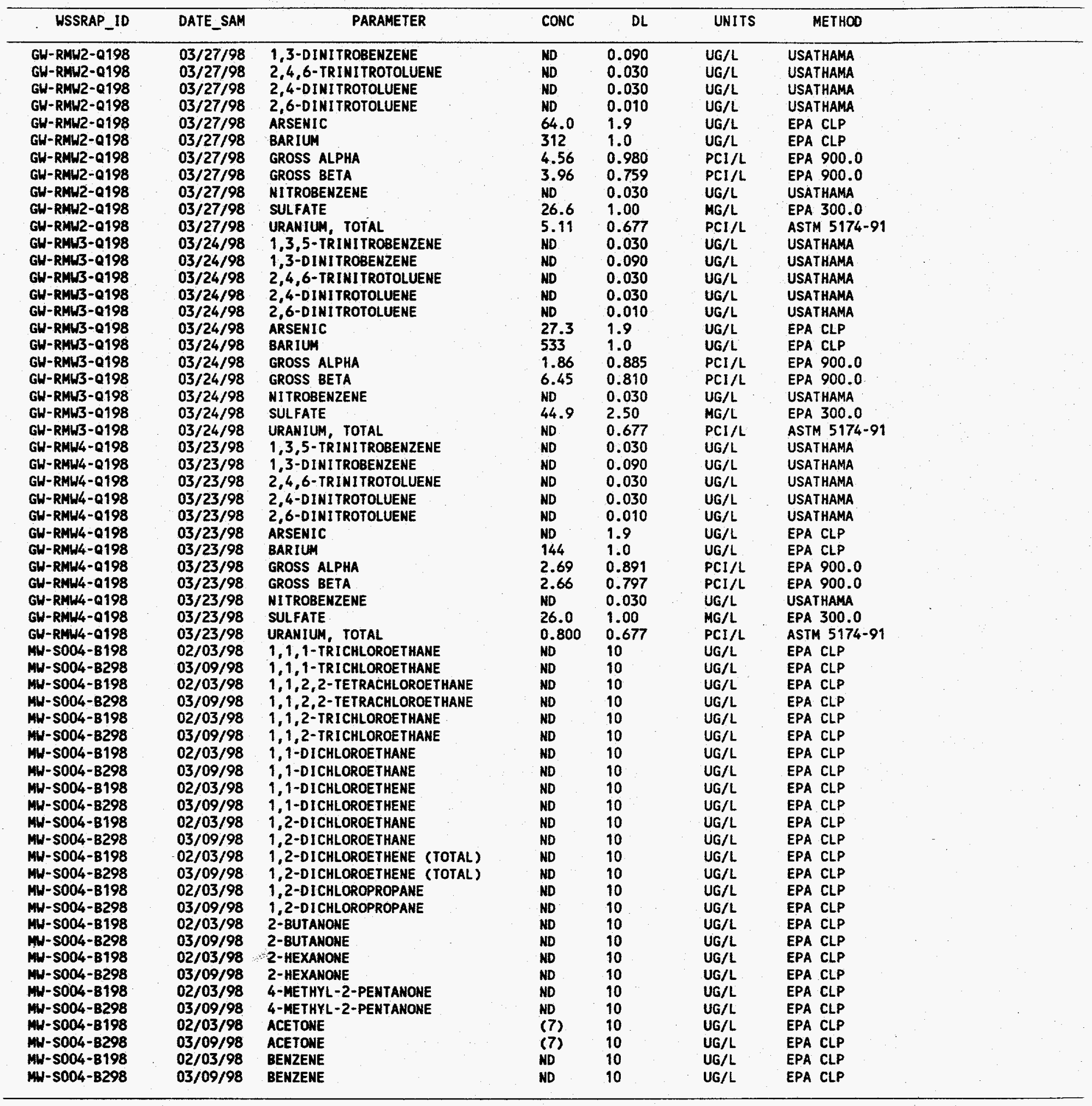




\begin{tabular}{|c|c|c|c|c|c|c|}
\hline USSRAP_ID & DATE_SAM & PARAMETER & CONC & $\mathrm{DL}$ & UNITS & METHOD \\
\hline 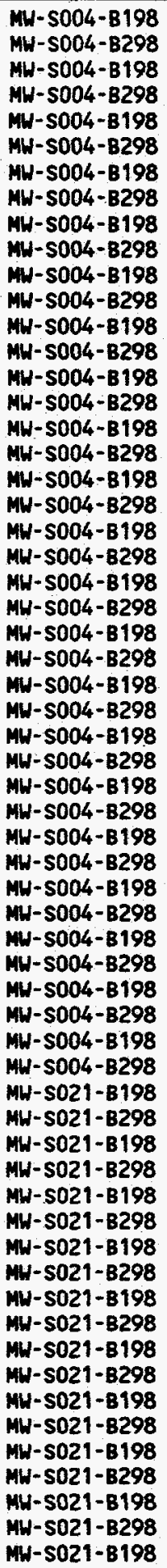 & $\begin{array}{l}02 / 03 / 98 \\
03 / 09 / 98 \\
02 / 03 / 98 \\
03 / 09 / 98 \\
02 / 03 / 98 \\
03 / 09 / 98 \\
02 / 03 / 98 \\
03 / 09 / 98 \\
02 / 03 / 98 \\
03 / 09 / 98 \\
02 / 03 / 98 \\
03 / 09 / 98 \\
02 / 03 / 98 \\
03 / 09 / 98 \\
02 / 03 / 98 \\
03 / 09 / 98 \\
02 / 03 / 98 \\
03 / 09 / 98 \\
02 / 03 / 98 \\
03 / 09 / 98 \\
02 / 03 / 98 \\
03 / 09 / 98 \\
02 / 03 / 98 \\
03 / 09 / 98 \\
02 / 03 / 98 \\
03 / 09 / 98 \\
02 / 03 / 98 \\
03 / 09 / 98 \\
02 / 03 / 98 \\
03 / 09 / 98 \\
02 / 03 / 98 \\
03 / 09 / 98 \\
02 / 03 / 98 \\
03 / 09 / 98 \\
02 / 03 / 98 \\
03 / 09 / 98 \\
02 / 03 / 98 \\
03 / 09 / 98 \\
02 / 03 / 98 \\
03 / 09 / 98 \\
02 / 03 / 98 \\
03 / 09 / 98 \\
02 / 03 / 98 \\
03 / 10 / 98 \\
02 / 03 / 98 \\
03 / 10 / 98 \\
02 / 03 / 98 \\
03 / 10 / 98 \\
02 / 03 / 98 \\
03 / 10 / 98 \\
02 / 03 / 98 \\
03 / 10 / 98 \\
02 / 03 / 98 \\
03 / 10 / 98 \\
02 / 03 / 98 \\
03 / 10 / 98 \\
02 / 03 / 98 \\
03 / 10 / 98 \\
02 / 03 / 98 \\
03 / 10 / 98 \\
02 / 03 / 98\end{array}$ & 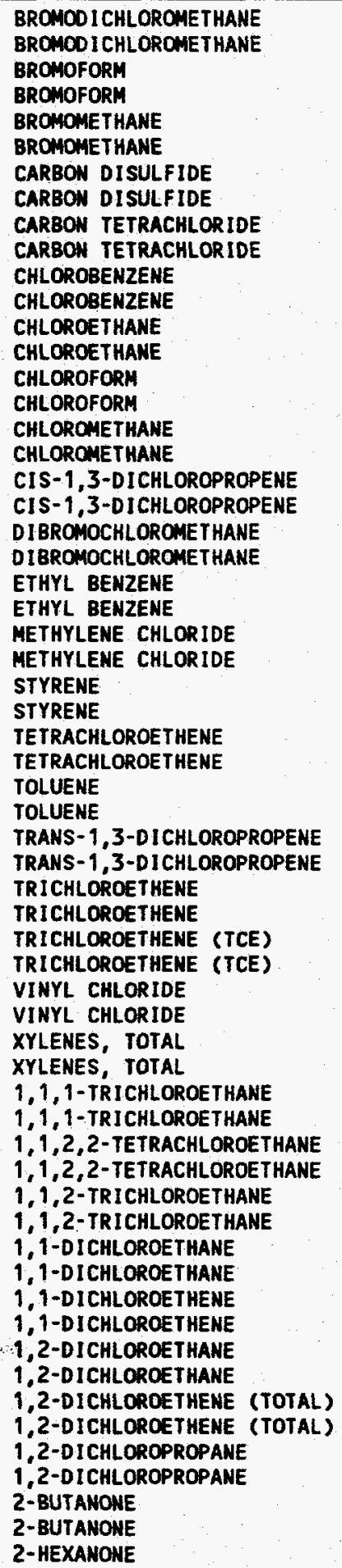 & $\begin{array}{l}\text { ND } \\
\text { ND } \\
\text { ND } \\
\text { ND } \\
\text { ND } \\
\text { ND } \\
\text { ND } \\
\text { ND } \\
\text { ND } \\
\text { ND } \\
\text { ND } \\
\text { ND } \\
\text { ND } \\
\text { ND } \\
\text { ND } \\
\text { ND } \\
\text { ND } \\
\text { ND } \\
\text { ND } \\
\text { ND } \\
\text { ND } \\
\text { ND } \\
\text { ND } \\
\text { ND } \\
\text { (4) } \\
\text { (4) } \\
\text { ND } \\
\text { ND } \\
\text { ND } \\
\text { ND } \\
\text { ND } \\
\text { ND } \\
\text { ND } \\
\text { ND } \\
\text { ND } \\
\text { ND } \\
\text { (1) } \\
\text { 21 } \\
\text { ND } \\
\text { ND } \\
\text { ND } \\
\text { ND } \\
\text { ND } \\
\text { ND } \\
\text { ND } \\
\text { ND } \\
\text { ND } \\
\text { ND } \\
\text { ND } \\
\text { ND } \\
\text { ND } \\
\text { ND } \\
\text { ND } \\
\text { ND } \\
\text { (9) } \\
\text { ND } \\
\text { ND } \\
\text { ND } \\
\text { ND } \\
\text { ND } \\
\text { ND }\end{array}$ & $\begin{array}{l}10 \\
10 \\
10 \\
10 \\
10 \\
10 \\
10 \\
10 \\
10 \\
10 \\
10 \\
10 \\
10 \\
10 \\
10 \\
10 \\
10 \\
10 \\
10 \\
10 \\
10 \\
10 \\
10 \\
10 \\
10 \\
10 \\
10 \\
10 \\
10 \\
10 \\
10 \\
10 \\
10 \\
10 \\
10 \\
10 \\
5 \\
1 \\
10 \\
10 \\
10 \\
10 \\
10 \\
100 \\
10 \\
100 \\
10 \\
100 \\
10 \\
100 \\
10 \\
100 \\
10 \\
100 \\
10 \\
100 \\
10 \\
100 \\
10 \\
100 \\
10\end{array}$ & $\begin{array}{l}U G / L \\
U G / L \\
U G / L \\
U G / L \\
U G / L \\
U G / L \\
U G / L \\
U G / L \\
U G / L \\
U G / L \\
U G / L \\
U G / L \\
U G / L \\
U G / L \\
U G / L \\
U G / L \\
U G / L \\
U G / L \\
U G / L \\
U G / L \\
U G / L \\
U G / L \\
U G / L \\
U G / L \\
U G / L \\
U G / L \\
U G / L \\
U G / L \\
U G / L \\
U G / L \\
U G / L \\
U G / L \\
U G / L \\
U G / L \\
U G / L \\
U G / L \\
U G / L \\
U G / L \\
U G / L \\
U G / L \\
U G / L \\
U G / L \\
U G / L \\
U G / L \\
U G / L \\
U G / L \\
U G / L \\
U G / L \\
U G / L \\
U G / L \\
U G / L \\
U G / L \\
U G / L \\
U G / L \\
U G / L \\
U G / L \\
U G / L \\
U G / L \\
U G / L \\
U G / L \\
U G / L \\
U\end{array}$ & $\begin{array}{l}\text { EPA CLP } \\
\text { EPA CLP } \\
\text { EPA CLP } \\
\text { EPA CLP } \\
\text { EPA CLP } \\
\text { EPA CLP } \\
\text { EPA CLP } \\
\text { EPA CLP } \\
\text { EPA CLP } \\
\text { EPA CLP } \\
\text { EPA CLP } \\
\text { EPA CLP } \\
\text { EPA CLP } \\
\text { EPA CLP } \\
\text { EPA CLP } \\
\text { EPA CLP } \\
\text { EPA CLP } \\
\text { EPA CLP } \\
\text { EPA CLP } \\
\text { EPA CLP } \\
\text { EPA CLP } \\
\text { EPA CLP } \\
\text { EPA CLP } \\
\text { EPA CLP } \\
\text { EPA CLP } \\
\text { EPA CLP } \\
\text { EPA CLP } \\
\text { EPA CLP } \\
\text { EPA CLP } \\
\text { EPA CLP } \\
\text { EPA CLP } \\
\text { EPA CLP } \\
\text { EPA CLP } \\
\text { EPA CLP } \\
\text { EPA CLP } \\
\text { EPA CLP } \\
\text { EPA } 8260 \\
\text { EPA } 8260 \\
\text { EPA CLP } \\
\text { EPA CLP } \\
\text { EPA CLP } \\
\text { EPA CLP } \\
\text { EPA CLP } \\
\text { EPA CLP } \\
\text { EPA CLP } \\
\text { EPA CLP } \\
\text { EPA CLP } \\
\text { EPA CLP } \\
\text { EPA CLP } \\
\text { EPA CLP } \\
\text { EPA CLP } \\
\text { EPA CLP } \\
\text { EPA CLP } \\
\text { EPA CLP } \\
\text { EPA CLP } \\
\text { EPA CLP } \\
\text { EPA CLP } \\
\text { EPA CLP } \\
\text { EPA CLP } \\
\text { EPA CLP } \\
\text { EPA CLP }\end{array}$ \\
\hline
\end{tabular}




\begin{tabular}{|c|c|c|c|c|c|c|}
\hline WSSRAP_ID & DATE_SAM & PARAMETER & CONC & $D L$ & UNITS & METHOD \\
\hline 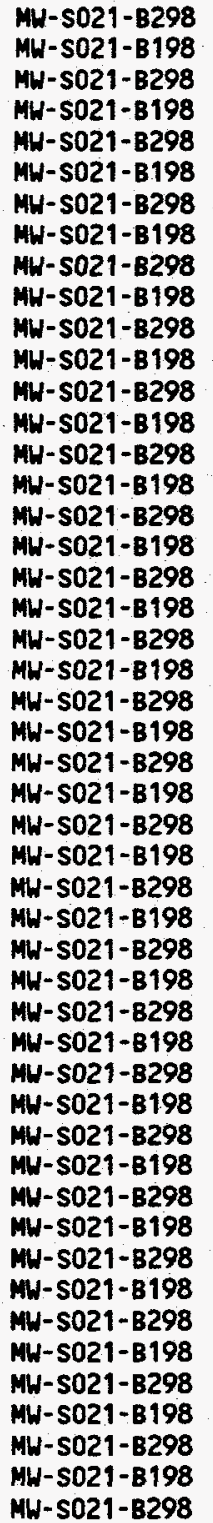 & $\begin{array}{l}03 / 10 / 98 \\
02 / 03 / 98 \\
03 / 10 / 98 \\
02 / 03 / 98 \\
03 / 10 / 98 \\
02 / 03 / 98 \\
03 / 10 / 98 \\
02 / 03 / 98 \\
03 / 10 / 98 \\
02 / 03 / 98 \\
03 / 10 / 98 \\
02 / 03 / 98 \\
03 / 10 / 98 \\
02 / 03 / 98 \\
03 / 10 / 98 \\
02 / 03 / 98 \\
03 / 10 / 98 \\
02 / 03 / 98 \\
03 / 10 / 98 \\
02 / 03 / 98 \\
03 / 10 / 98 \\
02 / 03 / 98 \\
03 / 10 / 98 \\
02 / 03 / 98 \\
03 / 10 / 98 \\
02 / 03 / 98 \\
03 / 10 / 98 \\
02 / 03 / 98 \\
03 / 10 / 98 \\
02 / 03 / 98 \\
03 / 10 / 98 \\
02 / 03 / 98 \\
03 / 10 / 98 \\
02 / 03 / 98 \\
03 / 10 / 98 \\
02 / 03 / 98 \\
03 / 10 / 98 \\
02 / 03 / 98 \\
03 / 10 / 98 \\
02 / 03 / 98 \\
03 / 10 / 98 \\
02 / 03 / 98 \\
03 / 10 / 98 \\
02 / 03 / 98 \\
03 / 10 / 98 \\
02 / 03 / 98 \\
03 / 10 / 98 \\
02 / 03 / 98 \\
03 / 10 / 98\end{array}$ & 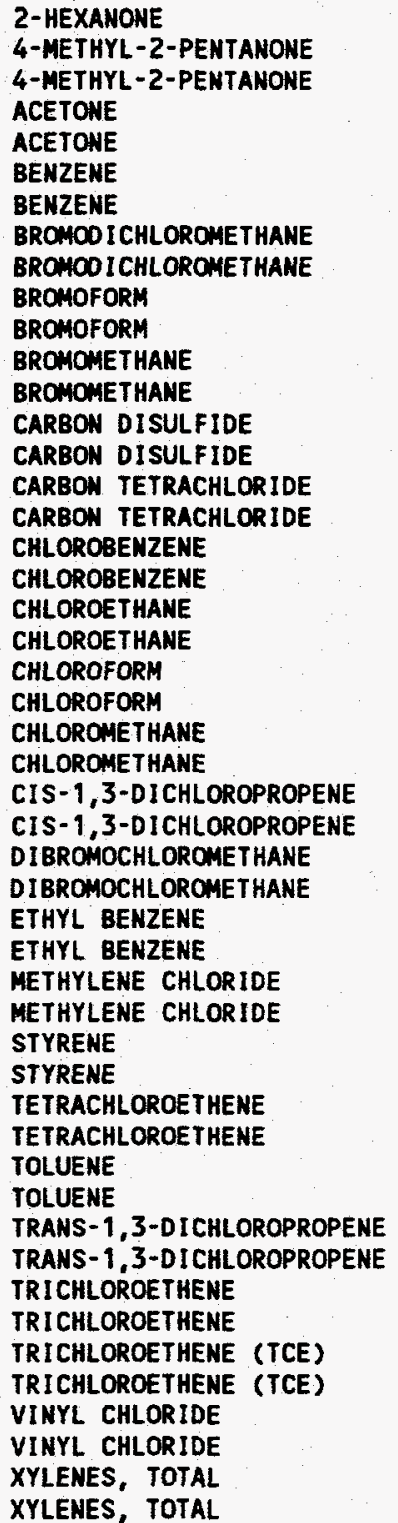 & $\begin{array}{l}\text { ND } \\
\text { ND } \\
\text { (3) } \\
\text { ND } \\
\text { ND } \\
\text { ND } \\
\text { ND } \\
\text { ND } \\
\text { ND } \\
\text { ND } \\
\text { ND } \\
\text { ND } \\
\text { ND } \\
\text { ND } \\
\text { ND } \\
\text { ND } \\
\text { ND } \\
\text { ND } \\
\text { ND } \\
\text { ND } \\
\text { ND } \\
\text { ND } \\
\text { ND } \\
\text { ND } \\
\text { ND } \\
\text { ND } \\
\text { ND } \\
\text { ND } \\
\text { (4) } \\
\text { NB } \\
\text { ND } \\
\text { ND } \\
\text { ND } \\
\text { ND } \\
\text { ND } \\
\text { ND } \\
\text { ND } \\
\text { C20 } \\
\text { 38O } \\
\text { ND } \\
\text { 160 } \\
\text { ND } \\
\text { ND } \\
\text { ND }\end{array}$ & $\begin{array}{l}100 \\
10 \\
100 \\
10 \\
100 \\
10 \\
100 \\
10 \\
100 \\
10 \\
100 \\
10 \\
100 \\
10 \\
100 \\
10 \\
100 \\
10 \\
100 \\
10 \\
100 \\
10 \\
100 \\
10 \\
100 \\
10 \\
100 \\
10 \\
100 \\
10 \\
100 \\
10 \\
100 \\
10 \\
100 \\
10 \\
100 \\
10 \\
100 \\
10 \\
100 \\
50 \\
100 \\
5 \\
1 \\
10 \\
100 \\
10 \\
100\end{array}$ & $\begin{array}{l}U G / L \\
U G / L \\
U G / L \\
U G / L \\
U G / L \\
U G / L \\
U G / L \\
U G / L \\
U G / L \\
U G / L \\
U G / L \\
U G / L \\
U G / L \\
U G / L \\
U G / L \\
U G / L \\
U G / L \\
U G / L \\
U G / L \\
U G / L \\
U G / L \\
U G / L \\
U G / L \\
U G / L \\
U G / L \\
U G / L \\
U G / L \\
U G / L \\
U G / L \\
U G / L \\
U G / L \\
U G / L \\
U G / L \\
U G / L \\
U G / L \\
U G / L \\
U G / L \\
U G / L \\
U G / L \\
U G / L \\
U G / L \\
U G / L \\
U G / L \\
U G / L \\
U G / L \\
U G / L \\
U G / L \\
U G / L \\
U G / L\end{array}$ & $\begin{array}{l}\text { EPA CLP } \\
\text { EPA CLP } \\
\text { EPA CLP } \\
\text { EPA CLP } \\
\text { EPA CLP } \\
\text { EPA CLP } \\
\text { EPA CLP } \\
\text { EPA CLP } \\
\text { EPA CLP } \\
\text { EPA CLP } \\
\text { EPA CLP } \\
\text { EPA CLP } \\
\text { EPA CLP } \\
\text { EPA CLP } \\
\text { EPA CLP } \\
\text { EPA CLP } \\
\text { EPA CLP } \\
\text { EPA CLP } \\
\text { EPA CLP } \\
\text { EPA CLP } \\
\text { EPA CLP } \\
\text { EPA CLP } \\
\text { EPA CLP } \\
\text { EPA CLP } \\
\text { EPA CLP } \\
\text { EPA CLP } \\
\text { EPA CLP } \\
\text { EPA CLP } \\
\text { EPA CLP } \\
\text { EPA CLP } \\
\text { EPA CLP } \\
\text { EPA CLP } \\
\text { EPA CLP } \\
\text { EPA CLP } \\
\text { EPA CLP } \\
\text { EPA CLP } \\
\text { EPA CLP } \\
\text { EPA CLP } \\
\text { EPA CLP } \\
\text { EPA CLP } \\
\text { EPA CLP } \\
\text { EPA CLP } \\
\text { EPA CLP } \\
\text { EPA } 8260 \\
\text { EPA } 8260 \\
\text { EPA CLP } \\
\text { EPA CLP } \\
\text { EPA CLP } \\
\text { EPA CLP }\end{array}$ \\
\hline
\end{tabular}




\section{SURFACE WATER}


SURFACE WATER

DATA MERGED DURING SECOND QUARTER 1998

\begin{tabular}{|c|c|c|c|c|c|c|}
\hline HSSRAP_ID & DATE_SAM & PARAMETER & CONC & $\mathrm{DL}$ & UNITS & METHOD \\
\hline 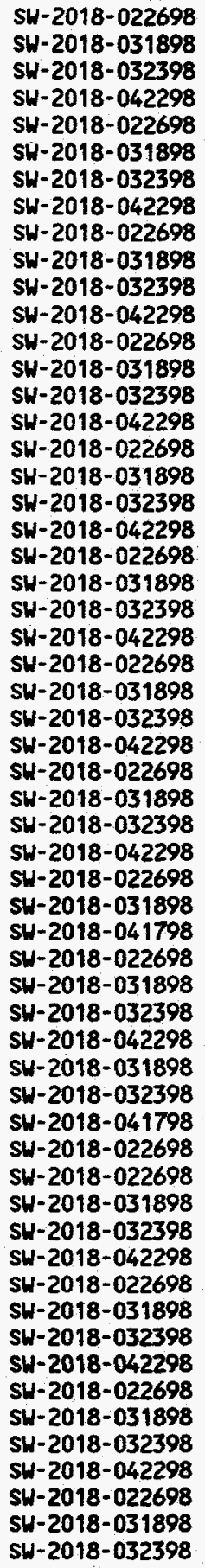 & $\begin{array}{l}02 / 26 / 98 \\
03 / 18 / 98 \\
03 / 23 / 98 \\
04 / 22 / 98 \\
02 / 26 / 98 \\
03 / 18 / 98 \\
03 / 23 / 98 \\
04 / 22 / 98 \\
02 / 26 / 98 \\
03 / 18 / 98 \\
03 / 23 / 98 \\
04 / 22 / 98 \\
02 / 26 / 98 \\
03 / 18 / 98 \\
03 / 23 / 98 \\
04 / 22 / 98 \\
02 / 26 / 98 \\
03 / 18 / 98 \\
03 / 23 / 98 \\
04 / 22 / 98 \\
02 / 26 / 98 \\
03 / 18 / 98 \\
03 / 23 / 98 \\
04 / 22 / 98 \\
02 / 26 / 98 \\
03 / 18 / 98 \\
03 / 23 / 98 \\
04 / 22 / 98 \\
02 / 26 / 98 \\
03 / 18 / 98 \\
03 / 23 / 98 \\
04 / 22 / 98 \\
02 / 26 / 98 \\
03 / 18 / 98 \\
04 / 17 / 98 \\
02 / 26 / 98 \\
03 / 18 / 98 \\
03 / 23 / 98 \\
04 / 22 / 98 \\
03 / 18 / 98 \\
03 / 23 / 98 \\
04 / 17 / 98 \\
02 / 26 / 98 \\
02 / 26 / 98 \\
03 / 18 / 98 \\
03 / 23 / 98 \\
04 / 22 / 98 \\
02 / 26 / 98 \\
03 / 18 / 98 \\
03 / 23 / 98 \\
04 / 22 / 98 \\
02 / 26 / 98 \\
03 / 18 / 98 \\
03 / 23 / 98 \\
04 / 22 / 98 \\
02 / 26 / 98 \\
03 / 18 / 98 \\
03 / 23 / 98\end{array}$ & 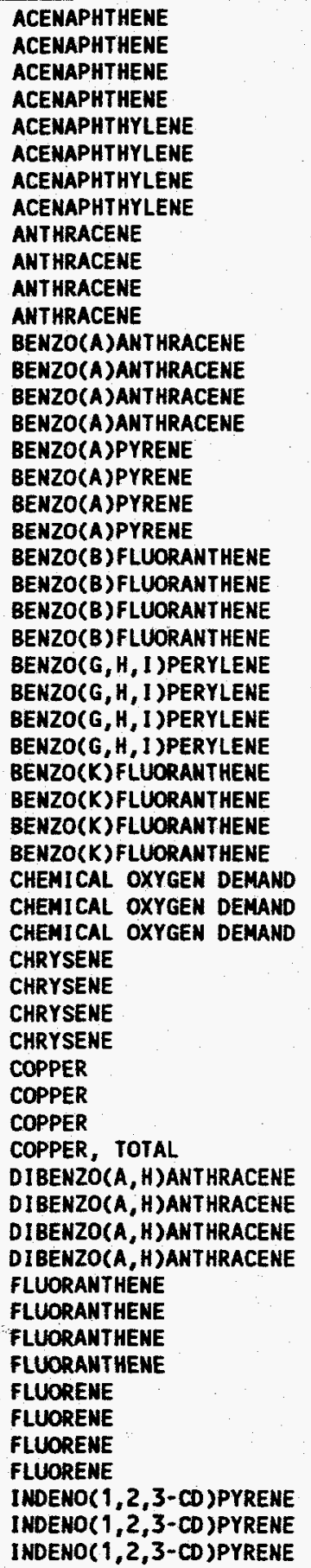 & $\begin{array}{l}\text { ND } \\
\text { ND } \\
\text { ND } \\
\text { ND } \\
\text { ND } \\
\text { ND } \\
\text { ND } \\
\text { ND } \\
\text { ND } \\
\text { ND } \\
\text { ND } \\
\text { ND } \\
\text { ND } \\
\text { ND } \\
\text { ND } \\
\text { ND } \\
\text { ND } \\
\text { ND } \\
\text { ND } \\
\text { ND } \\
\text { ND } \\
\text { ND } \\
\text { ND } \\
\text { ND } \\
\text { ND } \\
\text { ND } \\
\text { ND } \\
\text { ND } \\
200 \\
144 \\
135 \\
\text { ND } \\
\text { ND } \\
\text { ND } \\
\text { ND } \\
\text { ND } 1 \\
\text { ND } \\
\text { ND } \\
\text { ND } \\
\text { ND } \\
\text { ND } \\
12 \\
\text { ND } \\
\text { ND } \\
\text { ND } \\
\text { ND } \\
\text { ND } \\
\text { ND } \\
\text { ND } \\
\text { No }\end{array}$ & $\begin{array}{l}5.0 \\
4.8 \\
4.9 \\
1.1 \\
5.0 \\
4.8 \\
4.9 \\
1.1 \\
5.0 \\
4.8 \\
4.9 \\
1.1 \\
5.0 \\
4.8 \\
4.9 \\
1.1 \\
5.0 \\
4.8 \\
4.9 \\
1.1 \\
5.0 \\
4.8 \\
4.9 \\
1.1 \\
5.0 \\
4.8 \\
4.9 \\
1.1 \\
5.0 \\
4.8 \\
4.9 \\
1.1 \\
10 \\
5.00 \\
5.36 \\
5.0 \\
4.8 \\
4.9 \\
1.1 \\
3.1 \\
3.1 \\
1.2 \\
4.0 \\
5.0 \\
4.8 \\
4.9 \\
1.1 \\
5.0 \\
4.8 \\
4.9 \\
1.1 \\
4.0 \\
4.8 \\
\end{array}$ & $\begin{array}{l}\text { UG/L } \\
U G / L \\
U G / L \\
U G / L \\
U G / L \\
U G / L \\
U G / L \\
U G / L \\
U G / L \\
U G / L \\
U G / L \\
U G / L \\
U G / L \\
U G / L \\
U G / L \\
U G / L \\
U G / L \\
U G / L \\
U G / L \\
U G / L \\
U G / L \\
U G / L \\
U G / L \\
U G / L \\
U G / L \\
U G / L \\
U G / L \\
U G / L \\
U G / L \\
U G / L \\
U G / L \\
U G / L \\
\text { UG/L } \\
\text { UG/L } \\
\text { UG/L } \\
U G / L \\
U G / L \\
U G / L \\
U G / L \\
U G / L \\
U G / L \\
U G / L \\
U G / L \\
U G / L \\
U G / L \\
U G / L \\
U G / L \\
U G / L \\
U G / L \\
U G / L \\
U G / L \\
U G / L \\
U G / L \\
U G / L \\
U G / L \\
U G / L \\
U G / L \\
U G / L\end{array}$ & $\begin{array}{l}\text { EPA } 8270 A \\
\text { EPA } 8310 \\
\text { EPA } 8310 \\
\text { EPA } 8270 A \\
\text { EPA } 8270 A \\
\text { EPA } 8310 \\
\text { EPA } 8310 \\
\text { EPA } 8270 A \\
\text { EPA } 8270 A \\
\text { EPA } 8310 \\
\text { EPA } 8310 \\
\text { EPA } 8270 A \\
\text { EPA } 8270 A \\
\text { EPA } 8310 \\
\text { EPA } 8310 \\
\text { EPA } 8270 A \\
\text { EPA } 8270 A \\
\text { EPA } 8310 \\
\text { EPA } 8310 \\
\text { EPA } 8270 A \\
\text { EPA } 8270 A \\
\text { EPA } 8310 \\
\text { EPA } 8310 \\
\text { EPA } 8270 A \\
\text { EPA } 8270 A \\
\text { EPA } 8310 \\
\text { EPA } 8310 \\
\text { EPA } 8270 A \\
\text { EPA } 8270 A \\
\text { EPA } 8310 \\
\text { EPA } 8310 \\
\text { EPA } 8270 A \\
\text { EPA } 410.0 \\
\text { EPA } 410.4 \\
\text { EPA } 410.4 \\
\text { EPA } 8270 A \\
\text { EPA } 8310 \\
\text { EPA } 8310 \\
\text { EPA } 8270 A \\
\text { EPA } \mathrm{CLP} \\
\text { EPA } \mathrm{CLP} \\
\text { EPA } 6010 A \\
\text { EPA } 200.7 \\
\text { EPA } 8270 A \\
\text { EPA } 8310 \\
\text { EPA } 8310 \\
\text { EPA } 8270 A \\
\text { EPA } 8270 A \\
\text { EPA } 8310 \\
\text { EPA } 8310 \\
\text { EPA } 8270 A \\
\text { EPA } 8270 A \\
\text { EPA } 8310 \\
\text { EPA } 8310 \\
\text { EPA } 8270 A \\
\text { EPA } 8270 A \\
\text { EPA } 8310 \\
\text { EPA } 8310\end{array}$ \\
\hline
\end{tabular}




\begin{tabular}{|c|c|c|c|c|c|c|}
\hline WSSRAP_ID & DATE_SAM & PARAMETER & CONC & DL & UNITS & METHOD \\
\hline $\begin{array}{l}\text { SW-2018-042298 } \\
\text { SW-2018-022698 } \\
\text { SW-2018-031898 } \\
\text { SW-2018-032398 } \\
\text { SW-2018-042298 } \\
\text { SW-2018-022698 } \\
\text { SW-2018-031898 } \\
\text { SW-2018-041798 } \\
\text { SW-2018-022698 } \\
\text { SW-2018-031898 } \\
\text { SW-2018-032398 } \\
\text { SW-2018-042298 } \\
\text { SW-2018-022698 } \\
\text { SW-2018-031898 } \\
\text { SW-2018-032398 } \\
\text { SW-2018-042298 } \\
\text { SW-2018-031898 } \\
\text { SW-2018-032398 } \\
\text { SW-2018-041798 } \\
\text { SW-2018-022698 } \\
\text { SW-2025-031098 }\end{array}$ & $\begin{array}{l}04 / 22 / 98 \\
02 / 26 / 98 \\
03 / 18 / 98 \\
03 / 23 / 98 \\
04 / 22 / 98 \\
02 / 26 / 98 \\
03 / 18 / 98 \\
04 / 17 / 98 \\
02 / 26 / 98 \\
03 / 18 / 98 \\
03 / 23 / 98 \\
04 / 22 / 98 \\
02 / 26 / 98 \\
03 / 18 / 98 \\
03 / 23 / 98 \\
04 / 22 / 98 \\
03 / 18 / 98 \\
03 / 23 / 98 \\
04 / 17 / 98 \\
02 / 26 / 98 \\
03 / 10 / 98\end{array}$ & $\begin{array}{l}\text { INDENO(1,2,3-CD)PYRENE } \\
\text { NAPHTHALENE } \\
\text { NAPHTHALENE } \\
\text { NAPHTHALENE } \\
\text { NAPHTHALENE } \\
\text { NITRATE-N } \\
\text { NITRATE-N } \\
\text { NITRATE-N } \\
\text { PHENANTHRENE } \\
\text { PHENANTHRENE } \\
\text { PHENANTHRENE } \\
\text { PHENANTHRENE } \\
\text { PYRENE } \\
\text { PYRENE } \\
\text { PYRENE } \\
\text { PYRENE } \\
\text { ZINC } \\
\text { ZINC } \\
\text { ZINC } \\
\text { ZINC, TOTAL } \\
\text { 2,4-DINITROTOLUENE }\end{array}$ & $\begin{array}{l}\text { ND } \\
\text { ND } \\
\text { ND } \\
\text { ND } \\
\text { ND } \\
7.2 \\
14.7 \\
4.98 \\
\text { ND } \\
\text { ND } \\
\text { ND } \\
\text { ND } \\
\text { ND } \\
\text { ND } \\
\text { ND } \\
20.6 \\
13.7 \\
9.5 \\
23 \\
\text { ND }\end{array}$ & $\begin{array}{l}1.1 \\
5.0 \\
4.8 \\
4.9 \\
1.1 \\
1.0 \\
1.25 \\
0.014 \\
5.0 \\
4.8 \\
4.9 \\
1.1 \\
5.0 \\
4.8 \\
4.9 \\
1.1 \\
1.3 \\
1.3 \\
0.3 \\
20 \\
0.20\end{array}$ & $\begin{array}{l}U G / L \\
U G / L \\
U G / L \\
U G / L \\
U G / L \\
M G / L \\
M G / L \\
M G / L \\
U G / L \\
U G / L \\
U G / L \\
U G / L \\
U G / L \\
U G / L \\
U G / L \\
U G / L \\
U G / L \\
U G / L \\
U G / L \\
U G / L \\
U G / L\end{array}$ & $\begin{array}{l}\text { EPA } 8270 A \\
\text { EPA } 8270 A \\
\text { EPA } 8310 \\
\text { EPA } 8310 \\
\text { EPA } 8270 A \\
\text { EPA } 300.0 \\
\text { EPA } 353.1 \\
\text { EPA } 353.1 \\
\text { EPA } 8270 A \\
\text { EPA } 8310 \\
\text { EPA } 8310 \\
\text { EPA } 8270 A \\
\text { EPA } 8270 A \\
\text { EPA } 8310 \\
\text { EPA } 8310 \\
\text { EPA } 8270 A \\
\text { EPA CLP } \\
\text { EPA CLP } \\
\text { EPA } 6010 A \\
\text { EPA } 200.7 \\
\text { SWB46 } 8330\end{array}$ \\
\hline
\end{tabular}




\section{DOWNSTREAM SURFACE WATER URANIUM RESULTS Second Quarter 1998}

\begin{tabular}{|c|c|c|c|c|}
\hline WSSRAP ID & DATE & PARAMETER & CONCENTRATION & UNITS \\
\hline SW-1003-Q298 & $4 / 17 / 98$ & Uranium, Total & 32.55 & $\mathrm{pCi} / 1$ \\
\hline SW-1004-Q298 & $4 / 17 / 98$ & Uranium, Total & 39.26 & $\mathrm{pCi} / 1$ \\
\hline SW-1005-Q298 & $4 / 17 / 98$ & Uranium, Total & 29.65 & $\mathrm{pCi} / 1$ \\
\hline SW-1007-Q298 & $4 / 17 / 98$ & Uranium, Total & 10.11 & $\mathrm{pCi} / 1$ \\
\hline SW-1009-Q298 & $4 / 17 / 98$ & Uranium, Total & 9.85 & $\mathrm{pCi} / 1$ \\
\hline SW-1010-Q298 & $4 / 17 / 98$ & Uranium, Total & 28.46 & $\mathrm{pCi} / 1$ \\
\hline SW-2001-051298 & $5 / 12 / 98$ & Uranium, Total & 1.9 & $\mathrm{pCi} / 1$ \\
\hline SW-2002-051298 & $5 / 12 / 98$ & Uranium, Total & 21.8 & $\mathrm{pCi} / 1$ \\
\hline SW-2003-051298 & $5 / 12 / 98$ & Uranium, Total & 7.7 & $\mathrm{pCi} / 1$ \\
\hline SW-2004-051298 & $5 / 12 / 98$ & Uranium, Total & 10.9 & $\mathrm{pCi} / 1$ \\
\hline SW-2005-051298 & $5 / 12 / 98$ & Uranium, Total & 37.2 & $\mathrm{pCi} / 1$ \\
\hline SW-2012-051298 & $5 / 12 / 98$ & Uranium, Total & 8.5 & $\mathrm{pCi} / 1$ \\
\hline SW-2016-051298 & $5 / 12 / 98$ & Uranium, Total & 2.1 & $\mathrm{pCi} / 1$ \\
\hline
\end{tabular}


4

2.

SPRINGS 


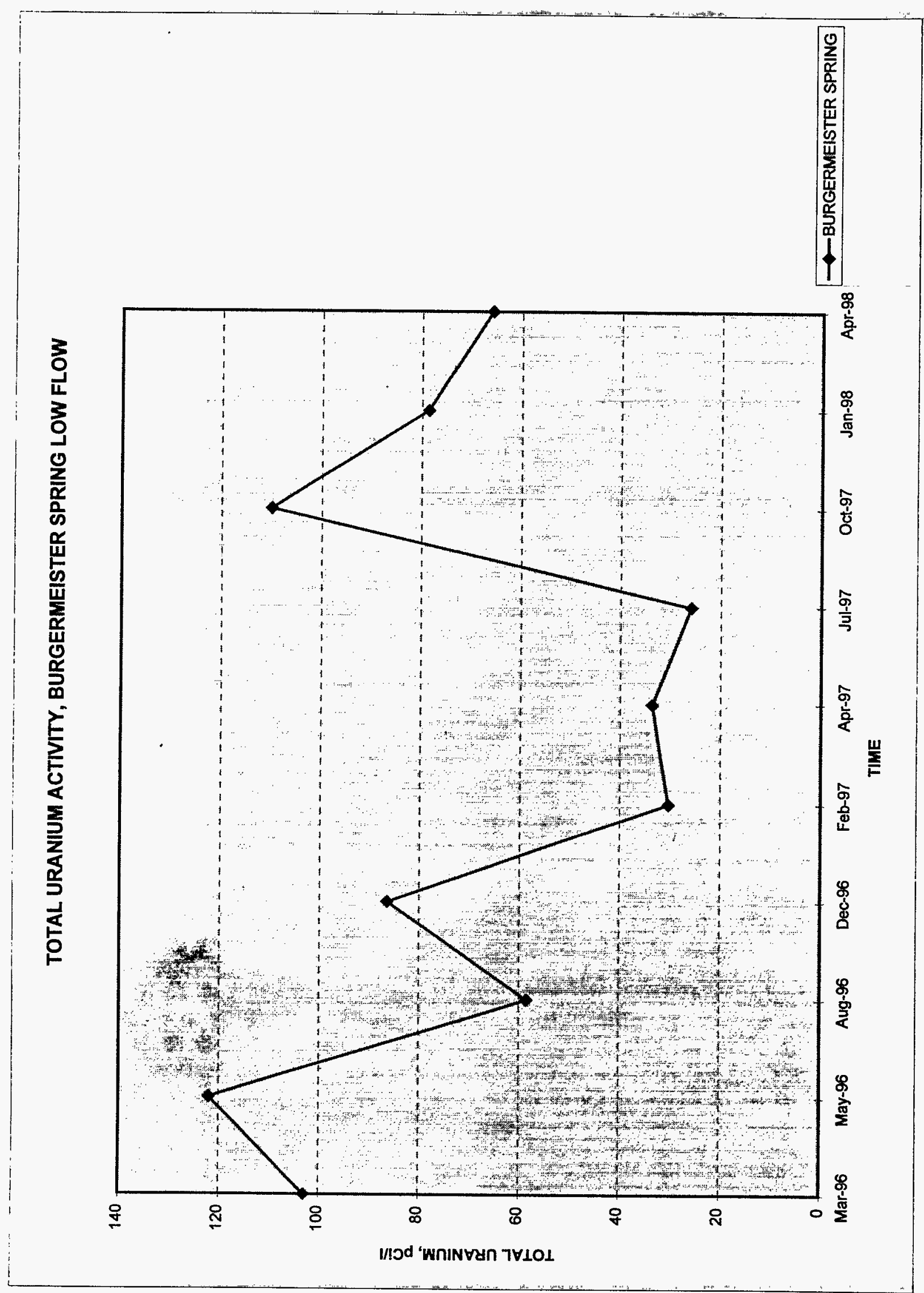


SPRINGS

DATA MERGED DURING SECOND QUARTER 1998

\begin{tabular}{|c|c|c|c|c|c|c|}
\hline WSSRAP_ID & DATE_SAM & PARAMETER & CONC & DL & UNITS & METHOD \\
\hline 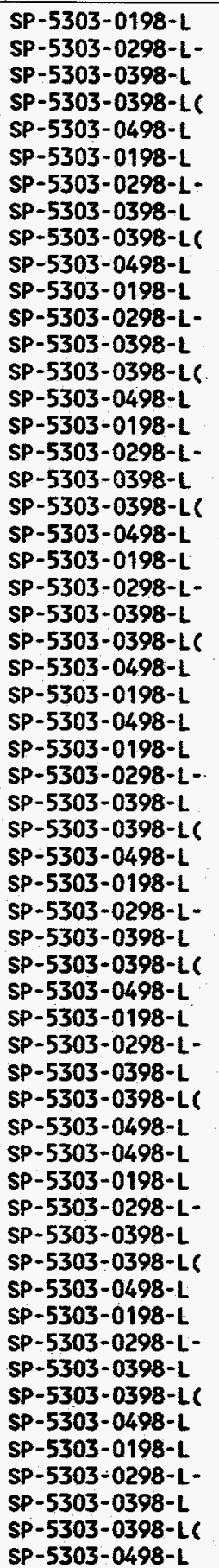 & $\begin{array}{l}01 / 15 / 98 \\
02 / 25 / 98 \\
03 / 16 / 98 \\
03 / 16 / 98 \\
04 / 20 / 98 \\
01 / 15 / 98 \\
02 / 25 / 98 \\
03 / 16 / 98 \\
03 / 16 / 98 \\
04 / 20 / 98 \\
01 / 15 / 98 \\
02 / 25 / 98 \\
03 / 16 / 98 \\
03 / 16 / 98 \\
04 / 20 / 98 \\
01 / 15 / 98 \\
02 / 25 / 98 \\
03 / 16 / 98 \\
03 / 16 / 98 \\
04 / 20 / 98 \\
01 / 15 / 98 \\
02 / 25 / 98 \\
03 / 16 / 98 \\
03 / 16 / 98 \\
04 / 20 / 98 \\
01 / 15 / 98 \\
04 / 20 / 98 \\
01 / 15 / 98 \\
02 / 25 / 98 \\
03 / 16 / 98 \\
03 / 16 / 98 \\
04 / 20 / 98 \\
01 / 15 / 98 \\
02 / 25 / 98 \\
03 / 16 / 98 \\
03 / 16 / 98 \\
04 / 20 / 98 \\
01 / 15 / 98 \\
02 / 25 / 98 \\
03 / 16 / 98 \\
03 / 16 / 98 \\
04 / 20 / 98 \\
04 / 20 / 98 \\
01 / 15 / 98 \\
02 / 25 / 98 \\
03 / 16 / 98 \\
03 / 16 / 98 \\
04 / 20 / 98 \\
01 / 15 / 98 \\
02 / 25 / 98 \\
03 / 16 / 98 \\
03 / 16 / 98 \\
04 / 20 / 98 \\
01 / 15 / 98 \\
02 / 25 / 98 \\
03 / 16 / 98 \\
03 / 16 / 98 \\
04 / 20 / 98\end{array}$ & 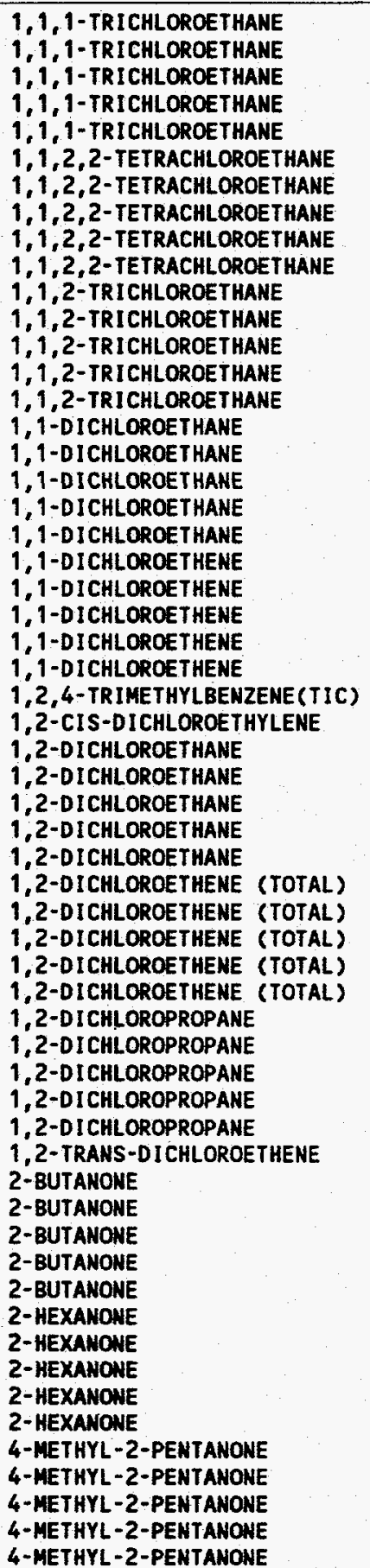 & 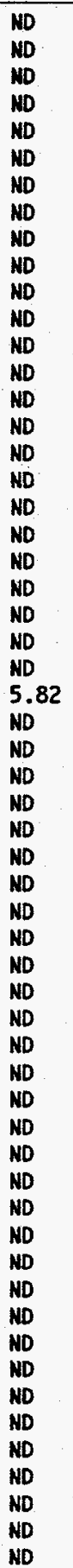 & $\begin{array}{l}10 \\
10 \\
10 \\
10 \\
10.0 \\
10 \\
10 \\
10 \\
10 \\
10.0 \\
10 \\
10 \\
10 \\
10 \\
10.0 \\
10 \\
10 \\
10 \\
10 \\
10.0 \\
10 \\
10 \\
10 \\
10 \\
10.0 \\
0 \\
10.0 \\
10 \\
10 \\
10 \\
10 \\
10.0 \\
10 \\
10 \\
10 \\
10 \\
10.0 \\
10 \\
10 \\
10 \\
10 \\
10.0 \\
10.0 \\
10 \\
10 \\
10 \\
10 \\
10.0 \\
10 \\
10 \\
10 \\
10 \\
10.0 \\
10 \\
10 \\
10 \\
10 \\
10.0\end{array}$ & $\begin{array}{l}\text { UG/L } \\
U G / L \\
U G / L \\
U G / L \\
U G / L \\
U G / L \\
U G / L \\
U G / L \\
U G / L \\
U G / L \\
U G / L \\
U G / L \\
U G / L \\
U G / L \\
U G / L \\
U G / L \\
U G / L \\
U G / L \\
U G / L \\
U G / L \\
U G / L \\
U G / L \\
U G / L \\
U G / L \\
U G / L \\
U G / L \\
U G / L \\
U G / L \\
U G / L \\
U G / L \\
U G / L \\
U G / L \\
U G / L \\
U G / L \\
U G / L \\
U G / L \\
U G / L \\
U G / L \\
U G / L \\
U G / L \\
U G / L \\
U G / L \\
U G / L \\
U G / L \\
U G / L \\
U G / L \\
U G / L \\
U G / L \\
U G / L \\
U G / L \\
U G / L \\
U G / L \\
U G / L \\
U G / L \\
U G / L \\
U G / L \\
U G / L \\
U G / L\end{array}$ & 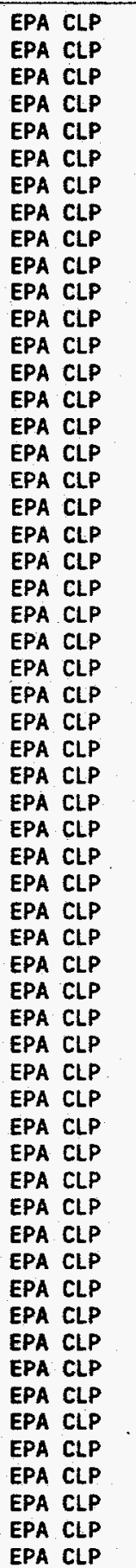 \\
\hline
\end{tabular}




\begin{tabular}{|c|c|c|c|c|c|c|}
\hline WSSRAP_ID & DATE_SAM & PARAMETER & CONC & $D L$ & UNITS & METHOD \\
\hline 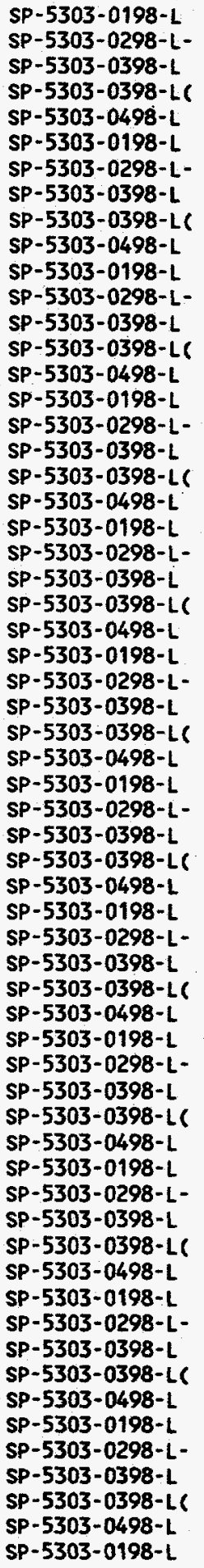 & $\begin{array}{l}01 / 15 / 98 \\
02 / 25 / 98 \\
03 / 16 / 98 \\
03 / 16 / 98 \\
04 / 20 / 98 \\
01 / 15 / 98 \\
02 / 25 / 98 \\
03 / 16 / 98 \\
03 / 16 / 98 \\
04 / 20 / 98 \\
01 / 15 / 98 \\
02 / 25 / 98 \\
03 / 16 / 98 \\
03 / 16 / 98 \\
04 / 20 / 98 \\
01 / 15 / 98 \\
02 / 25 / 98 \\
03 / 16 / 98 \\
03 / 16 / 98 \\
04 / 20 / 98 \\
01 / 15 / 98 \\
02 / 25 / 98 \\
03 / 16 / 98 \\
03 / 16 / 98 \\
04 / 20 / 98 \\
01 / 15 / 98 \\
02 / 25 / 98 \\
03 / 16 / 98 \\
03 / 16 / 98 \\
04 / 20 / 98 \\
01 / 15 / 98 \\
02 / 25 / 98 \\
03 / 16 / 98 \\
03 / 16 / 98 \\
04 / 20 / 98 \\
01 / 15 / 98 \\
02 / 25 / 98 \\
03 / 16 / 98 \\
03 / 16 / 98 \\
04 / 20 / 98 \\
01 / 15 / 98 \\
02 / 25 / 98 \\
03 / 16 / 98 \\
03 / 16 / 98 \\
04 / 20 / 98 \\
01 / 15 / 98 \\
02 / 25 / 98 \\
03 / 16 / 98 \\
03 / 16 / 98 \\
04 / 20 / 98 \\
01 / 15 / 98 \\
02 / 25 / 98 \\
03 / 16 / 98 \\
03 / 16 / 98 \\
04 / 20 / 98 \\
01 / 15 / 98 \\
02 / 25 / 98 \\
03 / 16 / 98 \\
03 / 16 / 98 \\
04 / 20 / 98 \\
01 / 15 / 98\end{array}$ & 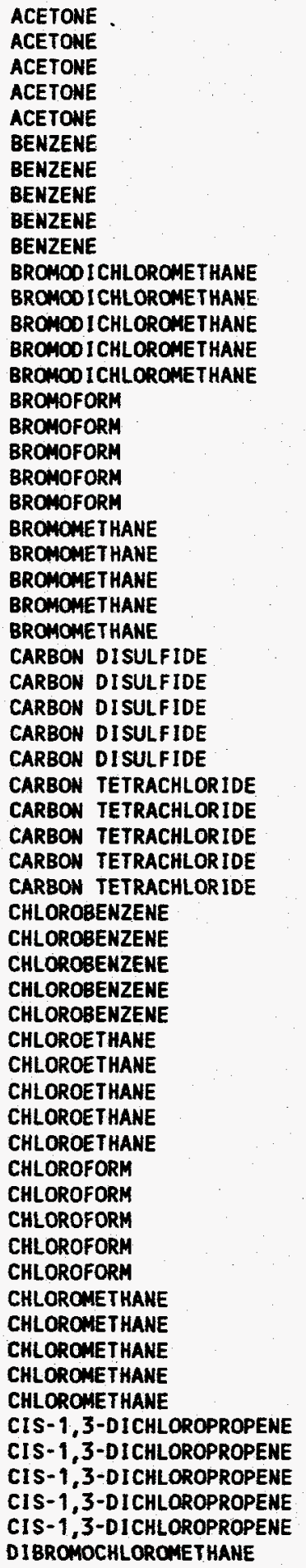 & $\begin{array}{l}12 \\
\text { (4) } \\
\text { C } \\
\text { ND } \\
\text { ND } \\
\text { ND } \\
\text { ND } \\
\text { ND } \\
\text { ND } \\
\text { ND } \\
\text { ND } \\
\text { ND } \\
\text { ND } \\
\text { ND } \\
\text { ND } \\
\text { ND } \\
\text { ND } \\
\text { ND } \\
\text { ND } \\
\text { ND } \\
\text { ND } \\
\text { ND } \\
\text { ND } \\
\text { ND } \\
\text { ND } \\
\text { ND } \\
\text { ND } \\
\text { ND } \\
\text { ND } \\
\text { ND } \\
\text { ND } \\
\text { ND } \\
\text { ND } \\
\text { ND } \\
\text { ND } \\
\text { ND } \\
\text { ND } \\
\text { ND } \\
\text { ND } \\
\text { ND } \\
\text { ND } \\
\text { ND } \\
\text { ND } \\
\text { ND } \\
\text { ND } \\
\text { NO }\end{array}$ & $\begin{array}{l}10 \\
10 \\
10 \\
10 \\
10.0 \\
10 \\
10 \\
10 \\
10 \\
10.0 \\
10 \\
10 \\
10 \\
10 \\
10.0 \\
10 \\
10 \\
10 \\
10 \\
10.0 \\
10 \\
10 \\
10 \\
10 \\
10.0 \\
10 \\
10 \\
10 \\
10 \\
10.0 \\
10 \\
10 \\
10 \\
10 \\
10.0 \\
10 \\
10 \\
10 \\
10 \\
10.0 \\
10 \\
10 \\
10 \\
10 \\
10.0 \\
10 \\
10 \\
10 \\
10 \\
10.0 \\
10 \\
10 \\
10 \\
10 \\
10.0 \\
10 \\
10 \\
10 \\
10 \\
10.0 \\
10\end{array}$ & $\begin{array}{l}U G / L \\
U G / L \\
U G / L \\
U G / L \\
U G / L \\
U G / L \\
U G / L \\
U G / L \\
U G / L \\
U G / L \\
U G / L \\
U G / L \\
U G / L \\
U G / L \\
U G / L \\
U G / L \\
U G / L \\
U G / L \\
U G / L \\
U G / L \\
U G / L \\
U G / L \\
U G / L \\
U G / L \\
U G / L \\
U G / L \\
U G / L \\
U G / L \\
U G / L \\
U G / L \\
U G / L \\
U G / L \\
U G / L \\
U G / L \\
U G / L \\
U G / L \\
U G / L \\
U G / L \\
U G / L \\
U G / L \\
U G / L \\
U G / L \\
U G / L \\
U G / L \\
U G / L \\
U G / L \\
U G / L \\
U G / L \\
U G / L \\
U G / L \\
U G / L \\
U G / L \\
U G / L \\
U G / L \\
U G / L \\
U G / L \\
U G / L \\
U G / L \\
U G / L \\
U G / L \\
U G / L \\
U\end{array}$ & 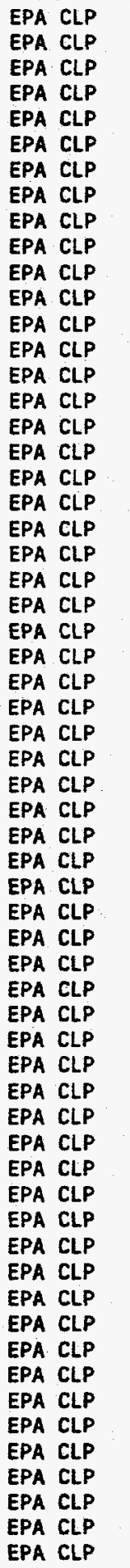 \\
\hline
\end{tabular}




\begin{tabular}{|c|c|c|c|c|c|c|}
\hline USSRAP_ID & DATE_SAM & PARAMETER & CONC & DL & UNITS & METHOD \\
\hline 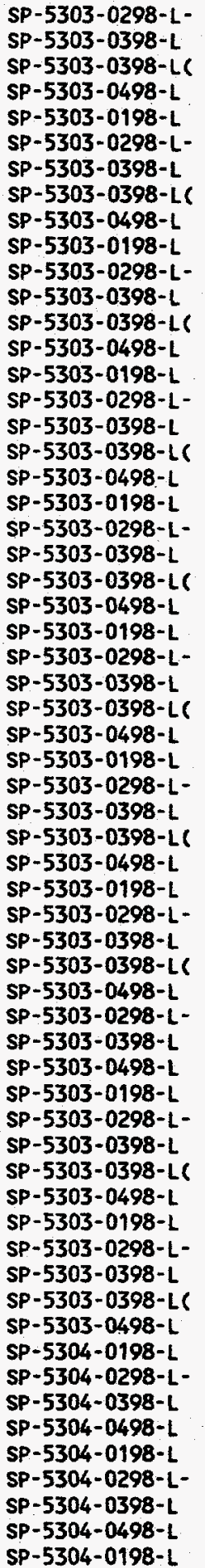 & $\begin{array}{l}02 / 25 / 98 \\
03 / 16 / 98 \\
03 / 16 / 98 \\
04 / 20 / 98 \\
01 / 15 / 98 \\
02 / 25 / 98 \\
03 / 16 / 98 \\
03 / 16 / 98 \\
04 / 20 / 98 \\
01 / 15 / 98 \\
02 / 25 / 98 \\
03 / 16 / 98 \\
03 / 16 / 98 \\
04 / 20 / 98 \\
01 / 15 / 98 \\
02 / 25 / 98 \\
03 / 16 / 98 \\
03 / 16 / 98 \\
04 / 20 / 98 \\
01 / 15 / 98 \\
02 / 25 / 98 \\
03 / 16 / 98 \\
03 / 16 / 98 \\
04 / 20 / 98 \\
01 / 15 / 98 \\
02 / 25 / 98 \\
03 / 16 / 98 \\
03 / 16 / 98 \\
04 / 20 / 98 \\
01 / 15 / 98 \\
02 / 25 / 98 \\
03 / 16 / 98 \\
03 / 16 / 98 \\
04 / 20 / 98 \\
01 / 15 / 98 \\
02 / 25 / 98 \\
03 / 16 / 98 \\
03 / 16 / 98 \\
04 / 20 / 98 \\
02 / 25 / 98 \\
03 / 16 / 98 \\
04 / 20 / 98 \\
01 / 15 / 98 \\
02 / 25 / 98 \\
03 / 16 / 98 \\
03 / 16 / 98 \\
04 / 20 / 98 \\
01 / 15 / 98 \\
02 / 25 / 98 \\
03 / 16 / 98 \\
03 / 16 / 98 \\
04 / 20 / 98 \\
01 / 15 / 98 \\
02 / 25 / 98 \\
03 / 16 / 98 \\
04 / 20 / 98 \\
01 / 15 / 98 \\
02 / 25 / 98 \\
03 / 16 / 98 \\
04 / 20 / 98 \\
01 / 15 / 98\end{array}$ & 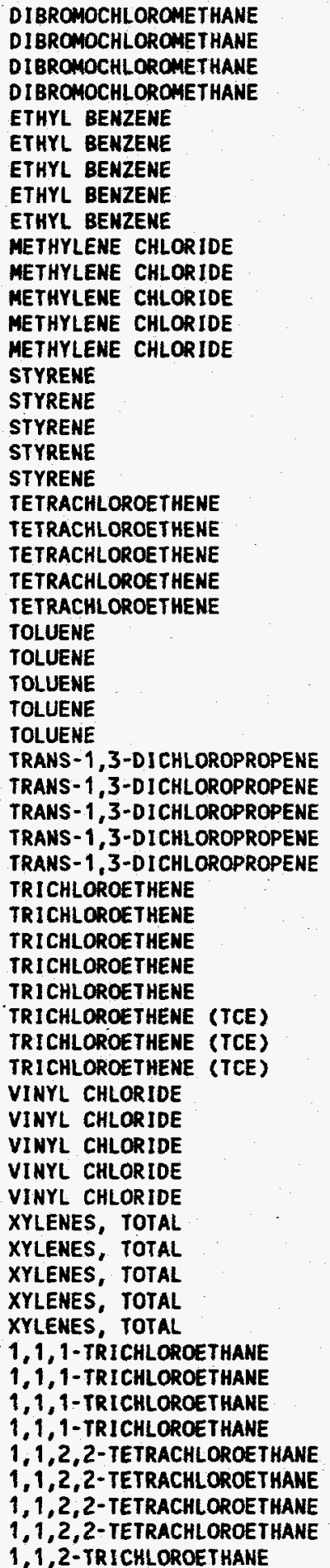 & 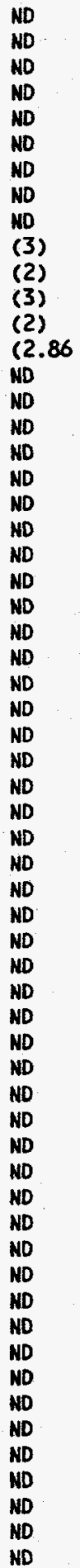 & $\begin{array}{l}10 \\
10 \\
10 \\
10.0 \\
10 \\
10 \\
10 \\
10 \\
10.0 \\
10 \\
10 \\
10 \\
10 \\
10.0 \\
10 \\
10 \\
10 \\
10 \\
10.0 \\
10 \\
10 \\
10 \\
10 \\
10.0 \\
10 \\
10 \\
10 \\
10 \\
10.0 \\
10 \\
10 \\
10 \\
10 \\
10.0 \\
10 \\
10 \\
10 \\
10 \\
10.0 \\
1 \\
1 \\
1.0 \\
10 \\
10 \\
10 \\
10 \\
10.0 \\
10 \\
10 \\
10 \\
10 \\
10.0 \\
10 \\
10 \\
10 \\
10.0 \\
10 \\
10 \\
10 \\
10.0 \\
10\end{array}$ & $\begin{array}{l}U G / L \\
U G / L \\
U G / L \\
U G / L \\
U G / L \\
U G / L \\
U G / L \\
U G / L \\
U G / L \\
U G / L \\
U G / L \\
U G / L \\
U G / L \\
U G / L \\
U G / L \\
U G / L \\
U G / L \\
U G / L \\
U G / L \\
U G / L \\
U G / L \\
U G / L \\
U G / L \\
U G / L \\
U G / L \\
U G / L \\
U G / L \\
U G / L \\
U G / L \\
U G / L \\
U G / L \\
U G / L \\
U G / L \\
U G / L \\
U G / L \\
U G / L \\
U G / L \\
U G / L \\
U G / L \\
U G / L \\
U G / L \\
U G / L \\
U G / L \\
U G / L \\
U G / L \\
U G / L \\
U G / L \\
U G / L \\
U G / L \\
U G / L \\
U G / L \\
U G / L \\
U G / L \\
U G / L \\
U G / L \\
U G / L \\
U G / L \\
U G / L \\
U G / L \\
U G / L \\
U G / L\end{array}$ & $\begin{array}{l}\text { EPA CLP } \\
\text { EPA CLP } \\
\text { EPA CLP } \\
\text { EPA CLP } \\
\text { EPA CLP } \\
\text { EPA CLP } \\
\text { EPA CLP } \\
\text { EPA CLP } \\
\text { EPA CLP } \\
\text { EPA CLP } \\
\text { EPA CLP } \\
\text { EPA CLP } \\
\text { EPA CLP } \\
\text { EPA CLP } \\
\text { EPA CLP } \\
\text { EPA CLP } \\
\text { EPA CLP } \\
\text { EPA CLP } \\
\text { EPA CLP } \\
\text { EPA CLP } \\
\text { EPA CLP } \\
\text { EPA CLP } \\
\text { EPA CLP } \\
\text { EPA CLP } \\
\text { EPA CLP } \\
\text { EPA CLP } \\
\text { EPA CLP } \\
\text { EPA CLP } \\
\text { EPA CLP } \\
\text { EPA CLP } \\
\text { EPA CLP } \\
\text { EPA CLP } \\
\text { EPA CLP } \\
\text { EPA CLP } \\
\text { EPA CLP } \\
\text { EPA CLP } \\
\text { EPA CLP } \\
\text { EPA CLP } \\
\text { EPA CLP } \\
\text { EPA } 8260 \\
\text { EPA } 8260 \\
\text { EPA } 8260 \\
\text { EPA CLP } \\
\text { EPA CLP } \\
\text { EPA CLP } \\
\text { EPA CLP } \\
\text { EPA CLP } \\
\text { EPA CLP } \\
\text { EPA CLP } \\
\text { EPA CLP } \\
\text { EPA CLP } \\
\text { EPA CLP } \\
\text { EPA CLP } C L P \\
\text { EPA CLP } \\
\text { EPA CLP } \\
\text { EPA CLP } \\
\text { EPA CLP } \\
\text { EPA CLP } \\
\text { EPA CLP }\end{array}$ \\
\hline
\end{tabular}




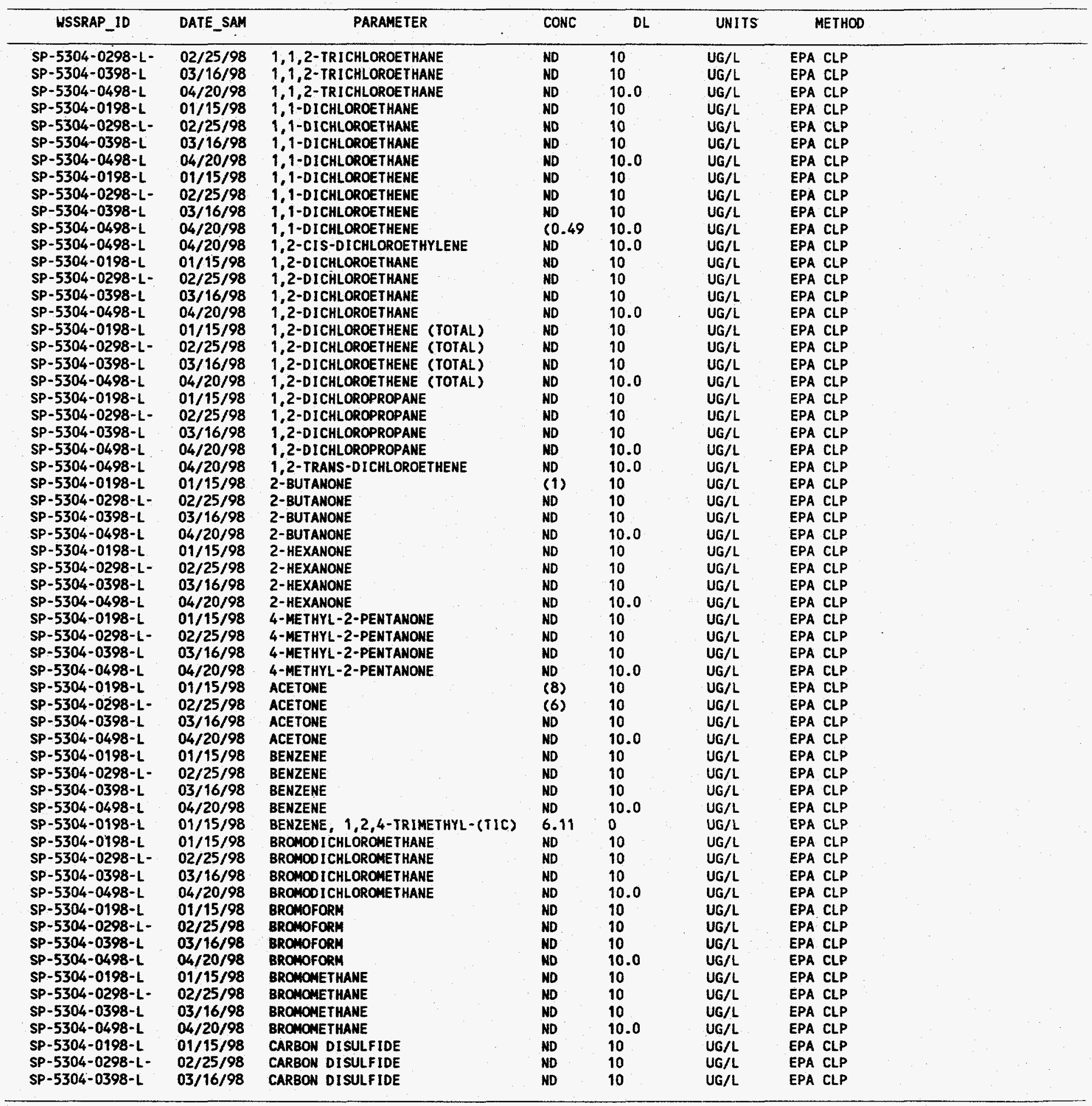




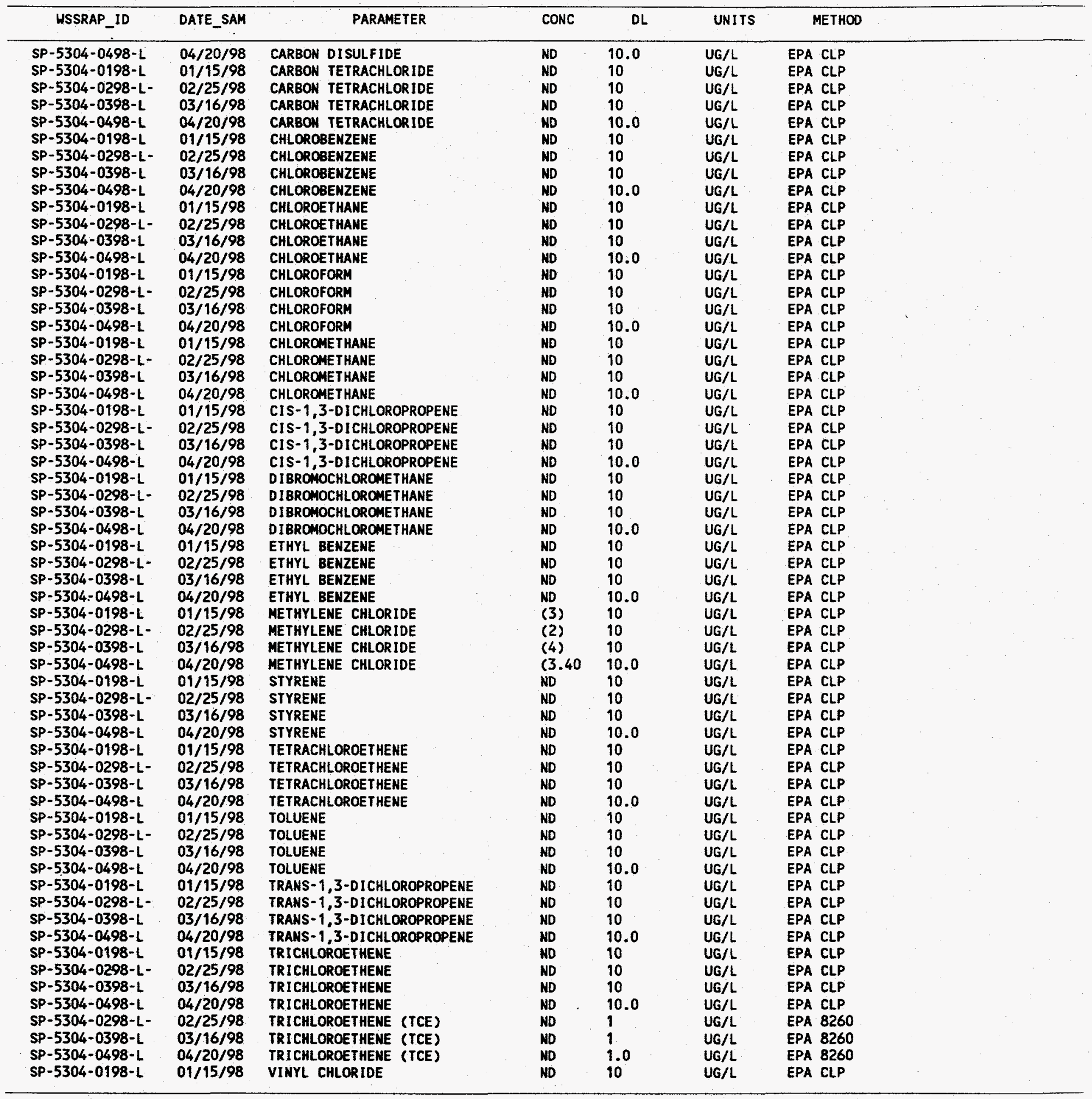




\begin{tabular}{|c|c|c|c|c|c|c|}
\hline WSSRAP_ID & DATE_SAM & PARAMETER & CONC & $\mathrm{DL}$ & UNITS & METHOD \\
\hline 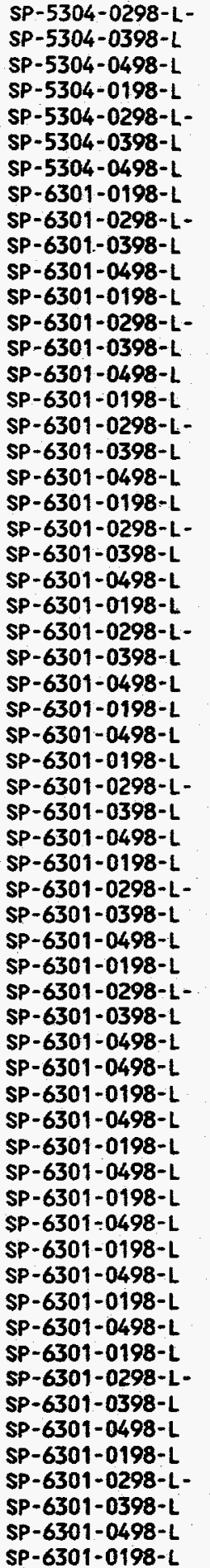 & $\begin{array}{l}02 / 25 / 98 \\
03 / 16 / 98 \\
04 / 20 / 98 \\
01 / 15 / 98 \\
02 / 25 / 98 \\
03 / 16 / 98 \\
04 / 20 / 98 \\
01 / 15 / 98 \\
02 / 25 / 98 \\
03 / 16 / 98 \\
04 / 20 / 98 \\
01 / 15 / 98 \\
02 / 25 / 98 \\
03 / 16 / 98 \\
04 / 20 / 98 \\
01 / 15 / 98 \\
02 / 25 / 98 \\
03 / 16 / 98 \\
04 / 20 / 98 \\
01 / 15 / 98 \\
02 / 25 / 98 \\
03 / 16 / 98 \\
04 / 20 / 98 \\
01 / 15 / 98 \\
02 / 25 / 98 \\
03 / 16 / 98 \\
04 / 20 / 98 \\
01 / 15 / 98 \\
04 / 20 / 98 \\
01 / 15 / 98 \\
02 / 25 / 98 \\
03 / 16 / 98 \\
04 / 20 / 98 \\
01 / 15 / 98 \\
02 / 25 / 98 \\
03 / 16 / 98 \\
04 / 20 / 98 \\
01 / 15 / 98 \\
02 / 25 / 98 \\
03 / 16 / 98 \\
04 / 20 / 98 \\
04 / 20 / 98 \\
01 / 15 / 98 \\
04 / 20 / 98 \\
01 / 15 / 98 \\
04 / 20 / 98 \\
01 / 15 / 98 \\
04 / 20 / 98 \\
01 / 15 / 98 \\
04 / 20 / 98 \\
01 / 15 / 98 \\
04 / 20 / 98 \\
01 / 15 / 98 \\
02 / 25 / 98 \\
03 / 16 / 98 \\
04 / 20 / 98 \\
01 / 15 / 98 \\
02 / 25 / 98 \\
03 / 16 / 98 \\
04 / 20 / 98 \\
01 / 15 / 98\end{array}$ & 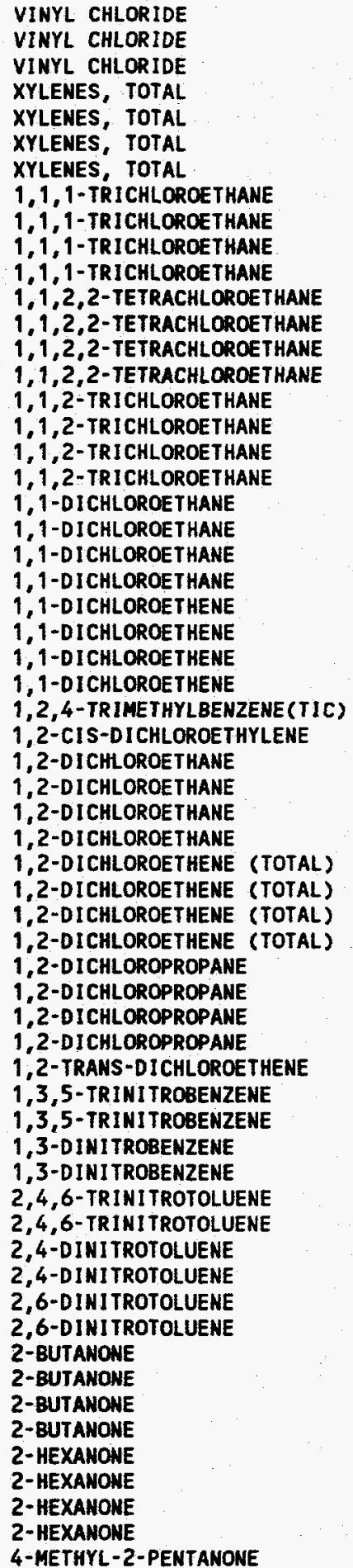 & 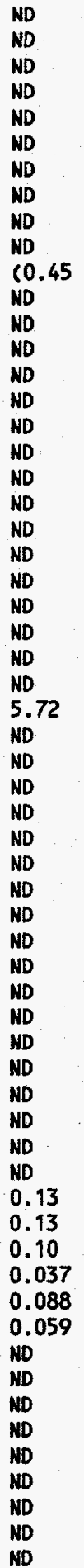 & $\begin{array}{l}10 \\
10 \\
10.0 \\
10 \\
10 \\
10 \\
10.0 \\
10 \\
10 \\
10 \\
10.0 \\
10 \\
10 \\
10 \\
10.0 \\
10 \\
10 \\
10 \\
10.0 \\
10 \\
10 \\
10 \\
10.0 \\
10 \\
10 \\
10 \\
10.0 \\
0 \\
10.0 \\
10 \\
10 \\
10 \\
10.0 \\
10 \\
10 \\
10 \\
10.0 \\
10 \\
10 \\
10 \\
10.0 \\
10.0 \\
0.030 \\
0.030 \\
0.090 \\
0.090 \\
0.030 \\
0.030 \\
0.030 \\
0.030 \\
0.010 \\
0.010 \\
10 \\
10 \\
10 \\
10.0 \\
10 \\
10 \\
10 \\
10.0 \\
10\end{array}$ & $\begin{array}{l}U G / L \\
U G / L \\
U G / L \\
U G / L \\
U G / L \\
U G / L \\
U G / L \\
U G / L \\
U G / L \\
U G / L \\
U G / L \\
U G / L \\
U G / L \\
U G / L \\
U G / L \\
U G / L \\
U G / L \\
U G / L \\
U G / L \\
U G / L \\
U G / L \\
U G / L \\
U G / L \\
U G / L \\
U G / L \\
U G / L \\
U G / L \\
U G / L \\
U G / L \\
U G / L \\
U G / L \\
U G / L \\
U G / L \\
U G / L \\
U G / L \\
U G / L \\
U G / L \\
U G / L \\
U G / L \\
U G / L \\
U G / L \\
U G / L \\
U G / L \\
U G / L \\
U G / L \\
U G / L \\
U G / L \\
U G / L \\
U G / L \\
U G / L \\
U G / L \\
U G / L \\
U G / L \\
U G / L \\
U G / L \\
U G / L \\
U G / L \\
U G / L \\
U G / L \\
U G / L \\
U G / L \\
\text { U. } \\
U\end{array}$ & $\begin{array}{l}\text { EPA CLP } \\
\text { EPA CLP } \\
\text { EPA CLP } \\
\text { EPA CLP } \\
\text { EPA CLP } \\
\text { EPA CLP } \\
\text { EPA CLP } \\
\text { EPA CLP } \\
\text { EPA CLP } \\
\text { EPA CLP } \\
\text { EPA CLP } \\
\text { EPA CLP } \\
\text { EPA CLP } \\
\text { EPA CLP } \\
\text { EPA CLP } \\
\text { EPA CLP } \\
\text { EPA CLP } \\
\text { EPA CLP } \\
\text { EPA CLP } \\
\text { EPA CLP } \\
\text { EPA CLP } \\
\text { EPA CLP } \\
\text { EPA CLP } \\
\text { EPA CLP } \\
\text { EPA CLP } \\
\text { EPA CLP } \\
\text { EPA CLP } \\
\text { EPA CLP } \\
\text { EPA CLP } \\
\text { EPA CLP } \\
\text { EPA CLP } \\
\text { EPA CLP } \\
\text { EPA CLP } \\
\text { EPA CLP } \\
\text { EPA CLP } \\
\text { EPA CLP } \\
\text { EPA CLP } \\
\text { EPA CLP } \\
\text { EPA CLP } \\
\text { EPA CLP } \\
\text { EPA CLP } \\
\text { EPA CLP } \\
\text { USATHAMA } \\
\text { USATHAMA } \\
\text { USATHAMA } \\
\text { USATHAMA } \\
\text { USATHAMA } \\
\text { USATHAMA } \\
\text { USATHAMA } \\
\text { USATHAMA } \\
\text { USATHAMA } \\
\text { USATHAMA } \\
\text { EPA CLP } \\
\text { EPA CLP } \\
\text { EPA CLP } \\
\text { EPA CLP } \\
\text { EPA CLP } \\
\text { EPA CLP } \\
\text { EPA }\end{array}$ \\
\hline
\end{tabular}




\begin{tabular}{|c|c|c|c|c|c|c|}
\hline WSSRAP_ID & DATE_SAM & PARAMETER & CONC & DL. & UNITS & METHOD \\
\hline 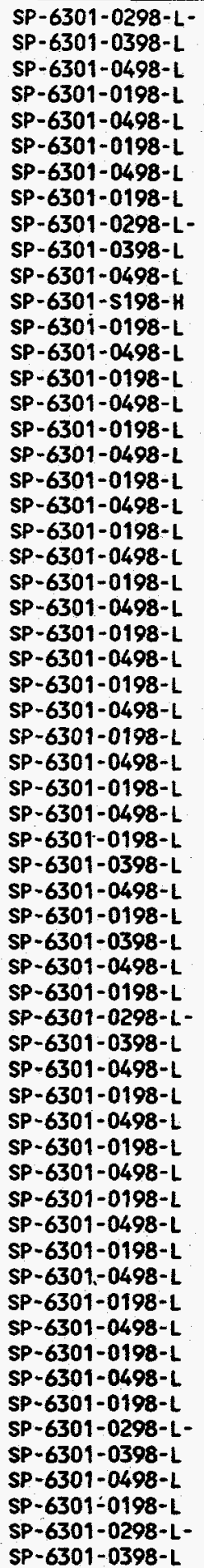 & $\begin{array}{l}02 / 25 / 98 \\
03 / 16 / 98 \\
04 / 20 / 98 \\
01 / 15 / 98 \\
04 / 20 / 98 \\
01 / 15 / 98 \\
04 / 20 / 98 \\
01 / 15 / 98 \\
02 / 25 / 98 \\
03 / 16 / 98 \\
04 / 20 / 98 \\
02 / 11 / 98 \\
01 / 15 / 98 \\
04 / 20 / 98 \\
01 / 15 / 98 \\
04 / 20 / 98 \\
01 / 15 / 98 \\
04 / 20 / 98 \\
01 / 15 / 98 \\
04 / 20 / 98 \\
01 / 15 / 98 \\
04 / 20 / 98 \\
01 / 15 / 98 \\
04 / 20 / 98 \\
01 / 15 / 98 \\
04 / 20 / 98 \\
01 / 15 / 98 \\
04 / 20 / 98 \\
01 / 15 / 98 \\
04 / 20 / 98 \\
01 / 15 / 98 \\
04 / 20 / 98 \\
01 / 15 / 98 \\
03 / 16 / 98 \\
04 / 20 / 98 \\
01 / 15 / 98 \\
03 / 16 / 98 \\
04 / 20 / 98 \\
01 / 15 / 98 \\
02 / 25 / 98 \\
03 / 16 / 98 \\
04 / 20 / 98 \\
01 / 15 / 98 \\
04 / 20 / 98 \\
01 / 15 / 98 \\
04 / 20 / 98 \\
01 / 15 / 98 \\
04 / 20 / 98 \\
01 / 15 / 98 \\
04 / 20 / 98 \\
01 / 15 / 98 \\
04 / 20 / 98 \\
01 / 15 / 98 \\
04 / 20 / 98 \\
01 / 15 / 98 \\
02 / 25 / 98 \\
03 / 16 / 98 \\
04 / 20 / 98 \\
01 / 15 / 98 \\
02 / 25 / 98 \\
03 / 16 / 98\end{array}$ & 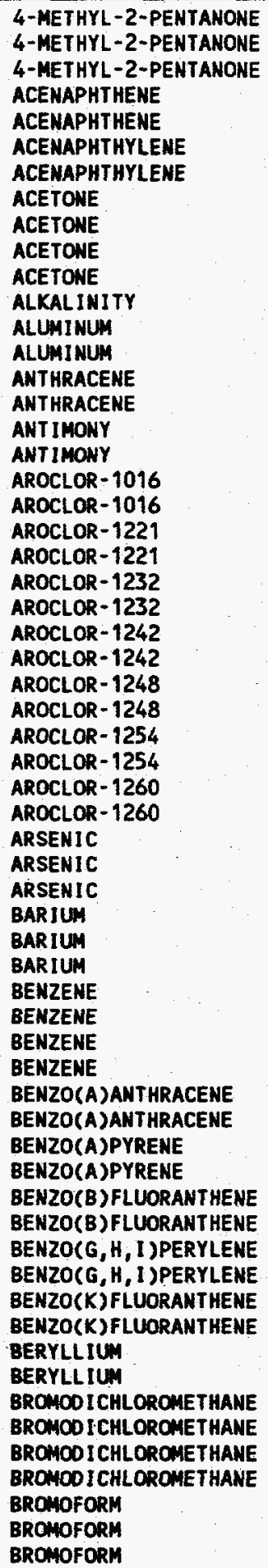 & $\begin{array}{l}\text { ND } \\
\text { ND } \\
\text { ND } \\
\text { ND } \\
\text { ND } \\
(4) \\
\text { (8) } \\
\text { ND } \\
11.2 \\
145 \\
975 \\
1160 \\
\text { ND } \\
\text { ND } \\
\text { ND } \\
\text { ND } \\
\text { ND } \\
\text { ND } \\
\text { ND } \\
\text { ND } \\
\text { ND } \\
\text { ND } \\
\text { ND } \\
\text { ND } \\
\text { ND } \\
\text { ND } \\
\text { ND } \\
\text { ND } \\
\text { ND } \\
\text { ND } \\
\text { ND } \\
\text { ND } \\
\text { ND } \\
93.9 \\
85.2 \\
89.1 \\
\text { ND } \\
\text { ND } \\
\text { ND } \\
\text { ND } \\
\text { ND } \\
\text { ND } \\
\text { ND } \\
\text { ND } \\
\text { ND } \\
\text { ND } \\
\text { ND } \\
\text { ND } \\
\text { ND } \\
\text { ND } \\
\text { ND } \\
\text { ND } \\
\text { ND } \\
\text { ND } \\
\text { No }\end{array}$ & $\begin{array}{l}10 \\
10 \\
10.0 \\
5.0 \\
1.0 \\
5.0 \\
1.0 \\
10 \\
10 \\
10 \\
10.0 \\
5.00 \\
31.3 \\
12.4 \\
5.0 \\
1.0 \\
42.5 \\
2.5 \\
1.0 \\
0.10 \\
1.0 \\
0.10 \\
1.0 \\
0.10 \\
1.0 \\
0.10 \\
1.0 \\
0.10 \\
1.0 \\
0.10 \\
1.0 \\
0.10 \\
1.3 \\
1.9 \\
5.3 \\
0.40 \\
1.0 \\
0.3 \\
10 \\
10 \\
10 \\
10.0 \\
5.0 \\
1.0 \\
5.0 \\
1.0 \\
5.0 \\
1.0 \\
5.0 \\
1.0 \\
5.0 \\
1.0 \\
0.40 \\
0.2 \\
10 \\
10 \\
10 \\
10.0 \\
10 \\
10 \\
10 \\
\end{array}$ & $\begin{array}{l}U G / L \\
U G / L \\
U G / L \\
U G / L \\
U G / L \\
U G / L \\
U G / L \\
U G / L \\
U G / L \\
U G / L \\
U G / L \\
U G / L \\
U G / L \\
U G / L \\
U G / L \\
U G / L \\
U G / L \\
U G / L \\
U G / L \\
U G / L \\
U G / L \\
U G / L \\
U G / L \\
U G / L \\
U G / L \\
U G / L \\
U G / L \\
U G / L \\
U G / L \\
U G / L \\
U G / L \\
U G / L \\
U G / L \\
U G / L \\
U G / L \\
U G / L \\
U G / L \\
U G / L \\
U G / L \\
U G / L \\
U G / L \\
U G / L \\
U G / L \\
U G / L \\
U G / L \\
U G / L \\
U G / L \\
U G / L \\
U G / L \\
U G / L \\
U G / L \\
U G / L \\
U G / L \\
U G / L \\
U G / L \\
U G / L \\
U G / L \\
U G / \\
U\end{array}$ & 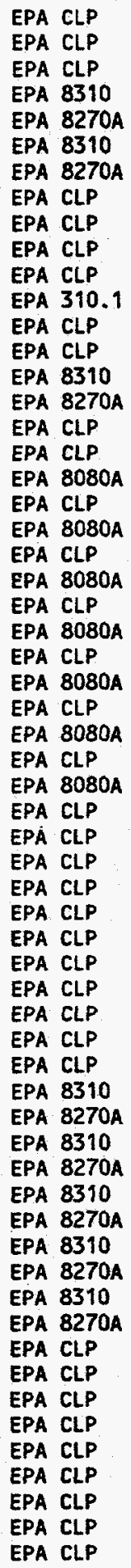 \\
\hline
\end{tabular}




\begin{tabular}{|c|c|c|c|c|c|c|}
\hline WSSRAP_ID & DATE_SAM & PARAMETER & CONC & $D L$ & UNITS & METHOD \\
\hline 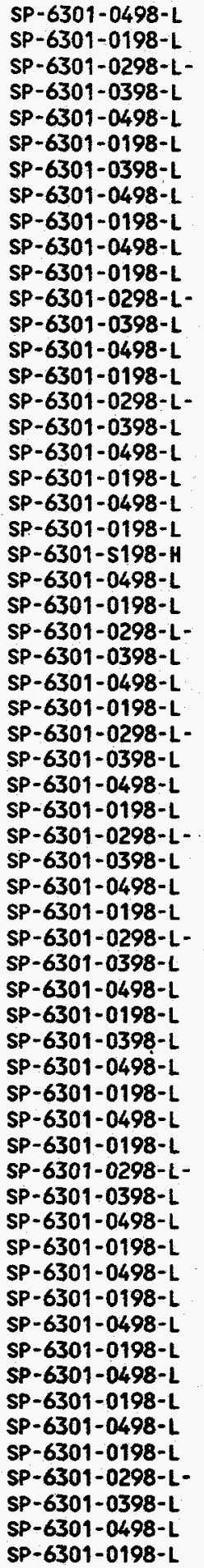 & $\begin{array}{l}04 / 20 / 98 \\
01 / 15 / 98 \\
02 / 25 / 98 \\
03 / 16 / 98 \\
04 / 20 / 98 \\
01 / 15 / 98 \\
03 / 16 / 98 \\
04 / 20 / 98 \\
01 / 15 / 98 \\
04 / 20 / 98 \\
01 / 15 / 98 \\
02 / 25 / 98 \\
03 / 16 / 98 \\
04 / 20 / 98 \\
01 / 15 / 98 \\
02 / 25 / 98 \\
03 / 16 / 98 \\
04 / 20 / 98 \\
01 / 15 / 98 \\
04 / 20 / 98 \\
01 / 15 / 98 \\
02 / 11 / 98 \\
04 / 20 / 98 \\
01 / 15 / 98 \\
02 / 25 / 98 \\
03 / 16 / 98 \\
04 / 20 / 98 \\
01 / 15 / 98 \\
02 / 25 / 98 \\
03 / 16 / 98 \\
04 / 20 / 98 \\
01 / 15 / 98 \\
02 / 25 / 98 \\
03 / 16 / 98 \\
04 / 20 / 98 \\
01 / 15 / 98 \\
02 / 25 / 98 \\
03 / 16 / 98 \\
04 / 20 / 98 \\
01 / 15 / 98 \\
03 / 16 / 98 \\
04 / 20 / 98 \\
01 / 15 / 98 \\
04 / 20 / 98 \\
01 / 15 / 98 \\
02 / 25 / 98 \\
03 / 16 / 98 \\
04 / 20 / 98 \\
01 / 15 / 98 \\
04 / 20 / 98 \\
01 / 15 / 98 \\
04 / 20 / 98 \\
01 / 15 / 98 \\
04 / 20 / 98 \\
01 / 15 / 98 \\
04 / 20 / 98 \\
01 / 15 / 98 \\
02 / 25 / 98 \\
03 / 16 / 98 \\
04 / 20 / 98 \\
01 / 15 / 98\end{array}$ & 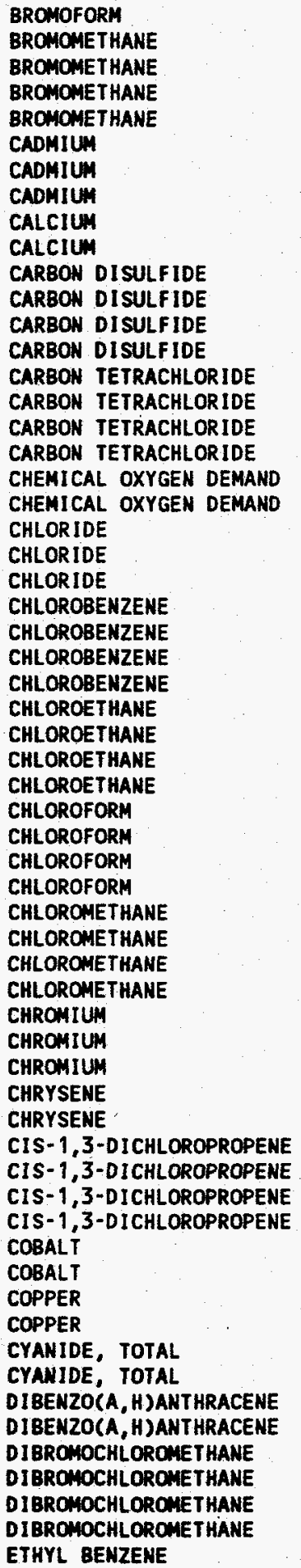 & 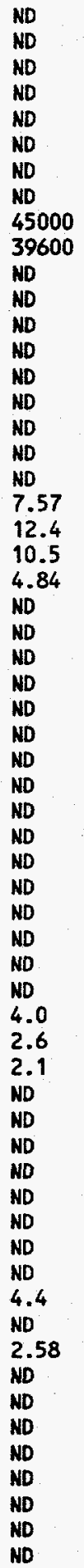 & 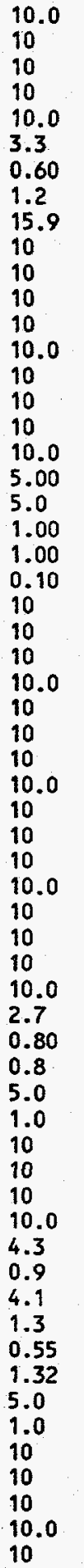 & $\begin{array}{l}U G / L \\
U G / L \\
U G / L \\
U G / L \\
U G / L \\
U G / L \\
U G / L \\
U G / L \\
U G / L \\
U G / L \\
U G / L \\
U G / L \\
U G / L \\
U G / L \\
U G / L \\
U G / L \\
U G / L \\
U G / L \\
M G / L \\
M G / L \\
M G / L \\
U G / L \\
U G / L \\
U G / L \\
U G / L \\
U G / L \\
U G / L \\
U G / L \\
U G / L \\
U G / L \\
U G / L \\
U G / L \\
U G / L \\
U G / L \\
U G / L \\
U G / L \\
U G / L \\
U G / L \\
U G / L \\
U G / L \\
U G / L \\
U G / L \\
U G / L \\
U G / L \\
U G / L \\
U G / L \\
U G / L \\
U G / L \\
U G / L \\
U G / L \\
U G / L \\
U G / L \\
U G / L \\
U G / L \\
U G / L \\
U G / L \\
U G / L \\
U G / L \\
U G / L \\
U G / L \\
U G / L \\
\text { U. } \\
U\end{array}$ & $\begin{array}{l}\text { EPA CLP } \\
\text { EPA CLP } \\
\text { EPA CLP } \\
\text { EPA CLP } \\
\text { EPA CLP } \\
\text { EPA CLP } \\
\text { EPA CLP } \\
\text { EPA CLP } \\
\text { EPA CLP } \\
\text { EPA CLP } \\
\text { EPA CLP } \\
\text { EPA CLP } \\
\text { EPA CLP } \\
\text { EPA CLP } \\
\text { EPA CLP } \\
\text { EPA CLP } \\
\text { EPA CLP } \\
\text { EPA CLP } \\
\text { EPA } 410.4 \\
\text { EPA } 410.4 \\
\text { EPA } 300.0 \\
\text { EPA } 300.0 \\
\text { EPA } 300.0 \\
\text { EPA CLP } \\
\text { EPA CLP } \\
\text { EPA CLP } \\
\text { EPA CLP } \\
\text { EPA CLP } \\
\text { EPA CLP } \\
\text { EPA CLP } \\
\text { EPA CLP } \\
\text { EPA CLP } \\
\text { EPA CLP } \\
\text { EPA CLP } \\
\text { EPA CLP } \\
\text { EPA CLP } \\
\text { EPA CLP } \\
\text { EPA CLP } \\
\text { EPA CLP } \\
\text { EPA CLP } \\
\text { EPA CLP } \\
\text { EPA CLP } \\
\text { EPA } 8310 \\
\text { EPA } 8270 A \\
\text { EPA CLP } \\
\text { EPA CLP } \\
\text { EPA CLP } \\
\text { EPA CLP } \\
\text { EPA CLP } \\
\text { EPA CLP } \\
\text { EPA CLP } \\
\text { EPA CLP } \\
\text { EPA CLP } \\
\text { EPA CLP } \\
\text { EPA } 8310 \\
\text { EPA } 8270 A \\
\text { EPA CLP } \\
\text { EPA CLP } \\
\text { EPA CLP } \\
\text { EPA CLP } \\
\text { EPA CLPP }\end{array}$ \\
\hline
\end{tabular}




\begin{tabular}{|c|c|c|c|c|c|c|}
\hline WSSRAP_ID & DATE_SAM & PARAMETER & CONC & $D L$ & UNITS & METHOD \\
\hline 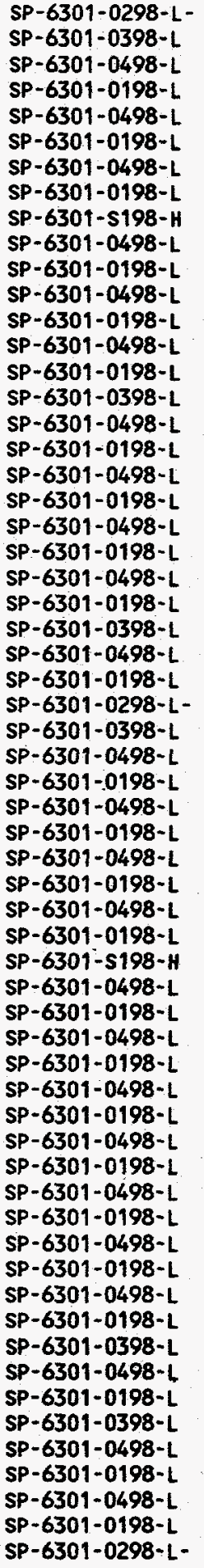 & $\begin{array}{l}02 / 25 / 98 \\
03 / 16 / 98 \\
04 / 20 / 98 \\
01 / 15 / 98 \\
04 / 20 / 98 \\
01 / 15 / 98 \\
04 / 20 / 98 \\
01 / 15 / 98 \\
02 / 11 / 98 \\
04 / 20 / 98 \\
01 / 15 / 98 \\
04 / 20 / 98 \\
01 / 15 / 98 \\
04 / 20 / 98 \\
01 / 15 / 98 \\
03 / 16 / 98 \\
04 / 20 / 98 \\
01 / 15 / 98 \\
04 / 20 / 98 \\
01 / 15 / 98 \\
04 / 20 / 98 \\
01 / 15 / 98 \\
04 / 20 / 98 \\
01 / 15 / 98 \\
03 / 16 / 98 \\
04 / 20 / 98 \\
01 / 15 / 98 \\
02 / 25 / 98 \\
03 / 16 / 98 \\
04 / 20 / 98 \\
01 / 15 / 98 \\
04 / 20 / 98 \\
01 / 15 / 98 \\
04 / 20 / 98 \\
01 / 15 / 98 \\
04 / 20 / 98 \\
01 / 15 / 98 \\
02 / 11 / 98 \\
04 / 20 / 98 \\
01 / 15 / 98 \\
04 / 20 / 98 \\
01 / 15 / 98 \\
04 / 20 / 98 \\
01 / 15 / 98 \\
04 / 20 / 98 \\
01 / 15 / 98 \\
04 / 20 / 98 \\
01 / 15 / 98 \\
04 / 20 / 98 \\
01 / 15 / 98 \\
04 / 20 / 98 \\
01 / 15 / 98 \\
03 / 16 / 98 \\
04 / 20 / 98 \\
01 / 15 / 98 \\
03 / 16 / 98 \\
04 / 20 / 98 \\
01 / 15 / 98 \\
04 / 20 / 98 \\
01 / 15 / 98 \\
02 / 25 / 98\end{array}$ & $\begin{array}{l}\text { ETHYL BENZENE } \\
\text { ETHYL BENZENE } \\
\text { ETHYL BENZENE } \\
\text { FLUORANTHENE } \\
\text { FLUORANTHENE } \\
\text { FLUORENE } \\
\text { FLUORENE } \\
\text { FLUORIDE } \\
\text { FLUORIDE } \\
\text { FLUORIDE } \\
\text { INDENO(1,2,3-CD)PYRENE } \\
\text { INDENO(1,2,3-CD)PYRENE } \\
\text { IRON } \\
\text { IRON } \\
\text { LEAD } \\
\text { LEAD } \\
\text { LEAD } \\
\text { LITHIUM } \\
\text { LITHIUM } \\
\text { MAGNESIUM } \\
\text { MAGNESIUM } \\
\text { MANGANESE } \\
\text { MANGANESE } \\
\text { MERCURY } \\
\text { MERCURY } \\
\text { MERCURY } \\
\text { METHYLENE CHLORIDE } \\
\text { METHYLNE CHLORIDE } \\
\text { METHYLENE CHLORIDE } \\
\text { METHYLENE CHLORIDE } \\
\text { MOLYBDENUM } \\
\text { MOLYBDENUM } \\
\text { NAPHTHALENE } \\
\text { NAPHTHALENE } \\
\text { HICKEL } \\
\text { NICKEL } \\
\text { NITRATE-N } \\
\text { NITRATE-N } \\
\text { NITRATE-N } \\
\text { NITROBENZENE } \\
\text { HITROBENZENE } \\
\text { PHENANTHRENE } \\
\text { PHENANTHRENE } \\
\text { POTASSIUM } \\
\text { POTASSIUM } \\
\text { PYRENE } \\
\text { PYRENE } \\
\text { RADIUM-226 } \\
\text { RADIUM-226 } \\
\text { RADIUM-228 } \\
\text { RADIUM-228 } \\
\text { SELENIUM } \\
\text { SELENIUM } \\
\text { SELENIUM } \\
\text { SILVER } \\
\text { SILVER } \\
\text { SILVER } \\
\text { SODIUM } \\
\text { SODIUM } \\
\text { STYRENE } \\
\text { STYRENE } \\
\end{array}$ & $\begin{array}{l}\text { ND } \\
\text { ND } \\
\text { ND } \\
\text { ND } \\
\text { ND } \\
\text { ND } \\
0.26 \\
0.21 \\
0.066 \\
\text { ND } \\
\text { ND } \\
858 \\
999 \\
1.0 \\
2.4 \\
\text { ND } \\
5.0 \\
4.3 \\
11300 \\
8170 \\
14.0 \\
17.8 \\
\text { ND } \\
\text { ND } \\
\text { ND } \\
(3) \\
(2) \\
(2) \\
\text { (3.49 } \\
\text { ND } \\
\text { ND } \\
\text { ND } \\
\text { ND } \\
\text { ND } \\
1.9 \\
2.39 \\
5.33 \\
2.64 \\
\text { ND } \\
\text { ND } \\
\text { ND } \\
\text { ND } \\
3180 \\
2410 \\
\text { ND } \\
\text { ND } \\
0.066 \\
0.936 \\
\text { O. } \\
2.66 \\
3.6 \\
\text { ND } \\
\text { ND } \\
\text { ND } \\
\text { ND } \\
\text { ND } \\
20300 \\
9770 \\
\text { ND } \\
\text { ND }\end{array}$ & $\begin{array}{l}10 \\
10 \\
10.0 \\
5.0 \\
1.0 \\
5.0 \\
1.0 \\
0.10 \\
0.10 \\
0.05 \\
5.0 \\
1.0 \\
5.9 \\
2.6 \\
0.90 \\
1.1 \\
2 \\
4.1 \\
0.2 \\
54.5 \\
8.9 \\
1.0 \\
0.4 \\
0.10 \\
0.10 \\
0.1 \\
10 \\
10 \\
10 \\
10.0 \\
11.1 \\
2.1 \\
5.0 \\
1.0 \\
14.6 \\
1.1 \\
0.50 \\
1.00 \\
0.05 \\
0.030 \\
0.030 \\
5.0 \\
1.0 \\
2180 \\
2.1 \\
10 \\
5.0 \\
10 \\
1.0 \\
0.023 \\
0.348 \\
0.325 \\
0.562 \\
2.2 \\
3.1 \\
2.5 \\
6.0 \\
0.70 \\
\end{array}$ & $\begin{array}{l}U G / L \\
U G / L \\
U G / L \\
U G / L \\
U G / L \\
U G / L \\
U G / L \\
M G / L \\
M G / L \\
M G / L \\
U G / L \\
U G / L \\
U G / L \\
U G / L \\
U G / L \\
U G / L \\
U G / L \\
U G / L \\
U G / L \\
U G / L \\
U G / L \\
U G / L \\
U G / L \\
U G / L \\
U G / L \\
U G / L \\
U G / L \\
U G / L \\
U G / L \\
U G / L \\
U G / L \\
U G / L \\
U G / L \\
U G / L \\
U G / L \\
U G / L \\
M G / L \\
M G / L \\
M G / L \\
U G / L \\
U G / L \\
U G / L \\
U G / L \\
U G / L \\
U G / L \\
U G / L \\
U G / L \\
P C I / L \\
P C I / L \\
P C I / L \\
P C I / L \\
U G / L \\
U G / L \\
U G / L \\
U G / L \\
U G / L \\
U G / L \\
U G / L \\
U G / L \\
U G / L \\
U G / L \\
\end{array}$ & $\begin{array}{l}\text { EPA CLP } \\
\text { EPA CLP } \\
\text { EPA CLP } \\
\text { EPA } 8310 \\
\text { EPA } 8270 A \\
\text { EPA } 8310 \\
\text { EPA } 8270 A \\
\text { EPA } 300.0 \\
\text { EPA } 300.0 \\
\text { EPA } 300.0 \\
\text { EPA } 8310 \\
\text { EPA } 8270 A \\
\text { EPA } \text { CLP } \\
\text { EPA CLP } \\
\text { EPA CLP } \\
\text { EPA CLP } \\
\text { EPA CLP } \\
\text { EPA CLP } \\
\text { EPA CLP } \\
\text { EPA CLP } \\
\text { EPA CLP } \\
\text { EPA CLP } \\
\text { EPA CLP } \\
\text { EPA CLP } \\
\text { EPA CLP } \\
\text { EPA CLP } \\
\text { EPA CLP } \\
\text { EPA CLP } \\
\text { EPA CLP } \\
\text { EPA CLP } \\
\text { EPA CLP } \\
\text { EPA CLP } \\
\text { EPA } 8310 \\
\text { EPA } 8270 A \\
\text { EPA CLP } \\
\text { EPA CLP } \\
\text { EPA } 353.1 \\
\text { EPA } 353.1 \\
\text { EPA } 353.1 \\
\text { USATHAMA } \\
\text { USATHAMA } \\
\text { EPA } 8310 \\
\text { EPA } 8270 A \\
\text { EPA CLP } \\
\text { EPA CLP } \\
\text { EPA } 8310 \\
\text { EPA } 8270 A \\
\text { EPA } 904.0 \\
\text { EPA } 903.1 \\
\text { EPA } 904.0 \\
\text { EPA } 904.0 \\
\text { EPA CLP } \\
\text { EPA CLP } \\
\text { EPA CLP } \\
\text { EPA CLP } \\
\text { EPA CLP } \\
\text { EPA CLP } \\
\text { EPA CLLP } \\
\text { EPA CLP } \\
\text { EPA CLP } \\
\text { EPA CLPP }\end{array}$ \\
\hline
\end{tabular}




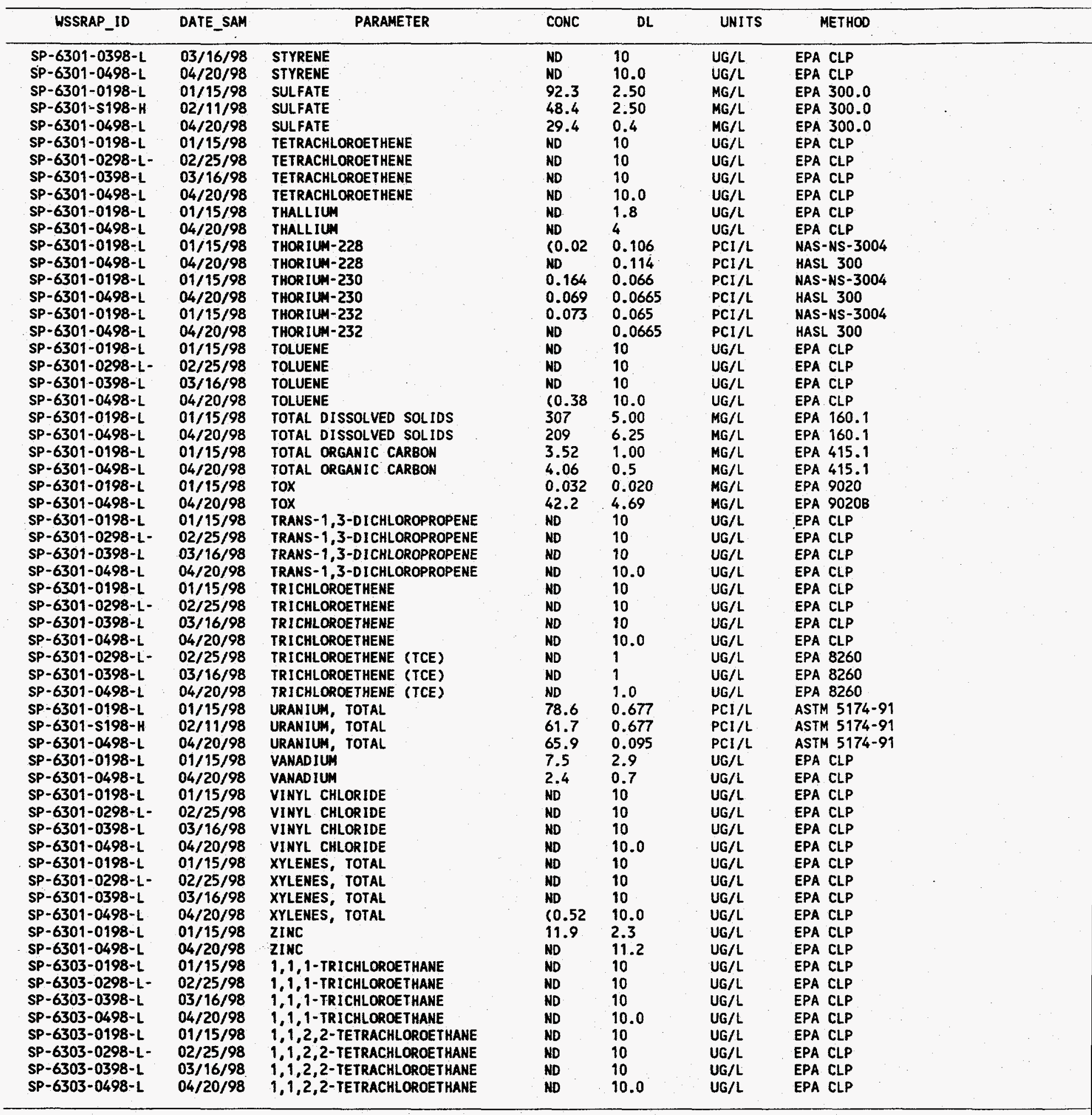




\begin{tabular}{|c|c|c|c|c|c|c|}
\hline WSSRAP_ID & DATE_SAM & PARAMETER & CONC & $\mathrm{DL}$ & UNITS & METHOD \\
\hline 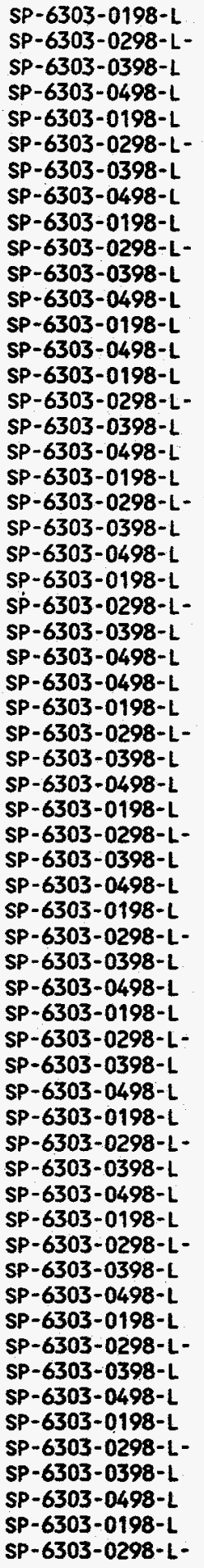 & $\begin{array}{l}01 / 15 / 98 \\
02 / 25 / 98 \\
03 / 16 / 98 \\
04 / 20 / 98 \\
01 / 15 / 98 \\
02 / 25 / 98 \\
03 / 16 / 98 \\
04 / 20 / 98 \\
01 / 15 / 98 \\
02 / 25 / 98 \\
03 / 16 / 98 \\
04 / 20 / 98 \\
01 / 15 / 98 \\
04 / 20 / 98 \\
01 / 15 / 98 \\
02 / 25 / 98 \\
03 / 16 / 98 \\
04 / 20 / 98 \\
01 / 15 / 98 \\
02 / 25 / 98 \\
03 / 16 / 98 \\
04 / 20 / 98 \\
01 / 15 / 98 \\
02 / 25 / 98 \\
03 / 16 / 98 \\
04 / 20 / 98 \\
04 / 20 / 98 \\
01 / 15 / 98 \\
02 / 25 / 98 \\
03 / 16 / 98 \\
04 / 20 / 98 \\
01 / 15 / 98 \\
02 / 25 / 98 \\
03 / 16 / 98 \\
04 / 20 / 98 \\
01 / 15 / 98 \\
02 / 25 / 98 \\
03 / 16 / 98 \\
04 / 20 / 98 \\
01 / 15 / 98 \\
02 / 25 / 98 \\
03 / 16 / 98 \\
04 / 20 / 98 \\
01 / 15 / 98 \\
02 / 25 / 98 \\
03 / 16 / 98 \\
04 / 20 / 98 \\
01 / 15 / 98 \\
02 / 25 / 98 \\
03 / 16 / 98 \\
04 / 20 / 98 \\
01 / 15 / 98 \\
02 / 25 / 98 \\
03 / 16 / 98 \\
04 / 20 / 98 \\
01 / 15 / 98 \\
02 / 25 / 98 \\
03 / 16 / 98 \\
04 / 20 / 98 \\
01 / 15 / 98 \\
02 / 25 / 98\end{array}$ & 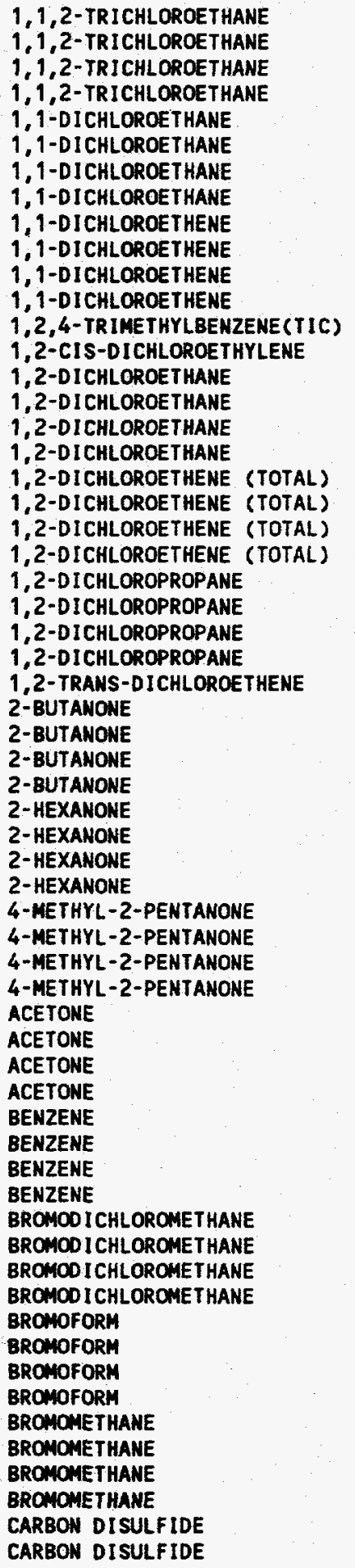 & 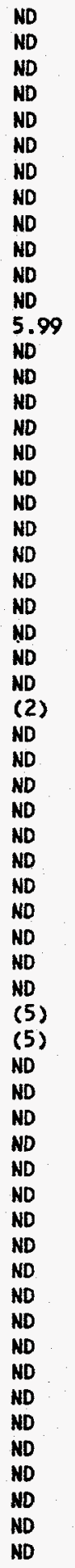 & 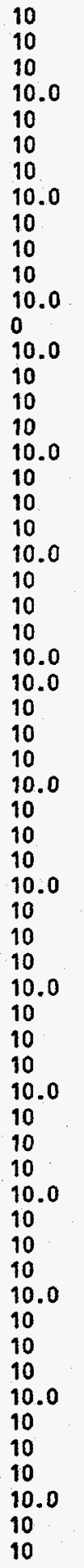 & 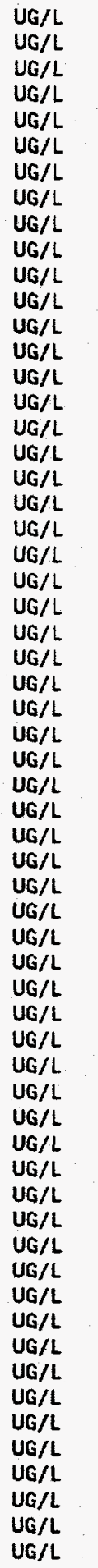 & 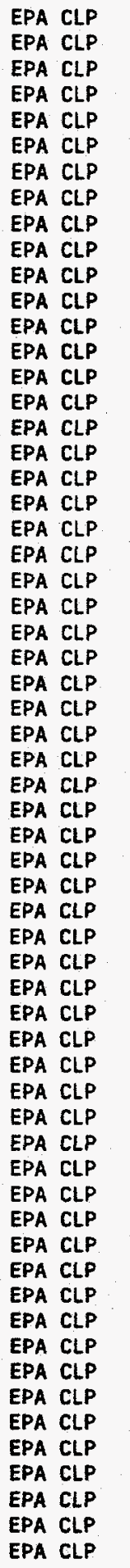 \\
\hline
\end{tabular}




\begin{tabular}{|c|c|c|c|c|c|c|}
\hline WSSRAP_ID & DATE_SAM & PARAMETER & CONC & DL & UNITS & METHOD \\
\hline 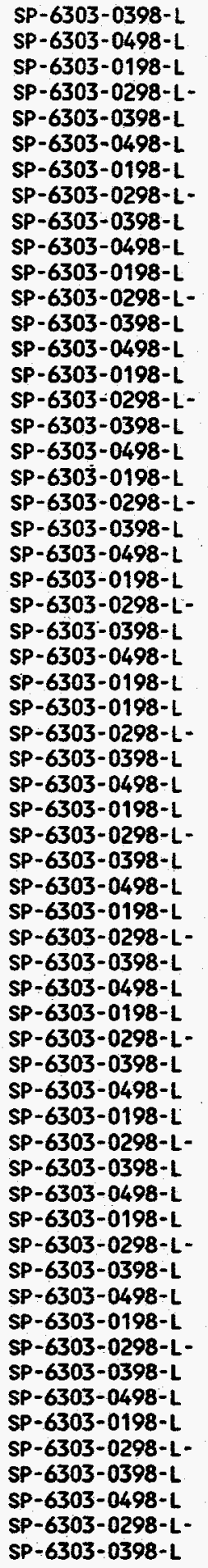 & $\begin{array}{l}03 / 16 / 98 \\
04 / 20 / 98 \\
01 / 15 / 98 \\
02 / 25 / 98 \\
03 / 16 / 98 \\
04 / 20 / 98 \\
01 / 15 / 98 \\
02 / 25 / 98 \\
03 / 16 / 98 \\
04 / 20 / 98 \\
01 / 15 / 98 \\
02 / 25 / 98 \\
03 / 16 / 98 \\
04 / 20 / 98 \\
01 / 15 / 98 \\
02 / 25 / 98 \\
03 / 16 / 98 \\
04 / 20 / 98 \\
01 / 15 / 98 \\
02 / 25 / 98 \\
03 / 16 / 98 \\
04 / 20 / 98 \\
01 / 15 / 98 \\
02 / 25 / 98 \\
03 / 16 / 98 \\
04 / 20 / 98 \\
01 / 15 / 98 \\
01 / 15 / 98 \\
02 / 25 / 98 \\
03 / 16 / 98 \\
04 / 20 / 98 \\
01 / 15 / 98 \\
02 / 25 / 98 \\
03 / 16 / 98 \\
04 / 20 / 98 \\
01 / 15 / 98 \\
02 / 25 / 98 \\
03 / 16 / 98 \\
04 / 20 / 98 \\
01 / 15 / 98 \\
02 / 25 / 98 \\
03 / 16 / 98 \\
04 / 20 / 98 \\
01 / 15 / 98 \\
02 / 25 / 98 \\
03 / 16 / 98 \\
04 / 20 / 98 \\
01 / 15 / 98 \\
02 / 25 / 98 \\
03 / 16 / 98 \\
04 / 20 / 98 \\
01 / 15 / 98 \\
02 / 25 / 98 \\
03 / 16 / 98 \\
04 / 20 / 98 \\
01 / 15 / 98 \\
02 / 25 / 98 \\
03 / 16 / 98 \\
04 / 20 / 98 \\
02 / 25 / 98 \\
03 / 16 / 98\end{array}$ & 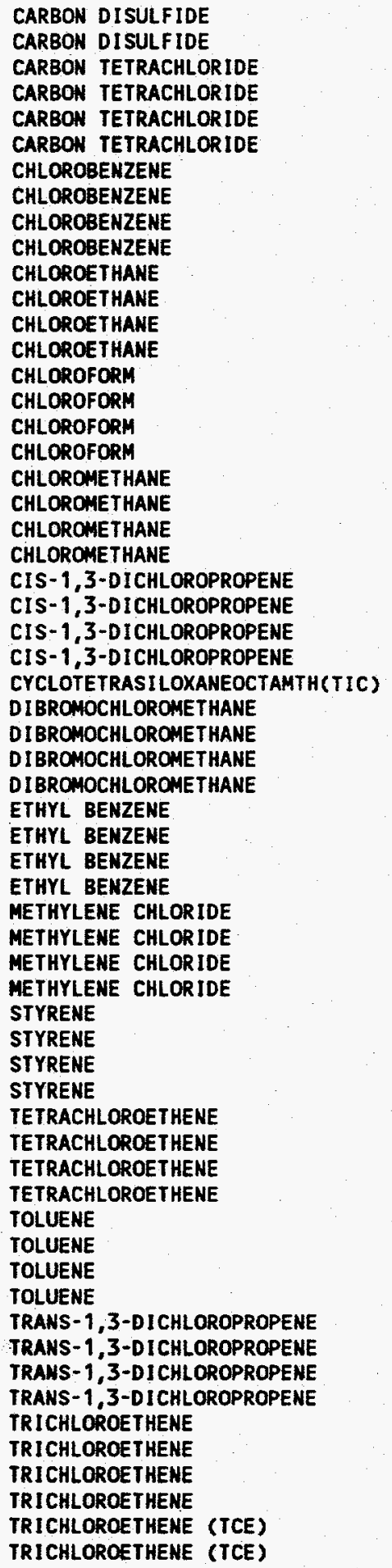 & $\begin{array}{l}\text { ND } \\
\text { ND } \\
\text { ND } \\
\text { ND } \\
\text { ND } \\
\text { ND } \\
\text { ND } \\
\text { ND } \\
\text { ND } \\
\text { ND } \\
\text { ND } \\
\text { ND } \\
\text { ND } \\
\text { ND } \\
\text { ND } \\
\text { ND } \\
\text { ND } \\
\text { ND } \\
\text { ND } \\
\text { ND } \\
\text { ND } \\
\text { ND } \\
\text { ND } \\
\text { ND } \\
\text { ND } \\
\text { ND } \\
9.63 \\
\text { ND } \\
\text { ND } \\
\text { ND } \\
\text { ND } \\
\text { ND } \\
\text { ND } \\
\text { ND } \\
\text { ND } \\
\text { (3) } \\
\text { (2) } \\
\text { (4) } \\
\text { (3.23 } \\
\text { ND } \\
\text { ND } \\
\text { ND } \\
\text { ND } \\
\text { ND } \\
\text { ND } \\
\text { ND } \\
\text { C1.38 } \\
\text { ND } \\
\text { ND } \\
\text { ND } \\
\text { ND } \\
\text { ND } \\
\text { ND } \\
\text { ND } \\
\text { ND } \\
\text { ND } \\
\text { ND } \\
\text { ND } \\
\text { C.48 } \\
\text { CO.3) }\end{array}$ & $\begin{array}{l}10 \\
10.0 \\
10 \\
10 \\
10 \\
10.0 \\
10 \\
10 \\
10 \\
10.0 \\
10 \\
10 \\
10 \\
10.0 \\
10 \\
10 \\
10 \\
10.0 \\
10 \\
10 \\
10 \\
10.0 \\
10 \\
10 \\
10 \\
10.0 \\
0 \\
10 \\
10 \\
10 \\
10.0 \\
10 \\
10 \\
10 \\
10.0 \\
10 \\
10 \\
10 \\
10.0 \\
10 \\
10 \\
10 \\
10.0 \\
10 \\
10 \\
10 \\
10.0 \\
10 \\
10 \\
10 \\
10.0 \\
10 \\
10 \\
10 \\
10.0 \\
10 \\
10 \\
10 \\
10.0 \\
1 \\
1 \\
1 \\
\\
\\
\\
\\
\\
\end{array}$ & $\begin{array}{l}U G / L \\
U G / L \\
U G / L \\
U G / L \\
U G / L \\
U G / L \\
U G / L \\
U G / L \\
U G / L \\
U G / L \\
U G / L \\
U G / L \\
U G / L \\
U G / L \\
U G / L \\
U G / L \\
U G / L \\
U G / L \\
U G / L \\
U G / L \\
U G / L \\
U G / L \\
U G / L \\
U G / L \\
U G / L \\
U G / L \\
U G / L \\
U G / L \\
U G / L \\
U G / L \\
U G / L \\
U G / L \\
U G / L \\
U G / L \\
U G / L \\
U G / L \\
U G / L \\
U G / L \\
U G / L \\
U G / L \\
U G / L \\
U G / L \\
U G / L \\
U G / L \\
U G / L \\
U G / L \\
U G / L \\
U G / L \\
U G / L \\
U G / L \\
U G / L \\
U G / L \\
U G / L \\
U G / L \\
U G / L \\
U G / L \\
U G / L \\
U G / L \\
U G / L \\
U G / L \\
U G / L\end{array}$ & $\begin{array}{l}\text { EPA CLP } \\
\text { EPA CLP } \\
\text { EPA CLP } \\
\text { EPA CLP } \\
\text { EPA CLP } \\
\text { EPA CLP } \\
\text { EPA CLP } \\
\text { EPA CLP } \\
\text { EPA CLP } \\
\text { EPA CLP } \\
\text { EPA CLP } \\
\text { EPA CLP } \\
\text { EPA CLP } \\
\text { EPA CLP } \\
\text { EPA CLP } \\
\text { EPA CLP } \\
\text { EPA CLP } \\
\text { EPA CLP } \\
\text { EPA CLP } \\
\text { EPA CLP } \\
\text { EPA CLP } \\
\text { EPA CLP } \\
\text { EPA CLP } \\
\text { EPA CLP } \\
\text { EPA CLP } \\
\text { EPA CLP } \\
\text { EPA CLP } \\
\text { EPA CLP } \\
\text { EPA CLP } \\
\text { EPA CLP } \\
\text { EPA CLP } \\
\text { EPA CLP } \\
\text { EPA CLP } \\
\text { EPA CLP } \\
\text { EPA CLP } \\
\text { EPA CLP } \\
\text { EPA CLP } \\
\text { EPA CLP } \\
\text { EPA CLP } \\
\text { EPA CLP } \\
\text { EPA CLP } \\
\text { EPA CLP } \\
\text { EPA CLP } \\
\text { EPA CLP } \\
\text { EPA CLP } \\
\text { EPA CLP } \\
\text { EPA CLP } \\
\text { EPA CLP } \\
\text { EPA CLP } \\
\text { EPA CLP } \\
\text { EPA CLP } \\
\text { EPA CLP } \\
\text { EPA CLP } \\
\text { EPA CLP } \\
\text { EPA CLP } \\
\text { EPA CLP } \\
\text { EPA CLP } \\
\text { EPA CLP } \\
\text { EPA CLP } \\
\text { EPA } 8260 \\
\text { EPA } 8260\end{array}$ \\
\hline
\end{tabular}




\begin{tabular}{cccccccc}
\hline USSRAP_ID & DATE_SAM & & PARAMETER & CONC & DL & UNITS & METHOD \\
\hline SP-6303-0498-L & $04 / 20 / 98$ & TRICHLOROETHENE (TCE) & ND & 1.0 & UG/L & EPA 8260 \\
SP-6303-0198-L & $01 / 15 / 98$ & VINYL CHLORIDE & ND & 10 & UG $/ L$ & EPA CLP \\
SP-6303-0298-L- & $02 / 25 / 98$ & VINYL CHLORIDE & ND & 10 & UG/L & EPA CLP \\
SP-6303-0398-L & $03 / 16 / 98$ & VINYL CHLORIDE & ND & 10 & UG/L & EPA CLP \\
SP-6303-0498-L & $04 / 20 / 98$ & VINYL CHLORIDE & ND & 10.0 & UG/L & EPA CLP \\
SP-6303-0198-L & $01 / 15 / 98$ & XYLENES, TOTAL & ND & 10 & UG/L & EPA CLP \\
SP-6303-0298-L- & $02 / 25 / 98$ & XYLENES, TOTAL & ND & 10 & UG/L & EPA CLP \\
SP-6303-0398-L & $03 / 16 / 98$ & XYLENES, TOTAL & ND & 10 & UG/L & EPA CLP \\
SP-6303-0498-L & $04 / 20 / 98$ & XYLENES, TOTAL & ND & 10.0 & UG/L & EPA CLP \\
\hline
\end{tabular}





\section{Second Quarter 1998 Air Particulate Results}

LOCATION ID

$$
\text { QUARTERLY AVERAGE GROSS }
$$

ALPHA CONCENTRATION (uCi/ml)

$1.27 \mathrm{E}-15$
$1.71 \mathrm{E}-15$
$1.45 \mathrm{E}-15$
$1.69 \mathrm{E}-15$
$1.46 \mathrm{E}-15$
$1.73 \mathrm{E}-15$
$1.12 \mathrm{E}-15$
$1.32 \mathrm{E}-15$
$1.23 \mathrm{E}-15$
$1.35 \mathrm{E}-15$
$1.18 \mathrm{E}-15$
$9.62 \mathrm{E}-16$
$1.04 \mathrm{E}-15$
$1.29 \mathrm{E}-15$
$1.14 \mathrm{E}-15$
$1.30 \mathrm{E}-15$
STANDARD

DEVIATION

$5.76 \mathrm{E}-16$

$1.01 \mathrm{E}-15$

5.87E-16

8.39E-16

$7.16 \mathrm{E}-16$

$7.46 \mathrm{E}-16$

$3.51 \mathrm{E}-16$

3.70E-16

6.02E-16

$6.15 \mathrm{E}-16$

$2.78 \mathrm{E}-16$

$6.26 \mathrm{E}-16$

$5.99 \mathrm{E}-16$

4.19E-16

$3.98 \mathrm{E}-16$

$5.86 \mathrm{E}-16$
NO. WEEKS

COLLECTED

13

13

13

13

13

13

11

13

13

13

13

13

13

13

13

13

* Background monitoring location 


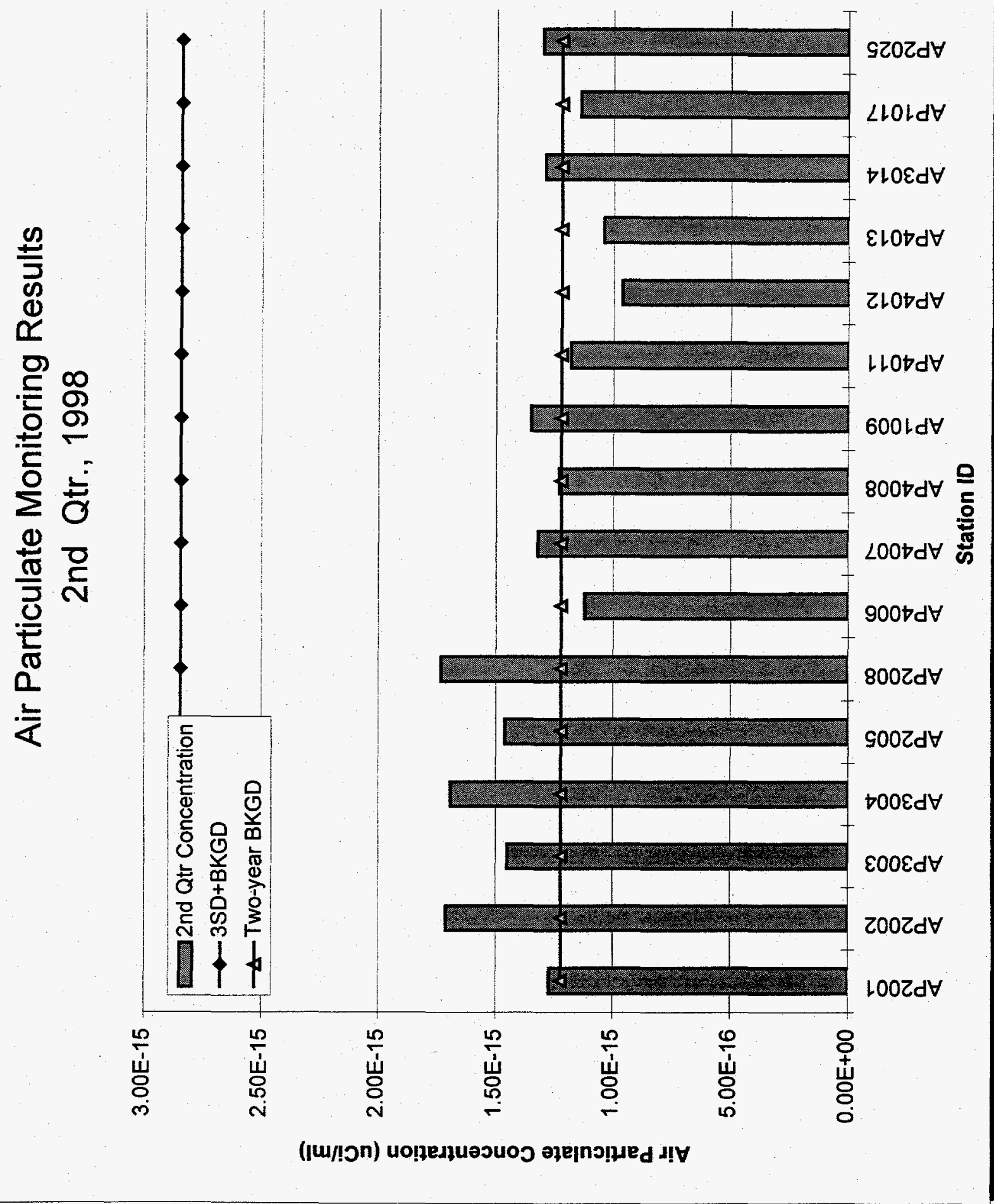




\section{First Quarter 1998 Alpha Track Radon Monitoring Results}

LOCATION ID

AVERAGE (pCi/L)

PERCENT OF DCG(a)

Weldon Spring Quarry

RD-1002

0.3

3

RD-1005

0.3

3

Weldon Spring Chemical Plant

RD-2002

0.1

0

$\mathrm{RD}-2004$

0.2

0

RD-2006

0.2

0

RD-2007

0.3

3

RD-2025.

0.1

0

Weldon Spring Raffinate Pits

RD-3001

0.2

0

RD-3002

0.3

3

RD-3003

0.4

7

RD-3004

0.2

0

RD-3005

0.2

RD-3007

$--$

RD-3008

0.2

0

RD-3009

0.3

N/A

RD-3010

0.5

0

RD-3011

0.3

RD-3012

0.6

10

RD-3013

0.9

13

RD-3014

0.5

23

RD-3015

0.2

10

0

\section{Off Site}

RD-4001

0.2

0

RD-4002

0.1

0

RD-4003

0.1

RD-4005(b)

0.2

RD-4007

0.2

RD-4009(b)

0.2

RD-4013

0.1

Combined 52-week Background

0.2

0

N/A

0

N/A

0

N/A

(a) Derived Concentration Guide. Percent of the DCG is determined by subtracting the combined 52-week background average from a station's reported quarterly concentration, dividing by $3 \mathrm{pCi} / \mathrm{L}$ (the $\mathrm{DCG}$ for radon), and multiplying by 100 .

(b) Denotes background monitoring location.

- Missing detector. 
Integrated Radon Concentration (pCi/L)

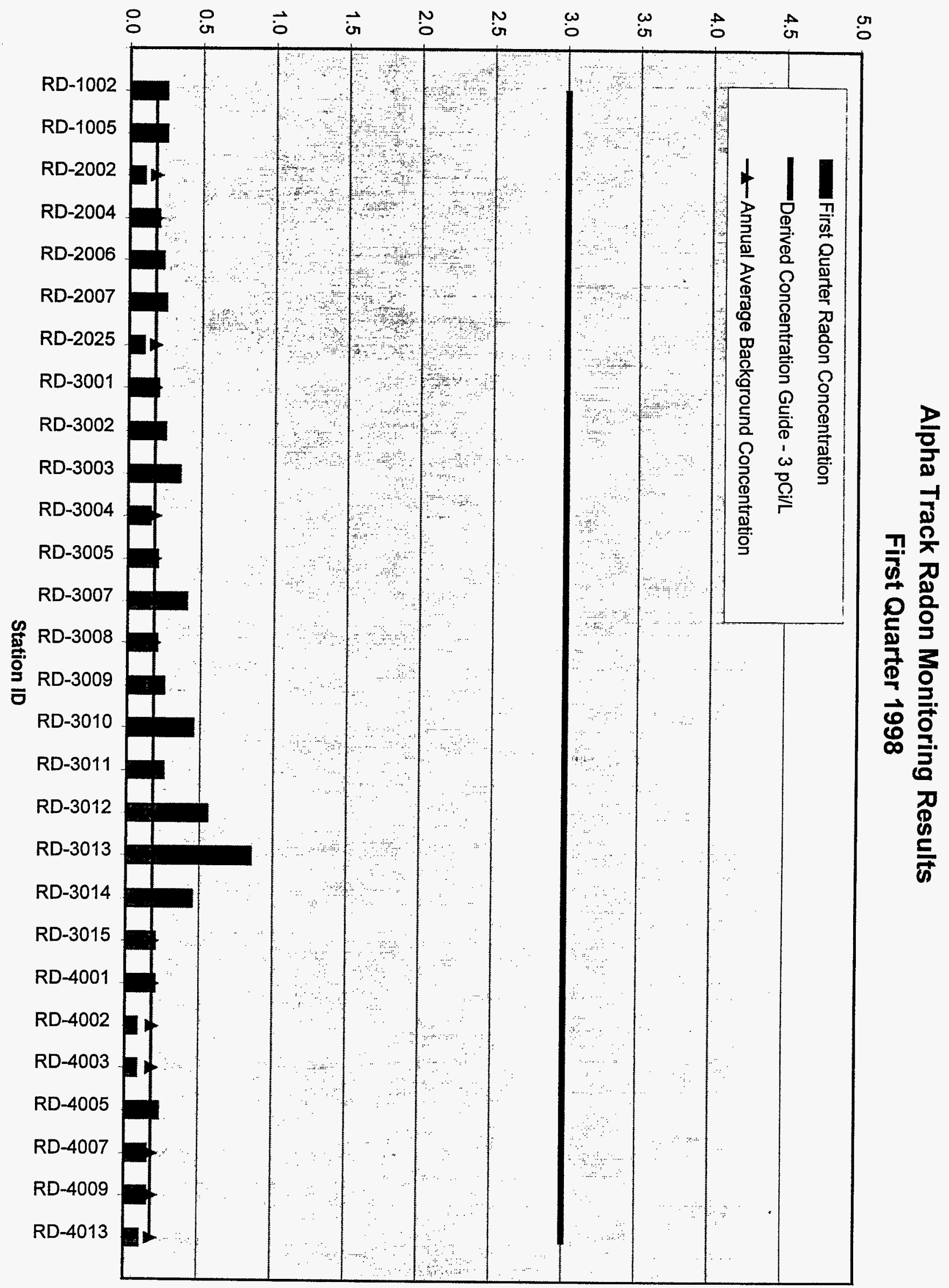


First Quarter 1998 Alpha Track Thoron Monitoring Results

LOCATION ID

AVERAGE $(\mathrm{pCi} / \mathrm{L}) \quad$ PERCENT OF DCG $^{(\mathrm{a})}$

Weldon Spring Quarry

RD-1002

0.0

0

Weldon Spring Chemical Plant

RD-2002

0.0

0

RD-2004

0.0

0

RD-2006

0.0

0

RD-2007

0.1

0

RD-2025

0.0

0

Weldon Spring Raffinate Pits

RD-3001

0.0

0

RD-3002

0.0

0

RD-3003

0.3

7

RD-3004

0.0

RD-3007

$-$

RD-3010

0.3

0

RD-3014

0.1

N/A

7

0

Off Site

RD-4001

0.0

0

RD-4002

0.0

0

RD-4003

0.0

0

$\mathrm{RD}-4005^{(\mathrm{b})}$

0.0

RD-4007

0.0

N/A

RD-4009 ${ }^{(b)}$

0.0

RD-4013

0.0

Combined 52-Week Background

0.1

0

N/A

0

N/A

(a) Derived Concentration Guide. Percent of the DCG is determined by subtracting the combined 52-week background average from a station's reported quarterly concentration, dividing by $3 \mathrm{pCi} / \mathrm{L}$ (the $\mathrm{DCG}$ for thoron), and multiplying by 100 .

(b) Denotes background monitoring location.

-- Missing alpha track detector. 


\section{Alpha Track Thoron Monitoring Results}

\section{First Quarter 1998}

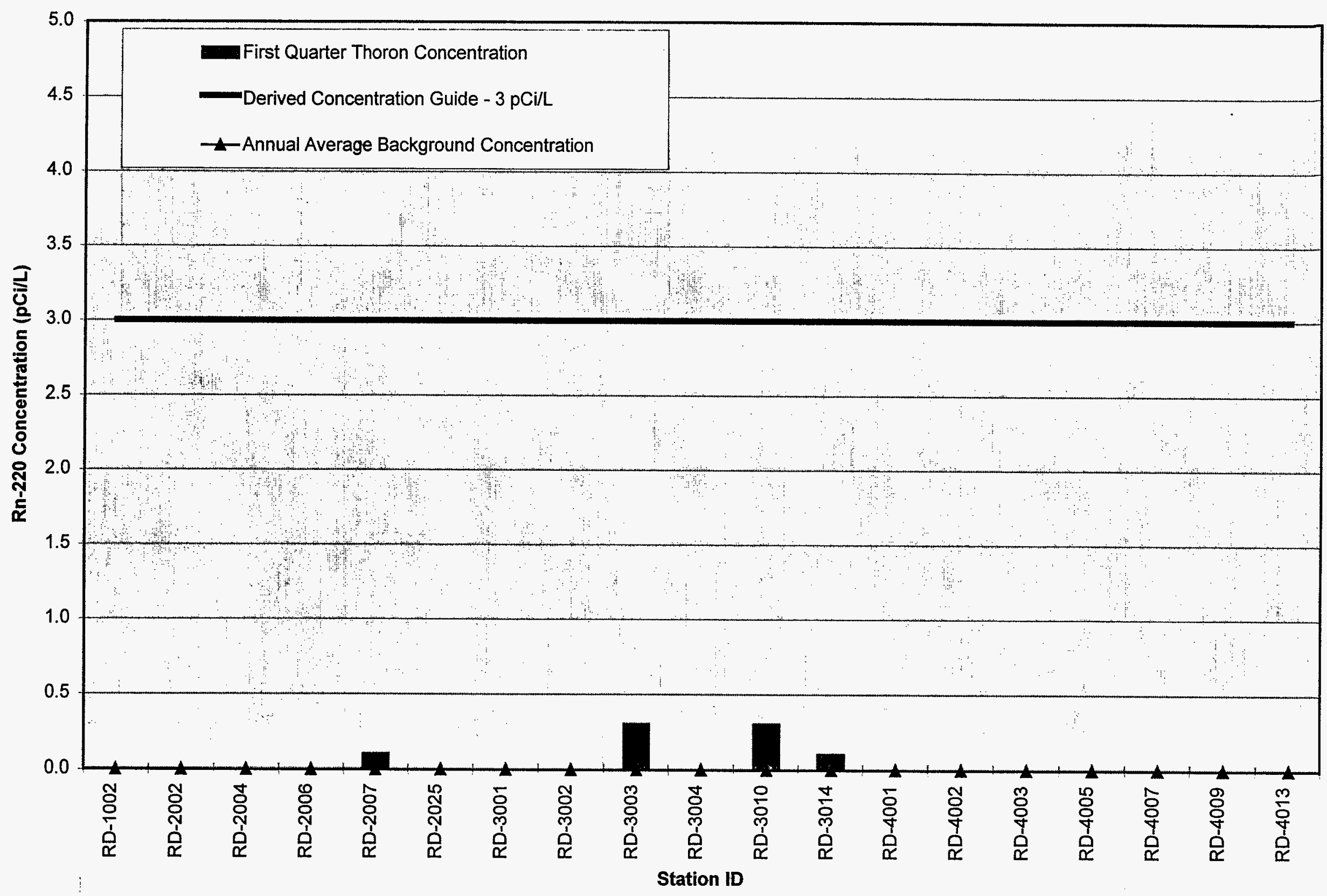




\section{FIRST QUARTER 1998 ENVIRONMENTAL TLD RESULTS}

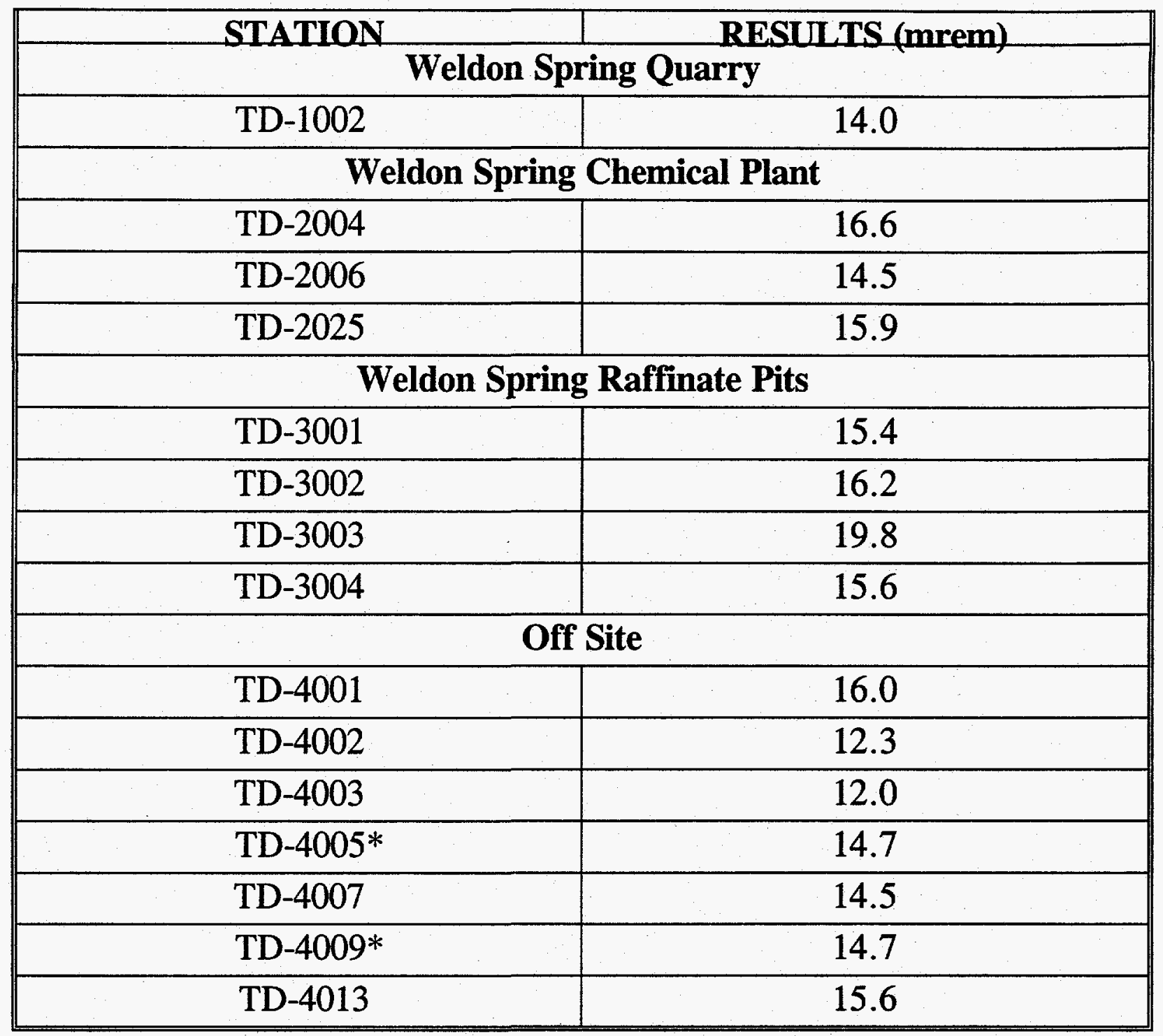

* Background monitoring station 


\section{Environmental TLD Monitoring Results}

First Quarter 1998

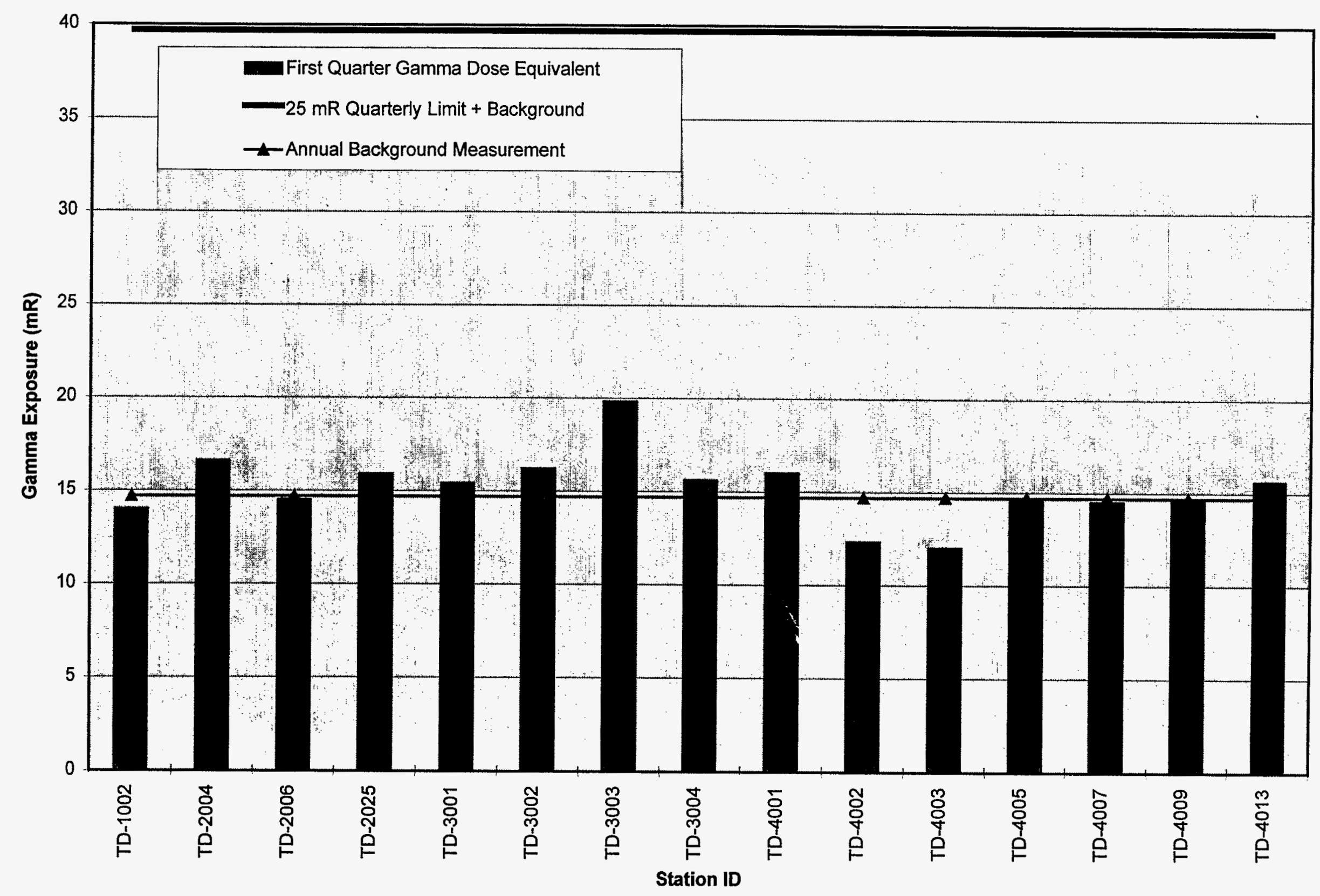

